\title{
TRANSFERABLE LOCALIZED ORBITALS FOR ACYCLIC HYDROCARBONS
}

\author{
Ph.D. Thes is Submitted to lowa State University, \\ February, 1973
}

W. B. England

\author{
Ames Laboratory, USAEC \\ lowa State University
}

Ames, lowa 50010

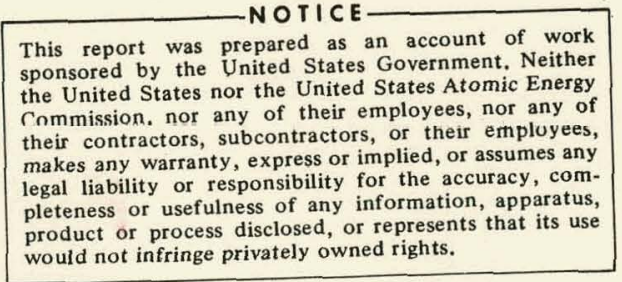

Date of Manuscript: February, 1973

PREPARED FOR THE U. S. ATOMIC ENERGY COMMISSION DIVISION OF RESEARCH UNDER CONTRACT NO. W-7405-eng-82 


\section{DISCLAIMER}

This report was prepared as an account of work sponsored by an agency of the United States Government. Neither the United States Government nor any agency Thereof, nor any of their employees, makes any warranty, express or implied, or assumes any legal liability or responsibility for the accuracy, completeness, or usefulness of any information, apparatus, product, or process disclosed, or represents that its use would not infringe privately owned rights. Reference herein to any specific commercial product, process, or service by trade name, trademark, manufacturer, or otherwise does not necessarily constitute or imply its endorsement, recommendation, or favoring by the United States Government or any agency thereof. The views and opinions of authors expressed herein do not necessarily state or reflect those of the United States Government or any agency thereof. 


\section{DISCLAIMER}

Portions of this document may be illegible in electronic image products. Images are produced from the best available original document. 


This report was prepared as an account of work
sponsored by the United States Government. Neither
the United States nor the United States Atomic
Energy Commission, nor any of their employees, nor
any of their contractors, subcontractors, or their
employees, makes any warranty, express or implied,
or assumes any legal liability or responsibility for the
accuracy, completeness or usefulness of any
information, apparatus, product or process disclosed,
or represents that its use would not infringe privately
owned rights.

Available from: National Technical Information Service Department $A$

Springfield, VA 22151

Price: Microfiche $\$ 0.95$ 
Transferable localized orbitals for acyclic hydrocarbons

$$
\text { by }
$$

Walter Bernard England

\author{
A Dissertation Submitted to the \\ Graduate Faculty in Partial Fulfillment of \\ The Requirements for the Degree of \\ DOCTOR OF PHILOSOPHY
}

Department: . Chemistry

Major: Physical Chemistry

Approved:

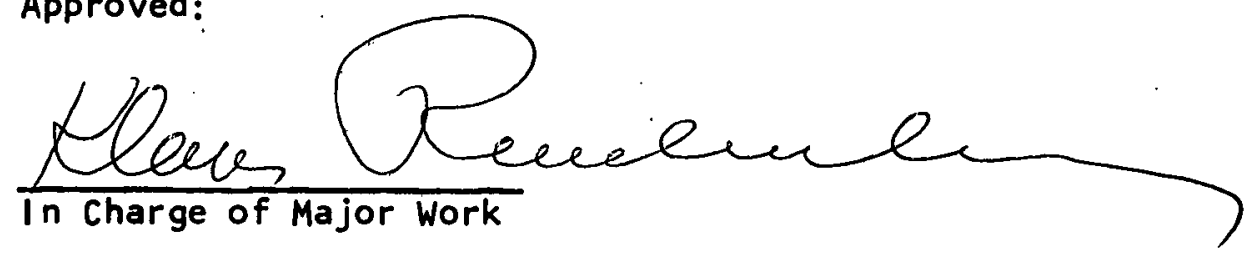

Goh Der the Major Department.

\title{
Chand.tt EPodemck
}

For the Graduate College

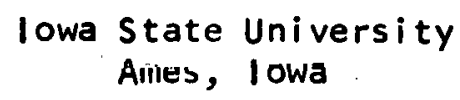


TABLE OF CONTENTS

ABSTRACT

INTRODUCTION

Page

xv

MOLECULAR ORBITAL THEORY

Introduction

CNDO and INDO

GEOMETRY AND HYBRIDIZATION

Introduction

Geometries and Numberings of Atoms and Hybrid

Bas is Functions

Pair Equivalent Hybrids in Mutualíy Perpendicular

Planes

Ethynyl Carbon (Single Bond and Triple Bond) 60

\section{ENERGY LOCALIZED MO'S}

Introduction

Localization Equations

Semiempirical Energy Localization

Starting Orbitals

First Order Localization

LMO's as Two-Center Orbitals Perturbed by Bond-Bond Interactions

Energy Localized Orbitals from PseudoEigenvalue Equations

The LCAO Expansions

MOLECULE INVARIANT LHO FRAGMENTS

Local Delocalization and LMO Fragments

LMO Characteristics of Fragments

Characterization of LMO Fragments by Bond Regions 
MI ORBITAL BOND FRAGMENTS

General Properties $\quad 77$

$\begin{array}{ll}\text { Alkyl } \mathrm{CH} \text { Bonds } & 78\end{array}$

Alkyl CC Bonds $\quad \therefore \quad 87$

Primary Vinyl CH Bonds $\quad 94$

Secondary Vinyl CH Bonds $\quad \cdot \quad 102$

$\begin{array}{ll}\text { Vinyl CC Single Bonds } & 105\end{array}$

CC Double Bonds $\quad 105$

$\begin{array}{ll}\text { Ethynyl CH Bonds } & 108\end{array}$

Ethynyl CC Single Bonds 113

CC Triple Bonds : 113

MI ORBITAL GEMINAL FRAGMENTS $\quad: 11$

$\begin{array}{ll}\text { Introduction } & 117\end{array}$

$\begin{array}{lr}\text { Alkyl CH Fragments } & 118\end{array}$

$\begin{array}{ll}\text { Alkyl CC Fragments } & 128\end{array}$

$\begin{array}{lr}\text { Primary Vinyl CH Fragments } & 133\end{array}$

Secondary Vinyl CH Fragments $\quad 134$

Single Bond Vinyl CC Fragments $\quad 135$

Double Bond Fragments $\quad 137$

$\begin{array}{ll}\text { Ethynyl CH Fragments } & 138\end{array}$

Single Bond Ethynyl CC Fragments $\quad 139$

Triple Bnnd Fragments 139 


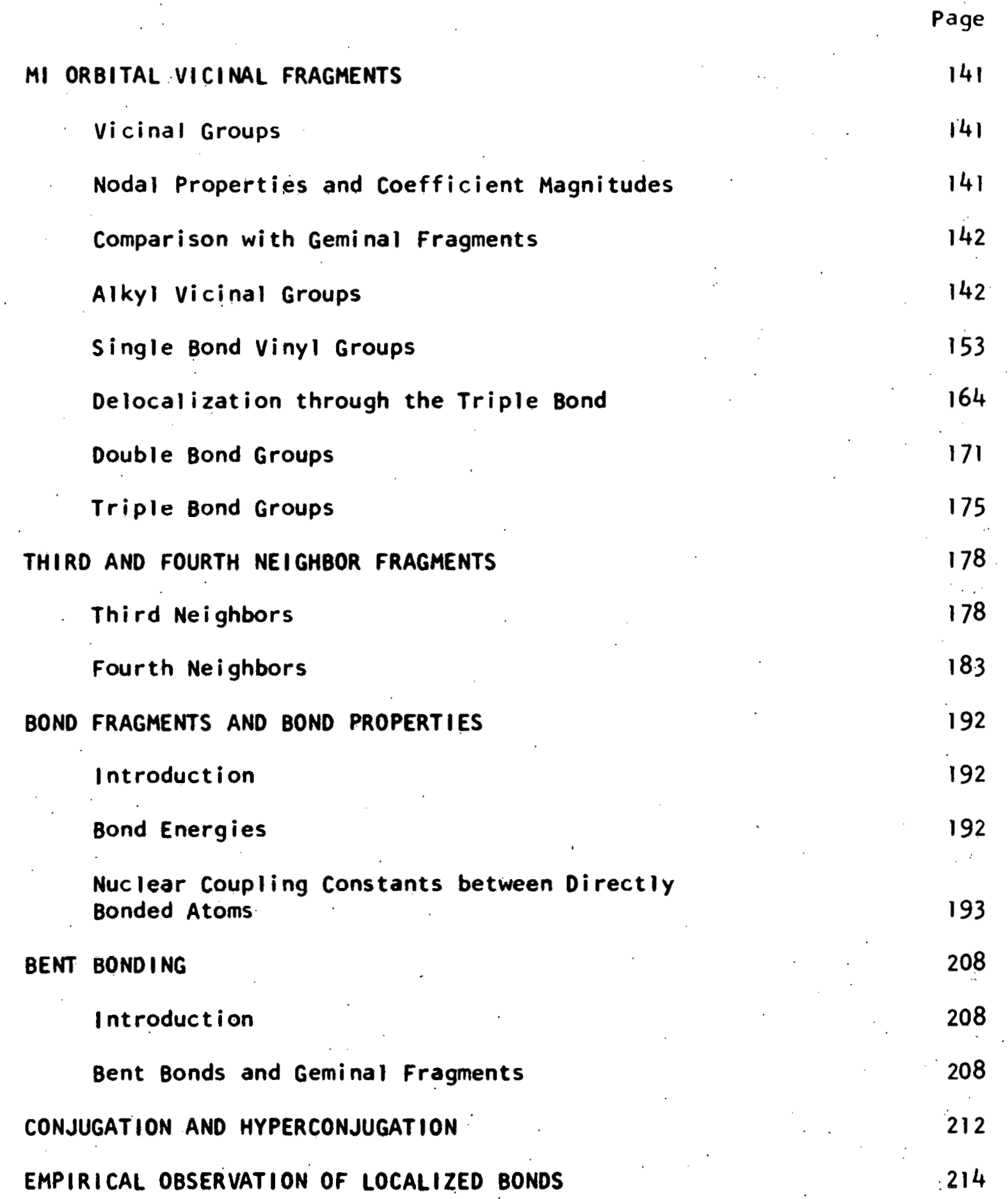


CANONICAL AND VIRTUAL ORBITALS

Introduction

Virtual Orbitals

Canonical Orbitals

218

MODEL MOLECULES

220

APPENDIX: INTERPRETATIONS OF LMO'S

221

LITERATURE CITED 
viii

\section{LIST OF TABLES}

Page

Table 1. Geometry and LCAO-MO's for propane 24

Table 2. Geometry and LCAO-MO's for eclipsed ethane 25

Table 3. Geometry and LCAO-MO's for butane $\quad 26$

Table 4. Geometry and LCAO-MO's for butadiyne 27

Table 5. Geometry and LCAO-MO's for 2-butyne 28

Table 6. Geometry and LCAO-MO's for 1,4-pentadiyne 29

Table 7. Geometry and LCAO-MO's for methane 30

Table 8. Geometry and LCAO-MO's for acetylene 31

Table 9. Geometry and LCAO-MO's for s-gauche-l-butene 32

Table 10. Geometry and LCAO-MO's for s-cis-, s-trans-1, 4pentadi ene

Table 11.: Geometry and LCAO-MO's for pentene 34

Table 12. Geometry and LCAO-MO's for vinyl ethynyl ethane 35

Table 13. Geometry and LCAO-MO's for vinyl ethynyl methane 36

Table 14. Geometry and LCAO-MO's for isopropyl acetylene 37

Table 15. Geometry and LCAO-MO's for ethane 38

Table 16. Geometry and LCAO-MO's for pentane 39

Table 17. Geometry and LCAO-MO's for isobutane 40

Table 18. Geometry and LCAO-MO's for isopentane 41

Table 19. Geometry and LCAO-MO's for s-gauche-isopropyi
ethylene

Table 20. Geometry and LCAO-MO's for ethylono 43

Table 21. Geometry and LCAO-MO's for propene 44

Table 22. Geometry and LCAO-MO's for s-cis-propene 45

Table 23. Geometry and LCAO-MO's for butene 46 
Table 24. Geometry and LCAO-MO's for s-cis-butene 47

Table 25. Geometry and LCAO-MO's for 2-butene 48

Table 26. Geometry and LCAO-MO's for isobutene 49

Table 27. Geometry and LCAO-MO's for butadiene 50

Table 28. Geometry and LCAO-MO's for propyne 51

Table 29. Geometry and LCAO-MO's for butyne 52

Table 30. Geometry and LCAO-MO's for pentyne 53

Table 31. Geometry and LCAO-MO's for vinyl acetylene 54

Table 32. Geometry and LCAO-MO's for isoprene 55

Table 33. Geometry and LCAO-MO's for s-trans-isopropyl
ethylene

Table 34. Angles in degrees and bondlengths in. Angstroms
assigned by the model-builder to acyclic paraffins

Table 35. Comparison of localized, least mean square and perturbation MO's in ethane 66

Table 36. Bond and geminal fragments in methane and primary CH LMO's 79

Table 37. Bond and geminal fragments in secondary $\mathrm{CH}$ LMO's 80

Table 38. Bond and geminal fragments in tertiary $\mathrm{CH}$ LMO's 81

Table 39. Bond and geminal fragments in cis primary CH LMO's 82

Tabile 40. Substituent effects on $\mathrm{CH}$ bond fragments 86

Table 41. Bond and geminal fragments in saturated primary CC LMO's

Table 42. Bond and geminal fragments in unsaturated primary CC LMO's

Table 43. Bond and geminal fragments in saturated secondary CC LMO's. 
Table 44. Bond and geminal fragments in unsaturated secondary CC LMO's

Table 45. Bond and geminal fragments in tertiary CC LMO's

Table 46. Substituent effects on bond fragments in $C C$ and double banana bonds

Table 47. Bond and geminal fragments in ethylene and primary vinyl CH LMO's cis to the substituent

Table 48. Bond and geminal fragments in ethylene and primary vinyl CH LMO's trans to the substituent

Table 49. Bond and geminal fragments in ethylene and primary vinyl CH LMO's in disubstituted systems

Table 50. Bond and geminal fragments in secondary vinyl $\mathrm{CH}$ LMO's

Table 51. Bond and geminal fragments in vinyl CC (alkyl) LMO's 106

Table 52. Bond and geminal fragments in vinyl CC (vinyl or ethynyl) LMO's

Table 53. Bond and geminal fragments on primary carbons in double banana LMO's

Table 54. Bond and geminal fragments on primary and tertiary carbons in double banana LMO's

110

Table 55. Bond and geminal fragments on secondary carbons in banana LMO's

Table 56. Bond and geminal fragments on ethynyl carbons in $\mathrm{CH}$ and CC LMO's

Table 57. Bond and geminal fragments on primary ethynyl carbons in banana LMO's

Table 58. Bond and geminal fragments on secondary ethynyl carbons in banana LMO's

Table 59. Prototype geminal CH fragments on primary carbons

Table 60. Prototype geminal CH fragments on secondary carbons

Table 61. Prototype geminal CH fragments on tertlary carbons 
Table 62. Substituent effects in geminal $\mathrm{CH}$ fragments with the introduction of a $\mathrm{CC}$ bond

Table 63. Substituent effects in geminal $\mathrm{CH}$ fragments with the introduction of a double bond

Table 64. Substituent effects in geminal $\mathrm{CH}$ fragments with the introduction of a triple bond

Table 65. Substituent effects in geminal $\mathrm{CH}$ fragments with the introduction of double and triple bonds 125

Table 66. Substituent effects in cis geminal $\mathrm{CH}$ fragments

Table 67. Prototype geminal CC fragments on primary and secondary carbons

Table 68. Prototype tertiary geminal CC fragments

Table 69. Substituent effects in alkyl geminal CC fragments with the introduction of CC bonds

Table 70. Substituent effects in alkyl geminal. CC fragments with the introduction of banana bonds

Table 71. Overflow onto primary $\mathrm{CH}$ fragments in $\mathrm{CH}$ LMO's 144

Table 72. Overflow onto secondary $\mathrm{CH}$ fragments in $\mathrm{CH}$ LMO's

Table 73. Overflow onto secondary $\mathrm{CH}$ fragments in alkyl $\mathrm{CH}$ LMO's

Table 74. Overflow onto tertiary $\mathrm{CH}$ fragments in alkyl $\mathrm{CH}$ LMO's

Table 75. Overflow onto primary and secondary $\mathrm{CH}$ fragments in CC LMO's

Table 76. Overflow onto primary $\mathrm{CH}$ fragments in double banana LMO's

Table 77. Overflow onto secondary and tertiary $\mathrm{CH}$ fragments from banana LMO's

Table 78. Overflow onto CH fragments in triple banana LMO's

Table 79. Overflow onto secondary alkyl CC fragments in CH LMO's 
Table 80. Overflow onto tertiary alkyl CC fragments in CH LMO's

Table 81. Overflow onto unsaturated secondary alkyl CC fragments in $\mathrm{CH}$ LMO's

Table 82. Overfiow onto unsaturated tertiary alkyl CC fragments in CH LMO's

Table 83. Overflow onto secondary alkyl CC fragments in CC LMO'S

Table 84. Overflow onto aklyl CC fragments in double banana LMO's

Table 85. Overflow onto alkyl CC fragments in triple banana LMO's

Table 86. Overflow thru the double bond onto primary vinyl CH fragments

Table 87. Overflow through the double bond onto secondary $\mathrm{CH}$ fragments in primary and secondary vinyl CH LMO's

Table 88. Overflow through the double bond onto vinyl unsaturated CC fragments

Table 89. Overflow through the double bond onto vinyl CC fragments in primary vinyl CH LMO's

Table 90. Overflow onto secondary vinyl $\mathrm{CH}$ fragments in $\mathrm{CH}$ LMO's

Table 91. Overflow onto secondary vinyl CH fragments in CC LMO's

Table 92. Overflow onto tertiary vinyl CC fragments in CH LMO's 169

Table 93. Overflow thru the triple bond 170

Table 94. Overf low onto double banana fragments in CH LMO's 172

Table 95. Overflow onto secondary-secondary and tertiary double banana: fragments in $\mathrm{CH}$ LMO's

Table 96. Overflow onto double banana fragments in CC and banana LMO's 
Table 97. Overflow onto triple banana fragments in CH LMO's

Table 98. Overflow onto triple banana fragments in $\mathrm{CC}$ and banana LMO's

Table 99. T overflow onto third neighbor fragments

Table 100. C overflow onto third neighbor fragments

Table 101. S overflow onto third neighbor fragments

Table 102. $S^{\prime}, L$ and $P$ overflow onto third neighbor fragments

Table 103. TT, TC and TP overflow onto fourth neighbor fragments

Table 104. CT, CC and CP overflow onto fourth neighbor fragments 190

Table 105..PL, LP and LL overflow onto fourth neighbor fragments

Table 106. Comparison of alkyl bond atomization energies and bond fragment energies in kilocalories per mole

Table 107. Comparison of vinyl bond atomization energies and bond fragment energies in kilocalories per mole

Table 108. Comparison of ethynyl bond atomization energies with bond fragment energies in ki localories per mole

Table 109. Average energies and coupling constants in ethane, ethylene and acetylene

Table 110. Comparison of experimental and calculated $\mathrm{CH}$ coupling constants

Table IIl. Calculated alkyl coupling constants

Table 112. Calculated vinyl and ethynyl coupling constants

Table 113.: Vinyl and ethynyl coupling constants in some conjugated systems

Table 114. Orbital deviations from the bond skeleton in degrees

Table 115. Primary $\mathrm{CH}$ orbital deviations from the bond skeleton in degrees 
xiv

\section{LIST OF FIGURES}

Page

Figure 1. Geometries and numberings of atoms and hybrid basis functions

Figure 2. Bond skeleton and relative orientation nomenclatures

Figure 3. Bond skeletons for primary vinyl $\mathrm{CH}$ fragments cis to the substituent

Figure 4. Bond skeletons for primary vinyl $\mathrm{CH}$ fragments trans to the substituent

Figure 5. Bond skeletons for third neighbor overflow

Figure 6. Bond skeletons for fourth neighbor overflow 
Transferable localized orbitals for acyclic hydrocarbons

Wialter Bernard England

Under the supervision of Klaus Ruedenberg

From the Department of Chemistry

lowa State University

INDO-SCF localized molecular orbital expansion coefficients in

Slater and hybrid atomic orbital bases are given for thirty-three acyclic hydrocarbons. The localized orbitals are found to extend slightly and regularly into regions away from the bond itself. Hybrid atomic orbital expansion coefficients are given for molecule invariant (MI) fragments, the somcalled bond fragments, geminal fragiments, vicinal fragments, and third and fourth neighbor fragments. The first three classes are discussed in detail. With these fragment coefficients and the model geometries employed, iacalized orbitals for acyclic hydrocarbon molecules can be assembled without solving equations.

Applications of the Ml bond fragment coefficients to bond energies and nuclear spin coupling constants between directly bonded atoms are made. Bent tonding and the notion of geminal delocalization are shown to be intimately related. The relative behavior of bond and vicinal fragment coefficients is connected to conjugation and hyperconjugation.

several novei results regarding energy localization are also presented. These include simple methods for obtaining close approximations

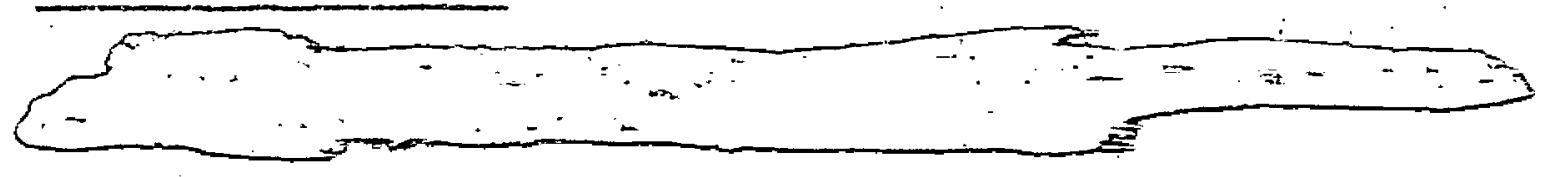


to energy localized molecular orbitals and an accompanying convergent first order localization algorithm. Similar applications of a suggested pseudo-eigenvalue method and its accompanying self-consistent-field iteration process are found to not converge. 


\section{NTRODUCTION}

Some of man's most powerful learning tools are his ability to organize, compare and draw upon experience. With them, he understands by understanding relatively, by first understanding what is easily understood, and is able to use today what he learned, with whatever means, yesterday.

This thesis combines two powerful techniques: rigorous molecular quantum mechanics and classical valence theory. The former provides a firm theoretical basis for investigation and counteracts one's own prejudices and inconsistencies, while the latter furnishes the concepts, suggestiveness and subjective intuition so successfully employed even in the years before quantum mechanics $(1-4)$, and certainly before very complete theoretical investigations were available.

The combination is performed with the energy. localized representation (5-8) of closed-shell INDO (9-13) molecular orbital theory (14). It like all others - is a subjective representation, as has been known for many years (15), but is nonetheless rigorous and has proved exceedingly useful for studying phenomena which don't involve electronic excitations $(6,7,16-25)$. The applications at present will be to the electron distributions in acyclic hydrocarbons, with emphasis on the transferability and inherent delocalization of the localized orbitals which describe the electronic motion. By using INDO, it overcomes the economic and technical problems (computer storage and time, multicenter integrals, etc.) encountered in ab initio methods, which would restrict the study to just a few molecules $(7,16,17,24,25)$, at the expense of having a more phenomenological description. However, experience has shown this to create a problem 
only insofar as absolute energies are concerned, which are not at issue here. In fact, recent ab initio results $(25-28)$ indicate that trends in INDO and minimal basis theory differ from those of near Hartree-Fock results in similar ways (29).

Unlike some studies which concern themselves with the examination of properties $(16,17,20,24)$, the present philosophy is that wave functions are more fundamental, and therefore more useful and interesting than any of the expectation or pseudo-expectation (22) values they provide. Also unlike some other studies, it does not consider localized orbitals perfectly confined to one or two centers $(30-33)$, and examines transferabllity in much greater detail $(6,7,16,17,24)$. The former obtains because of the rigorous approach (5) used, and differs from others (30-33) which are closer to classical valence theory, while the latter makes transferability as quantitative as is reasonably possible, illustrating its use and theoretical meaningfulness, confirming what has long been appreciated (or suspected) by chemists $(34-38)$.

Dirac's bra and ket notation (39) will be used throughout. Since closed-shell molecular orbital theory is well documented (14) and appears in many texts $(34,40-46)$ it will not be discussed in very great detail in this work. The INDO approximations (9-13) will be presented descriptively, with attention to the basic reasoning, which leads to some interesting features.

Very little discussion will be given the model geometries employed (47), which are closely related to the hybrid atomic orbitals defined on each atom. The former were obtained with the 'Model-Builder' program (47) 
automatically, thereby rendering an unpleasant technical problem trivial. The latter serve as a consistent set of atomic basis functions which form perfectly localized first approximations to the localized orbitals, and hence display their behavior very effectively.

Energy localization is also well documented $(5,6,8)$ and beginning to find its way into quantum chemistry books $(45,48)$. The important features and needed equations will be given, as will some new contributions of the present work to the computation of the localized orbitals.

The major effort will be the analysis of the localized orbitals for the thirty-three molecules studied. The orbitals will be partitioned into fragments that are independent of the molecules, and each group will be classified and discussed separately, revealing their behavior and the ways in which each transfers. Following this, discussions will be given of some bond properties and important chemical topics, including the direct observation and physical significance of transferrable localized bonding. Methods for obtaining the unoccupied and canonical symmetry molecular orbitals wi Il also be given.

Finally, the programs which made this project possible are the works of many hands. The energy localization methods were built from the originals of Professor Clyde Edmiston (Wyoming University). The INDO selfconsistent-field and integrals packages originated in Professor John Pople's research group at Carnegie-Mellon University, and the 'Model-Builder' was written at the same place by Professor Mark Gordon of North Dakota State University. Together, these are around 20,000 cards, or about ten IBM boxes! 


\section{MOLECULAR ORBITAL THEORY}

\section{introduction}

Molecular orbital (MO) theory was introduced more than forty years ago by Hund $(49-51)$ and Mulliken $(52,53)$, and now forms a major portion of every modern theoretical chemistry text and plays an active role in chemical, physical and biological research of all kinds (54). There are perhaps two developments which led to this state of affairs more than any others:

1. The introduction of the Mo method to organic chemistry by Hücke I (55).

2. The appearance of the Roothaan Equations (14) and the rise of computers.

The former stimulated virtually all of recent pi electron organic chemistry, while the latter's rigorous reduction of the Mo problem to iterative solution of matrix equations fits perfectly with machine calculation. Along with the less appreciated free-electron MO theory $(38,56-58)$ it is thus fair to say that these contributed greatly, and will continue to do so, each in their way, to the mathematical tractability of Mo theory and its ability to reproduce, predict and interpret experimental results.

\section{CNDO and INDO}

These are all-valence-electron linear-combination-of-atomic-orbitals (LCAO)-MO self-consistent-field (SCF) theories developed by Pople and coworkers (9-13) in which the approaches and approximations used so fruitfully in pi electron theory $(41,59)$ are suitably introduced into the Roothaan 
Equations. The most novel and far-reaching feature is their use of unitary invariance, i.e. the Roothaan Equations are invariant both to unitary transformations among the occupied MO's (14) and the basis functions (9). The former obtains because any single determinant wave function is so invariant (15), and has lately been widely appreciated because of the interest in energy localization. The latter was the one used by Pople et al. to guide the development of their approximate LCAO-MO-SCF theories.

The discussion was first restricted to atomic bases, eliminating from consideration all unitary transformations that mix atomic orbitals ( $A 0^{\prime} \mathrm{S}$ ) on one center with those on another. Subsequent approximations were then required to be invariant to the remaining possibilities: rotations of the local coordinate axes; and hybridization changes. To this was added the 'neglect of differential overlap'

$$
f(\underline{x}) \cdot g(\underline{x}) d V=0 \text {, if } f \neq g
$$

where $f$ and $g$ are $A O^{\prime} s$, thus leading to the formulation of CNDO:

1. The Slater AO basis $(9,10,59)$ is assumed orthogonal (thus neither admits a truly extensional basis, only a means of approximating expectation values).

2. All integrals are reduced to one- or two-centers (hence the total energy reduces to a sum of one- and two-atom terms $(9,60)$.

3. One-center, one-electron matrix elements are obtained empirically by equating the average of the $A O$ ionization potentials and electron affinities to the theoretical energy of the valence electron in the corresponding atomic state. 
4. After some experience (11) with the earliest versions $(9,10)$, the two-center, one-electron Coulomb integrals (the analogous integrals which involve two different $\mathrm{AO}^{\prime}$ 's on the same center are zero because of Eq. I) were assigned values which neglect 'penetration' effects (those leading to attraction even when the bond order between the two atoms is zero, i.e. electrons in an orbital on one atom 'penetrate' the shell of another).

5. Two-center, one-electron resonance integrals were parameterized to give the best overall fit with minimal basis ab initio LCAO-SCF-MO diatomic molecule calculations. (strictly, this violates the neglect of differential overlap, but these integrals are the one-electron interference density contributions (19) and hence must be included in any theory hoping to describe chemical bonds $(61))$.

6. All two-electron integrals are reduced to one- or two-center Coulomb integrals over the valence s functions.

Notice that - because of 3 and 5 - valence $A O^{\prime}$ 's are distinguished only in the one-electron integrals. INDO (12) also distinguishes them in the two-electron integrals by retaining all one-center, two-electron. coulomb and exchange integrals (which affects the one-electron matrix elements via 3), and in keeping with the guidelines, they are given values quoted by slater $(62)$ as representing the best fit with atomic experimental data. Again this violates the neglect of differential overlap, but the absence of spherical averaging is crucial for energy localization (63). The differences mentioned in the last paragraph are the only ones separating CNDO from INDO. There are, however, some interesting features that follow from 1 which have not been mentioned yet: the very simple and 
unambiguous definitions of the atomic quasiclassical $(19,61)$ densities

$$
Q(x, A)=\sum_{L i} \sum_{i}^{A}\left(C_{L i} X_{i}(\underline{x})\right)^{2}
$$

and diatomic interference $(19,61)$ densities

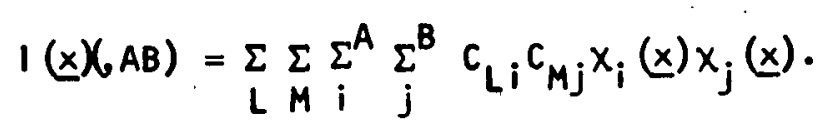

The sums on $i$ and $j$ are over all AO's $X$ on atoms $A$ and $B$, respectively, while $L$ and $M$ are sums over the occupied MO's u

$$
u_{L}(x)=\sum_{A} \sum_{i} c_{L i} x_{i}(\underline{x})
$$

with expansion coefficients $C_{L i}$. The former yields the usual definition of Mulliken's gross atomic populations $(64,65)$, likewise not troubled with overlap partitioning

$$
Q(A)=\langle Q(\underline{x}, A)\rangle,
$$

and both satisfy the conservation equations. (61)

$$
\begin{aligned}
& \sum Q(A)=2 N \\
& A I(A B)\rangle=0
\end{aligned}
$$

where $\mathrm{N}$ is the number of occupied MO's. Not only are these definitions straightforward and precise, but it may even be that the concepts they represent cannot be established with total precision unless overlap is neglected. 
Both theories are fast and manageable for polyatomic calculations involving first row atoms; as manifest by their wide application to topics such as eiectric moments $(20,66-68)$, rotation barriers $(18,19,29,60,69,70)$, equilibrium geometries $(11,60,71)$, isomerization energies (72), bond energies $(29,73)$, hydrogen bonding. $(74,75)$, spectra' ${ }^{\prime}(76)$ and spin densities $(11,12)$. Except for the last two, ? where INDO does better, (12), they generally give similar results in good agreement with experiment. However, both give very poor absolute energies (10).

'M. S. Gordon, Fargo, North Dakota. Configuration interaction using INDO. Private communication. 1972. 


\section{GEOMETRY AND HYBRIDIZATION}

\section{Introduction}

Directed valence theory $(77,78)$ is used to take advantage of the intimate connection between geometry and hybridization in organic chemistry (79). Thus, there are tetrahedral (alkyl), trigonal (vinyl) and linear (ethynyl) carbons, the nomenclature specifying both the geometry and partially - hybridization. The latter is only partially specified for several reasons:

1. The relative status of the sigma and pi orbitals is not fixed.

2. The s-character $(80)$ of the linear hybrids is arbitrary, i.e. it corresponds to a free parameter.

3. The absolute orientation of the triple bond is not defined, i.e. they may be rotated freely about the bond axis. A dividend resulting from the present analysis is that these hybridization ambiguities can be settled by reference to the localized orbitals that will be presented later. In case 1 , the LMO's mix the sigma and pi, producing bent 'banana' bonds (6, 7), so when there is unsaturation banana hybrids are used. In case 2, best fit with localized orbitals obtains if the s-character $(80)$ is assigned the value .4779 (it is .5 for pure sp hybrids). In case 3 , the absolute orientation in non-symmetric situations is defined by maximizing overlaps between hybrids and localized MO's.

Geometries and Numberings of Atoms and Hybrid Basis Functions

Figure 1 depects for each molecule the geometrical arrangement of the atoms, carbon hybrid $\mathrm{AO}^{\prime} \mathrm{s}$ and hydrogen $\mathrm{AO}$ 's. The atoms are labeled 
Figure. 1. Geometries and numberings of atoms and hybrid basis functions 


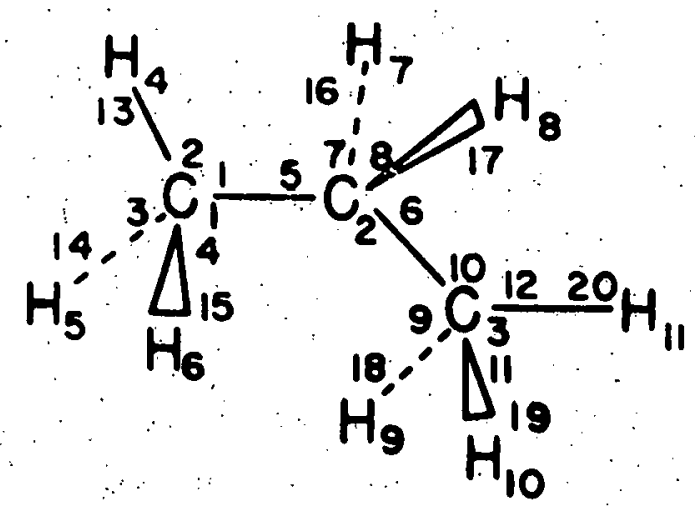

\section{PROPANE}
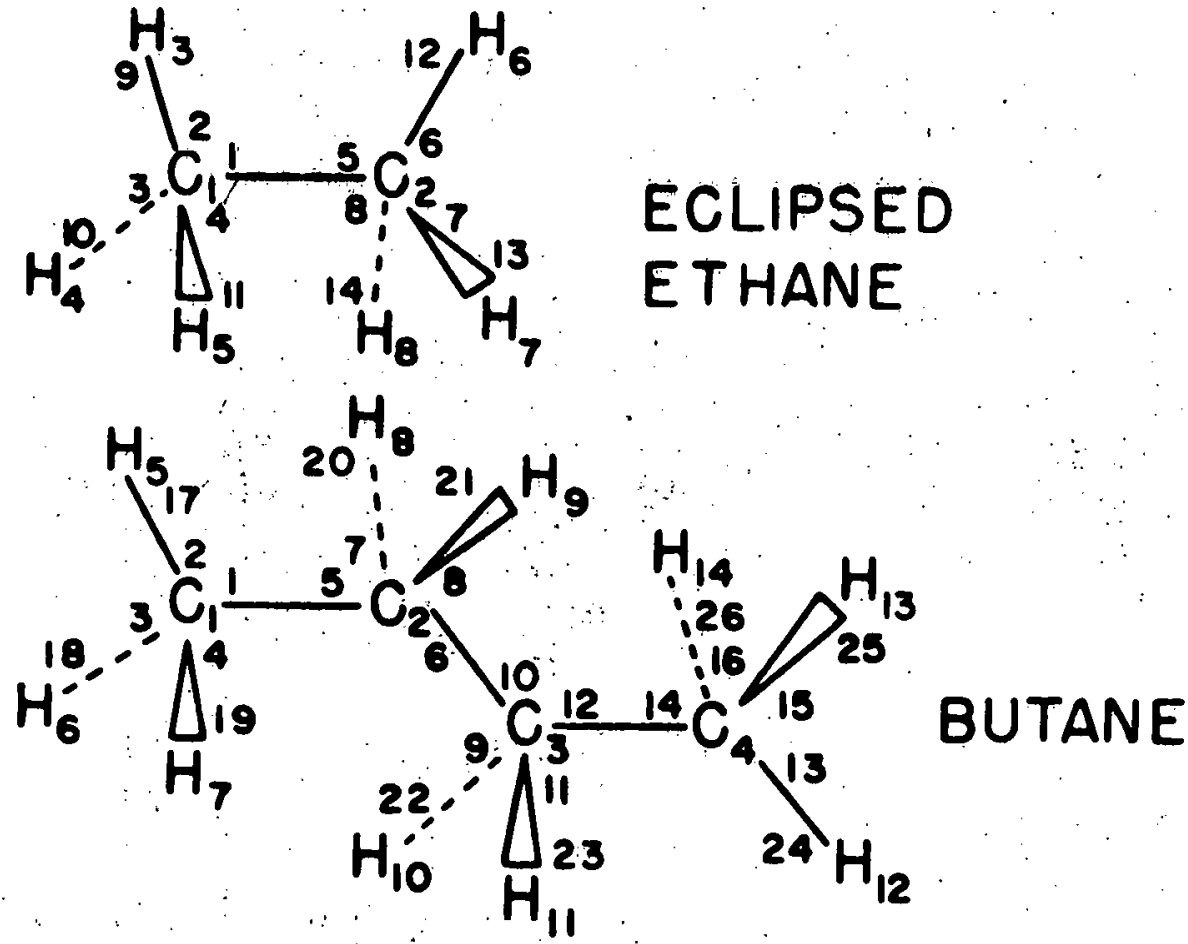


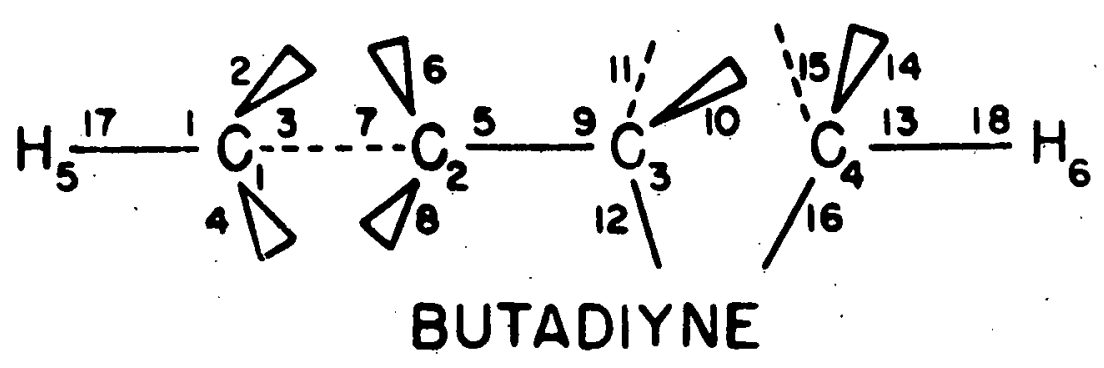

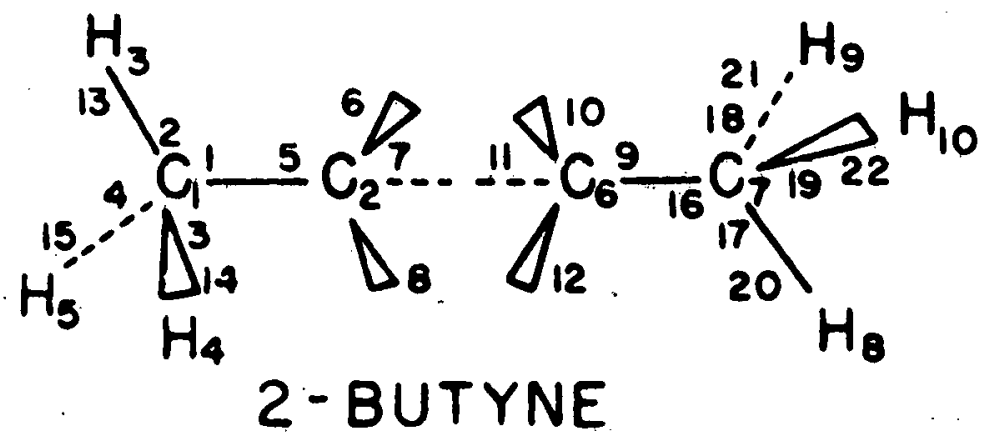

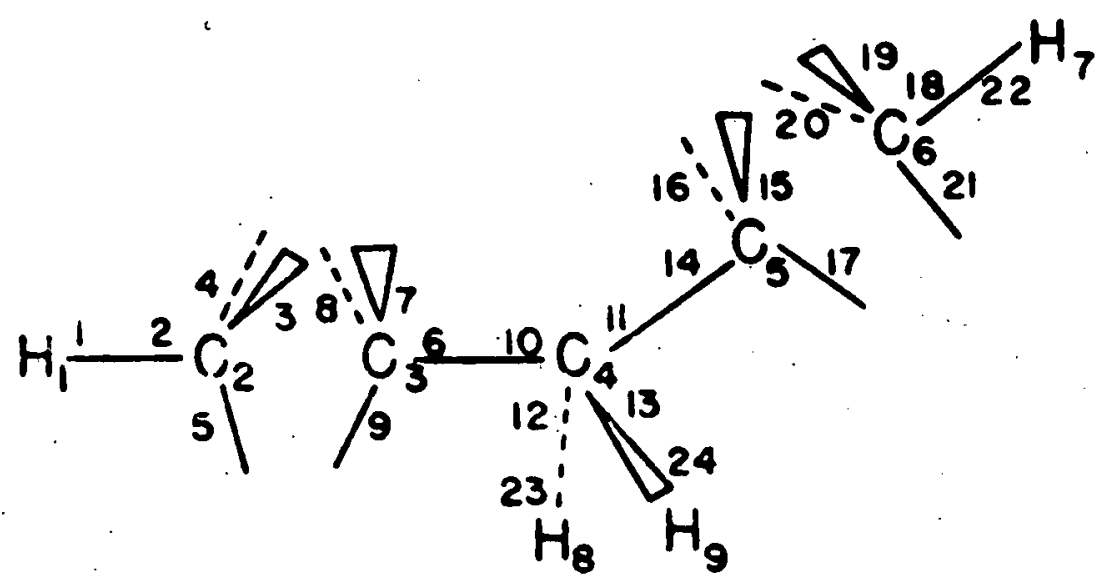

1,4-PENTADIYNE

Figure I (continued) 


$$
\begin{aligned}
& { }_{4}^{7-{ }^{3}-C_{1}} \int_{H_{5}}^{H_{3}} H_{2} \text { METHANE } \\
& \mathrm{H}_{3} \quad \mathrm{C}_{3}^{2 /} \mathrm{C}_{4}^{6} \mathrm{C}_{2}^{5} \mathrm{~b}^{2} \mathrm{H}_{4} \\
& \text { ACETYLENE }
\end{aligned}
$$

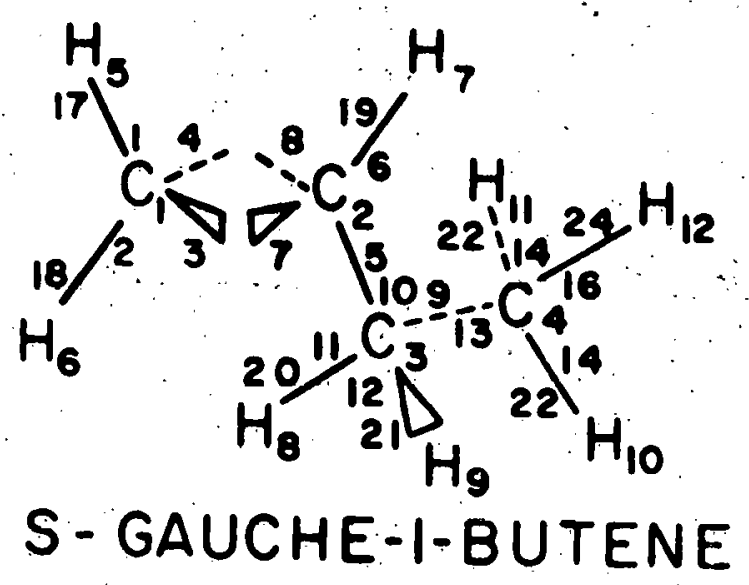

Figure I (Continued) 

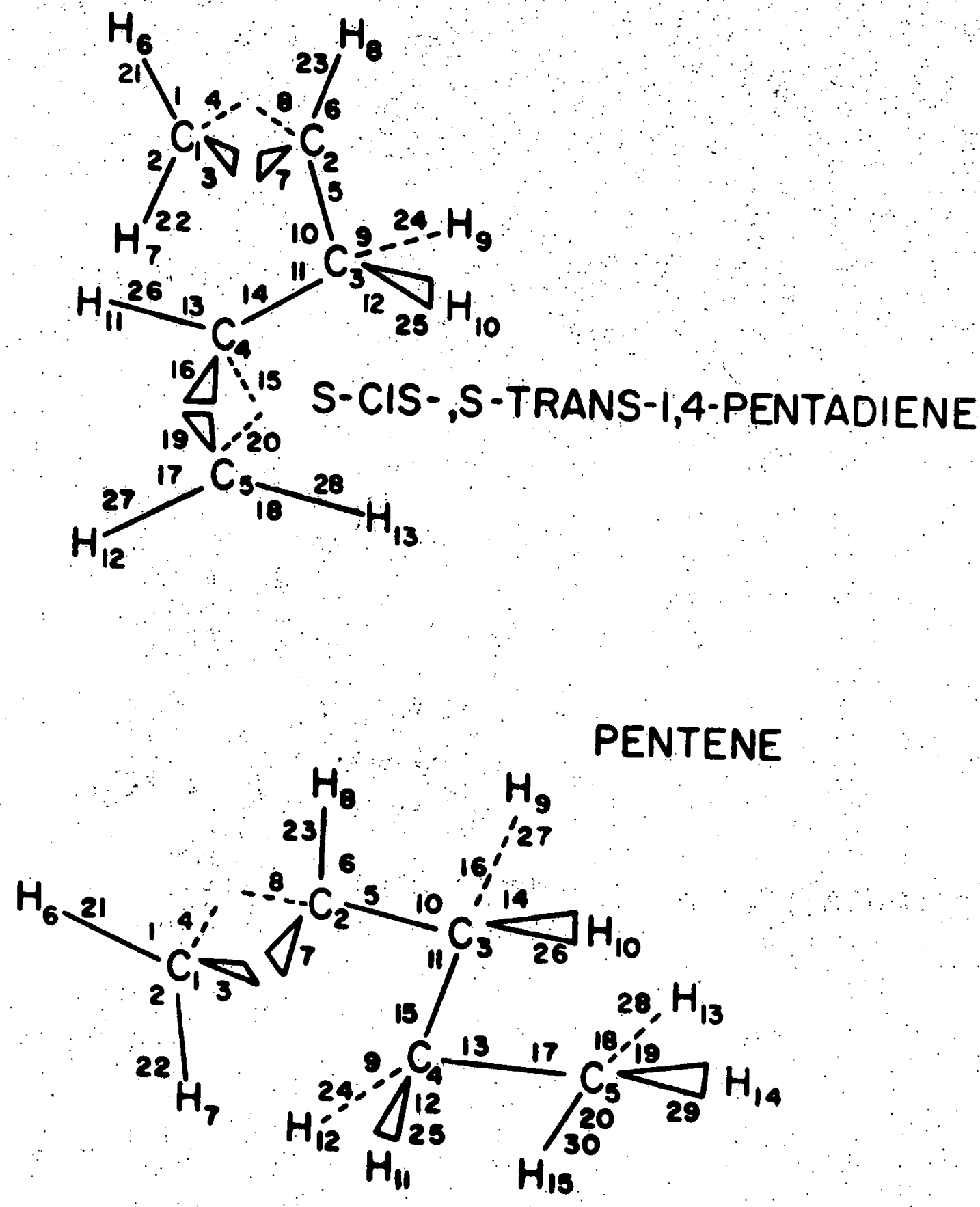

Figure I (continued) 


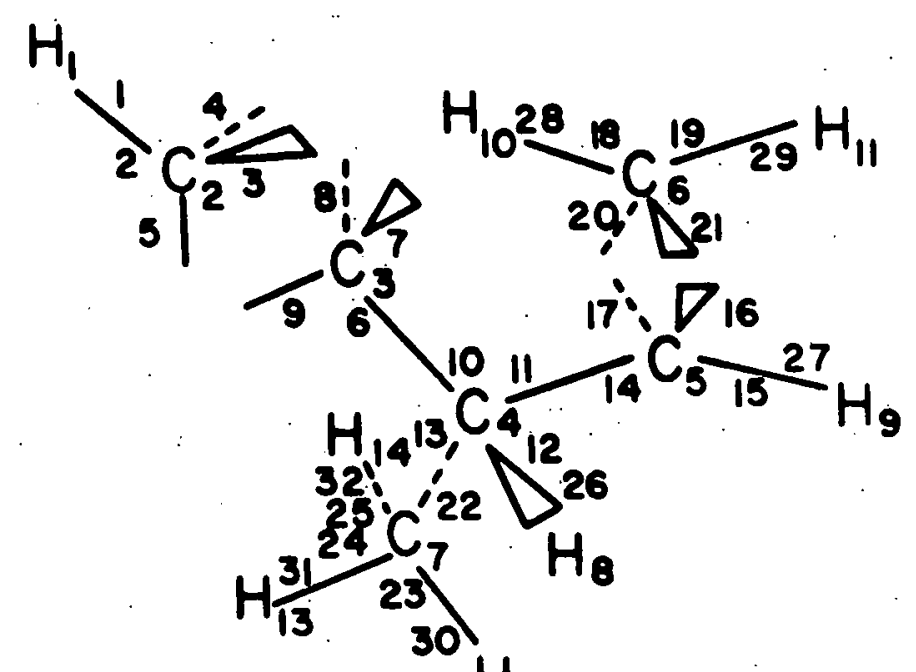

VINYL ETHYNYL ETHANE

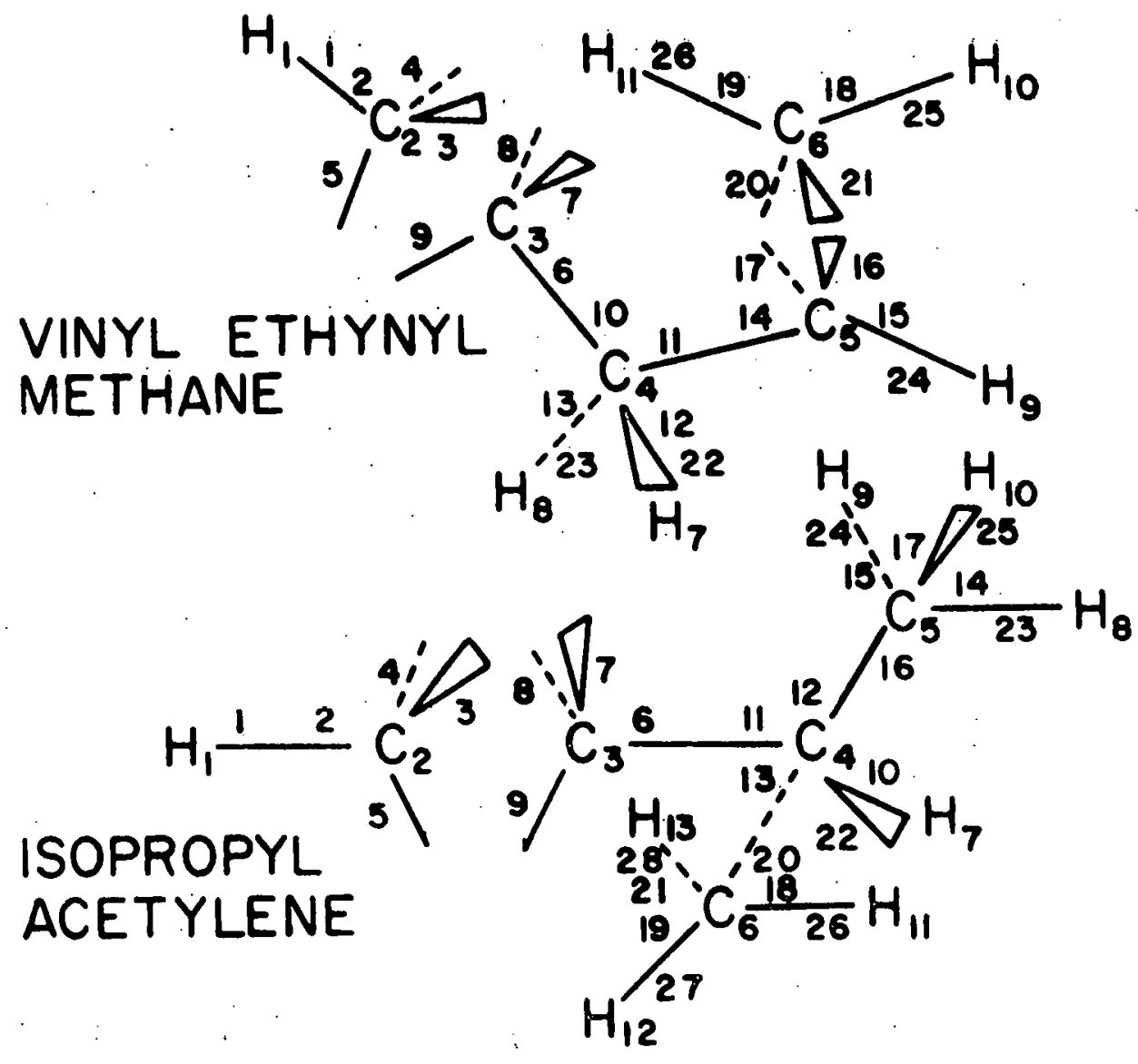

Figure 1 (Continued) 


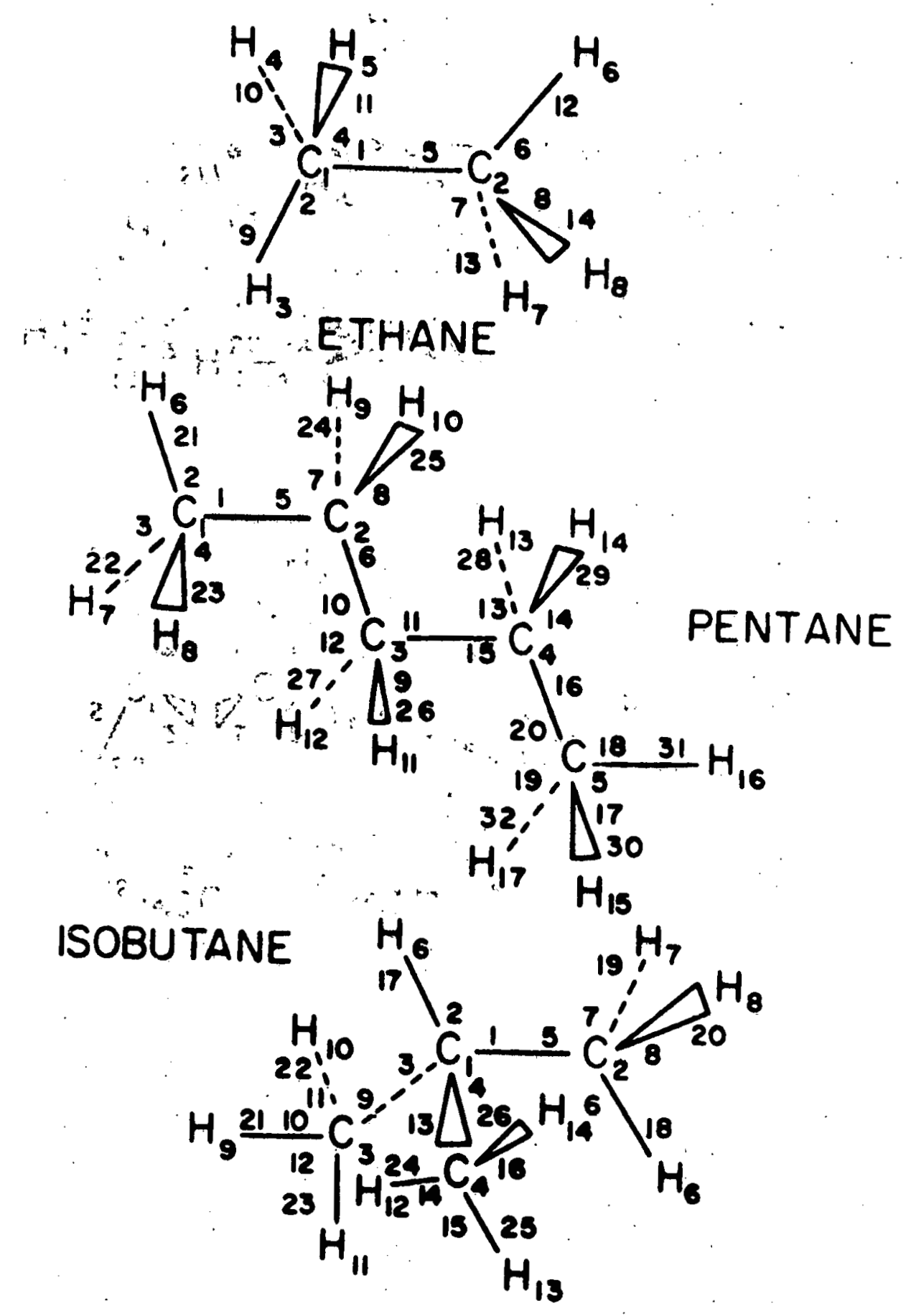

Figure I (Continued) 


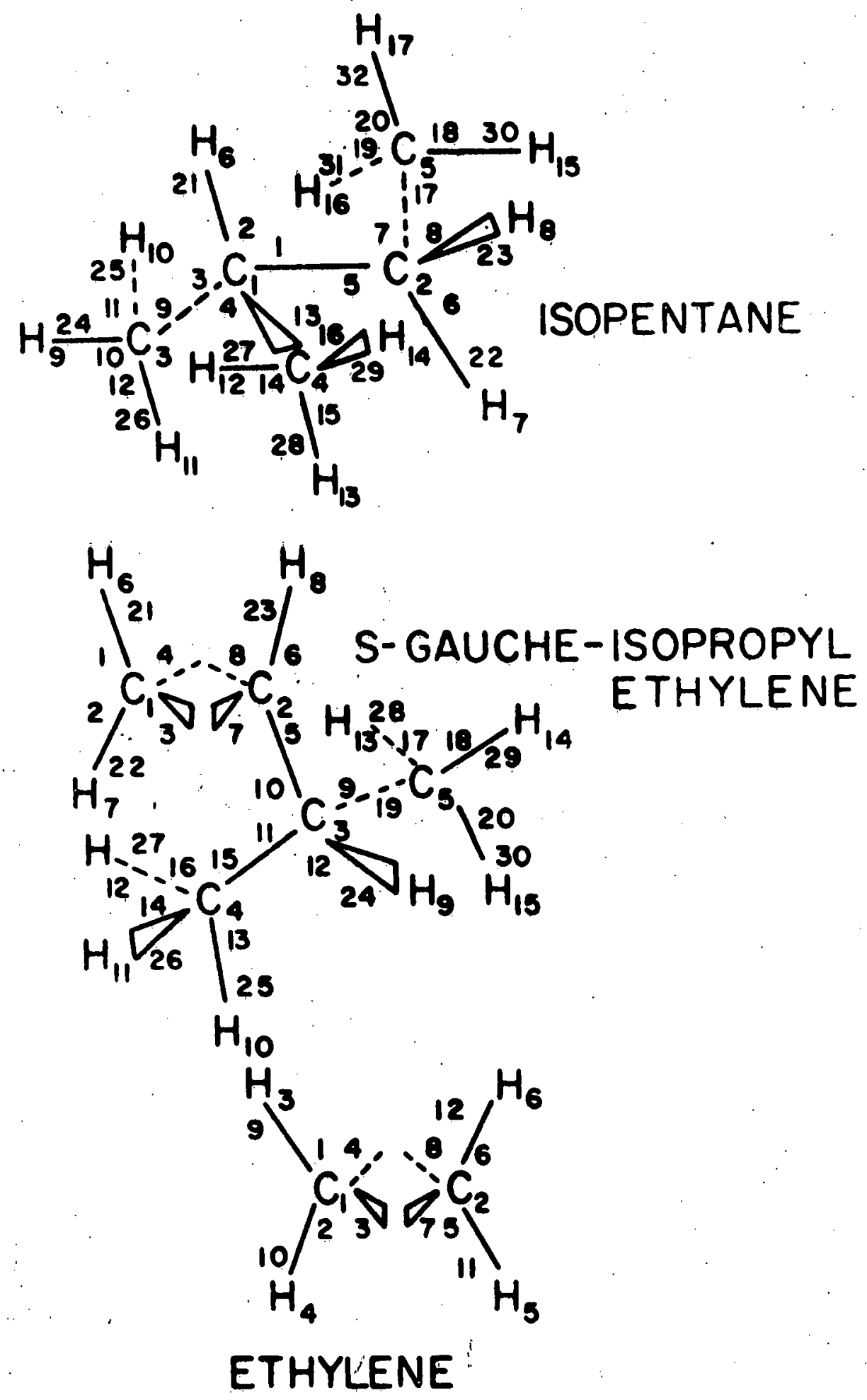

Figure 1 (Continued) 

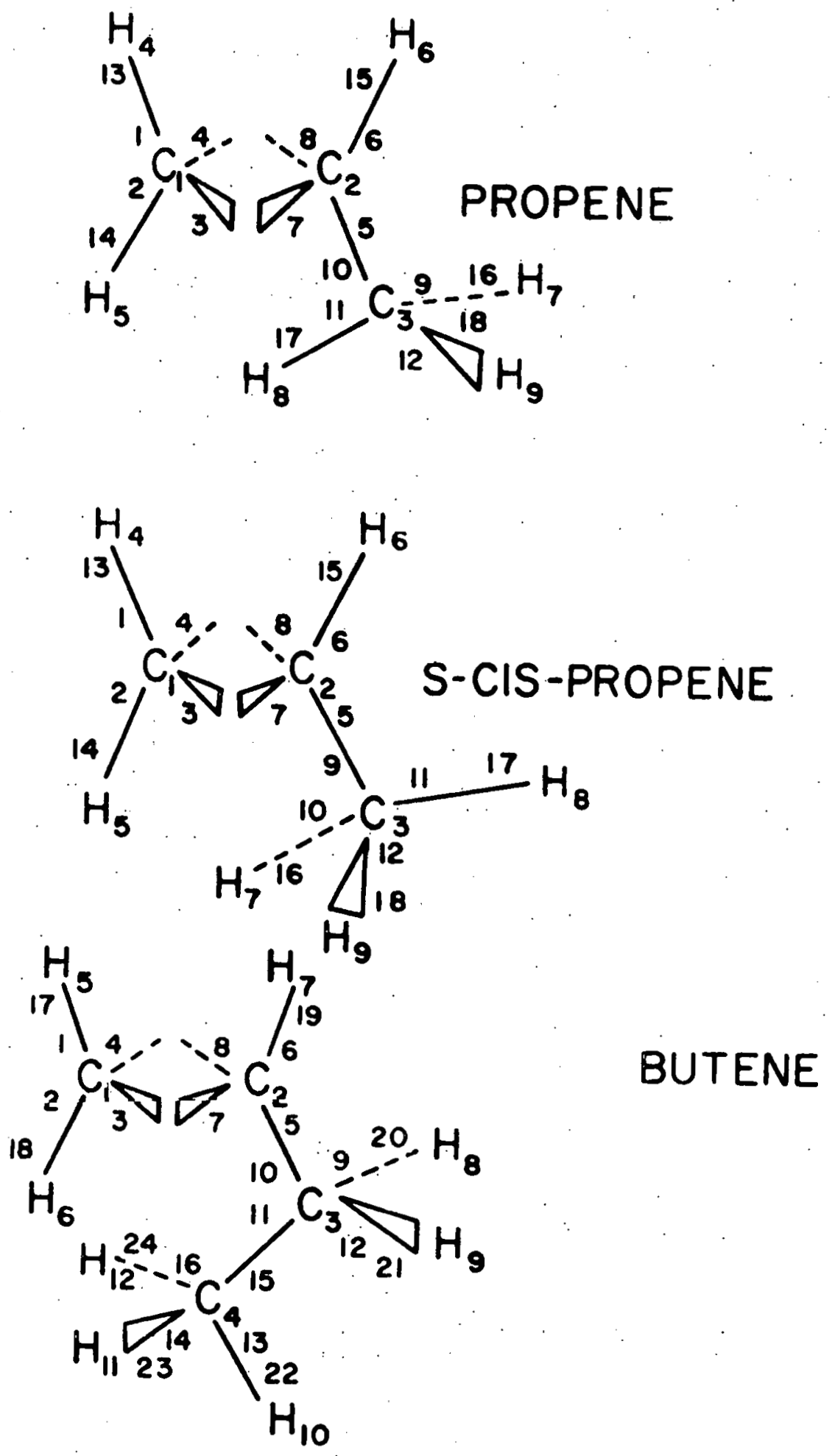

Figure i (Continued) 


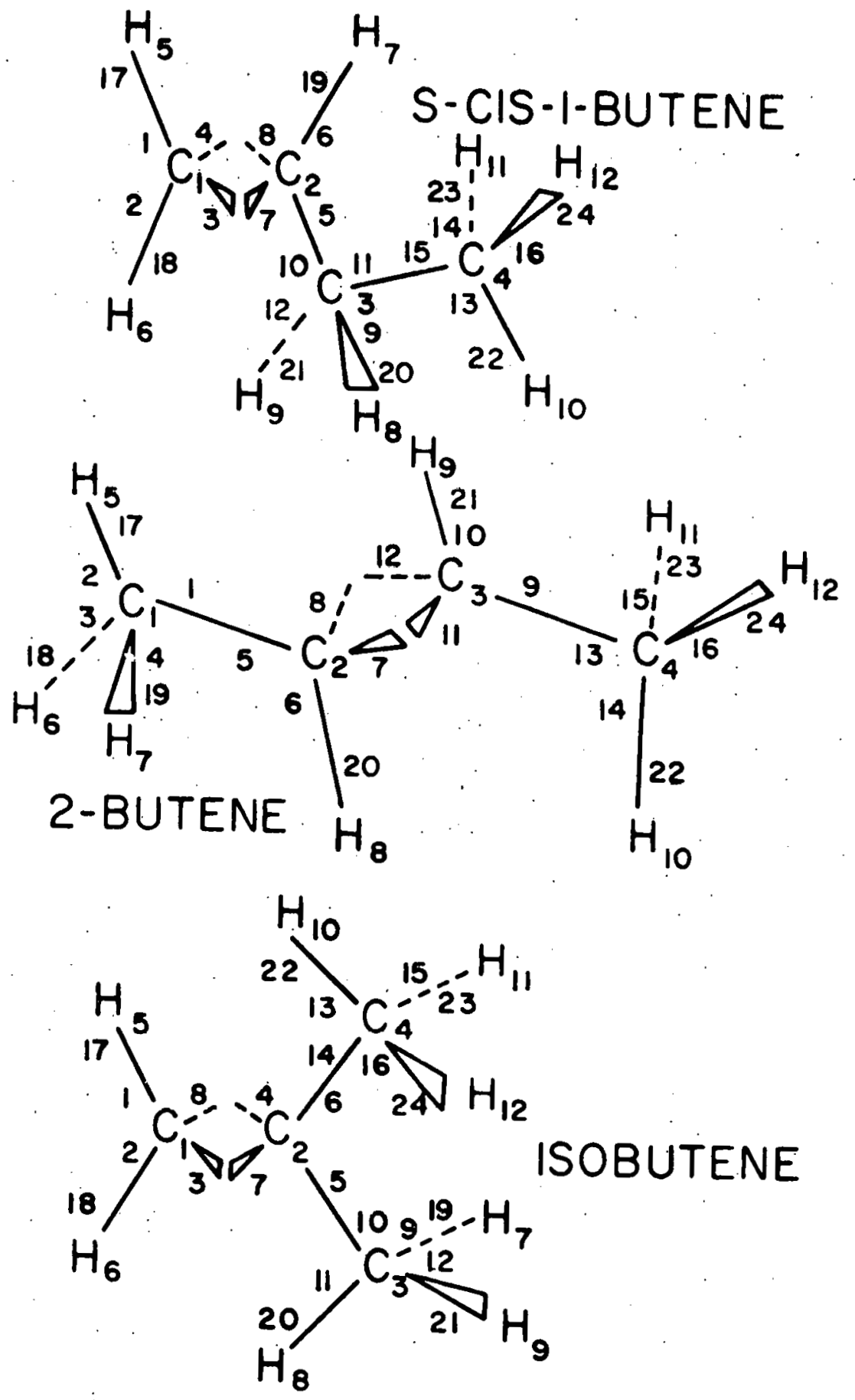

Figure $I$ (Continued) 

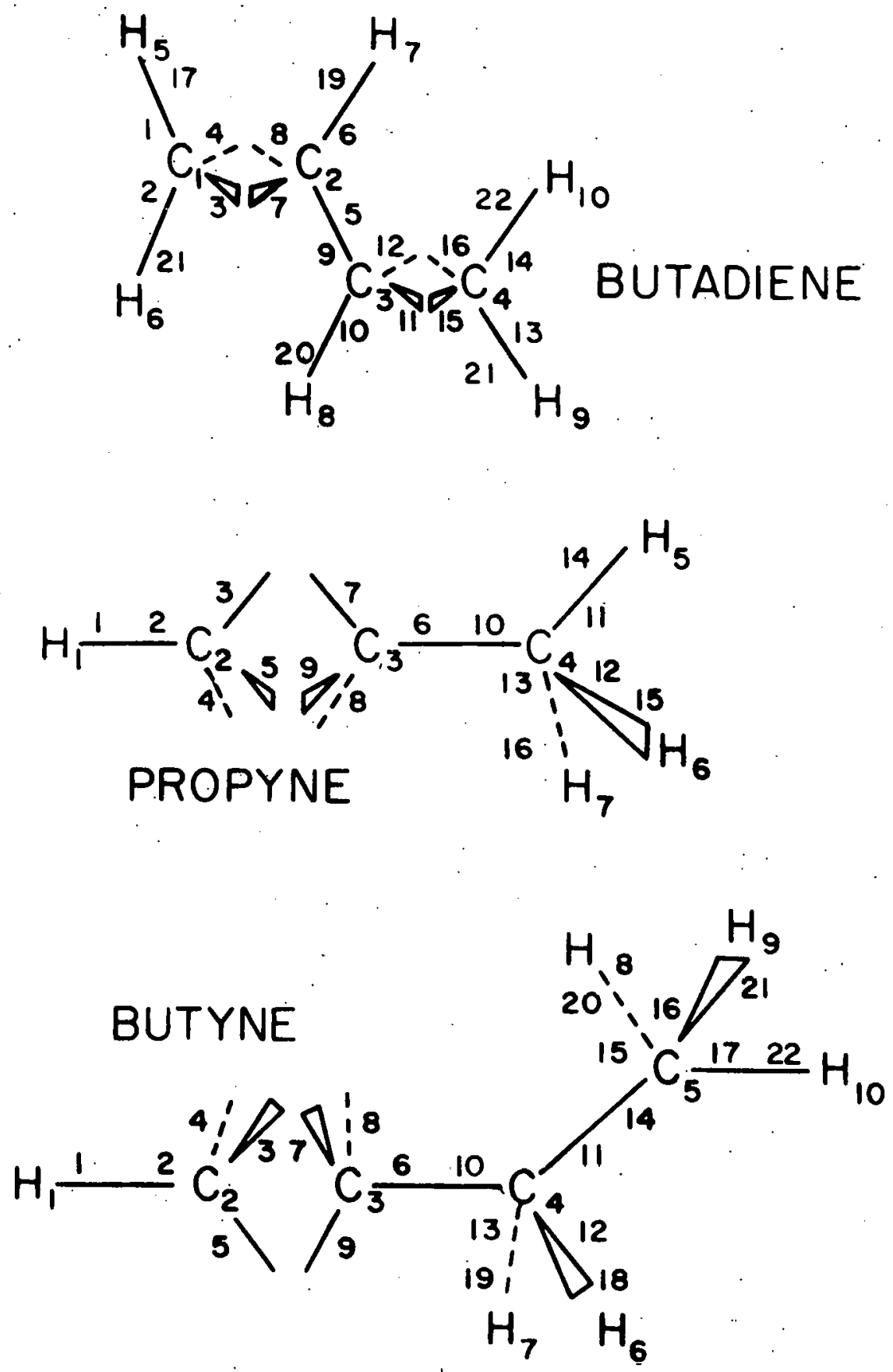

Figure I (Cont inued) 

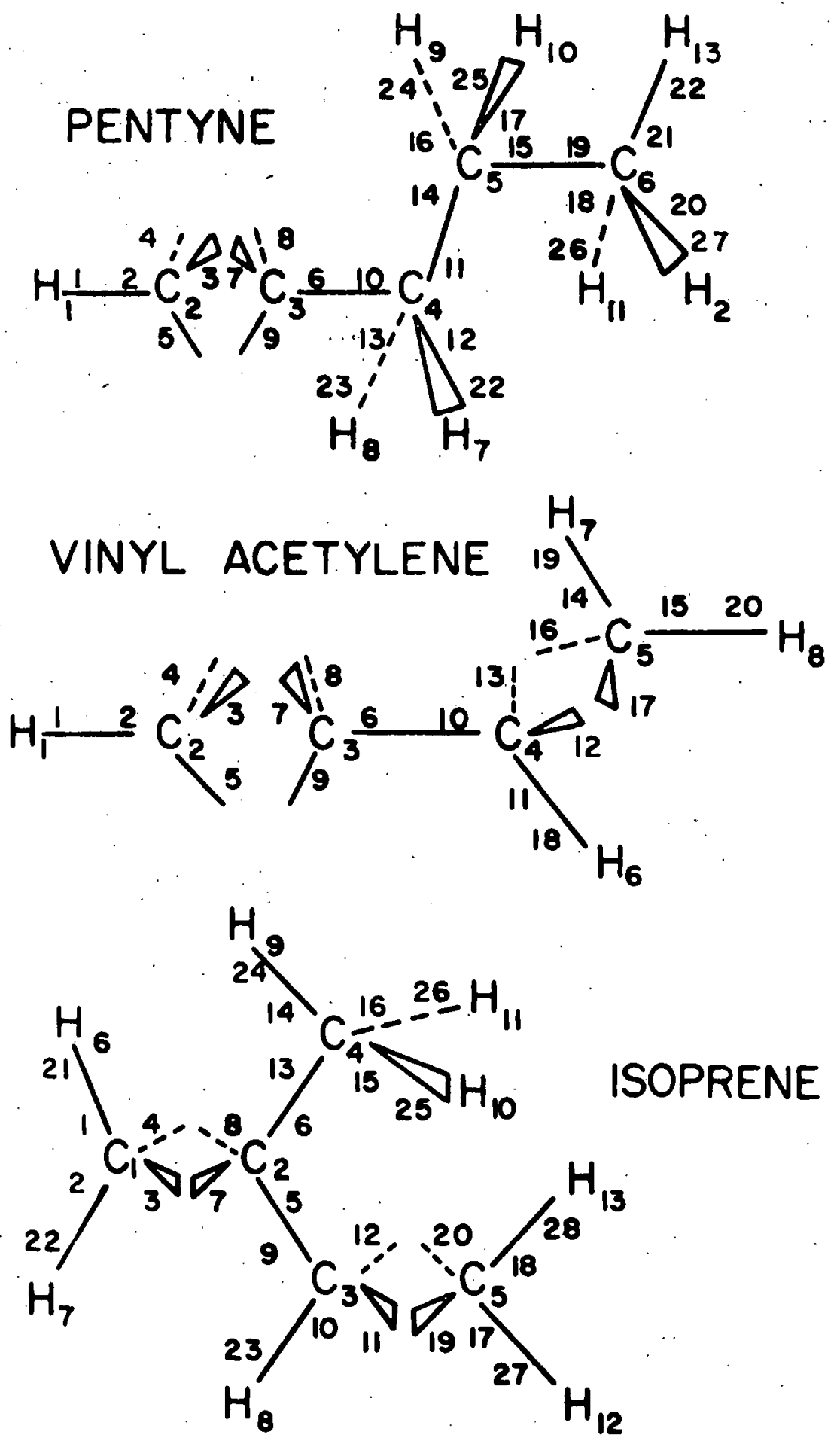

Figure I (Cont inued) 


\section{S-TRANS-ISOPROPYL ETHYLENE}

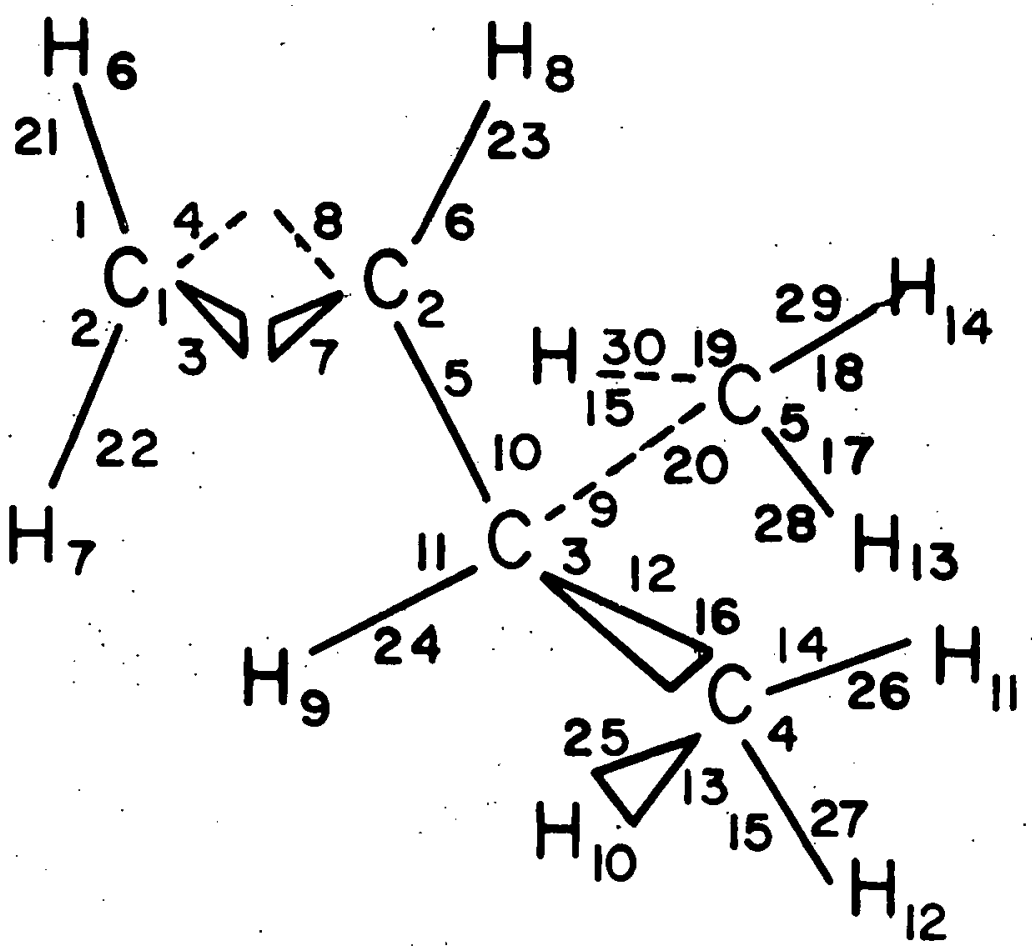

Figure I (Continued) 
$C_{i}, C_{j} \ldots H_{k}, H_{1} \ldots$. The numbers next to each atom label the (hybrid) AO's contributed by that atom. E.g. in propane, the tetrahedral hybrids on the carbon atom $C_{2}$ carry the identification numbers $5,6,7,8$ and the hydrogen is orbital on $\mathrm{H}_{8}$ is labeled number 17. Thus each single bond exhibits at its two ends the labels of its two constituent (hybrid) AO's. Double and triple bonds are depicted by indicating the four or six hybrids that are used to form the two or three banana bonds. Thus the hybrids 3,4 on $C_{1}$ and 7,8 on $C_{2}$ form a double bond in ethylene and the hybrids $2,3,4$ on $C_{1}$ and $6,7,8$ on $c_{2}$ form a triple bond in acetylene.

The cartesian coordinates for these molecules were calculated with the 'Model-Builder' program (47), and appear in Tables 1-33 in Angstrom units. Bondlengths (Table 34) representing averaged experimental values and tetrahedral $\left(109.5^{\circ}\right)$, trigonal $\left(120^{\circ}\right)$ or iinear $\left(180^{\circ}\right)$ angles are assigned automatically by the program, making it especially convenient. These values are basically those adopted in organic chemistry models (79).

Pair Equivalent Hybrids in Muțally Perpendicular Planes

These are two pairs of equivalent hybrids whose centroids lie in mutually perpendicular planes. They will be designated $g(+), g(-)$ and $h(+), h(-)$, with the former lying in the $x z$ plane and the latter in the $y z$ plane. If $A$ and $B$ are the angles measured from the positive and negative z-axes, respectively, toward the positive $x$ - and $y$-axes, respectively, in a right-handed system, then

$$
\begin{aligned}
& g(t)=(s) \cos Q+\sin Q[(z) \cos A \pm(x) \sin A] \\
& h(t)=(s) \cos R+\sin R[(z) \cos B \pm(y) \sin B]
\end{aligned}
$$


TABLE 1. GECMETRY ANC LCAO-MO'S FOR PRCPANE

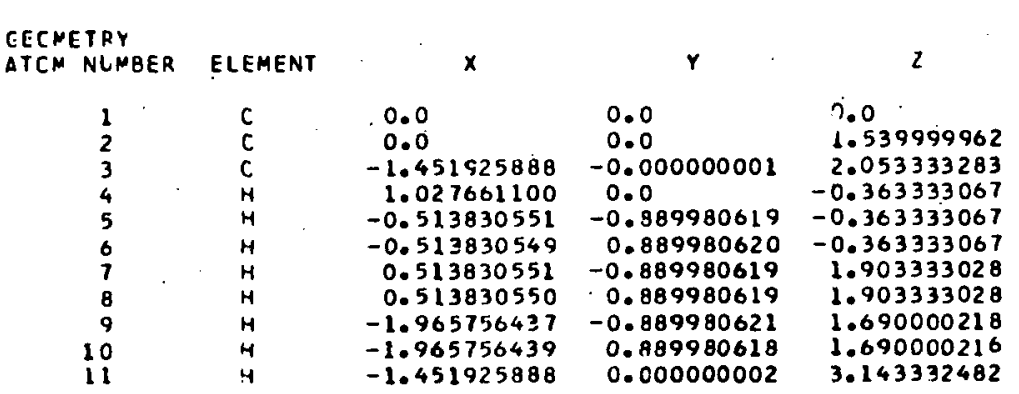

EXPANSIONS IN SLATER BASIS

$\begin{array}{rrrrrrrrrrrr} & & 1 & 2 & 3 & 4 & 5 & 6 & 7 & 8 & 9 & 10 \\ 1 & 2 S & 57 & 226 & -2696 & -3560 & -94 & 3592 & -94 & 3592 & -45 & -57 \\ 1 & P X & -270 & 128 & -55 & -5581 & -47 & -2785 & -47 & -2784 & -540 & 270 \\ 1 & P Y & 443 & 0 & 0 & 0 & -27 & -4842 & 27 & 4843 & 0 & 443 \\ 1 & P 2 & -35 & 262 & -6013 & 2204 & -65 & -2179 & -65 & -2179 & 103 & 35 \\ 2 & 2 S & -3470 & -17 & -2630 & 17 & -74 & -74 & -74 & -74 & 3630 & 3470 \\ 2 & P X & -2975 & 121 & -165 & -380 & -112 & -192 & -112 & -192 & -5634 & 2975 \\ 2 & P Y & 4785 & 0 & 0 & 0 & 437 & -437 & -437 & 437 & 0 & 4785 \\ 2 & P Z & -2103 & 360 & 6035 & 5 & -164 & -51 & -164 & -51 & 2167 & 2103 \\ 3 & 2 S & 57 & 3560 & 45 & -226 & 3592 & -94 & 3592 & -94 & 3696 & -57 \\ 3 & P X & -123 & -218 & 82 & -290 & -2983 & -77 & -2983 & -77 & 5687 & 123 \\ 3 & P Y & 443 & 0 & 0 & 0 & 4842 & 27 & -4842 & -28 & 0 & 443 \\ 2 & P Z & -243 & 5996 & 544 & -33 & -1899 & -23 & -1899 & -23 & -1552 & 243 \\ 4 & 15 & 167 & -138 & 18 & -7136 & 68 & -134 & 68 & -134 & 442 & -167 \\ 5 & \text { is } & 150 & 21 & 10 & 132 & 11 & 7116 & 0 & -120 & -144 & 440 \\ 6 & 15 & -440 & 21 & 10 & 132 & 0 & -120 & 11 & 7116 & -144 & -150 \\ 7 & 15 & -7121 & -158 & 21 & 158 & 425 & -150 & -150 & 425 & -21 & -138 \\ 8 & \text { is } & 138 & -158 & 21 & 158 & -150 & 425 & 425 & -150 & -21 & 7121 \\ 9 & 15 & 150 & -132 & 144 & -21 & -120 & 0 & 7116 & 11 & -10 & 440 \\ 10 & \text { is } & -440 & -132 & 144 & -21 & 1116 & 11 & -120 & 0 & -10 & -150 \\ 11 & 15 & 167 & 7136 & -442 & 138 & -134 & 68 & -134 & 08 & -18 & -167\end{array}$

EXPANSIONS IN HYBRIO BASIS

$\begin{array}{rrrrrrrrrrr} & 1 & 2 & 3 & 4 & 5 & 6 & 7 & 8 & 9 & 10 \\ 1 & -1 & 340 & -7055 & 129 & -103 & -91 & -103 & -91 & 66 & 1 \\ 2 & -181 & 142 & -157 & -6973 & -67 & 151 & -67 & 151 & -494 & 181 \\ 3 & -164 & -14 & -89 & -138 & 10 & 6986 & -28 & 137 & 168 & -462 \\ 4 & 462 & -14 & -89 & -138 & -28 & 137 & 10 & 6986 & 168 & 164 \\ 5 & 86 & -321 & -7041 & 4 & 104 & 7 & 104 & 7 & -62 & -86 \\ 6 & 86 & -4 & 62 & 321 & 1 & 104 & 7 & 104 & 1041 & -86 \\ 7 & -6941 & 144 & -140 & -144 & -440 & 178 & 178 & -440 & 140 & 173 \\ 8 & -173 & 144 & -140 & -144 & 178 & -440 & -440 & 178 & 140 & 6941 \\ 9 & -164 & 138 & -168 & 14 & 137 & -28 & 6986 & 10 & 89 & -462 \\ 10 & -1 & -128 & -66 & -340 & -91 & -103 & -91 & -103 & 1055 & 1 \\ 11 & 442 & 138 & -168 & 14 & 6986 & 10 & 137 & -28 & 90 & 164 \\ 12 & -181 & 6973 & 494 & -142 & 151 & -67 & 151 & -67 & 157 & 181 \\ 12 & 167 & -138 & 188 & -7136 & 68 & -134 & 68 & -134 & 442 & -167 \\ 14 & 150 & 21 & 10 & 132 & 11 & 7116 & 0 & -120 & -144 & 440 \\ 15 & -440 & 21 & 10 & 132 & 0 & -120 & 11 & 7116 & -144 & -150 \\ 16 & -7121 & -158 & 21 & 158 & 425 & -150 & -150 & 425 & -21 & -138 \\ 17 & 138 & -158 & 21 & 158 & -150 & 425 & 425 & -150 & -21 & 7121 \\ 18 & 150 & -132 & 144 & -21 & -120 & 0 & 7116 & 11 & -10 & 440 \\ 19 & -440 & -132 & 144 & -21 & 7116 & 11 & -120 & 0 & -10 & -150 \\ 20 & 167 & 7136 & -442 & 138 & -134 & 68 & -134 & 68 & -18 & -167 \\ -130 & -136 & -130\end{array}$


tABLE 2. GECMETRY ANC LCAO-MO'S FCR ECLIPSEO ETHANE

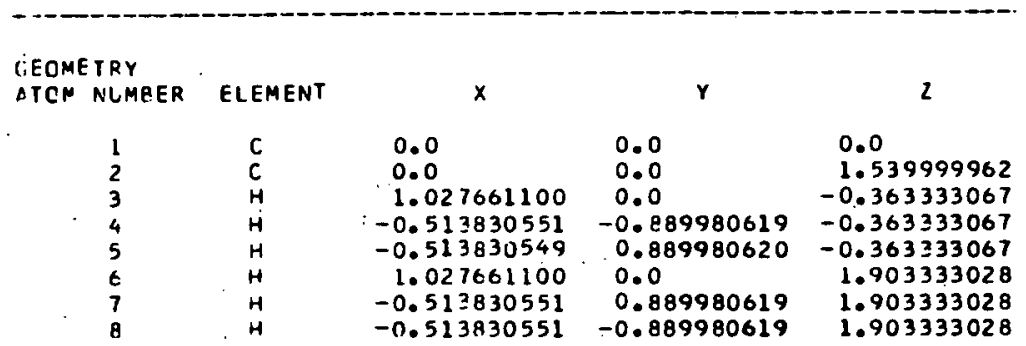

EXPANSIONS IN SLATER BASIS

$\begin{array}{rrrrrrrrr} & & 1 & 2 & 3 & 4 & 5 & 6 & 7 \\ 1 & 2 S & 56 & -56 & 3692 & -3577 & 56 & 3577 & 3577 \\ 1 & P X & 252 & -252 & 0 & 2795 & -504 & 5590 & -2795 \\ 1 & P Y & -437 & -437 & 0 & -4841 & 0 & 0 & -4841 \\ 1 & P 2 & -41 & .41 & 6030 & 2189 & -41 & -2189 & -2191 \\ 2 & 2 S & -3577 & 3577 & 3692 & 57 & -3577 & -56 & -55 \\ 2 & P X & 2795 & -2795 & 0 & 252 & -5590 & 504 & -252 \\ 2 & P Y & -4841 & -4841 & 0 & -437 & 0 & 0 & -437 \\ 2 & P 2 & -2190 & 2189 & -6030 & 41 & -2189 & -41 & -41 \\ 3 & 1 S & -237 & 237 & -6 & 122 & 346 & 7115 & -123 \\ 4 & 15 & -237 & -347 & -5 & 123 & -237 & -123 & 7115 \\ 5 & 15 & 346 & 237 & -6 & -7115 & -237 & -122 & -123 \\ 6 & 1 S & 123 & -122 & -6 & -238 & -7115 & -346 & 237 \\ 7 & 15 & -7115 & -122 & -6 & 346 & 123 & 237 & 237 \\ 8 & 1 S & 123 & 7115 & -6 & -238 & 122 & 237 & -347\end{array}$

EXPANSICNS IN HYBRID BASIS

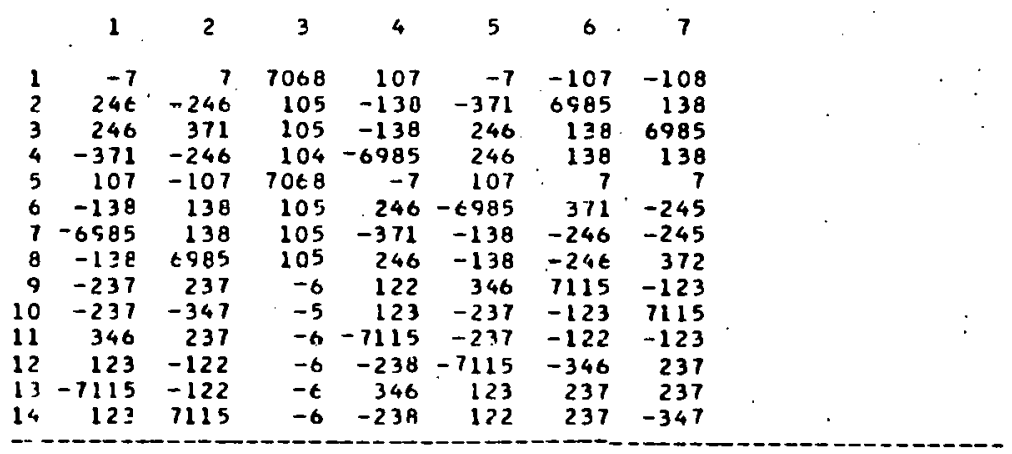


TAMLE 3. GECMETRY ANC LCAO-MO'S FOR BUTANE

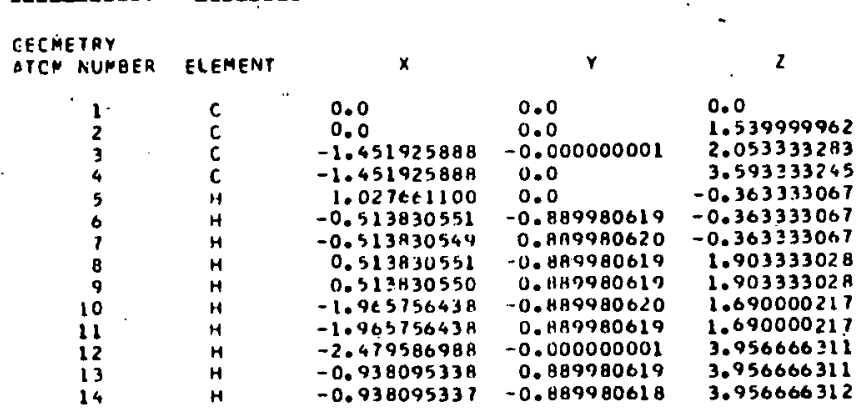

EXPANSIONS IN SLATER BASIS

\begin{tabular}{|c|c|c|c|c|c|c|c|c|c|c|c|c|c|c|}
\hline & & 1 & 2 & 3 & 4 & 5 & $t$ & 7 & B & 9 & 10 & 11 & 12 & 13 \\
\hline & 25 & & $\begin{array}{l}-51 \\
548\end{array}$ & $\begin{array}{l}-68 \\
-47\end{array}$ & $\begin{array}{l}-35 \\
-55\end{array}$ & $\begin{array}{l}-98 \\
-50\end{array}$ & $\begin{array}{r}3594 \\
-2705\end{array}$ & $\begin{array}{r}35 \\
-278\end{array}$ & $\begin{array}{r}34 \\
4\end{array}$ & $\begin{array}{l}-98 \\
-50\end{array}$ & $\begin{array}{l}223 \\
136\end{array}$ & $\begin{array}{r}34 \\
4\end{array}$ & $\begin{array}{r}-37 c 8 \\
-70\end{array}$ & $\begin{array}{l}-64 \\
265\end{array}$ \\
\hline 1 & $\begin{array}{l}P X \\
P Y\end{array}$ & $\begin{array}{r}10.3 \\
442\end{array}$ & & 0 & & $\begin{array}{l}50 \\
25\end{array}$ & 4841 & -4841 & -14 & -25 & 0 & 14 & 0 & $\begin{array}{l}203 \\
442\end{array}$ \\
\hline$i$ & PZ & -26 & 100 & -89 & 2217 & -70 & -2174 & -217 & & -70 & 257 & 55 & -6022 & $2 t$ \\
\hline 2 . & 25 & 3484 & 363 & 211 & 20 & -79 & -75 & -7 & & -7 & -5 & -94 & & 3484 \\
\hline 2 & $p x$ & 2961 & & & -38 & -212 & -293 & -19 & 77 & -121 & 71 & 77 & -182 & 2961 \\
\hline 2 & or & 4789 & & 0 & 0 & -432 & 438 & -43 & -25 & 4 & 0 & 25 & & 4789 \\
\hline 2 & $P Z$ & -2105 & 2117 & 4 & 1 & -17 & -52 & -5 & 3 & -17 & 396 & 13 & 6035 & 2105 \\
\hline 3 & 25 & & 363 & -20 & -217 & 3484 & -94 & -9 & -75 & 3484 & 36 & -75 & 5 & -79 \\
\hline 3 & $P x$ & -111 & 5636 & $3 \mathrm{B2}$ & -286 & -290 & -7 & -7 & 193 & -2561 & 18 & 193 & 71 & 111 \\
\hline 3 & $\hat{p Y}$ & 432 & & 0 & 0 & -4789 & -25 & 25 & 438 & 4789 & 0 & -438 & 0 & 432 \\
\hline 3 & P2 & -172 & -2117 & 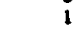 & 4 & -2105 & -13 & -13 & 52 & -2105 & 9 & 52 & 396 & 172 \\
\hline 4 & 25 & & -51 & 3552 & 68 & -64 & 34 & 34 & 594 & -64 & 3708 & 594 & & -98 \\
\hline & $p x$ & -50 & 548 & -557 & -47 & -26 & -4 & -4 & 35 & -265 & -70 & 278 & $13 t$ & 50 \\
\hline & PY & -2.5 & & & 0 & -442 & -14 & 14 & 8 & 442 & 0 & -484 & 0 & -25 \\
\hline 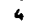 & $P Z$ & -70 & -100 & 2217 & -89 & -26 & -55 & -55 & 2174 & -20 & -6022 & 217 & 257 & 70 \\
\hline & is & 162 & 446 & 59 & -7138 & 33 & -135 & -135 & -1 & 72 & -1 & -1 & 20 & -162 \\
\hline 6 & 15 & 151 & -144 & -14 & 132 & -2 & $-1 \geqslant 1$ & 7117 & -8 & 11 & 2 & & i1 & 439 \\
\hline 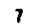 & 15 & -439 & -14 & -14 & 13 & 11 & 7117 & -12 & 12 & -2 & 20 & -8 & ii & -151 \\
\hline & 15 & -7121 & -2 & 2 & is6 & -142 & 426 & $-i 5 i$ & 11 & 425 & -1 & -1 & 29 & -136 \\
\hline 9 & is & 136 &.-27 & 21 & 150 & 425 & -151 & 426 & $-i$ & -142 & -145 & 11 & 29 & 1121 \\
\hline & is & 142 & -27 & -156 & -21 & 112 & ii & -1 & 426 & & $-z$ & -15 & & 425 \\
\hline & is & -425 & $-2 i$ & $-i$ & -21 & -13 & & 11 & -151 & & -29 & 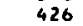 & & -142 \\
\hline & 15 & -72 & 446 & 1138 & -58 & -16 & -1 & -10 & -135 & -1 & -20 & -1 & 134 & 12 \\
\hline & is & -11 & & 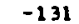 & 1 & & i & -8 & 7117 & -25 & -11 & -12 & - & -2 \\
\hline & is & 2 & 4 & 11 & 14 & -151 & -8 & 12 & -121 & 439 & -11 & 7117 & -20 & 11 \\
\hline
\end{tabular}

EXPANSICNS IN TYBRIO BASIS

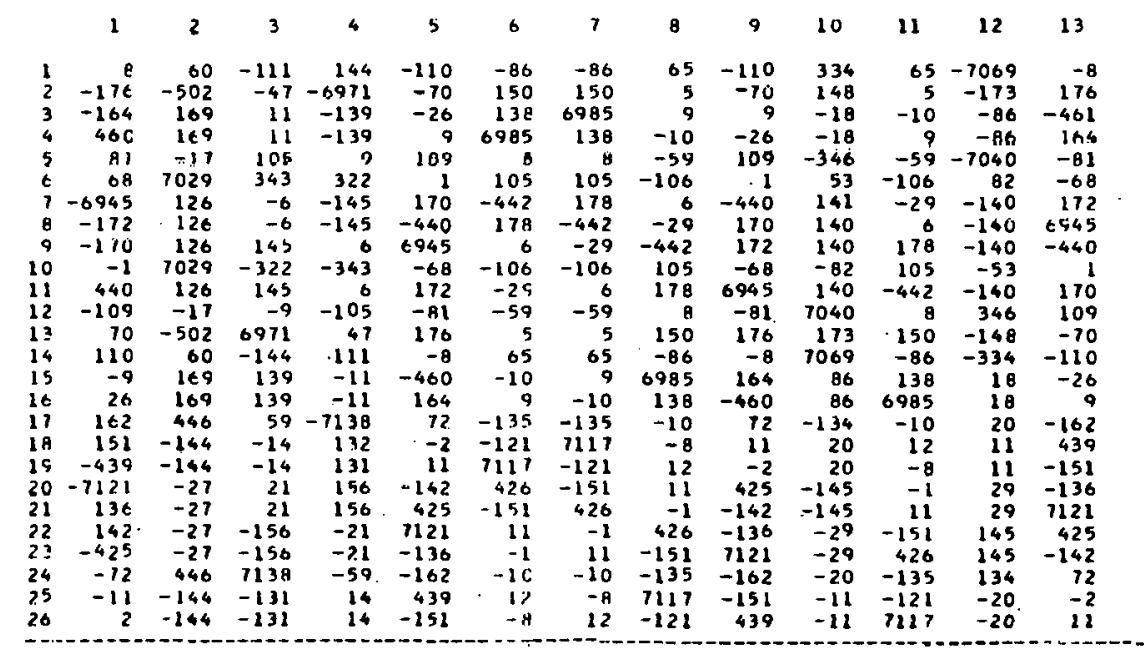


TABLE 40 GECMETRY ANC LCAD-MDIS FCR BUTAOIYNE

GECMETRY

atc nUMAER

ELEMENT

$x$

$r$

z

$\begin{array}{ll}1 & \\ 2 & \\ 3 & \\ 4 & \\ 5 & \\ 6 & \end{array}$
0.0
0.0
-0.000
$-0.000000000$
$-0.000000000$
$-0.000000000$

0.0
0.0
0.0
0.0
0.0
0.0

0.0

1.199599807

2.579998970

3.779958779

$-1.059599466$

4.839998245

EXPANSICNS IN SLATER BASIS

\begin{tabular}{|c|c|c|c|c|c|c|c|c|c|c|}
\hline & & 1 & 2 & 3 & 4 & 5 & 6 & 7 & 8 & 9 \\
\hline & 25 & 2262 & 19 & 540 & 3262 & -79 & 4970 & -3262 & 79 & \\
\hline & & & 434 & & & & 0 & & 82 & \\
\hline & PY & & 690 & 0 & & -1 & 0 & & & \\
\hline & C2 & 2 & & 62 & & -31 & -5361 & -2 & & \\
\hline & $2 S$ & & -1 & -47 & & 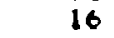 & & & & \\
\hline & $\nabla x$ & -5 & 439 & 0 & $-4 c$ & -384 & 0 & 446 & -82 & \\
\hline & Pr & -2 & 6 & 0 & & 7 & 0 & 56 & & \\
\hline & 02 & 258 & 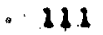 & -5211 & & -11 & $2 \mathrm{C7}$ & 2582 & 1 & 18 \\
\hline & 25 & & & & & -31 & & 16 & & \\
\hline & $P x$ & -74 & 30 & 0 & -679 & -26 & 0 & 64 & .56 & \\
\hline & PY & -35 & 48 & 0 & 467 & & 0 & 822 & 21 & \\
\hline 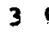 & 02 & & 25 & 5211 & -111 & -25 & -146 & 111 & & \\
\hline & 25 & - & 32 & & & -3 & -13 & -79 & 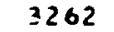 & 87 \\
\hline & $F x$ & 736 & 30 & 0 & 672 & -2 & 0 & -64 & -5 & \\
\hline & Dr & 351 & & 0 & -462 & & 0 & -813 & & \\
\hline & D2 & 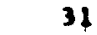 & 24 & , & -31 & 2 & $=$ & 31 & 8 & 36 \\
\hline & 15 & 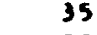 & & & & 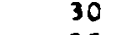 & 6860 & 35 & -30 & \\
\hline & is & 30 & -35 & 337 & -30 & 35 & 42 & 30 & -35 & 26 \\
\hline
\end{tabular}

EXPANSICNS IN HYORIO EASIS

\begin{tabular}{|c|c|c|c|c|c|c|c|c|c|}
\hline & ! & 2 & 3 & 4 & .5 & 6 & 7 & 8 & 9 \\
\hline 1 & -372 & 29 & 333 & 311 & -29 & 7243 & -371 & 29 & -27 \\
\hline 2 & -7044 & -517 & 247 & 7 & -19 & -0 & & 636 & -43 \\
\hline 3 & -7 & t $3 t$ & 247 & 7 & 517 & -6 & -7044 & 19 & -43 \\
\hline 4 & -7 & 19 & 247 & 7044 & -636 & -6 & -1 & -517 & -43 \\
\hline 5 & -205 & 72 & -7041 & 209 & -71 & -245 & -209 & 71 & 250 \\
\hline$\epsilon$ & -6987 & 519 & $\epsilon$ & 25 & 23 & $-3,33$ & -26 & $-64 t$ & 32 \\
\hline 7 & -25 & -646 & 6 & 25 & -518 & -333 & -6987 & -23 & 32 \\
\hline 8 & $-2 t$ & -23 & 6 & 6987 & 646 & -333 & -25 & 519 & 32 \\
\hline 9 & -71 & 209 & -7061 & 71 & -209 & 250 & -71 & 209 & -245 \\
\hline 10 & -519 & 6987 & 6 & -23 & -26 & $3 ?$ & 646 & 25 & -333 \\
\hline 12 & 23 & 25 & 6 & -646 & -6987 & 32 & -519 & 25 & -333 \\
\hline 12 & 646 & 25 & 6 & 519 & -25 & 32 & 23 & 6987 & -333 \\
\hline 13 & -25 & 371 & 333 & 29 & -371 & -27 & -29 & 371 & 7243 \\
\hline 14 & 527 & 1044 & 267 & 19 & -7 & -43 & -636 & 7 & -6 \\
\hline 15 & -15 & 7 & 247 & 636 & -7044 & -43 & 517 & 7 & -6 \\
\hline 16 & $-t 3 t$ & 7 & 267 & -517 & -7 & -43 & -19 & 7044 & -6 \\
\hline 17 & 35 & -30 & $-33 ?$ & -35 & 30 & 0860 & 35 & -30 & 42 \\
\hline 18 & 30 & -35 & -337 & -30 & 35 & 42 & 30 & -35 & 6860 \\
\hline
\end{tabular}


TABLE 5. GEOMETRY AND LCAO-MO'S FCR 2-RUTYNE

CEONETRYY

ATCW NUYBER ELEMENT

1
2
3
4
5
6
7
8
9
10

$C$
$C$
$H$
$H$
$H$
$C$
$C$
$H$
$H$
$H$

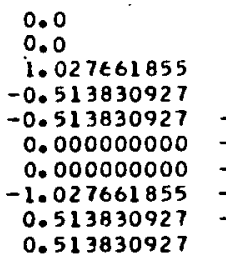

0.0
0.0

0.0

0.889981274

$-0.889981274$

$-0.000000000$

$-0.000000000$

$-0.000000000$

0.889981274

0.0

1.460600000

$-0.363333333$

$-0.363333333$

0.363333333

2.660000000

4.120000000

4.483333333

4.483333333

EXPANSICNS IN SLATER BASIS

$\begin{array}{rrrrrrrrrrrrr} & & 1 & 2 & 3 & 4 & 5 & 6 & 7 & 8 & 5 & 10 & 11 \\ 1 & 2 S & -29 & 17 & -3533 & -3532 & 171 & 29 & -17 & -17 & 29 & -3673 & -3532 \\ 1 & P X & 525 & -83 & 2786 & 2786 & 0 & 61 & -41 & -41 & 463 & 0 & -5573 \\ 1 & P Y & 232 & 0 & 4826 & -4826 & 0 & 570 & -71 & 71 & -338 & 0 & 0 \\ 1 & P Z & 67 & 20 & 2240 & 2240 & 220 & -67 & -20 & -20 & -67 & -5946 & 2240 \\ 2 & 25 & 3192 & -92 & 58 & 58 & -563 & -3192 & 92 & 92 & -3192 & -4784 & 58 \\ 2 & P X & 5228 & -699 & 375 & 375 & 0 & 613 & -349 & -349 & 4615 & 0 & -751 \\ 2 & P Y & 2310 & 0 & 650 & -650 & 0 & 5683 & -605 & 605 & -3373 & 0 & 0 \\ 2 & P Z & 2541 & -48 & 107 & 107 & -143 & -2541 & 48 & 48 & -2541 & 5275 & 107 \\ 3 & 15 & -383 & 133 & 103 & 103 & -22 & -93 & 72 & 72 & -419 & -18 & -7091 \\ 4 & 15 & 93 & -72 & 103 & -7052 & -22 & -419 & 72 & -133 & 383 & -18 & 103 \\ 5 & 15 & 419 & -72 & -7092 & 103 & -22 & 383 & -133 & 72 & -53 & -18 & 103 \\ 6 & 25 & 3192 & 59 & -92 & -92 & 4784 & -3192 & -59 & -59 & -3192 & 563 & -92 \\ 6 & P X & 5228 & 751 & -349 & -349 & 0 & 613 & 375 & 375 & 4615 & 0 & 699 \\ 6 & P Y & 2310 & 0 & -605 & 605 & 0 & 5683 & 650 & -650 & -3373 & 0 & 0 \\ t & P Z & -2541 & -107 & 48 & 48 & 5275 & 2542 & 107 & 107 & 2541 & -143 & 4 A \\ 7 & 25 & -29 & -3533 & 17 & 17 & 3673 & 29 & 3533 & 3533 & 29 & -171 & 17 \\ 7 & P X & 525 & 5573 & -41 & -41 & 0 & 61 & 2786 & 2786 & 463 & 0 & 83 \\ 7 & P Y & 232 & 0 & -71 & 71 & 0 & 570 & 4826 & -4826 & -338 & 0 & 0 \\ 7 & P Z & -67 & -2240 & -20 & -20 & -5946 & 67 & 2240 & 2240 & 67 & 220 & -20 \\ 8 & 15 & 469 & -7091 & -72 & -72 & 18 & 7 & -103 & -103 & 333 & 22 & 133 \\ 9 & 15 & -7 & 103 & -72 & 133 & 18 & 333 & -103 & 7092 & -469 & 22 & -72 \\ 10 & 15 & -333 & 103 & 133 & -72 & 18 & -469 & 7091 & -103 & 7 & 22 & -72\end{array}$

EXPANSICNS IN HYARIO BASIS

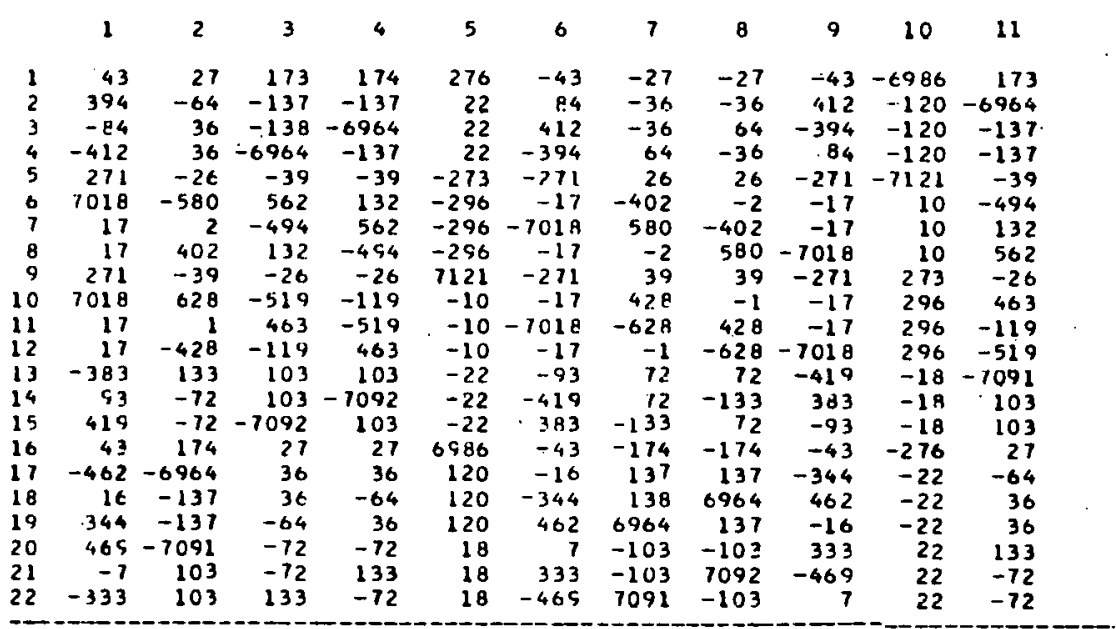


TABLE O. GECMETRY AND LCAO-MO'S FCR 1.4-PENTAOIYNE

\section{GECNETRY}

ATCN NUMBER EI,EMENT

$\begin{array}{ll}1 & H \\ 2 & C \\ 3 & C \\ 4 & C \\ 5 & C \\ 6 & C \\ 7 & H \\ 8 & H \\ 9 & \end{array}$

ix

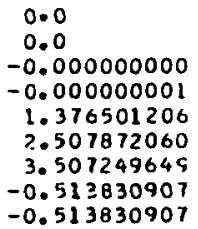

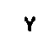

0.0

0.0

0.0
0.0

0.0

0.000000000

0.000000000

$-0.889981290$
0.0

1.060000000 2.260000000 3.720000000

4.206666652

4.606666639

4.959999961

4.083333322

4.083333322

EXPANSICNS IN SLATER BASIS

\begin{tabular}{|c|c|c|c|c|c|c|c|c|c|c|c|c|c|}
\hline & & 1 & 2 & 3 & 4 & 5 & 6 & 7 & 8 & 9 & 10 & 11 & 12 \\
\hline 1 & 15 & 33 & -19 & $\in 8 \in 7$ & 18 & -35 & -52 & -350 & -33 & 35 & 5 & -32 & 24 \\
\hline 2 & 25 & 3229 & & 6 & -33 & 95 & 94 & 601 & 32 & -95 & -9 & 97 & -3263 \\
\hline 2 & $P X$ & 2899 & -89 & 19 & $8 t$ & 377 & -205 & -6 & & -377 & 588 & -678 & 5844 \\
\hline 2 & PY & -5008 & 48 & 0 & 49 & 598 & 0 & 0 & -49 & 598 & 0 & 0 & 24 \\
\hline 2 & PZ & -2460 & 14 & -5343 & -13 & 39 & 38 & 80 & 2460 & -39 & -3 & 41 & -2455 \\
\hline 3 & 25 & $\begin{array}{l}-3213 \\
-2816\end{array}$ & $\begin{array}{r}-107 \\
79\end{array}$ & 14 & 102 & -50 & -292 & -4762 & 32 & 5 & 14 & -5 & 80 \\
\hline 3 & $p x$ & -2816 & 79 & 8 & -75 & -406 & 201 & 98 & 28 & 40 & -44 & 777 & $?$ \\
\hline 3 & PY & -4970 & -3 & 0 & -3 & -632 & 0 & 0 & -4946 & -631 & 0 & 0 & 23 \\
\hline 3 & PZ & 2544 & -72 & 193 & 69 & 112 & -205 & -5228 & -2544 & -112 & 7 & 196 & $25 \in S$ \\
\hline 4 & 25 & 46 & -46 & 168 & 46 & 339 & -18 & & -46 & -3392 & -168 & 3566 & $-1 E$ \\
\hline 4 & $P X$ & -201 & 14 & -9 & -145 & -3013 & 114 & 227 & 203 & 3013 & -207 & 5548 & 372 \\
\hline & PY & -473 & -46 & & 474 & -477 & & & -470 & -4777 & 0 & 0 & 2 \\
\hline 4 & P2. & 84 & -166 & -223 & 159 & 2.130 & -354 & 5965 & -84 & -2121 & -83 & 2203 & 10 \\
\hline 5 & 25 & 104 & 3214 & -14 & -3213 & -50 & -3180 & 5 & -106 & 51 & 614 & 4762 & -292 \\
\hline 5 & $P X$ & -91 & 3362 & 21 & -3327 & -241 & -526 & -74 & 93 & 24 & 179 & .4961 & 260 \\
\hline 5 & PY & -3 & -4927 & & -4987 & -632 & 59 & 0 & -3 & -63 & 0 & 0 & 0 \\
\hline 5 & D 2 & 49 & -1875 & -39 & $177 \mathrm{~B}$ & 345 & -6190 & 798 & -50 & -345 & 72 & -1649 & -121 \\
\hline$t$ & 25 & -34 & 3229 & 9 & -3229 & 95 & -3263 & -97 & 34 & -95 & -4876 & -601 & ५4 \\
\hline 6 & $P X$ & 42 & -1328 & -23 & $13 \in 3$ & $8 \mathbf{8}$ & 4262 & 265 & -42 & -88 & -5044 & 77 & -104 \\
\hline 6 & PY & 48 & -4965 & 0 & $-502 t$ & 598 & 60 & 0 & 48 & 598 & 0 & 0 & 0 \\
\hline 6 & PZ & -77 & -3623 & 53 & 352 & -369 & -4691 & -626 & 78 & 369 & 52 & 20 & 180 \\
\hline 7 & 15 & 18 & -33 & -5 & 33 & -35 & 24 & 32 & -18 & 35 & -6867 & 350 & -52 \\
\hline ด & 15 & -497 & -176 & -21 & -457 & 706 & 20 & -21 & -27 & 103 & 21 & 21 & 197 \\
\hline & 15 & 182 & 497 & 21 & 184 & -103 & 191 & -21 & 457 & $=7060$ & 21 & 21 & 154 \\
\hline
\end{tabular}

EXPANSICNS IN HYERIO EASIS

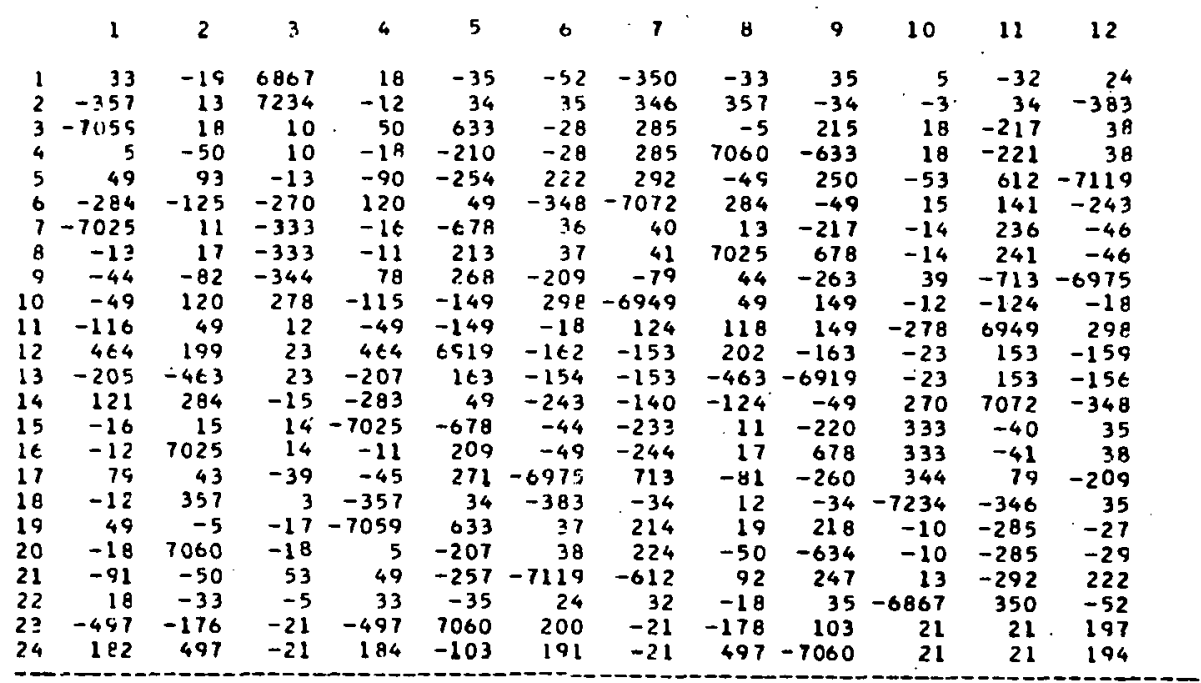


TABLE 7. GECMETRY ANO LCAO-MO'S FOR METHANE

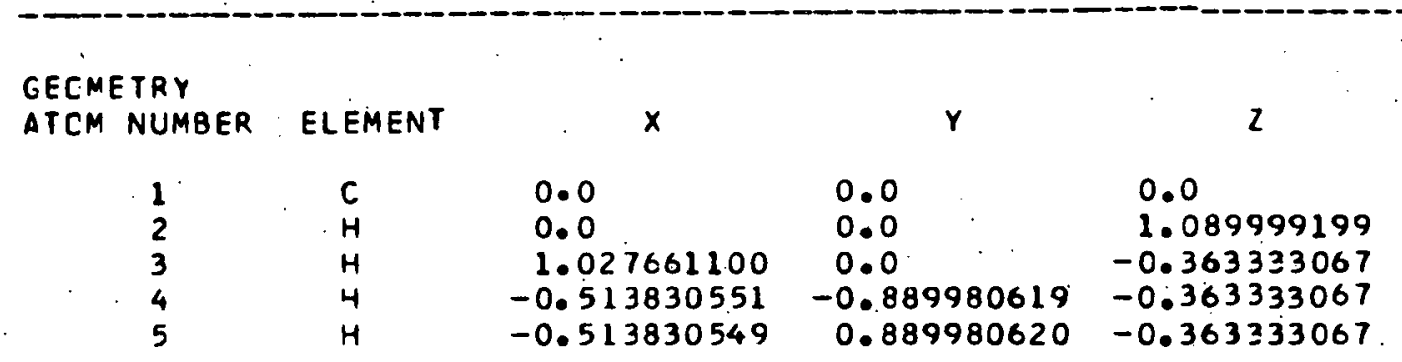

EXPANSICNS IN SLATER BASIS

$\begin{array}{rrrrrr} & & 1 & 2 & 3 & 4 \\ 1 & 2 S & 3671 & -3671 & 3671 & 3671 \\ 1 & P X & 0 & 2830 & -2830 & 5 \in t 1 \\ 1 & P Y & 0 & 4902 & 4902 & 0 \\ 1 & P Z & 6004 & 2001 & -2001 & -2001 \\ 2 & 1 S & 7101 & 104 & -104 & -104 \\ 3 & 1 S & -104 & 104 & -104 & 7101 \\ 4 & 1 S & -104 & -7101 & -104 & -104 \\ 5 & 1 S & -104 & 104 & 7101 & -104\end{array}$

EXPANSIONS IN HYBRID BASIS.

\begin{tabular}{rrrcrc} 
& 1 & 2 & 3 & 4 \\
1 & 7035 & -102 & 102 & 101 \\
2 & 102 & -102 & 102 & 7035 \\
3 & 102 & -7035 & 102 & 102 \\
4 & 102 & -102 & 7035 & 102 \\
5 & 7101 & 104 & -104 & -104 \\
6 & -104 & 104 & -104 & 7101 \\
7 & -104 & -7101 & -104 & -104 \\
8 & -104 & 104 & 7101 & -104 \\
\hline
\end{tabular}


TABLE 8. GECMETRY ANC LCAO-MO'S FOR ACETYLENE

GEOMETRY ATOM NUMBER

$\begin{array}{ll}1 & \text { C } \\ 2 & \text { C } \\ 3 & H \\ 4 & H\end{array}$

$x$

$$
0.0
$$$$
0.0
$$$$
-0.000000000
$$
$-0.000000000$
$Y$

$$
0.0
$$

0.0

0.0

0.0
0.0

1.199599809

$-1.059599466$

2.259999275

EXPANSIONS IN SLATER BASIS

$\begin{array}{rrrrrrr} & & 1 & 2 & 3 & 4 & 5 \\ 1 & 2 S & -3247 & -4874 & 3247 & 626 & -3247 \\ 1 & P X & -5769 & 0 & -3078 & 0 & 2690 \\ 1 & P Y & 223 & 0 & -4884 & 0 & -5108 \\ 1 & P Z & -2474 & 5334 & 2474 & 132 & -2474 \\ 2 & 2 S & -3247 & 636 & 3247 & -4874 & -3247 \\ 2 & P X & -5769 & 0 & -3078 & 0 & 2690 \\ 2 & P Y & 223 & 0 & -4884 & 0 & -5108 \\ 2 & P Z & 2474 & -132 & -2474 & -5334 & 2474 \\ 3 & 1 S & 32 & -6872 & -32 & -349 & 32 \\ 4 & 1 S & 32 & -348 & -32 & -6872 & 32\end{array}$

\section{EXPANSICNS IN HYBRID EASIS}

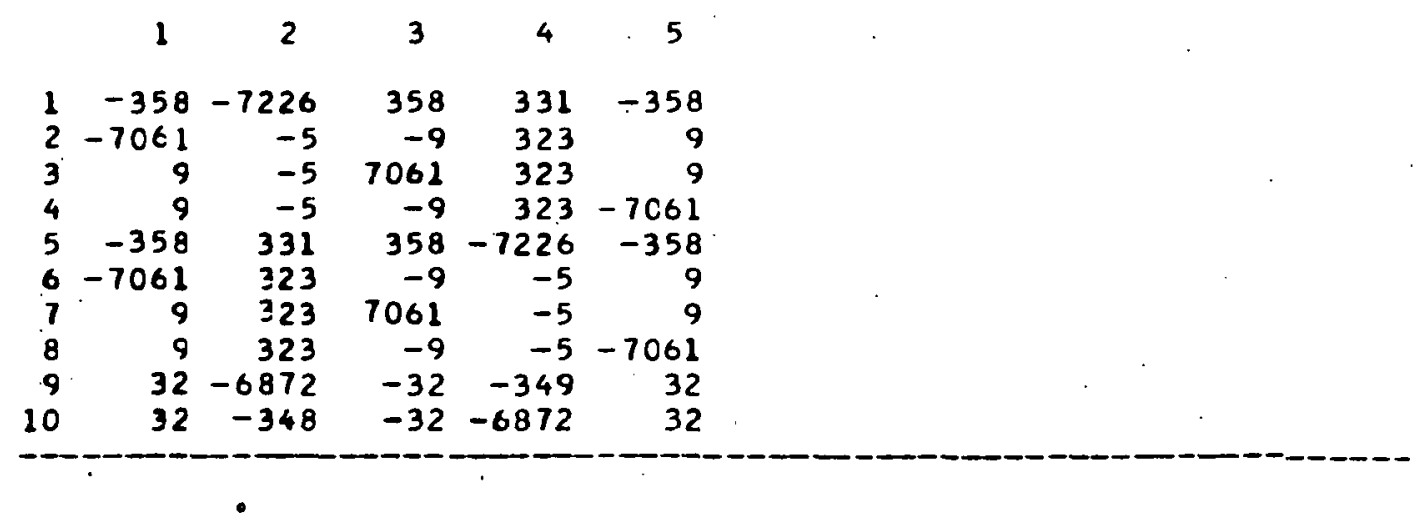


TABLE S. GEOMETRY ANO LCAO-MO'S FOR S-GAUCHE-1-BUTENE

\begin{tabular}{|c|c|c|c|c|}
\hline $\begin{array}{l}\text { CMETRY } \\
\text { ICP NUPBER }\end{array}$ & ELEMENT & $x$ & $Y$ & 2 \\
\hline $\begin{array}{r}1 \\
2 \\
3 \\
4 \\
5 \\
6 \\
7 \\
8 \\
9 \\
10 \\
11 \\
12\end{array}$ & $\begin{array}{l}C \\
C \\
C \\
C \\
H \\
H \\
H \\
H \\
H \\
\dot{M} \\
H \\
H\end{array}$ & $\begin{array}{r}0.0 \\
0.0 \\
-1.316358184 \\
-1.397936407 \\
0.935307370 \\
-0.935307371 \\
0.935307371 \\
-2.144844400 \\
-1.374098576 \\
-2.341903404 \\
-1.340196015 \\
-0.569450192\end{array}$ & $\begin{array}{l}0.0 \\
0.0 \\
-0.000000001 \\
-1.257404703 \\
0.0 \\
0.0 \\
0.000000000 \\
-0.000000001 \\
0.889980619 \\
-1.257404704 \\
-2.147385323 \\
-1.257404702\end{array}$ & $\begin{array}{l}0.0 \\
1.339999199 \\
2.099998951 \\
2.985367965 \\
-0.539999962 \\
-0.539999962 \\
1.879999161 \\
1.391684866 \\
2.726655793 \\
3.530367564 \\
2.358711123 \\
3.693682050\end{array}$ \\
\hline
\end{tabular}

EXPANSIONS IN SLATER BASIS

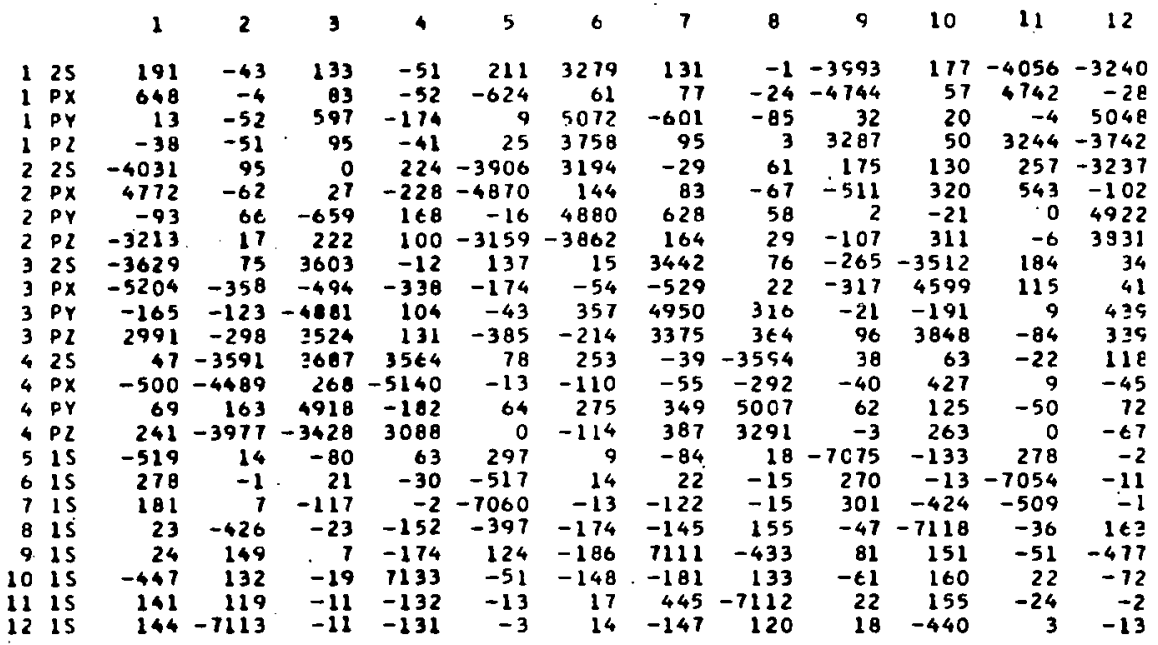

EXPARSICNS IN MYBRID EASIS

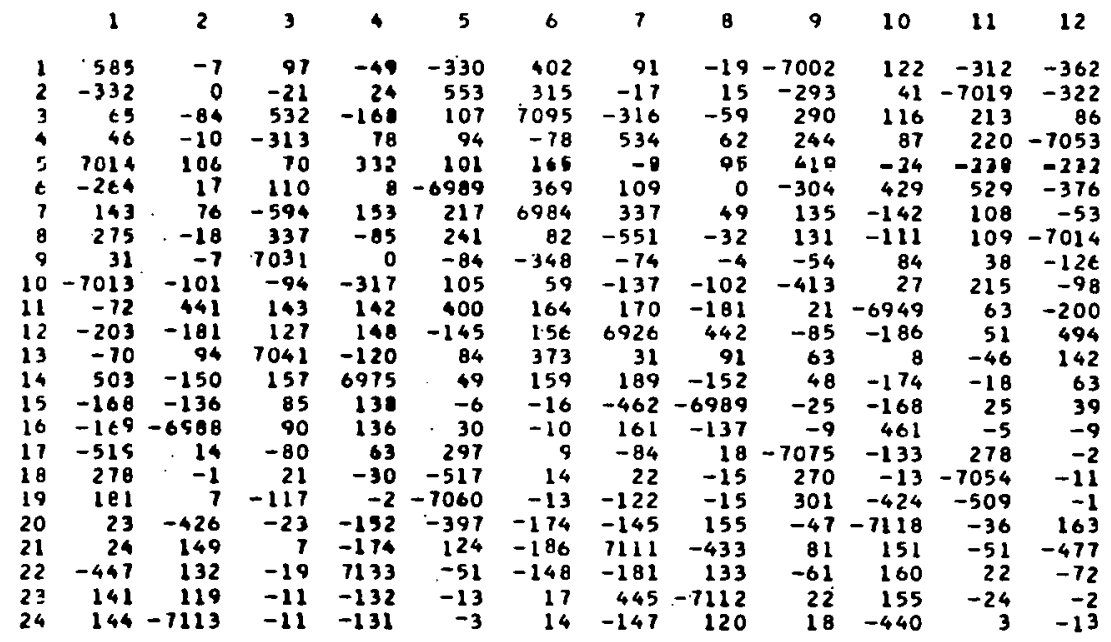


TABLE 10. GECMETRY ONC LCAO-MO'S FOR S-CIS-, S-IRANS-1, 4-PENTAOIENE

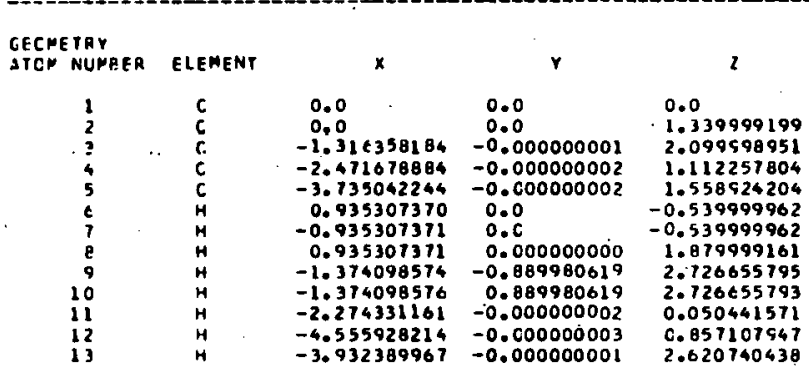

EXPANSICNS IN SLATER BASIS

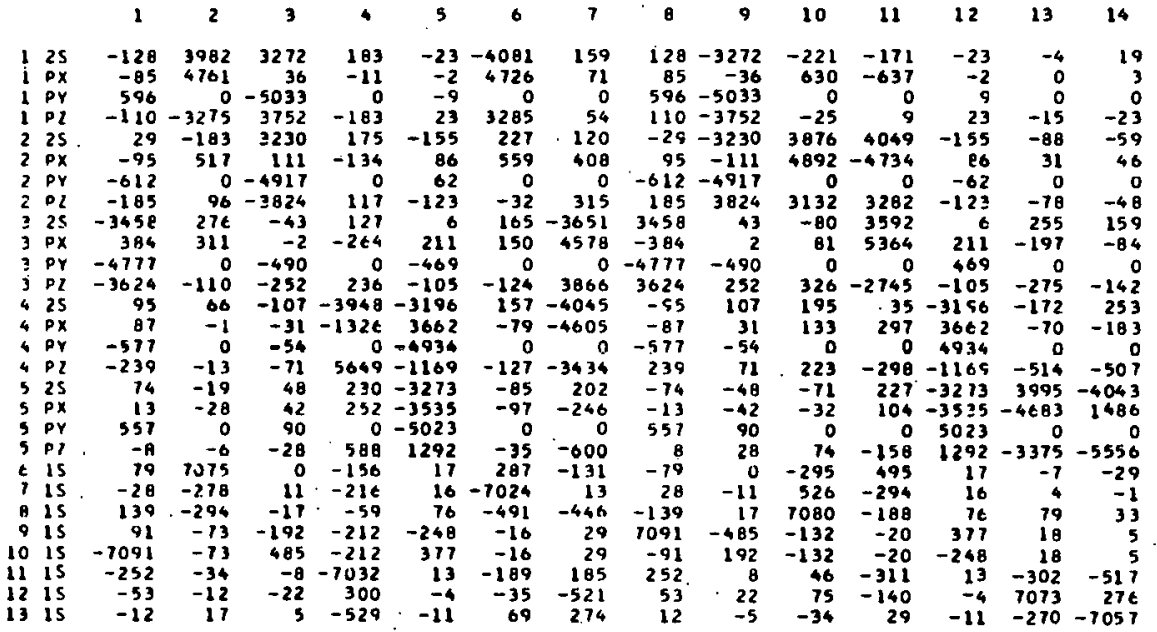

EXPANSICNS IN HYBRIO EASIS

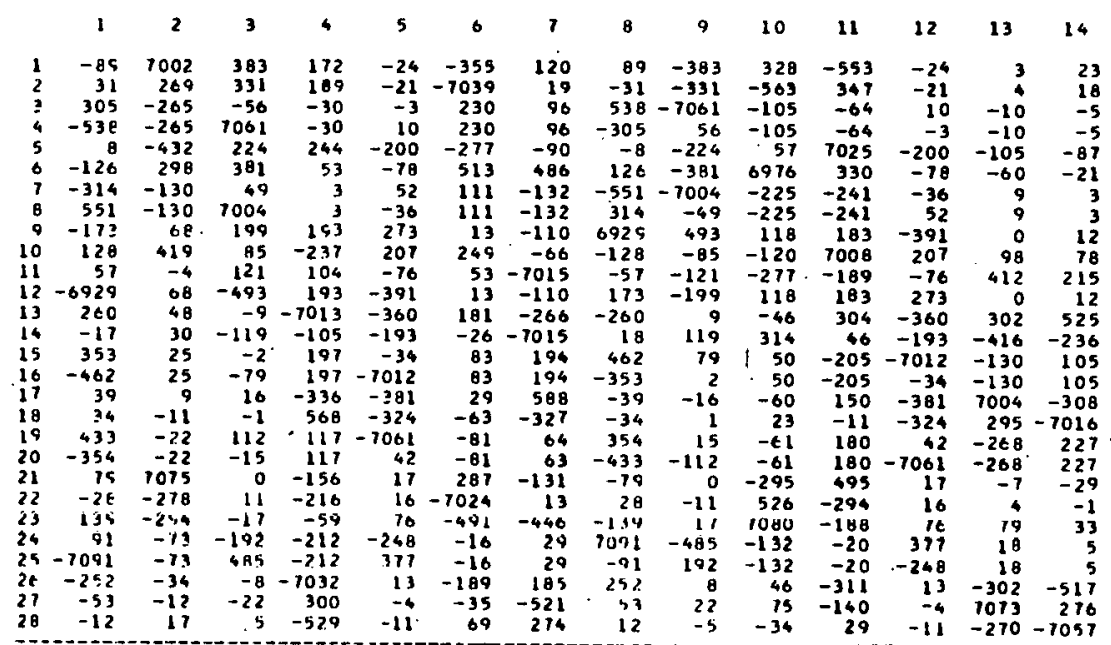


TABLE 11. GECMETRY ONC LCAOMMO'S FOR PENTENE

\begin{tabular}{|c|c|c|c|c|}
\hline $\begin{array}{l}\text { CECMETAY } \\
\text { ATOW RLPBER }\end{array}$ & ELENENT' & $x$ & $\gamma$ & 2 \\
\hline $\begin{array}{l}1 \\
2 \\
3 \\
4 \\
3 \\
0 \\
7 \\
8 \\
9 \\
10 \\
11 \\
12 \\
13 \\
14 \\
15\end{array}$ & $\begin{array}{l}C \\
C \\
C \\
C \\
C \\
H \\
H \\
H \\
H \\
H \\
H \\
H \\
H \\
H \\
H\end{array}$ & $\begin{array}{l}0.0 \\
0.0 \\
-1.316358184 \\
-2.48680825 \\
-3.820559913 \\
0.935307370 \\
-0.935307371 \\
0.935307371 \\
-1.374098574 \\
-1.374098576 \\
-2.429140435 \\
-2.429140434 \\
-3.878300303 \\
-3.878300305 \\
-4.649046129\end{array}$ & $\begin{aligned} 0.0 \\
0.0 \\
-0.000000001 \\
-0.000000002 \\
-0.000000002 \\
0.0 \\
0.0 \\
0.000000000 \\
-0.889980619 \\
0.88990619 \\
0.889980617 \\
-0.88990621 \\
-0.889980620 \\
0.88990619 \\
-0.000000003\end{aligned}$ & $\begin{array}{r}0.0 \\
1.339999199 \\
2.099998951 \\
1.099260909 \\
1.869260990 \\
-0.539999962 \\
-0.53999962 \\
1.879999161 \\
2.72665795 \\
2.726655793 \\
0.472604065 \\
0.472604066 \\
2.495517734 \\
2.495917731 \\
1.160546804\end{array}$ \\
\hline
\end{tabular}

EXPANSICAS IN SLATER GASIS

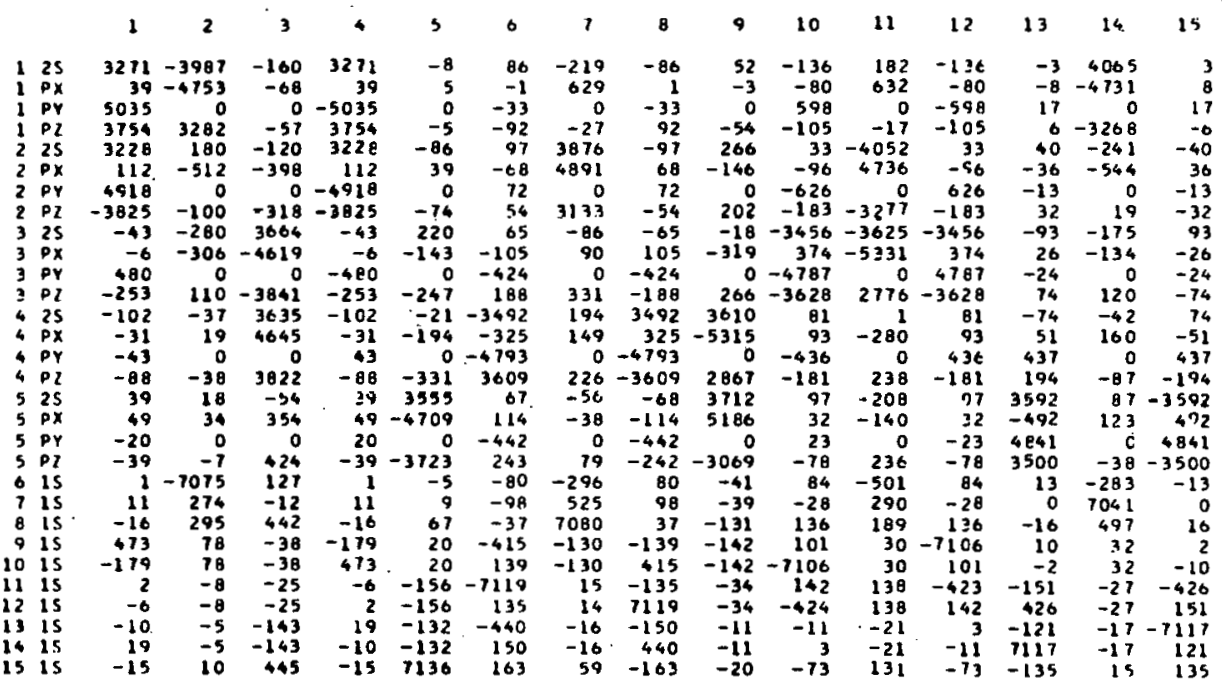

EXPARSICNS IN MYORIO EASIS

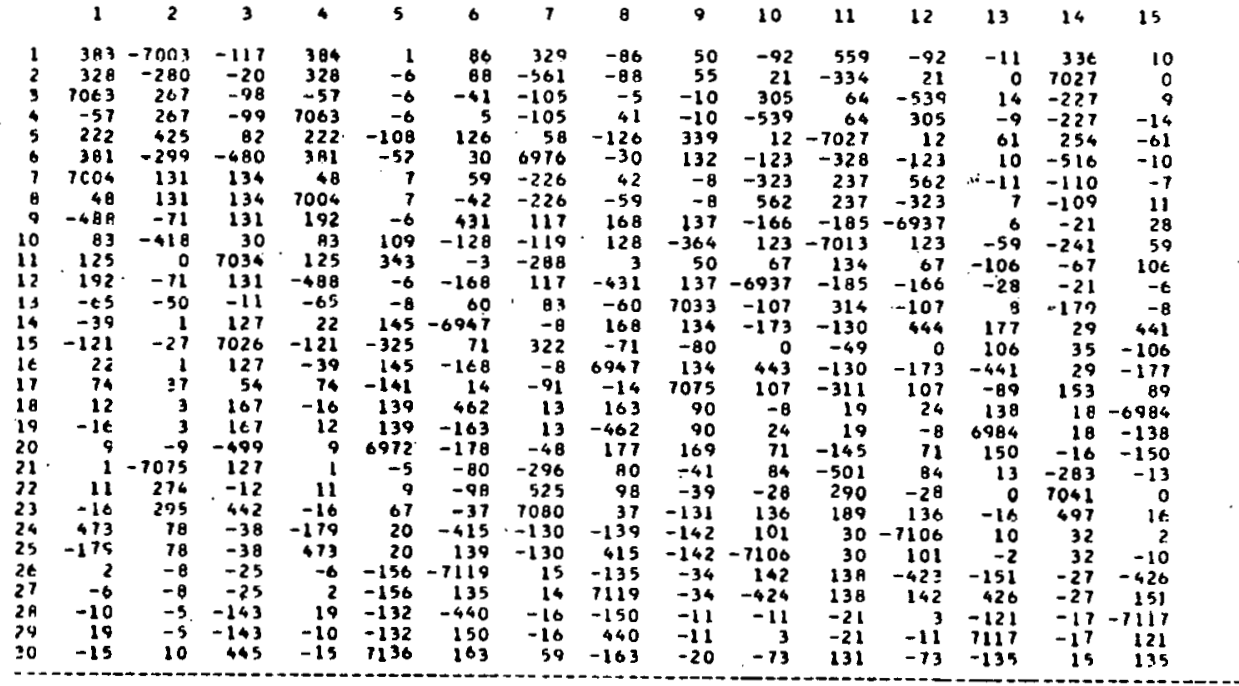


IDBLE 12. GECMETRY ANC ICAO-MOOS FCR VIAYL ETHYNYL ETHANE

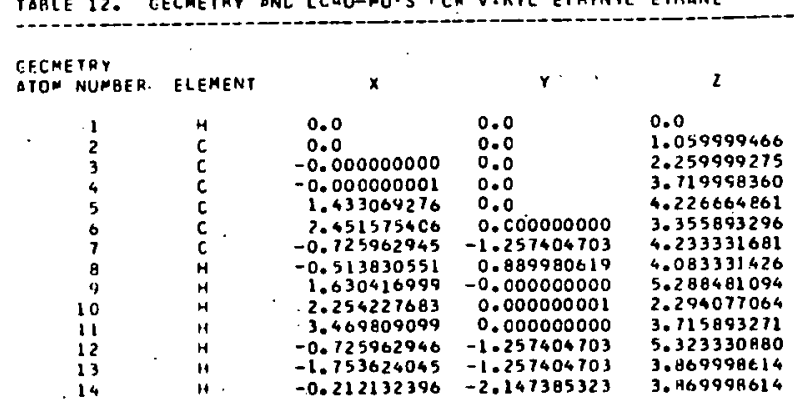

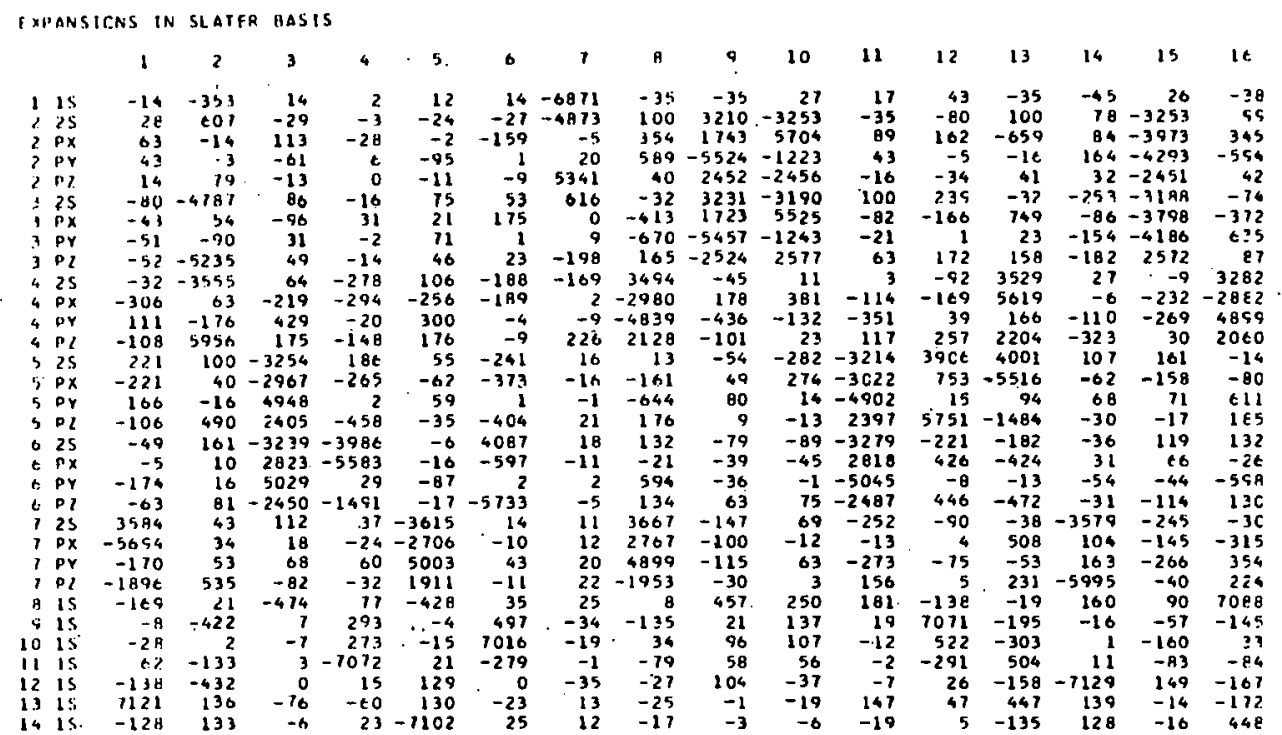

TXPANSICAS IN MYBRIO RASIS

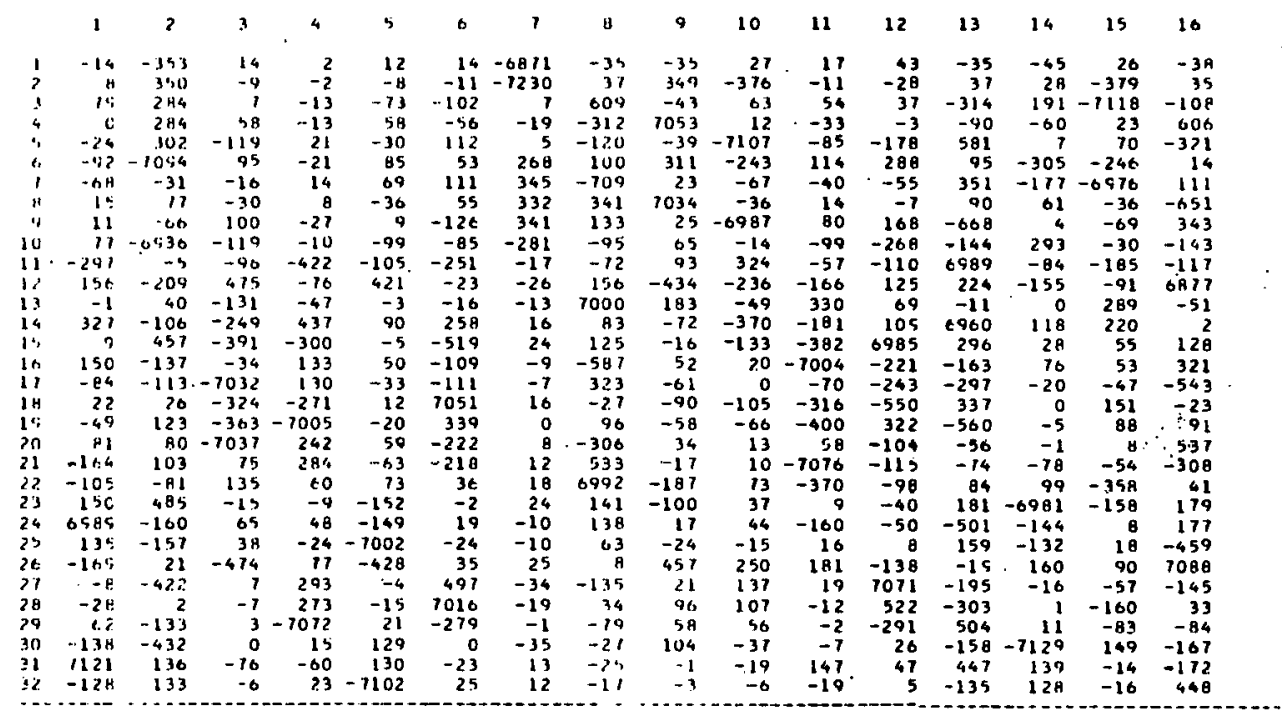


TAHLE 13. GECMETRY ANC LCAO-MO'S FOR VIAYL ETHYNYL METHANE

\begin{tabular}{|c|c|c|c|c|}
\hline $\begin{array}{l}\text { CECMETPY } \\
\text { ATOH NUYEER }\end{array}$ & ELEMENT & $x$ & $r$ & 2 \\
\hline $\begin{array}{l}1 \\
2\end{array}$ & $\stackrel{\mathrm{H} \cdot}{\mathrm{C}}$ & $\begin{array}{l}0.0 \\
0.0\end{array}$ & $\begin{array}{l}0.0 \\
0.0\end{array}$ & $\begin{array}{l}0.0 \\
1.059999466\end{array}$ \\
\hline 3 & $\mathrm{c}$ & -0.000000000 & 0.0 & 2.259599275 \\
\hline 4 & c & -0.000000001 & 0.0 & 3.719998360 \\
\hline s & $\mathrm{c}$ & 1.433069276 & 0.0 & 4.226064861 \\
\hline 6 & C & 2.451575406 & 0.000000 & 3.355893296 \\
\hline$i$ & H & -0.513030551 & 0.889980619 & 4.083331426 \\
\hline 8 & H & -0.513830551 & -0.889990619 & 4.083331426 \\
\hline 9 & H & 1.630416999 & -0.000000000 & 5.280481094 \\
\hline 10 & H & 2.254227683 & 0.000000001 & 2.294077064 \\
\hline il & H & 3.469809099 & 0.000000000 & 3.715093271 \\
\hline
\end{tabular}

EXPANSICNS IN SLATER BASIS

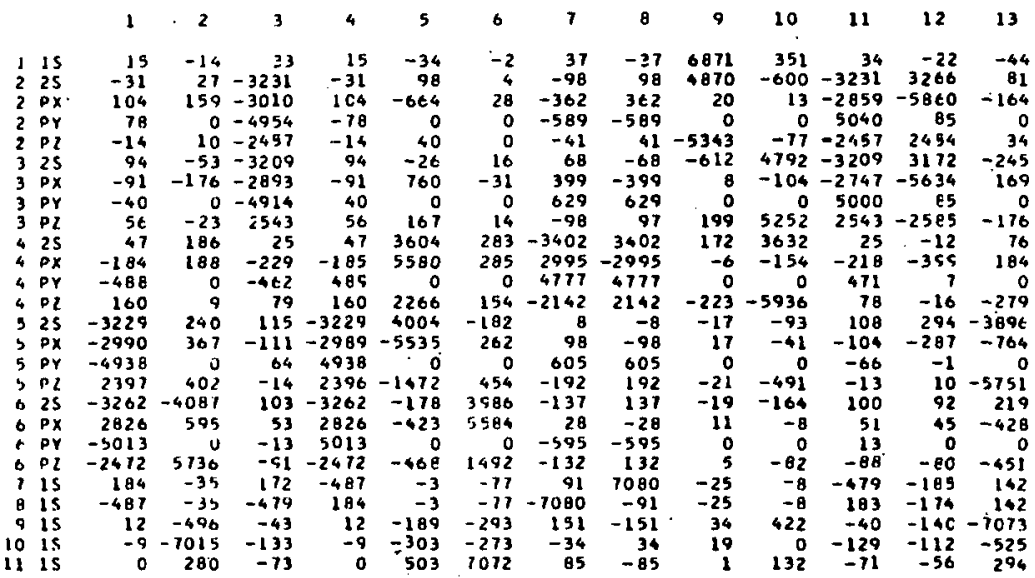

EXPANSICNS IN HYBRID EASIS

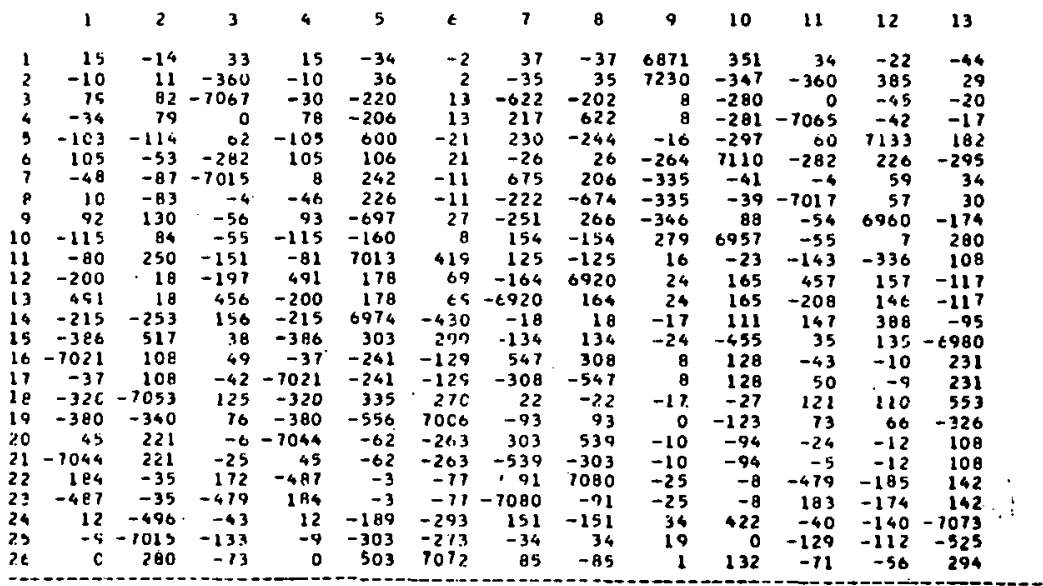




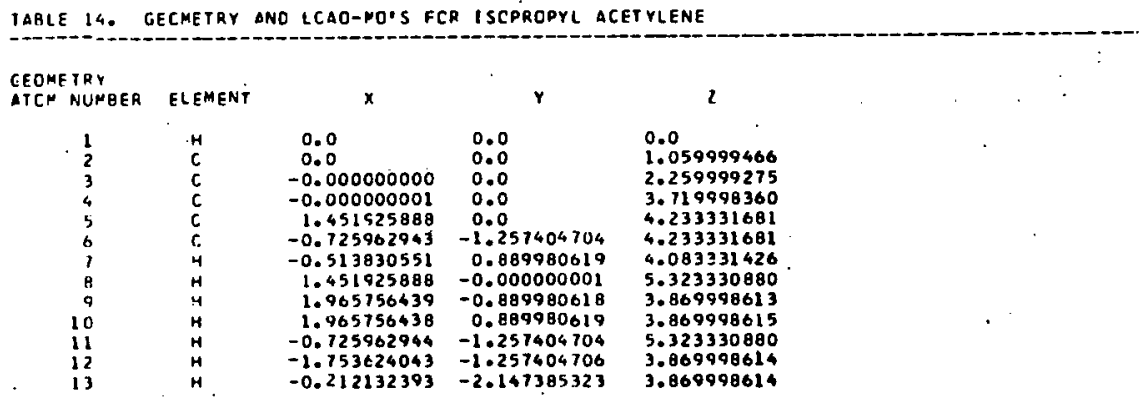

EXPANSICAS IN SLATER BASIS

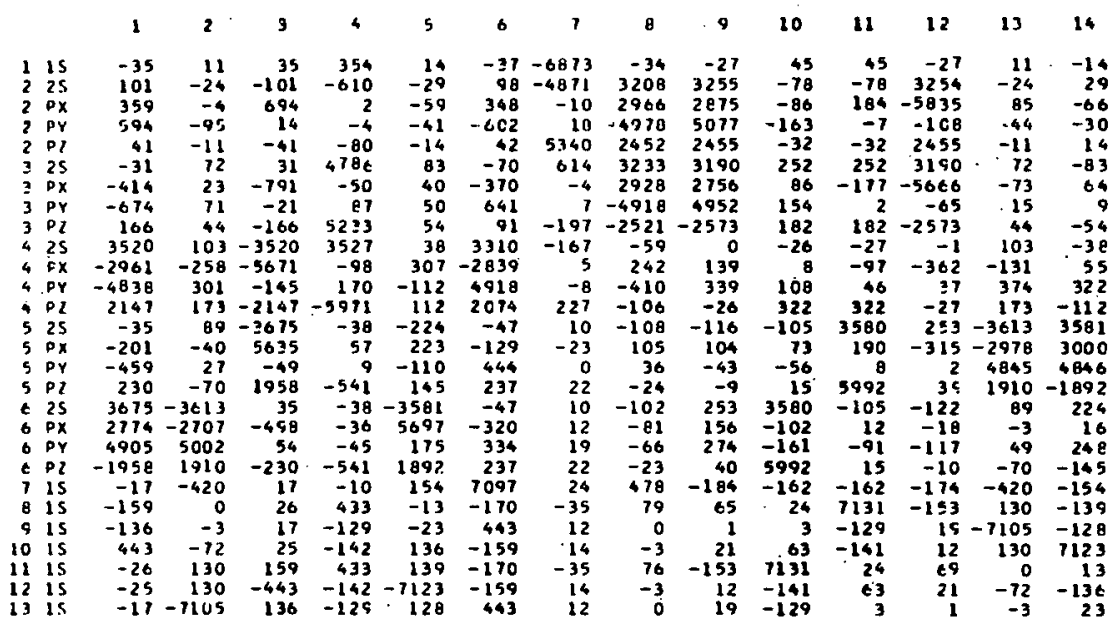

ExPANSICNS IN HYRAID gaSIS

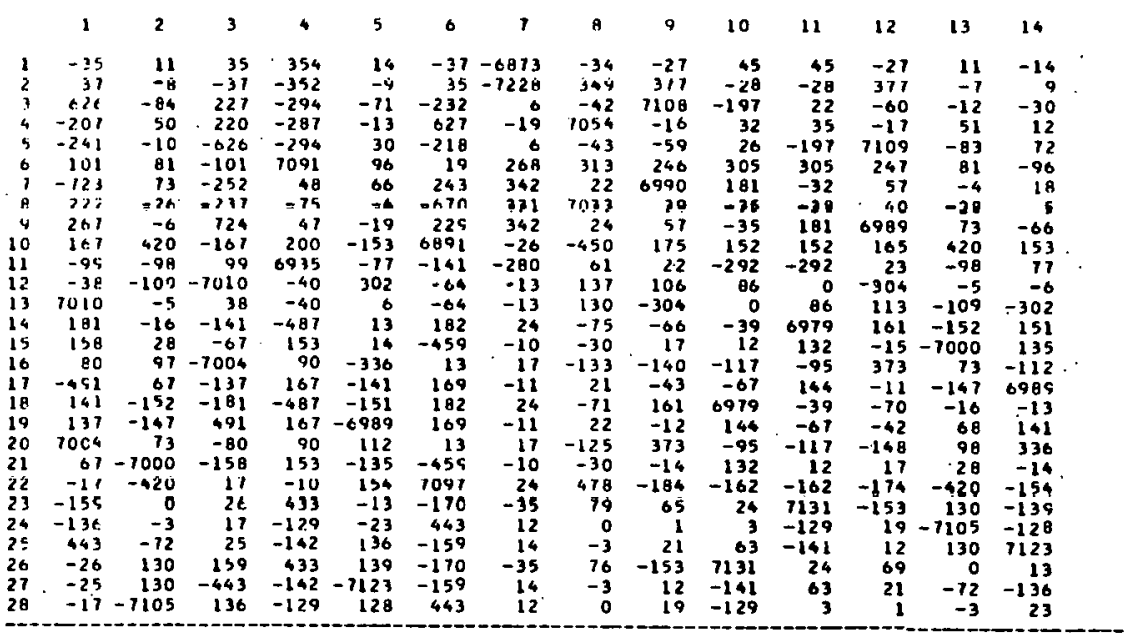


TABLE 15. geCMETRY ANC LCAO-MO'S for ETHANE

GEOMETRY

ATCN NUMBER ELEMENT

$x$

$\mathbf{Y}$

0.0

.0 .0

0.0

$-0.889980619$

0.889980620

$-0.000000000$

$-0.889980619$

0.889980619
0.0

1.53 .9599962

$-0.363333067$

$-0.363333067$

$-0.363333067$

1.903333028

$1.903333 \mathrm{C2B}$

1.903333028

EXPANSICAS IN SLATER EASIS

$\begin{array}{rrrrrrrrr}1 & & 1 & 2 & 3 & 4 & 5 & 6 & 7 \\ 1 & 2 S & -3577 & -54 & -3650 & -54 & -54 & 3577 & 3577 \\ 1 & P X & 2794 & 258 & 0 & -517 & 258 & -2794 & 5588 \\ 1 & P Y & 4839 & 448 & 0 & 0 & -448 & 4839 & 0 \\ 1 & P Z & 2190 & 40 & -6031 & 40 & 40 & -2190 & -2190 \\ 2 & 2 S & 54 & 3577 & -3690 & 3577 & 3577 & -54 & -54 \\ 2 & P X & 258 & 2794 & 0 & -5588 & 2794 & -258 & 517 \\ 2 & P Y & 448 & 4839 & 0 & 0 & -4839 & 448 & 0 \\ 2 & P 2 & 40 & 2190 & 6031 & 2190 & 2190 & -40 & -40 \\ 3 & 1 S & 123 & -158 & 5 & 440 & -158 & -123 & 7115 \\ 4 & 15 & -7115 & 440 & 5 & -158 & -158 & -123 & -123 \\ 5 & 15 & 123 & -158 & 5 & -158 & 440 & 7115 & -123 \\ 6 & 15 & 158 & -123 & 5 & 7115 & -123 & -158 & 440 \\ 7 & 15 & 158 & -123 & 5 & -123 & 7115 & 440 & -158 \\ 8 & 15 & -440 & 7115 & 5 & -123 & -123 & -158 & -158\end{array}$

EXPANSICAS IN HYBRID EASIS

\begin{tabular}{rrrrrrrr} 
& 1 & 2 & 3 & 4 & 5 & 6 & 7 \\
1 & 107 & 7 & -7068 & 7 & 7 & -107 & -107 \\
2 & -135 & 172 & -104 & -461 & 172 & 135 & 6983 \\
3 & -6983 & -461 & -104 & 172 & 172 & 139 & 139 \\
4 & -139 & 172 & -104 & 172 & -461 & 6983 & 139 \\
5 & -7 & -108 & -7068 & -107 & -107 & 7 & 7 \\
6 & -172 & 139 & -104 & 6983 & 139 & 172 & -461 \\
7 & -172 & 139 & -104 & 139 & -933 & -461 & 172 \\
8 & 461 & 6583 & -104 & 139 & 139 & 172 & 172 \\
9 & 123 & -158 & 5 & 440 & -158 & -123 & 1115 \\
10 & -7115 & 440 & 5 & -158 & -158 & -123 & -123 \\
11 & 123 & -158 & 5 & -158 & 440 & 715 & -123 \\
12 & 158 & -123 & 5 & 7115 & -123 & -158 & 440 \\
13 & 158 & -123 & 5 & -123 & 7115 & 440 & -158 \\
14 & -440 & 7115 & 5 & -123 & -123 & -158 & -158 \\
\hline
\end{tabular}


tahle 16. gecmetry ano lcaa-mo's for pentane

GeCMETRY

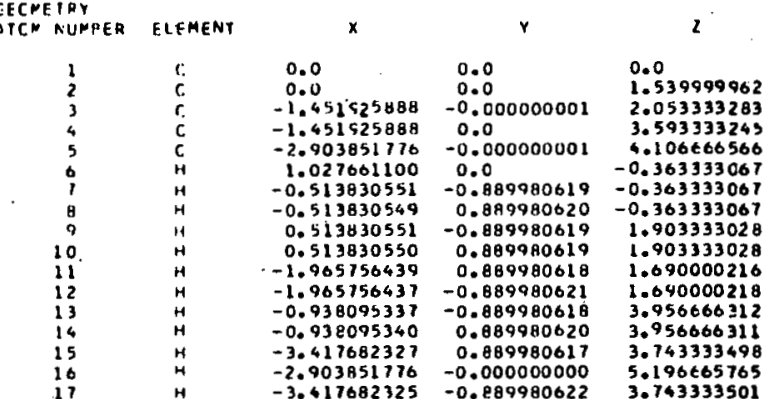

EXPANSICNS IN SLATER BASIS

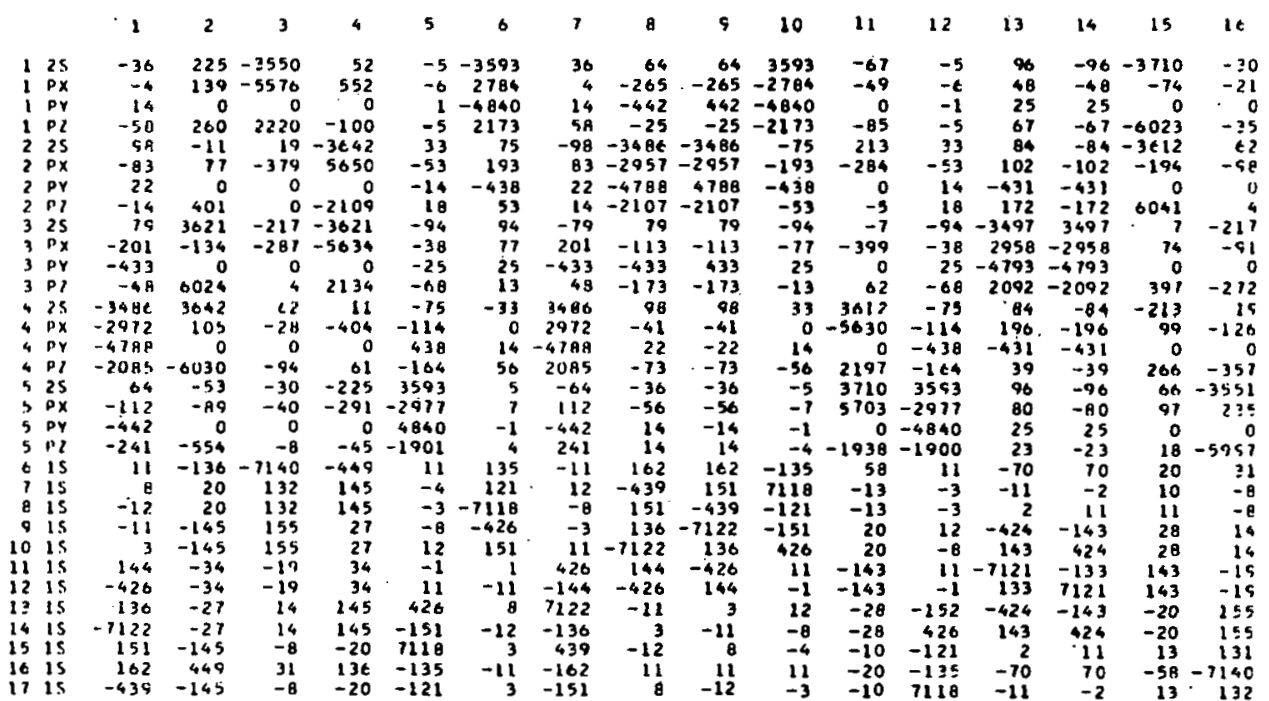

FXPANSICNS IN HYSRIO EASIS

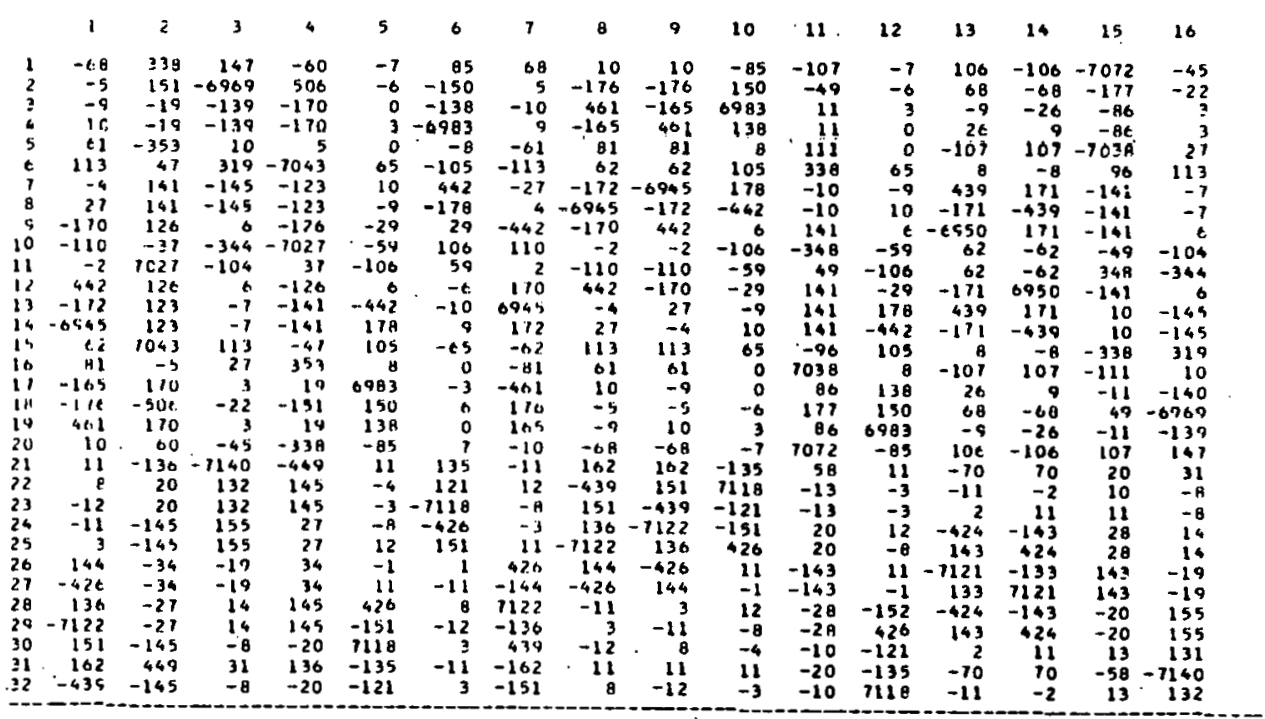


IARLE 17. GeCMETRY onC LCAO-MO'S FGR isceutane

GICNETAY

DIOM NLMBER ELEMENT

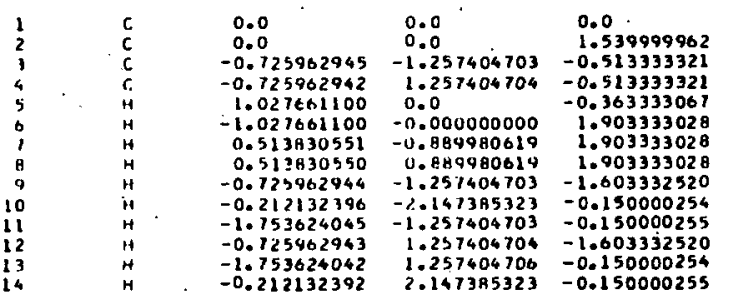

EXPANSICNS IN SLATER BASIS

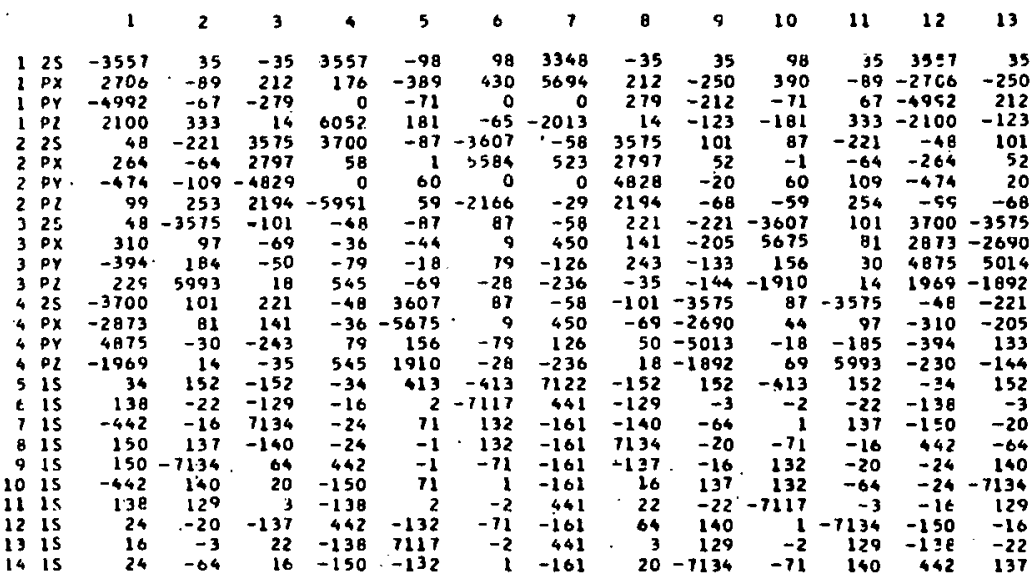

EXPANSICNS IN HYARIO AASIS

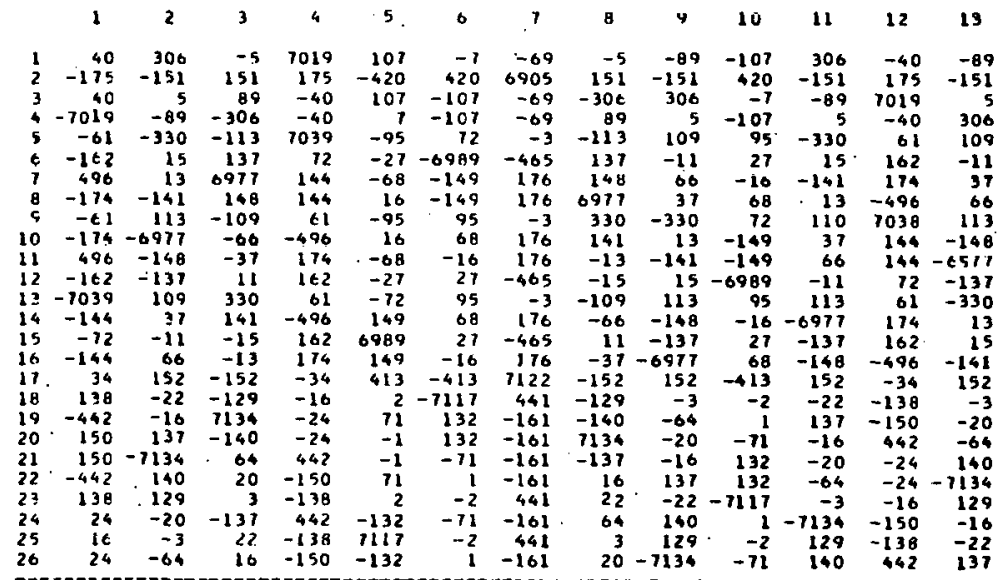


IABLE 18. GEOMEIAY AND LCAO-MOS FOR ISCPENTANE

\begin{tabular}{|c|c|c|c|c|}
\hline $\begin{array}{l}\text { GECMEIRY } \\
\text { ATCW NUMPER }\end{array}$ & ELEMENT & $x$ & r & 2 \\
\hline 1 & c & $\begin{array}{l}0.0 \\
0.0\end{array}$ & 0.0 & 0.0 \\
\hline 2 & c & $\begin{array}{l}0.0 \\
-0.125962945\end{array}$ & $\begin{array}{c}0.0 \\
-1.257404703\end{array}$ & $\begin{array}{r}1.539999962 \\
-0.51333332\end{array}$ \\
\hline $\begin{array}{l}3 \\
4\end{array}$ & $c$ & $\begin{array}{l}-0.725962945 \\
-0.125962942\end{array}$ & 1.257404704 & $\begin{array}{l}-0.513333321 \\
-0.513333321\end{array}$ \\
\hline $\begin{array}{l}4 \\
5\end{array}$ & c & 0.725962945 & -1.257406703 & 2.053333283 \\
\hline t & 4 & $1.027 \leq 61100$ & 0,0 & -0.363333067 \\
\hline 1 & H & -1.027661100 & -0.0000000 & 1.903333028 \\
\hline e & H & 0.513830550 & 0.08998 & .90333 \\
\hline 9 & H & -0.725562944 & -1.257404703 & -1.603332520 \\
\hline 10 & H & -0.212132396 & -2.147385323 & -0.150000254 \\
\hline ii & $H$ & -1.153624045 & -1.257404703 & $-c .150000255$ \\
\hline 12 & 4 & -0.725962943 & 1.257404704 & -1.60 \\
\hline 13 & H & -1.753624042 & 1.257404706 & -0.150000254 \\
\hline 16 & M & -0.212132392 & 2.147385323 & -0.150000255 \\
\hline 15 & $H$ & 0.725962 .945 & -1.257404703 & 3.143332482 \\
\hline 16 & N & 0.212132397 & $.1+7385323$ & 1.690000216 \\
\hline & & $1.75 ? 624045$ & 2.257404702 & 1.690000217 \\
\hline
\end{tabular}

EXPANSICAS IN SLATER BaSIS

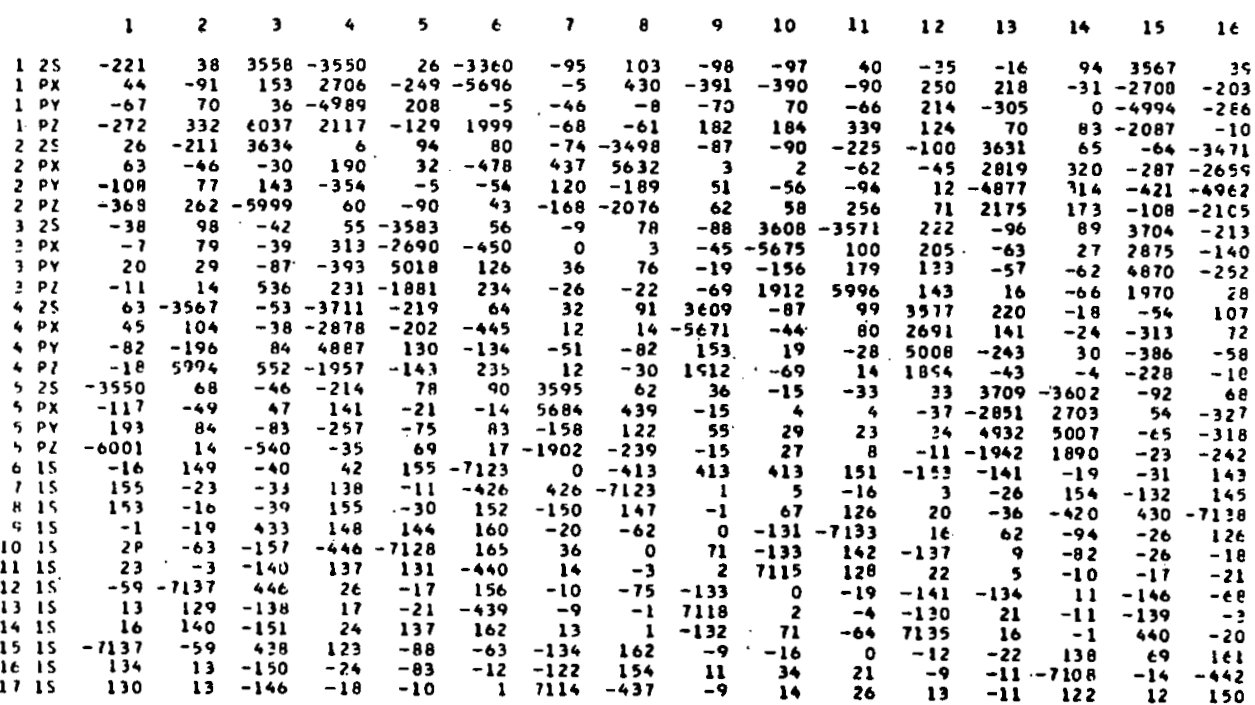

EXPANSTENS IN HYBRIO EASIS

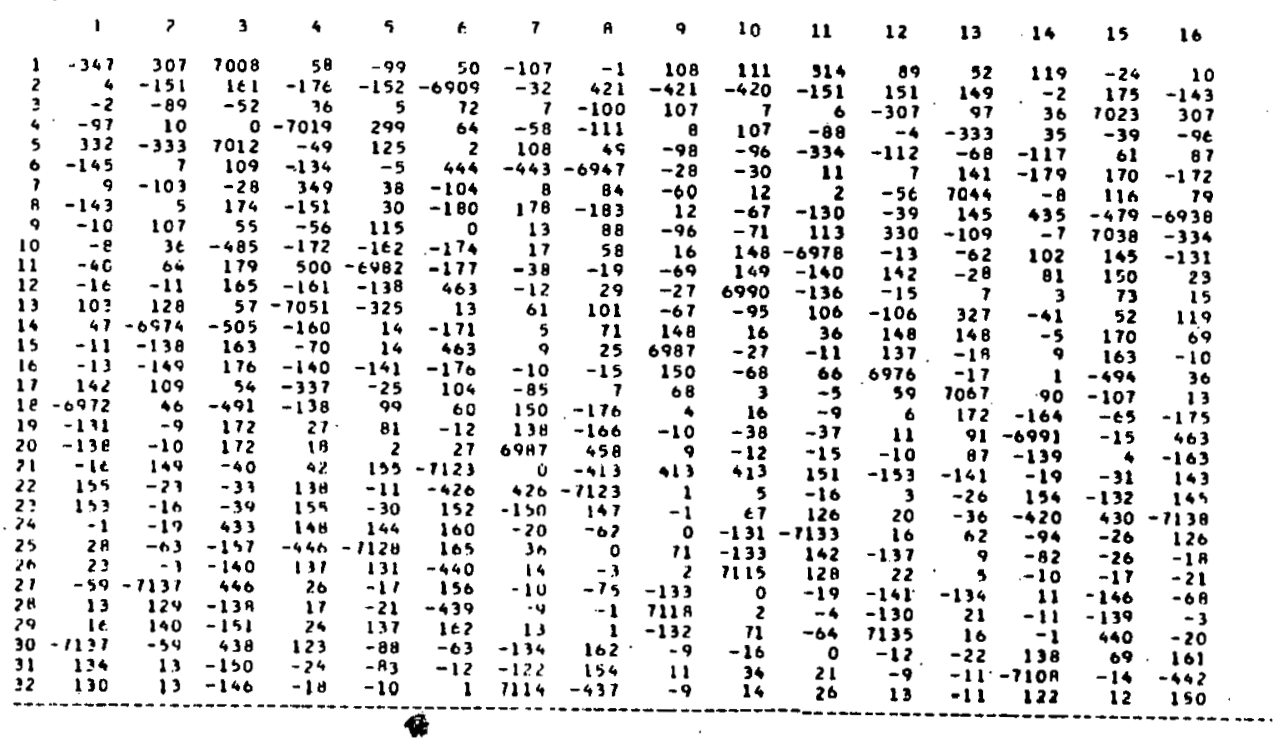




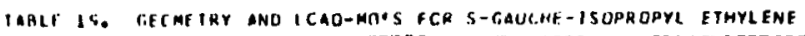

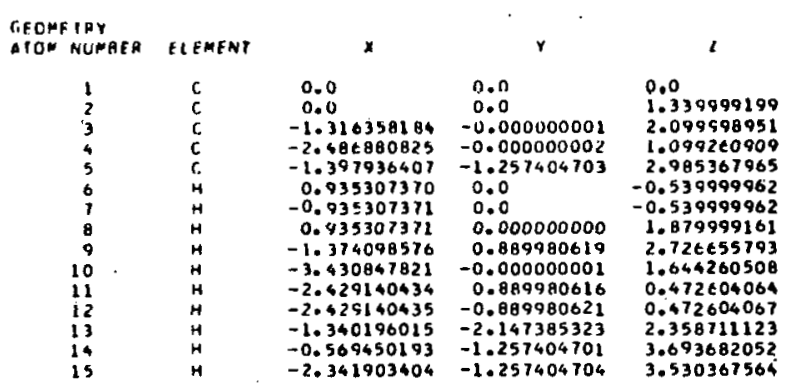

EXPANSICNS IN SLATER BASIS

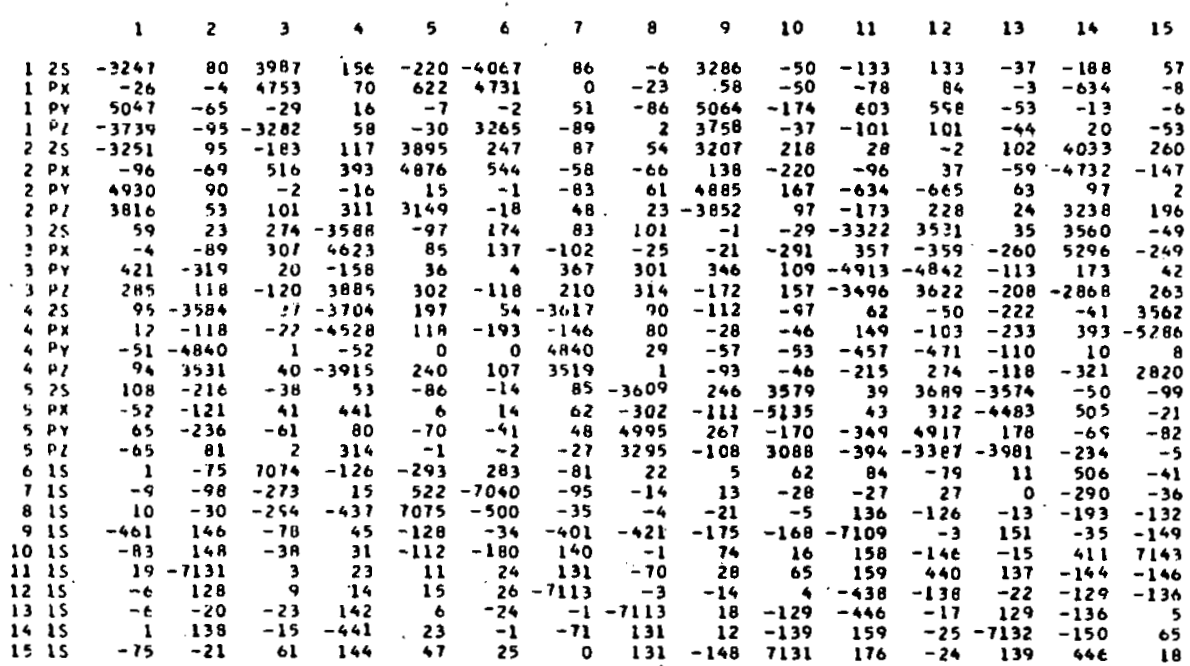

EXPANSICAS IN HYORIO RASIS

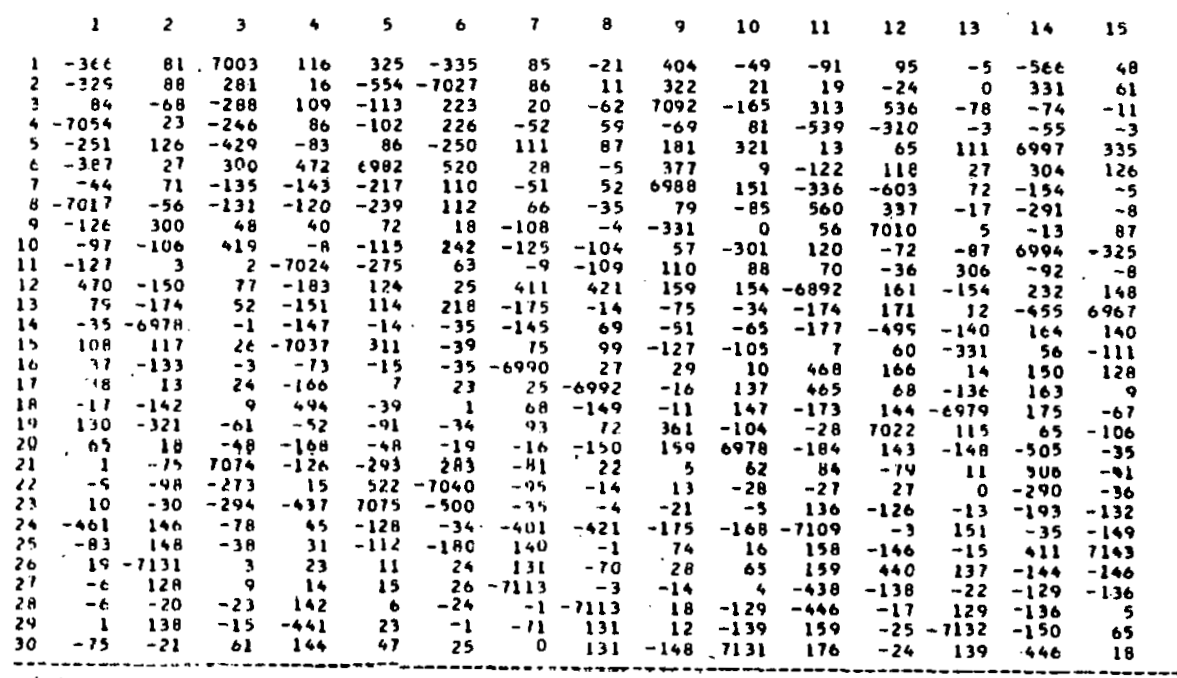


TABLE 20. GECMETRY ANC LCAD-MO'S FOR ETHYLENE

GEOMETRY

ATCM NUNEER

$\begin{array}{ll}1 & C \\ 2 & C \\ 2 & H \\ 4 & H \\ 5 & H \\ 6 & H\end{array}$

ELEMENT

$C$
$C$
$H$
$H$
$H$
$H$

$x$

0.0
0.0
0.935307370
-0.935307371
-0.935307370
0.935307371

\section{$Y$}

0.0
0.0
$0 . \mathrm{C}$
0.0
-0.000000000
0.000000000

0.0

1.339599199 $-0.539999962$ $-0.539999962$

1.879999161

1.879999161

EXPANSICNS IN SLATER BASIS

$\begin{array}{rrrrrrrr} & & 1 & 2 & 3 & 4 & 5 & 6 \\ 1 & 2 S & 214 & -4019 & -3264 & -3264 & 214 & -4019 \\ 1 & P X & 627 & -4757 & 0 & 0 & -627 & 4757 \\ 1 & P Y & 0 & 0 & 4999 & -5000 & 0 & 0 \\ 1 & P 2 & 36 & 3270 & -3787 & -3787 & 36 & 3270 \\ 2 & 2 S & -4019 & 214 & -3264 & -3244 & -4019 & 214 \\ 2 & P X & 4757 & -627 & 0 & 0 & -4757 & 627 \\ 2 & P Y & 0 & 0 & 4999 & -5000 & 0 & 0 \\ 2 & P 2 & -3270 & -36 & 3787 & 3787 & -3270 & -36 \\ 3 & 15 & -518 & -7045 & -14 & -14 & 299 & 253 \\ 4 & 1 S & 299 & 253 & -14 & -14 & -518 & -7 C 45 \\ 5 & 15 & -7045 & -518 & -14 & -14 & 253 & 299 \\ t & \text { IS } & 253 & 299 & -14 & -14 & -7045 & -518\end{array}$

EXPANSICNS IN HYBRID BASIS

\begin{tabular}{rrrrrrr} 
& 1 & 2 & 3 & 4 & 5 & 6 \\
1 & 552 & -7020 & -338 & -338 & -334 & -252 \\
2 & -334 & -292 & -338 & -338 & 552 & -7020 \\
3 & 108 & 247 & 16 & -7054 & 108 & 247 \\
4 & 108 & 247 & -7054 & 16 & 108 & 247 \\
5 & -7020 & 552 & -338 & -338 & -292 & -334 \\
6 & -292 & -334 & -338 & -338 & -7020 & 552 \\
7 & 247 & 108 & 16 & -7054 & 247 & 108 \\
8 & 247 & 108 & -7054 & 16 & 247 & 108 \\
9 & -518 & -7045 & -14 & -14 & 299 & 253 \\
10 & 299 & 253 & -14 & -14 & -518 & -7045 \\
11 & -7045 & -518 & -14 & -14 & 253 & 299 \\
12 & 253 & 299 & -14 & -14 & -1045 & -518 \\
\hline
\end{tabular}


TABLE 21. GECMETRY ANC LCAO-MO'S FCR PRCPENE.

GECPETRY

ELEMENT

$x$

0.0

0.0

$-1.316358184$

0.935307370

$-0.935307371$

0.935307371

$-1.374098574$

$-2.144844400$

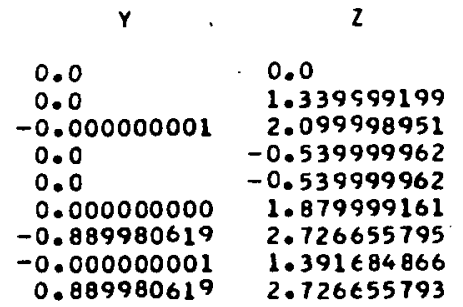

EXPANSICNS IN SLATER BASIS

$\begin{array}{rrrrrrrrrrr} & & 1 & 2 & 3 & 4 & 5 & 6 & 7 & 9 & 9 \\ 1 & 2 S & 137 & 3995 & -3261 & -181 & 208 & -4055 & 187 & -137 & -3261 \\ 1 & P X & 77 & 4745 & -43 & -57 & -629 & 4743 & 643 & -77 & -43 \\ 1 & P Y & -600 & 0 & -5037 & 0 & 0 & 0 & 0 & -600 & 5037 \\ 1 & P Z & 97 & -3286 & -3756 & -52 & 22 & 3247 & -39 & -57 & -3756 \\ 2 & 2 S & -24 & -171 & -3211 & -124 & -3895 & 256 & -4035 & 23 & -3211 \\ 2 & P X & 79 & 507 & -121 & -325 & -4879 & 539 & 4773 & -79 & -121 \\ 2 & P Y & 079 & 0 & 0 \\ 2 & P Y & 626 & 0 & -4913 & 0 & 0 & 0 & 0 & 626 & 4913 \\ 2 & P Z & 182 & 106 & 3840 & -312 & -3150 & -4 & -3237 & -182 & 3840 \\ 3 & 25 & 3547 & 271 & 23 & 3619 & 121 & 184 & -3694 & -3547 & 23 \\ 3 & P X & -531 & 318 & 48 & -4654 & -182 & 114 & -5242 & 531 & 49 \\ 3 & P Y & 4835 & 0 & -495 & 0 & 0 & 0 & 0 & 4835 & 495 \\ 3 & P Z & 3525 & -84 & 304 & -3764 & -418 & -80 & 2913 & -3525 & 304 \\ 4 & 15 & -86 & 7074 & -5 & 133 & 300 & 278 & -516 & 86 & -5 \\ 5 & 15 & 21 & -270 & -11 & 15 & -520 & -7053 & 278 & -21 & -11 \\ \epsilon & 15 & -129 & -302 & 3 & 425 & -7061 & -507 & 176 & 129 & 3 \\ 7 & 15 & -89 & -81 & -491 & -136 & 129 & -52 & 9 & -7102 & 189 \\ 8 & 15 & -126 & 49 & 172 & 7111 & -409 & -35 & 5 & 126 & 172 \\ 9 & 15 & 7102 & -81 & 189 & -136 & 129 & -52 & 8 & 89 & -491\end{array}$

EXPANSIONS IN MYBRIO BASIS

\begin{tabular}{rrrrrrrrrr} 
& 1 & 2 & 3 & 4 & 5 & 6 & 7 & 8 & 9 \\
1 & 94 & 7004 & -380 & -123 & -334 & -313 & 579 & -94 & -380 \\
2 & -15 & 292 & -319 & -43 & 556 & -7021 & -330 & 15 & -319 \\
3 & -312 & -266 & -7062 & -104 & 98 & 219 & 53 & -537 & 61 \\
4 & 537 & -266 & 61 & -104 & 98 & 219 & 53 & 312 & -7062 \\
5 & 4 & -414 & -200 & 30 & -84 & -235 & -7027 & -4 & -200 \\
6 & 117 & 303 & -372 & -429 & -6985 & 527 & -275 & -117 & -372 \\
7 & 327 & -131 & -7003 & 130 & 228 & 106 & 222 & 558 & -53 \\
8 & -558 & -131 & -53 & 130 & 228 & 106 & 221 & -327 & -7003 \\
9 & 134 & 79 & 511 & 148 & -139 & 46 & -156 & -6972 & -189 \\
10 & -151 & 410 & -83 & -50 & 104 & 213 & -7040 & 151 & -83 \\
11 & 139 & -26 & -192 & -991 & 416 & 62 & -36 & -139 & -192 \\
12 & 6972 & 79 & -189 & 148 & -139 & 46 & -156 & -134 & 511 \\
13 & -86 & 7074 & -5 & 133 & 300 & 278 & -516 & 86 & -5 \\
14 & 21 & -270 & -11 & 15 & -520 & -7053 & 278 & -21 & -11 \\
13 & -129 & -902 & 3 & 425 & -7061 & -507 & 176 & 129 & 3 \\
16 & -85 & -81 & -491 & -136 & 129 & -52 & 9 & -7102 & 189 \\
17 & -126 & 49 & 172 & 7111 & -409 & -35 & 5 & 126 & 172 \\
18 & 7102 & -81 & 189 & -136 & 129 & -52 & 8 & 89 & -491 \\
\hline
\end{tabular}


TABLE 22. GECMETRY ANC LCAO-MO'S FOR S-CIS-PROPENE

\begin{tabular}{|c|c|c|c|c|}
\hline $\begin{array}{l}\text { CEOMETRY } \\
\text { ATOM NUMBER }\end{array}$ & ELEMENT & $x$ & Y & $z$ \\
\hline $\begin{array}{l}1 \\
2 \\
3 \\
4 \\
5 \\
6 \\
7 \\
8 \\
9\end{array}$ & $\begin{array}{l}C \\
C \\
C \\
H \\
H \\
H \\
H \\
H \\
H\end{array}$ & $\begin{array}{l}0.0 \\
0.0 \\
-1.316358184 \\
0.935307370 \\
-0.935307371 \\
0.935307371 \\
-1.887929125 \\
-1.111183299 \\
-1.887929125\end{array}$ & $\begin{array}{l}0.0 \\
0.0 \\
-0.000000001 \\
0.0 \\
0.0 \\
0.000000000 \\
-0.089980620 \\
-0.00000000 \\
0.889980619\end{array}$ & $\begin{array}{r}0.0 \\
1.339999199 \\
2.095998951 \\
-0.53999962 \\
-0.53999962 \\
1.879999161 \\
1.836 t 75175 \\
3.171646104 \\
1.836675176\end{array}$ \\
\hline
\end{tabular}

EXPANSICNS IN SLATER BASIS

$\begin{array}{rrrrrrrrrrr} & & 1 & 2 & 3 & 4 & 5 & t & 7 & 8 & 9 \\ 1 & 2 S & -4000 & 3261 & 4045 & 71 & 3261 & -188 & -207 & -71 & 242 \\ 1 & P X & -4745 & 39 & -4745 & 1 & 39 & -643 & 630 & -1 & 104 \\ 1 & P Y & 0 & -5039 & 0 & -583 & 5039 & 0 & 0 & -583 & 0 \\ 1 & P Z & 3285 & 3758 & -3252 & 13 & 3758 & 39 & -27 & -13 & 163 \\ 2 & 2 S & 1 \epsilon 8 & 3209 & -260 & 86 & 3209 & 4036 & 3897 & -86 & 0 \\ 2 & P X & -509 & 119 & -543 & 193 & 119 & -4771 & 4881 & -193 & 215 \\ 2 & P Y & 0 & -4915 & 0 & 6 C 8 & 4915 & 0 & 0 & 608 & 0 \\ 2 & P Z & -106 & -3839 & 0 & 150 & -3835 & 3240 & 3146 & -150 & 350 \\ 3 & 2 S & -262 & -22 & -161 & -3590 & -22 & 3650 & -121 & 3590 & 3532 \\ 3 & P X & -336 & -49 & -165 & 3276 & -49 & 5249 & 173 & -3276 & 838 \\ 3 & P Y & 0 & -477 & 0 & 4838 & 477 & 0 & 0 & 4838 & 0 \\ 3 & P 2 & 60 & -307 & 17 & 1335 & -307 & -2503 & 407 & -1335 & 5959 \\ 4 & 1 S & -7071 & 7 & -275 & -50 & 7 & 517 & -305 & 50 & -145 \\ 5 & 15 & 269 & 12 & 7054 & -13 & 12 & -277 & 523 & 13 & 26 \\ 6 & 15 & 307 & -3 & 518 & -239 & -3 & -177 & 7062 & 239 & -309 \\ 7 & 15 & -18 & -393 & -3 & -7052 & 263 & -2 & 224 & -100 & -123 \\ 8 & 15 & 136 & 258 & 98 & 129 & 258 & -14 & -297 & -129 & 1133 \\ 9 & 15 & -18 & 263 & -3 & 101 & -393 & -2 & 224 & 7092 & -123\end{array}$

EXPANSICAS IN HYORID BASIS

\begin{tabular}{|c|c|c|c|c|c|c|c|c|c|}
\hline & 1 & 2 & 3 & 4 & 5 & 6 & 7 & 8 & 9 \\
\hline $\begin{array}{l}1 \\
2\end{array}$ & $\begin{array}{r}-700 t \\
-295\end{array}$ & $\begin{array}{l}37 t \\
32 \mathrm{C}\end{array}$ & $\begin{array}{r}308 \\
7018\end{array}$ & $\begin{array}{l}36 \\
34\end{array}$ & $\begin{array}{l}376 \\
320\end{array}$ & $\begin{array}{r}-579 \\
329\end{array}$ & $\begin{array}{r}337 \\
-554\end{array}$ & $\begin{array}{l}-36 \\
-34\end{array}$ & $\begin{array}{r}146 \\
0\end{array}$ \\
\hline 3 & 263 & -61 & $-22 t$ & -376 & 7064 & -54 & -100 & -449 & 193 \\
\hline 4 & 263 & 7064 & $-2 ? h$ & 449 & -61 & -53 & -100 & 376 & 193 \\
\hline 5 & 413 & 201 & 234 & -25 & 201 & 7027 & 83 & 26 & -10 \\
\hline$t$ & $-30 t$ & 370 & -533 & 248 & 370 & 279 & 6986 & -248 & 295 \\
\hline 7 & 130 & 51 & -106 & 378 & 7003 & -223 & -225 & 482 & -202 \\
\hline e & $13 \mathrm{C}$ & 7003 & -106 & -482 & 50 & -223 & -225 & -378 & -202 \\
\hline 9 & -409 & 84 & -211 & 83 & 84 & 7039 & -106 & -83 & -185 \\
\hline 10 & & 413 & -5 & -6983 & -261 & 68 & -224 & 140 & 139 \\
\hline 11 & -133 & -280 & -91 & -139 & -280 & 204 & 313 & 139 & 6972 \\
\hline 12 & & $-2 \in 1$ & -9 & -140 & 413 & 68 & -224 & 6983 & 139 \\
\hline 13 & -7071 & 7 & -275 & -50 & 7 & 517 & -305 & 50 & -145 \\
\hline 14 & 265 & 12 & 7054 & -13 & 12 & -277 & 523 & 13 & 26 \\
\hline 15 & 307 & -3 & 518 & -239 & -3 & -177 & 7062 & 239 & -309 \\
\hline 16 & -18 & -393 & -3 & -7092 & 263 & -2 & 224 & -100 & -123 \\
\hline 17 & $\begin{array}{r}126 \\
-18\end{array}$ & $\begin{array}{l}258 \\
263\end{array}$ & $\begin{array}{l}98 \\
-3\end{array}$ & $\begin{array}{l}129 \\
101\end{array}$ & $\begin{array}{r}258 \\
-209\end{array}$ & $\begin{array}{r}-14 \\
-2\end{array}$ & -297 & -129 & $\begin{array}{r}7133 \\
-123\end{array}$ \\
\hline 18 & & & & & -293 & -2 & 224 & 7092 & -123 \\
\hline
\end{tabular}


TABLE 23. GECMETRY ANO LCAO-MO'S FOR BUTENE

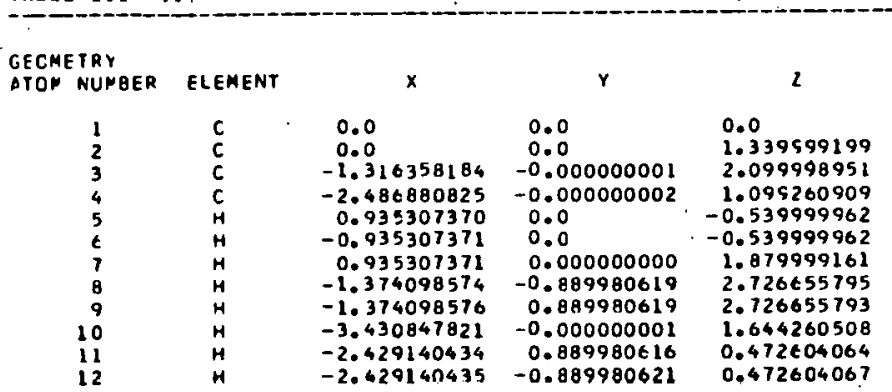

EXPANSICNS IN SLATER BASIS

\begin{tabular}{|c|c|c|c|c|c|c|c|c|c|c|c|c|c|}
\hline & & 1 & 2 & 3 & 4 & 5 & t & 7 & 8 & 9 & 10 & 11 & 12 \\
\hline & $\begin{array}{l}25 \\
P X\end{array}$ & & & -57 & $\begin{array}{r}138 \\
19\end{array}$ & $\begin{array}{r}-3270 \\
-40\end{array}$ & $\begin{array}{r}-138 \\
-79\end{array}$ & $\begin{array}{r}-3270 \\
-40\end{array}$ & & $\begin{array}{l}4 \\
3\end{array}$ & 84 & 219 & \\
\hline & p & 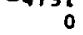 & & & 599 & -50 & -599 & 50 & 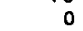 & & -3 & 0 & \\
\hline & $D 2$ & 3268 & -3282 & 5 & & & -105 & & 61 & & - & -28 & -2 \\
\hline 2 & 2 & -2 & & 2 & & & & -3 & & -8 & & & \\
\hline & 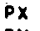 & 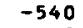 & & & & & -92 & & & & - & & \\
\hline 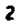 & PY & & & & 6 & $-4 y$ & o. & & & & & 0 & \\
\hline & $P Z$ & & 10 & 29 & 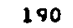 & 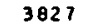 & -1 & & & -5 & & 3140 & \\
\hline & 25 & -17 & 27 & & 344 & 43 & -34 & 43 & -36 & -6 & 6 & -83 & \\
\hline & $p x$ & -137 & 306 & 26 & -36 & 7 & & 7 & 46 & 9 & - & 2 & \\
\hline & PY & & & & 47 & -479 & & 479 & & -42 & -4 & 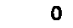 & \\
\hline & P2 & 118 & -110 & 9 & 6 & 252 & $-3 c$ & & 6 & -1 & & 327 & 27 \\
\hline & 25 & -53 & & -2 & -6 & 103 & & 103 & -3 & 36 & -36 & & \\
\hline & $P x$ & 192 & 22 & & -14 & & I & 20 & & & & & -35 \\
\hline & Pr & & 0 & 0 & 44 & 46 & & -46 & & -48 & -40 & & \\
\hline & PI & -107 & 40 & -2814 & 22 & 9 & -2 & 95 & -3949 & & 35 & & \\
\hline & 15 & -20 & 7074 & & -8 & -1 & 85 & -1 & -126 & & & & \\
\hline & 15 & 7040 & -27 & & & -10 & -2 & -10 & & 97 & -9 & & 25 \\
\hline & 15 & 499 & -29 & 13 & -14 & 15 & & 15 & -437 & & & & $1 \mathrm{e}$ \\
\hline & 15 & 34 & 3 & 15 & -10 & -473 & -1105 & 17 & & -147 & -4 & & 2 \\
\hline & 18 & 34 & -78 & & & & & -47 & & & & & \\
\hline & 15 & 179 & $-3 \theta$ & -114 & -16 & -79 & 16 & -79 & 25 & -1 & & -113 & -411 \\
\hline & 15 & $\begin{array}{l}-2 \\
-2\end{array}$ & & & $\begin{array}{r}-15 \\
43\end{array}$ & -3 & -4 & & B & & & & 36 \\
\hline & & -2 & & & 438 & & 150 & -3 & 8 & 7113 & 120 & 14 & 136 \\
\hline
\end{tabular}

EXPANSICNS IN HYBRID GASIS

\begin{tabular}{|c|c|c|c|c|c|c|c|c|c|c|c|c|}
\hline & 1 & 2 & 3 & 4 & 5 & 6 & 7 & 8 & 9 & 10 & 11 & 12 \\
\hline 1 & $33 t$ & 7004 & -47 & 93 & -384 & -93 & -384 & 117 & -83 & 83 & 8 & 561 \\
\hline 2 & 7027 & 280 & -62 & -19 & -326 & 19 & -326 & 17 & -88 & 88 & -558 & -331 \\
\hline 3 & -220 & -267 & 6 & -306 & -7002 & -541 & 57 & 100 & -6 & -43 & -106 & 62 \\
\hline 4 & -226 & -267 & 6 & 541 & 56 & 306 & -7062 & 100 & 43 & 6 & -106 & 62 \\
\hline 5 & 245 & -423 & -344 & -2 & -216 & 2 & -216 & -88 & -119 & 119 & 70 & -7014 \\
\hline$t$ & -518 & 299 & -127 & $12 \mathrm{~A}$ & $-3 A 2$ & -128 & -382 & 471 & -28 & 28 & 6978 & -314 \\
\hline 1 & -109 & -131 & 0 & 324 & -7005 & 564 & -47 & -134 & 45 & 62 & -227 & 235 \\
\hline 8 & -109 & -131 & & -564 & -47 & -324 & -7006 & -134 & -62 & -45 & -227 & 235 \\
\hline 9 & -21 & 70 & -141 & 167 & 485 & -6932 & -191 & -145 & 176 & 431 & 117 & -186 \\
\hline 10 & -240 & 417 & 340 & -129 & -82 & 129 & -82 & 14 & 122 & -122 & -214 & -7016 \\
\hline 11 & -6.3 & 0 & 7 & -85 & -125 & 85 & -125 & -7046 & 9 & -8 & -287 & 114 \\
\hline 12 & -20 & 70 & -141 & 6932 & -191 & -167 & 485 & -145 & -431 & -176 & 117 & -186 \\
\hline 13 & -217 & 52 & -6963 & 179 & 17 & -179 & 77 & -164 & 176 & -176 & 114 & 453 \\
\hline 14 & 35 & -2 & -130 & 267 & 38 & 466 & -27 & -91 & 133 & -6987 & -15 & $-15 t$ \\
\hline 15 & 39 & 26 & 126 & 0 & 119 & 0 & 119 & -7053 & -94 & 94 & 320 & -62 \\
\hline 16 & 35 & -2 & -130 & -466 & -27 & -167 & 30 & -91 & 6987 & -133 & -15 & -150 \\
\hline 11 & -283 & 7074 & 40 & -85 & -1 & 85 & -1 & -126 & 18 & -78 & -296 & -504 \\
\hline 18 & 1040 & -274 & 31 & 28 & -10 & -28 & -10 & 13 & 97 & -97 & 525 & 290 \\
\hline 19 & 495 & -295 & 134 & -141 & 15 & 141 & 15 & -437 & 33 & -33 & 1078 & 187 \\
\hline 20 & 34 & -78 & 155 & -103 & -473 & -7105 & 177 & 32 & -147 & -414 & -132 & 23 \\
\hline 21 & 34 & -78 & 155 & 1105 & 177 & 103 & -473 & 32 & 414 & 147 & -132 & 23 \\
\hline 22 & 178 & $-3 e$ & -7145 & -163 & -79 & 163 & -79 & 25 & -141 & 141 & -113 & -411 \\
\hline 23 & -23 & 7 & 138 & -150 & -3 & -438 & 6 & B & -120 & -7113 & 14 & 136 \\
\hline 3 & -25 & 7 & 138 & 438 & 6 & 150 & -3 & 8 & 7113 & 120 & 14 & 136 \\
\hline
\end{tabular}


TARIE 24. GEOMETRY AND LCAC-MO'S FOR S-CIS-BUTENE

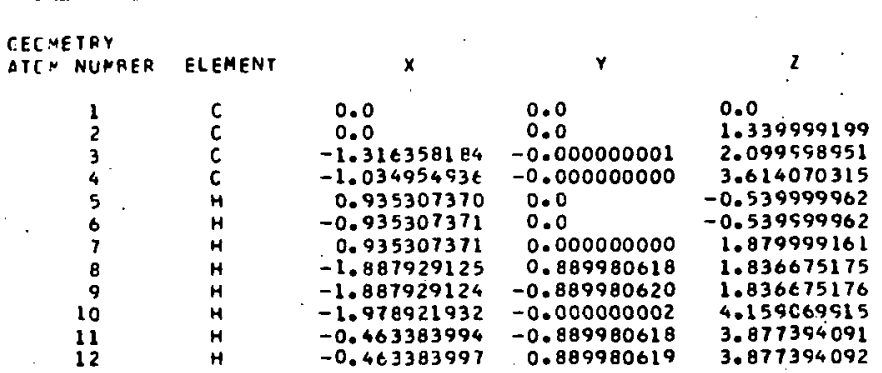

ExPAASICAS IN SLATER EASIS

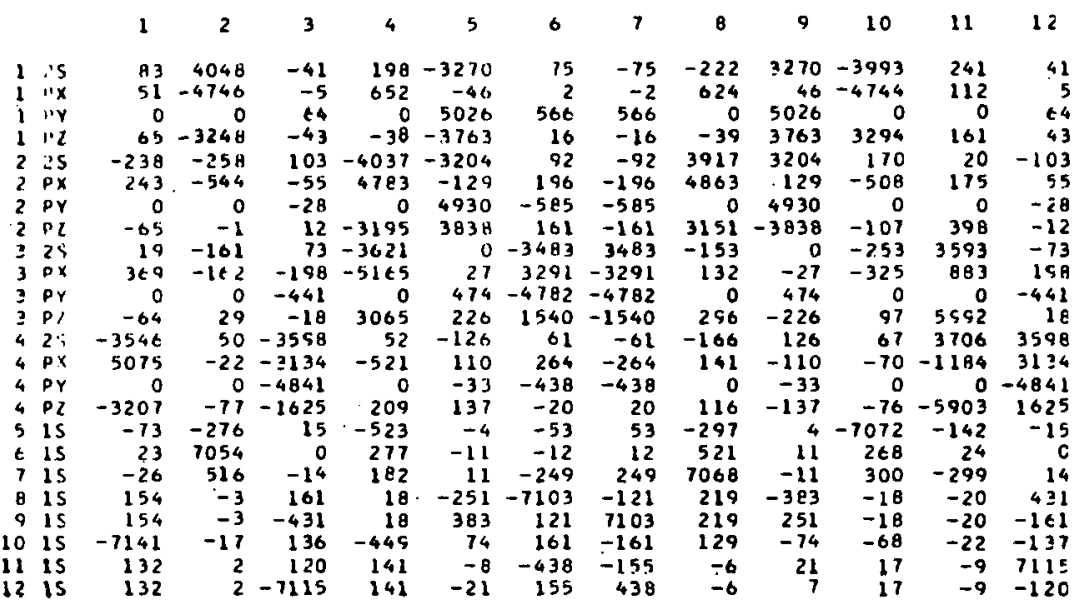

EXPANSICNS IN HYBRIO EASIS

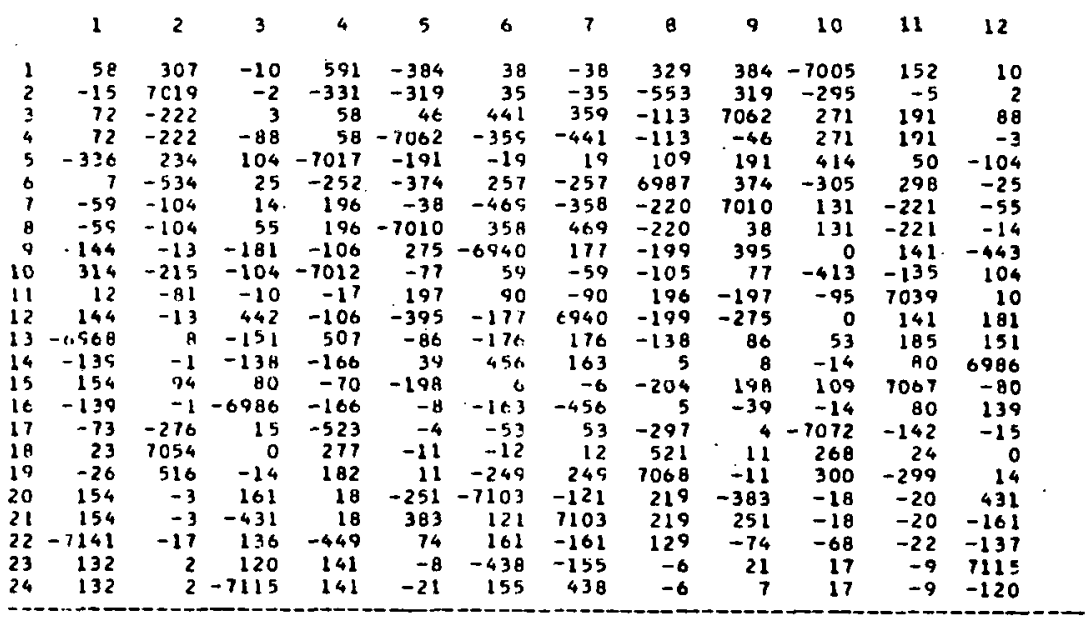


TABLE 25. GECMETRY ANO LCAO-MO'S FOR 2-EUTENE

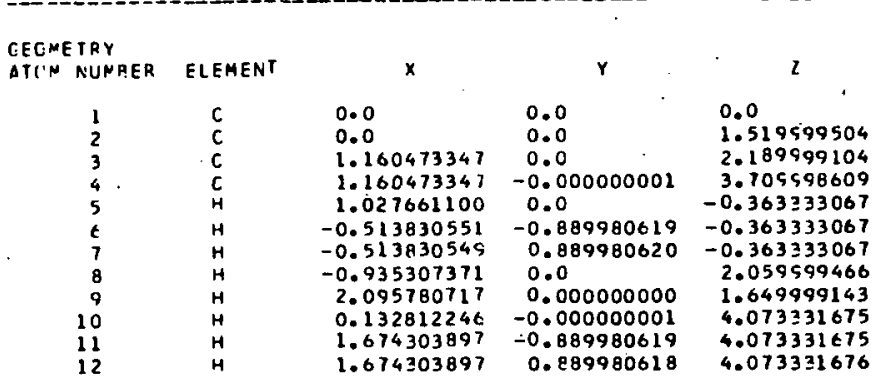

EX'ANSIENS IN SLATER BASIS

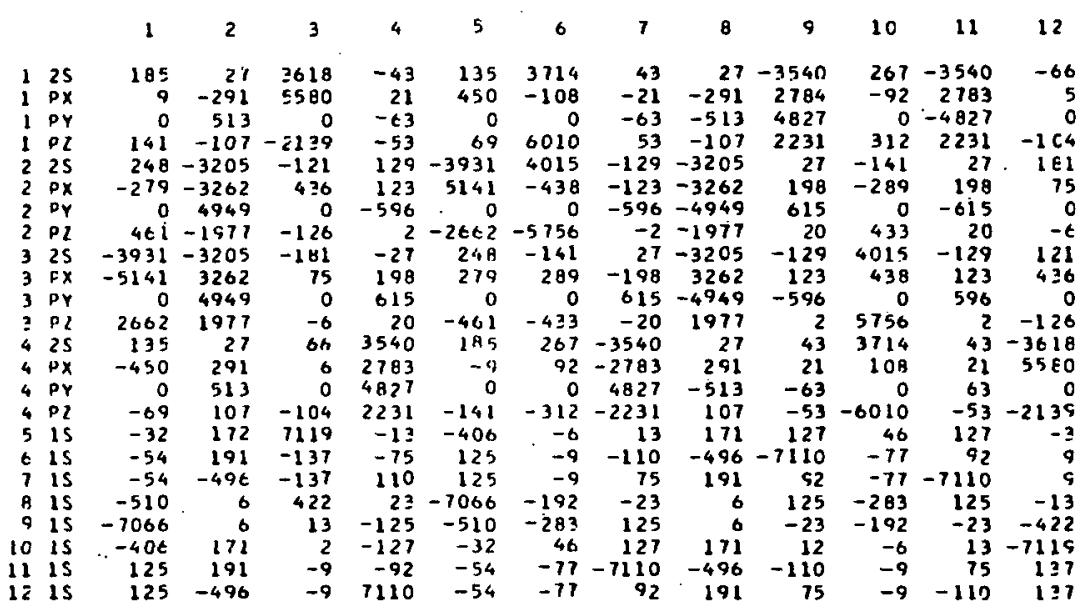

EXPANSTCNS IN MYBRIO EASIS

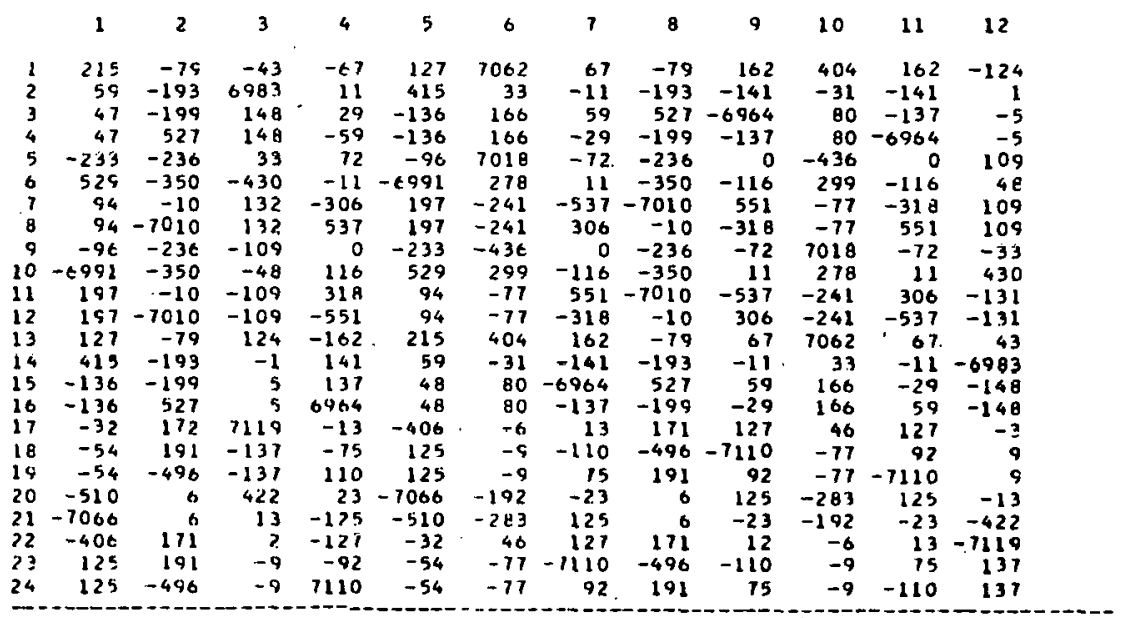


IARLE 2E. GECMETRY ANC LCAO-MO'S FCR ISCQUTENE்

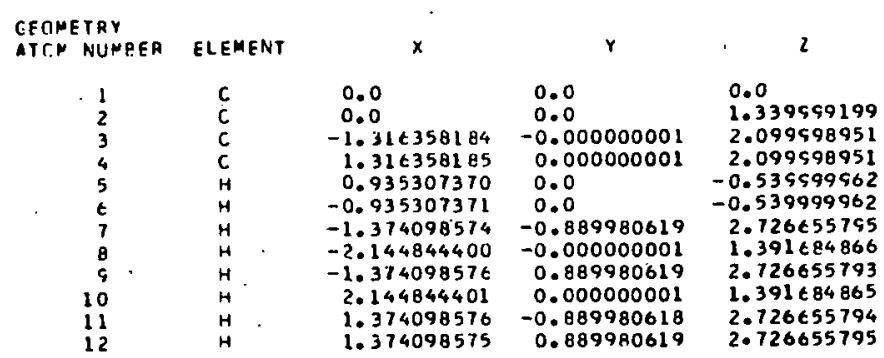

EXPANSICNS IN SLATER BASIS

\begin{tabular}{|c|c|c|c|c|c|c|c|c|c|c|c|c|c|}
\hline & & 1 & 2 & 3 & 4 & 5 & $\epsilon$ & 7 & 8 & s & 10 & 11 & 12 \\
\hline l & $\begin{array}{l}25 \\
P X\end{array}$ & $\begin{array}{r}3263 \\
0\end{array}$ & $\begin{array}{l}136 \\
-74\end{array}$ & $\begin{array}{r}-188 \\
50\end{array}$ & $\begin{array}{r}-188 \\
-50\end{array}$ & $\begin{array}{r}136 \\
-74\end{array}$ & $\begin{array}{r}-136 \\
-74\end{array}$ & $\begin{array}{l}-184 \\
-645\end{array}$ & $\begin{array}{r}136 \\
74\end{array}$ & $\begin{array}{r}3263 \\
0\end{array}$ & $\begin{array}{r}-4028 \\
4734\end{array}$ & $\begin{array}{r}-184 \\
645\end{array}$ & $\begin{array}{l}-4028 \\
-4724\end{array}$ \\
\hline$i$ & PY & $50 \in E$ & 573 & 0 & 0 & -573 & -573 & 0 & -573 & -5066 & 0 & 0 & 0 \\
\hline$i$ & $P Z$ & 372 & 96 & -57 & -57 & 96 & -96 & 53 & 96 & 3725 & 3264 & 53 & $32 \div 4$ \\
\hline 2 & 25 & 3 & -40 & -84 & -84 & -40 & 40 & 3946 & -40 & 3141 & 209 & 3546 & $2 \mathrm{Cs}$ \\
\hline 2 & $p x$ & & -49 & 240 & -240 & -49 & -49 & -4882 & 49 & 0 & 431 & 4882 & $-4 \geq 1$ \\
\hline 2 & PY & 4838 & -612 & 0 & & 612 & 612 & 0 & 612 & $-48 \geq 8$ & 0 & 0 & 0 \\
\hline 7 & $p i$ & -3905 & 157 & -259 & -259 & 157 & -157 & 3137 & 157 & -3905 & -77 & 3137 & -17 \\
\hline 3 & 25 & $-2 \theta$ & -75 & 221 & $3 \in 05$ & -75 & -3558 & 3702 & 3558 & -28 & 192 & -119 & $-2 \in E$ \\
\hline$?$ & $p x$ & -57 & -50 & 192 & $-4 A 75$ & -50 & 520 & 5209 & -520 & -57 & 116 & 227 & -312 \\
\hline 3 & PY & $4 \mathrm{~A}_{4}$ & 34 & 0 & 0 & -34 & 4840 & & 4840 & -484 & 0 & 0 & 0 \\
\hline 3 & $P$ i & -307 & 38 & -205 & -3753 & 38 & -3519 & -2944 & 3519 & -307 & -89 & 406 & $\varepsilon 2$ \\
\hline 4 & 25 & -28 & 3558 & $3 \in C 5$ & 221 & 3558 & 75 & -119 & -75 & -27 & $-2<8$ & 3702 & 152 \\
\hline 4 & $P x$ & 57 & 520 & 4075 & -152 & 320 & -50 & -2 & 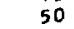 & 57 & 312 & -5209 & -116 \\
\hline 4 & py & 404 & -4040 & 0 & 0 & 4040 & -34 & 0 & -34 & -404 & 0 & 0 & 0 \\
\hline 4 & $p_{2}$ & -307 & 3519 & -3753 & $-2 c 5$ & 3519 & -38 & 406 & 38 & -307 & 82 & $-2 c$ & -89 \\
\hline 5 & is & 2 & 15 & 33 & 124 & 15 & 82 & 517 & -82 & 2 & 292 & -277 & -7019 \\
\hline 6 & is & 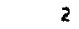 & -82 & 124 & 33 & -82 & -15 & -27 & 15 & ; & .7079 & 517 & 292 \\
\hline 7 & is & 487 & -12 & 4 & -138 & $3 \mathrm{e}$ & -7101 & -15 & -89 & -180 & -48 & -115 & 80 \\
\hline 8 & is & -176 & 31 & -98 & 7119 & 31 & 132 & -1 & -132 & -176 & -54 & & -41 \\
\hline 9 & 15 & -180 & 38 & & -138 & -12 & 89 & -15 & 7101 & 487 & -48 & -115 & EO \\
\hline 10 & 15 & -176 & -132 & 1119 & -98 & -132 & $-3 i$ & 407 & 31 & -176 & -41 & -23 & -54 \\
\hline 11 & 15 & 487 & 7101 & -138 & 4 & -89 & 12 & -115 & 38 & -180 & 80 & -15 & -48 \\
\hline & is & -180 & -89 & -138 & 4 & 7101 & -38 & 115 & -12 & 487 & 80 & -15 & $-4 \varepsilon$ \\
\hline
\end{tabular}

EXPANSIENS IN HYGRID BASIS

\begin{tabular}{|c|c|c|c|c|c|c|c|c|c|c|c|c|}
\hline & 1 & 2 & 3 & 4 & 5 & 6 & 7 & 8 & 9 & 10 & 11 & 12 \\
\hline$\frac{1}{2}$ & $-3 \in 2$ & -13 & -49 & -121 & -13 & -92 & $\begin{array}{r}-584 \\
328\end{array}$ & 92 & 363 & -311 & 328 & -1006 \\
\hline 2 & $\begin{array}{r}362 \\
7065\end{array}$ & $\begin{array}{r}92 \\
51 t\end{array}$ & $\begin{array}{l}-121 \\
-110\end{array}$ & $\begin{array}{r}-49 \\
-110\end{array}$ & $\begin{array}{r}92 \\
-294\end{array}$ & $\begin{array}{r}13 \\
-516\end{array}$ & $\begin{array}{r}328 \\
-44\end{array}$ & $\begin{array}{r}-13 \\
-294\end{array}$ & $\begin{array}{r}362 \\
-99\end{array}$ & $\begin{array}{r}-7006 \\
239\end{array}$ & $\begin{array}{r}-384 \\
-44\end{array}$ & $\begin{array}{r}-311 \\
239\end{array}$ \\
\hline 4 & -99 & -294 & -110 & -110 & 516 & 294 & -44 & 516 & 7065 & 239 & -44 & 239 \\
\hline 5 & 219 & 75 & -324 & 15 & 75 & -6 & 7012 & 6 & 219 & -215 & 106 & 394 \\
\hline$t$ & 215 & $t$ & 15 & -324 & 6 & -75 & 106 & 75 & 219 & 394 & 7012 & -215 \\
\hline 7 & 6558 & -540 & 115 & 115 & 325 & 540 & -200 & 325 & 115 & 129 & -200 & 129 \\
\hline 8 & 115 & 325 & iis & 115 & -540 & -325 & $-200^{\circ}$ & -540 & 6958 & 129 & -200 & 125 \\
\hline 9 & -507 & -41 & 0 & 148 & 8 & -6978 & 146 & 132 & 178 & 46 & 132 & $-7 \mathrm{e}$ \\
\hline 10 & 10 & $-4 z$ & 343 & -78 & -92 & 134 & 1033 & -134 & 76 & 222 & -65 & -404 \\
\hline 11 & 196 & -26 & 95 & 6992 & -26 & -141 & 79 & 141 & 196 & 69 & -438 & 24 \\
\hline 12 & i ie & & & 148 & -41 & -132 & 146 & 6978 & -507 & 46 & 132 & -78 \\
\hline 13 & $19 t$ & 141 & 6992 & 99 & 141 & 26 & -438 & -26 & 196 & 24 & 79 & 69 \\
\hline 14 & 76 & -134 & -18 & 343 & -134 & 92 & -65 & -92 & 76 & -404 & 1033 & 227 \\
\hline $1 \leqslant$ & -507 & OSTA & 148 & 0 & 132 & 41 & 131 & 8 & 178 & -78 & 146 & $.4 \mathrm{t}$ \\
\hline 16 & 178 & 132 & 148 & 0 & 6978 & -8 & 131 & -41 & -507 & -78 & 146 & 46 \\
\hline it & 2 & 15 & 34 & 126 & 15 & B2 & 517 & -82 & 2 & 292 & -217 & -7079 \\
\hline is & 2 & -A2 & $1<4$ & 33 & -82 & -13 & -277 & 15 & 2 & -1079 & 317 & 292 \\
\hline 19 & 487 & -12 & 4 & $-13 A$ & 30 & -1101 & -15 & -89 & -180 & -48 & -115 & 80 \\
\hline 20 & $-17 t$ & 31 & -98 & 1119 & 31 & 132 & -13 & -132 & -176 & -54 & 407 & -41 \\
\hline 21 & -180 & 38 & 4 & -136 & -12 & A9 & -15 & 7101 & 487 & -48 & -115 & 80 \\
\hline 22 & $-17 t$ & -132 & 1119 & -98 & -132 & $-3 i$ & 407 & 31 & -176 & -41 & -13 & -54 \\
\hline 23 & 487 & 7101 & $-13 A$ & 4 & -89 & 12 & -115 & 38 & -180 & no & -15 & -48 \\
\hline 24 & $-18 c$ & -89 & $-13 A$ & 4 & 7101 & $-3 \ddot{p}$ & -115 & -12 & 487 & 80 & -15 & -48 \\
\hline
\end{tabular}


TABLE 21. GECMETRY ANO LCAO-MO'S FOR GUTACIENE

\begin{tabular}{|c|c|c|c|c|}
\hline $\begin{array}{l}\text { GECMETRY } \\
\text { ATOW NUMBER }\end{array}$ & ELEMENT & $x$ & $\checkmark$ & 2 \\
\hline $\begin{array}{r}1 \\
2 \\
3 \\
4 \\
5 \\
6 \\
7 \\
8 \\
9 \\
10\end{array}$ & $\begin{array}{l}C \\
C \\
C \\
C \\
H \\
H \\
H \\
H \\
H \\
H\end{array}$ & $\begin{array}{r}0.0 \\
0.0 \\
-1.264396296 \\
-1.26439629 t \\
0.935307370 \\
-0.935307371 \\
0.935307371 \\
-2.199703667 \\
-2.199703606 \\
-0.329088925\end{array}$ & $\begin{array}{l}0.0 \\
0.0 \\
-0.000000001 \\
-0.000000000 \\
0 . c \\
0.0 \\
0.000000000 \\
-0.000000001 \\
-0.000000001 \\
0.000000001\end{array}$ & $\begin{array}{l}0.0 \\
1.339999199 \\
2.069958741 \\
3.409997941 \\
-0.539999962 \\
-0.539599962 \\
1.879999161 \\
1.525998780 \\
3.949997904 \\
3.945597903\end{array}$ \\
\hline
\end{tabular}

EXPANSICNS IN SLATER GASIS

\begin{tabular}{|c|c|c|c|c|c|c|c|c|c|c|c|c|}
\hline & & $\boldsymbol{I}$ & 2 & 3 & 4 & 5 & 6 & 7 & e & 5 & 10 & 11 \\
\hline & & & 15 & & 277 & 54 & 4061 & 39 & & -186 & & \\
\hline & D) & & 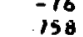 & 0 & 9 & 7 & & & -49 & & $\begin{array}{l}6 \\
0\end{array}$ & \\
\hline 1 & PI & -11 & -11 & 52 & & & 3242 & 3306 & & 68 & -3 & -6 \\
\hline & 2 & & 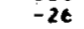 & & & & & & & 4 & & 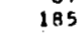 \\
\hline & $P x$ & -21 & -4 & & & 4 & & & & & 32 & 11 \\
\hline & Py & 0 & -11 & 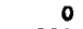 & 49 & -7 & 0 & 0 & -49 & & 0 & \\
\hline & P2 & 8 & -20 & 339 & -38 & 2 & 9 & -123 & -38 & 3 & 3 & \\
\hline & 25 & -156 & -31 & 209 & & & 185 & -2 & & & -1 & 25 \\
\hline & $p x$ & -482 & i & & $-4 c$ & 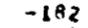 & 3 & -275 & -8 & 478 & 259 & -530 \\
\hline & Py & & -490 & & 711 & -49 & & 0 & -7 & & 0 & \\
\hline & 02 & -123 & -380 & 2133 & -2 & 38 & -42 & 87 & -2 & -314 & 39 & - \\
\hline & 25 & 3982 & -32 & 2 & 15 & 32 & -6 & 88 & 154 & -18 & -163 & 061 \\
\hline & $\begin{array}{l}P x \\
P y\end{array}$ & & -498 & 62 & $\begin{array}{r}-76 \\
-758\end{array}$ & -6 & 1 & -63 & -1 & 65 & 34 & 74 \\
\hline & PI & $230 t$ & 3760 & -32 & -111 & $\begin{array}{l}-64 A \\
-376\end{array}$ & 67 & -71 & -111 & -6 & 52 & 69 \\
\hline & is & 8 & 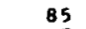 & -109 & & -85 & $2 \mathrm{Al}$ & -7071 & & & -298 & 64 \\
\hline & is & -31 & -17 & -28 & 10 & 17 & $-7 C 50$ & & 1 & -2 & 522 & -3 \\
\hline & is & 3 & 160 & -429 & -1 & -160 & & & & & 7064 & -16 \\
\hline & 15 & 30 & & 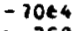 & $-1 \in 0$ & 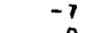 & -16 & - & 16 & & 429 & -509 \\
\hline & 15 & & & & & & & & & & 09 & 81 \\
\hline & 15 & 271 & -10 & 522 & 17 & 10 & -3 & 31 & 17 & -211 & 19 & 705 \\
\hline
\end{tabular}

EXPACSICAS IN MYBRIO BASIS

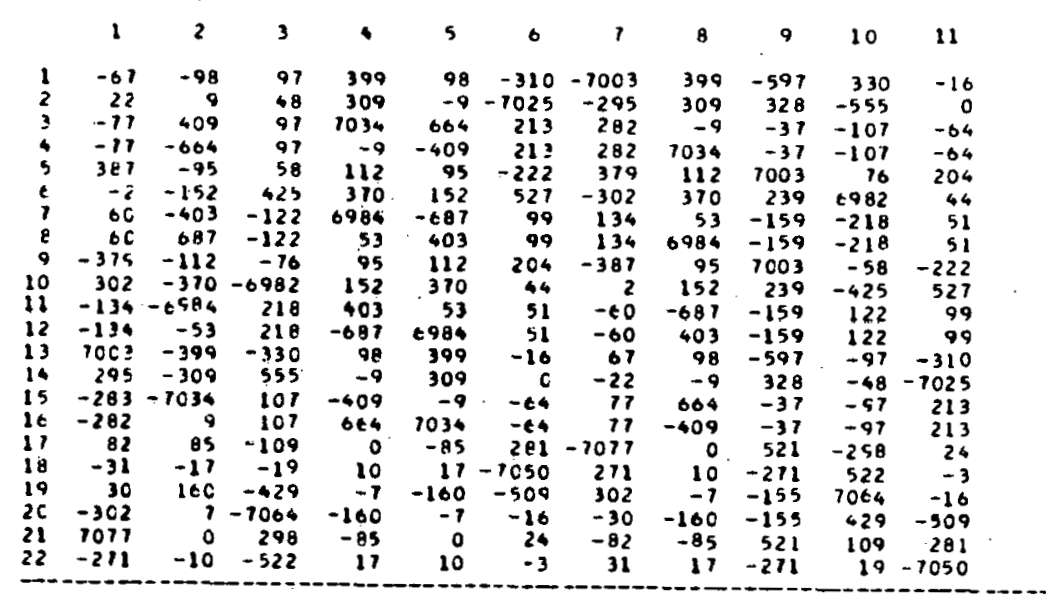


TABLE 28. GECMETRY ANC LCAO-MD'S FCR PRCPYNE

\begin{tabular}{|c|c|c|c|c|}
\hline $\begin{array}{l}\text { CECNETRY } \\
\text { ATCN NUMBER }\end{array}$ & ELEMENT & $x$ & $Y$ & 2 \\
\hline $\begin{array}{l}1 \\
2 \\
3 \\
4 \\
5 \\
6 \\
7\end{array}$ & $\begin{array}{l}H \\
C \\
C \\
C \\
H \\
H \\
H\end{array}$ & $\begin{array}{l}0.0 \\
0.0 \\
-0.000000000 \\
-0.000000001 \\
1.027661100 \\
-0.513830551 \\
-0.513830551\end{array}$ & $\begin{array}{l}0.0 \\
0.0 \\
0.0 \\
0.0 \\
0 . \mathrm{C} \\
0.889980619 \\
-0.889980619\end{array}$ & $\begin{array}{l}0.0 \\
1.059999466 \\
2.259999275 \\
3.719958360 \\
4.083331426 \\
4.083331426 \\
4.083331426\end{array}$ \\
\hline
\end{tabular}

EXPANSICNS IN SLATER BASIS

$\begin{array}{rrrrrrrrrr} & & 1 & 2 & 2 & 4 & 5 & 6 & 7 & 8 \\ 1 & 15 & -6873 & 36 & -31 & 31 & -3 t & -36 & 350 & -31 \\ 2 & 25 & -4867 & -97 & 3248 & -3248 & 97 & 97 & -596 & 3248 \\ 2 & P X & 0 & -352 & -2444 & 2320 & 352 & -704 & 0 & 5765 \\ 2 & P Y & 0 & -010 & -4668 & -5316 & -610 & 0 & 0 & -648 \\ 2 & P Z & 5345 & -41 & 2460 & -2460 & 41 & 41 & -75 & 2460 \\ 3 & 25 & 606 & 58 & 2192 & -3192 & -58 & -58 & 4792 & 3192 \\ 3 & P X & 0 & 380 & -3379 & 2276 & -380 & 761 & 0 & 5656 \\ 3 & P Y & 0 & 059 & -4580 & -5216 & 659 & 0 & 0 & -636 \\ 3 & P Z & -199 & -109 & -2553 & 2553 & 109 & 109 & 5265 & -2553 \\ 4 & 2 S & -171 & -3537 & -29 & 29 & 3537 & 3537 & 3668 & -29 \\ 4 & D X & 0 & 2794 & -324 & 218 & -2794 & 5588 & 0 & 542 \\ 4 & P Y & 0 & 4839 & -439 & -500 & 4835 & 0 & 0 & -61 \\ 4 & P 2 & 221 & -2240 & -64 & 65 & 2240 & 2240 & -5946 & -64 \\ 5 & 15 & .24 & 100 & 315 & -226 & -100 & 7078 & 18 & -413 \\ 6 & 15 & 24 & 100 & 226 & 413 & 7078 & -100 & 18 & 315 \\ 7 & 15 & 24 & -7078 & -413 & -315 & -100 & -100 & 18 & 226\end{array}$

EXPANSICNS IN HYBRID EASIS

$\begin{array}{rrrrrrrrr}. & 1 & 2 & 3 & 4 & 5 & 6 & 7 & 8 \\ 1 & -6873 & 36 & -31 & 31 & -36 & -36 & 350 & -31 \\ 2 & -7229 & -35 & 369 & -369 & 35 & 35 & -345 & 369 \\ 3 & 1 & -287 & -25 & 25 & 399 & -513 & -283 & 7075 \\ 4 & 1 & 513 & 7079 & 25 & 287 & 399 & -283 & -25 \\ 5 & 1 & -399 & -25 & -7079 & -513 & 287 & -284 & -25 \\ t & 261 & -41 & 262 & -263 & 41 & 41 & 7119 & 262 \\ 7 & 33 t & 31 t & 31 & -32 & -436 & 550 & -2 & 7002 \\ 8 & 33 t & -55 C & 7002 & -31 & -216 & -436 & -2 & 32 \\ 9 & 336 & 436 & 32 & -7002 & 550 & -316 & -2 & 31 \\ 10 & -277 & 171 & 41 & -41 & -171 & -171 & 6984 & 41 \\ 11 & -21 & -134 & -298 & 212 & 134 & 6978 & 117 & 409 \\ 12 & -21 & -134 & -212 & -405 & -978 & 134 & 117 & -298 \\ 13 & -21 & -6578 & 409 & 298 & 134 & 134 & 117 & -212 \\ 14 & 24 & 100 & 315 & -226 & -100 & 7078 & 18 & -413 \\ 15 & 24 & 100 & 226 & 413 & 7078 & -100 & 18 & 315 \\ 16 & 24 & -7078 & -413 & -315 & -100 & -100 & 18 & 226 \\ -\end{array}$


TARLE 29. GECMETRY AND LCAO-MO'S FCR BUTYNE

CECMETRY

ATOH NUMBER ELEMENT

$\begin{array}{rrrll}1 & H & 0.0 & 0.0 & 0.0 \\ 2 & C & 0.0 & 0.0 & 1.059999466 \\ 3 & \mathrm{C} & -0.000000000 & 0.0 & 2.259999275 \\ 4 & \mathrm{C} & -0.000000001 & 0.0 & 3.719998360 \\ 5 & C & 1.451925888 & 0.0 & 4.233331681 \\ 6 & H & -0.513830551 & 0.089980619 & 4.083331426 \\ 7 & H & -0.513830551 & -0.889980619 & 4.083331426 \\ 8 & H & 1.965756438 & -0.889980619 & 3.869998614 \\ 9 & H & 1.945756438 & 0.889980620 & 3.869998616 \\ 10 & H & 1.451925888 & -0.000000000 & 5.323330880\end{array}$

$\begin{array}{rrrll}1 & H & 0.0 & 0.0 & 0.0 \\ 2 & H & 0.0 & 0.0 & 1.059999466 \\ 3 & \mathrm{C} & -0.000000000 & 0.0 & 2.259999275 \\ 4 & \mathrm{C} & -0.000000001 & 0.0 & 3.719998360 \\ 5 & \mathrm{C} & 1.451925888 & 0.0 & 4.233331681 \\ 6 & H & -0.513830551 & 0.089980619 & 4.083331426 \\ 7 & H & -0.513830551 & -0.889980619 & 4.083331426 \\ 8 & H & 1.965756438 & -0.889980619 & 3.869998614 \\ 9 & H & 1.945756438 & 0.889980620 & 3.869998616 \\ 10 & H & 1.45192588 \mathrm{~B} & -0.000000000 & 5.323330880\end{array}$

2

EXPANSICNS IN SLATER BASIS

$\begin{array}{rrrrrrrrrrrrr} & & 1 & 2 & 3 & 4 & 5 & 6 & 7 & 8 & 9 & 10 & 11 \\ 1 & 15 & -352 & -33 & 37 & -46 & -13 & 37 & 13 & -33 & 35 & -24 & -6874 \\ 2 & 25 & 604 & 3229 & -99 & 79 & 26 & -99 & -26 & 3229 & -99 & 3272 & -4868 \\ 2 & P X & -5 & 2956 & -365 & -187 & -77 & -365 & 77 & 2898 & 700 & -5847 & -22 \\ 2 & P Y & 0 & -4986 & -555 & 0 & -60 & 595 & -60 & 5019 & 0 & -33 & 0 \\ 2 & P Z & 77 & 2457 & -41 & 32 & 12 & -41 & -12 & 2457 & -40 & 2457 & 5342 \\ 3 & 25 & -4791 & 3212 & 66 & -260 & -78 & 66 & 78 & 3212 & 24 & 3170 & 610 \\ 3 & P X & 97 & 2866 & 398 & 180 & 69 & 398 & -69 & 2810 & -803 & -5447 & -9 \\ 3 & P Y & 0 & -4935 & 634 & 0 & 23 & -634 & 23 & 4968 & 0 & -32 & 0 \\ 3 & P Z & -5250 & -2539 & -100 & -186 & -49 & -100 & 49 & -2539 & -174 & -2586 & -199 \\ 4 & 25 & -3604 & -41 & -3431 & 7 & -79 & -3431 & 79 & -41 & -3591 & 7 & -169 \\ 4 & P X & 187 & 212 & 2964 & 117 & 110 & 2964 & -110 & 208 & -5635 & -383 & 9 \\ 4 & P Y & 0 & -470 & 4781 & 0 & 439 & -4781 & 439 & 473 & 0 & -3 & 0 \\ 4 & P Z & 5951 & -83 & -2160 & -350 & -134 & -2160 & 154 & -82 & -2207 & -11 & 224 \\ 5 & 25 & 35 & -113 & 43 & -3567 & 3598 & 43 & -3598 & -111 & -3668 & 257 & 11 \\ 5 & P X & -53 & 108 & 140 & -191 & 2988 & 140 & -2988 & 105 & 5660 & -323 & -23 \\ 5 & P Y & 0 & 33 & 435 & 0 & 4852 & -435 & 4852 & -34 & 0 & 0 & 0 \\ 5 & P Z & 536 & -17 & -242 & -5995 & -1899 & -242 & 1899 & -16 & 1942 & 37 & 22 \\ 6 & 15 & -2 & 488 & 121 & 169 & -154 & -7091 & -434 & -181 & 0 & -182 & 24 \\ 7 & 15 & -2 & -177 & -7091 & 169 & 434 & 121 & 154 & 488 & 0 & -186 & 24 \\ 8 & 15 & 135 & -4 & 149 & 132 & -118 & -442 & -1103 & 10 & 11 & 18 & 13 \\ 9 & 15 & 135 & 9 & -442 & 132 & 7103 & 249 & 118 & -3 & 11 & 18 & 12 \\ 10 & 15 & -432 & 154 & 176 & -7132 & -132 & 176 & 132 & 73 & 20 & -154 & -35\end{array}$

EXPANSIONS IN MYBRIO BASIS

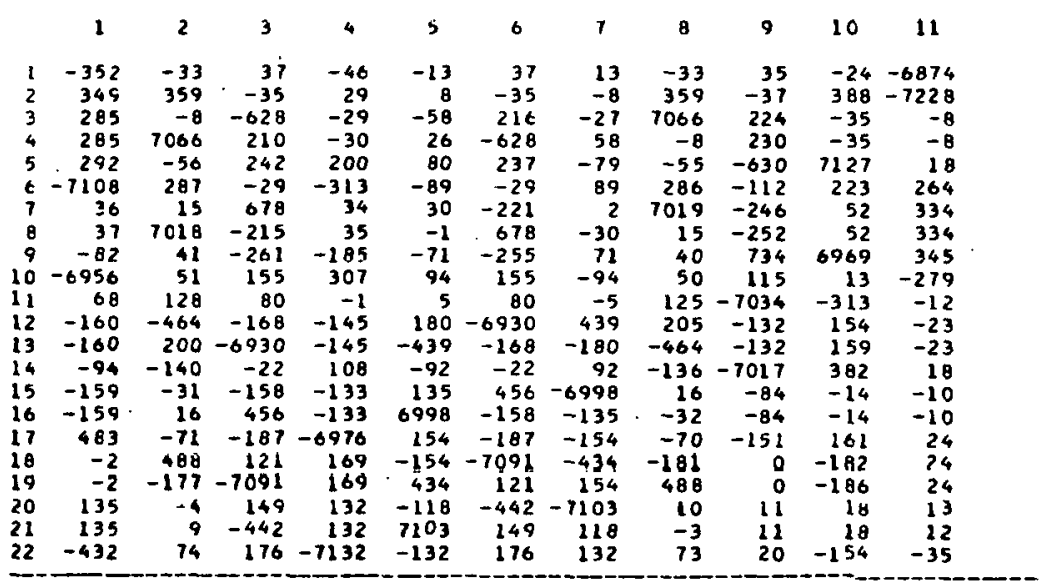


TABIF 30. CECMETRY ANC LCAO-MO'S FOR PEATYNE

GECNETRY

ATON AUMBER ELEMENT

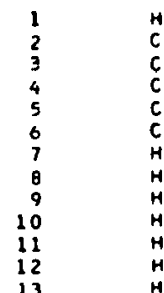

$H$
$C$
$C$
$C$
$C$
$C$
$H$
$H$
$H$
$H$
$H$
$H$
$H$

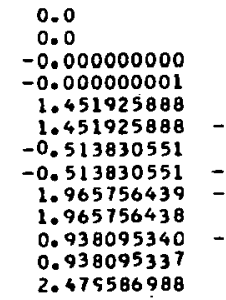

0.0
0.0
0.0
0.0
0.0
-0.000000001
0.889980619
-0.889980619
-0.889980619
0.889980619
-0.889980621
0.889980618
0.000000002

$\boldsymbol{l}$

1.059999466

3.719998360

4.233331681

5.773331643

4.083331426

4.083331426

3.869998614

3.869998614

6.136664709

6.136664709

EXPANSICNS IN SLATER GASIS

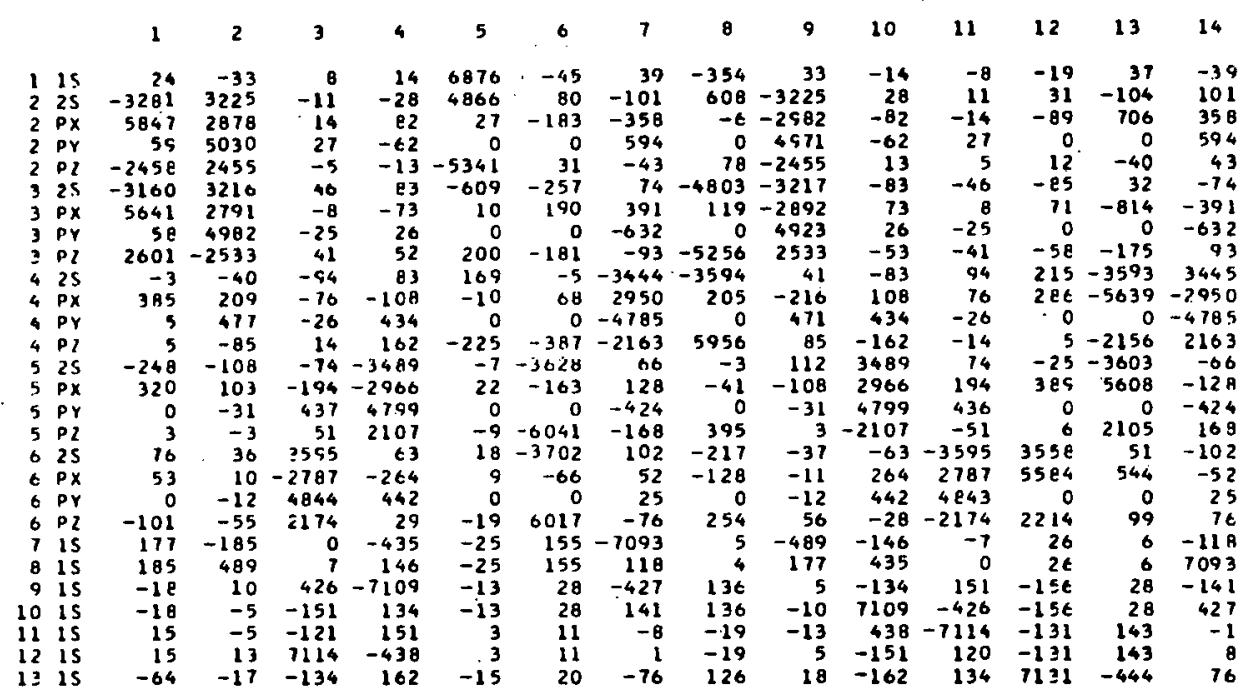

EXPANSIONS IN FYBRIO BASIS

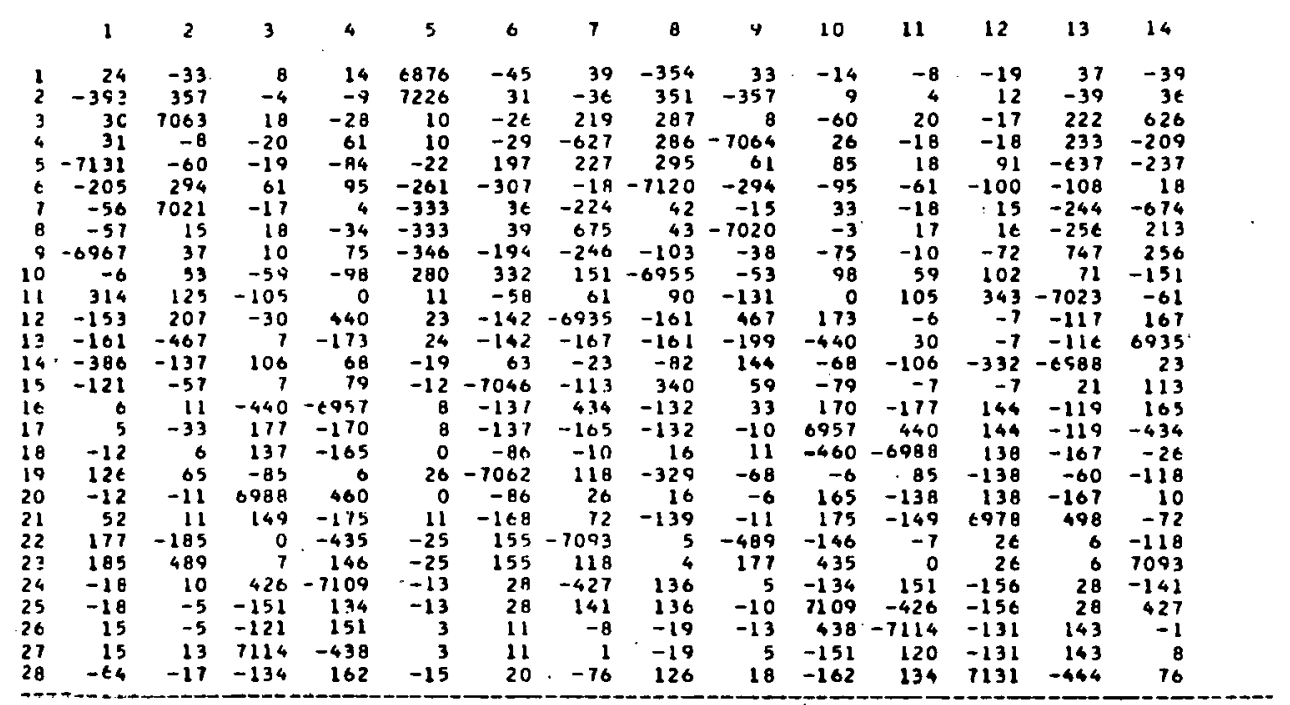


TABLE 31. GeCMETRY ANC LCAO-MO'S For VINYL aCeTrLENE

\begin{tabular}{|c|c|c|c|c|}
\hline NUMBER & ELEMENT & $x$ & $\begin{array}{r}Y \\
Y\end{array}$ & 2 \\
\hline $\begin{array}{l}1 \\
2 \\
3 \\
4 \\
5\end{array}$ & $\begin{array}{l}H \\
C \\
C \\
C \\
C\end{array}$ & $\begin{array}{l}0.0 \\
0.0 \\
-0.000000000 \\
-0.000000001 \\
1.160473347\end{array}$ & $\begin{array}{l}0.0 \\
0.0 \\
0.0 \\
0.0 \\
0.0\end{array}$ & $\begin{array}{l}0.0 \\
1.059599466 \\
2.259599275 \\
3.709999084 \\
4.379598684\end{array}$ \\
\hline $\begin{array}{l}6 \\
7 \\
8\end{array}$ & $\begin{array}{l}H \\
H \\
H\end{array}$ & $\begin{array}{r}-0.935307371 \\
2.045780717 \\
1.160473347\end{array}$ & $\begin{array}{l}0.0 \\
0.000000000 \\
-0.000000000\end{array}$ & $\begin{array}{l}4.249599047 \\
3.839998723 \\
5.459598609\end{array}$ \\
\hline
\end{tabular}

EXPANSICNS IN SLATER BASIS

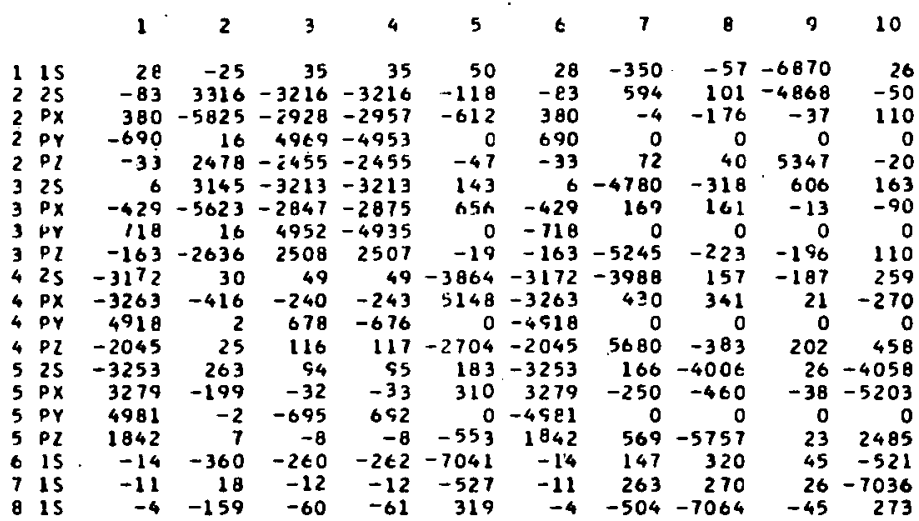

EXPANSICNS IN HYBRIO BASIS

\begin{tabular}{|c|c|c|c|c|c|c|c|c|c|c|}
\hline & 1 & 2 & 3 & 4 & 5 & 6 & 7 & 8 & 9 & 10 \\
\hline 1 & 28 & -25 & 35 & 35 & 50 & 2.8 & -350 & -57 & -6870 & 20 \\
\hline 2. & $-3 i$ & 401 & -352 & -352 & -44 & -31 & 346 & 38 & -7231 & -18 \\
\hline 3 & -379 & 0 & -20 & -7037 & -320 & 594 & 280 & -13 & -13 & 16 \\
\hline 4 & 594 & i & -7037 & -20 & -317 & -383 & 280 & -12 & -12 & is \\
\hline 5 & $-3 t 1$ & 7135 & 75 & 75 & 431 & -358 & 285 & 203 & 32 & -119 \\
\hline 6 & -116 & 170 & -310 & -311 & 82 & -116 & -7097 & -379 & 263 & 192 \\
\hline 7 & 396 & 71 & -10 & -7010 & 330 & -617 & by & 16 & 329 & -10 \\
\hline 8 & -616 & 71 & -7010 & -19 & 335 & 400 & 68 & 16 & 329 & -9 \\
\hline 9 & $41 \mathrm{E}$ & 6959 & -9 & -10 & -406 & 414 & -137 & -180 & 34.6 & 100 \\
\hline 10 & -161 & -2 & -66 & -66 & -22 & -161 & -6940 & 403 & -273 & -224 \\
\hline 11 & -359 & 322 & 246 & 248 & -6975 & $-35 \theta$ & -288 & -307 & -41 & 528 \\
\hline 12 & -39 & -186 & 413. & -545 & 216 & -6995 & 226 & 124 & -7 & 103 \\
\hline 12 & -6555 & -189 & -546 & 410 & 216 & -39 & 226 & 124 & -7 & 103 \\
\hline 14 & -312 & & 34 & 35 & 551 & -312 & -313 & -288 & $-2 i$ & -7037 \\
\hline 15 & -374 & 158 & 47 & 48 & -346 & -314 & 561 & -7014 & 34 & -313 \\
\hline $1 t$ & $-702 z$ & $20 t$ & 548 & -432 & 71 & 22 & 28 & 250 & 23 & 227 \\
\hline 17 & 22 & 203 & -434 & 547 & 14 & -7022 & 28 & 256 & 23 & 227 \\
\hline 18 & -14 & -360 & -260 & -262 & -1041 & -14 & 147 & 320 & 45 & -521 \\
\hline 19 & -11 & & -22 & -12 & -521 & -11 & 263 & 270 & 26 & -7036 \\
\hline 20 & -4 & -159 & -80 & -61 & 319 & -4 & -504 & -7064 & -45 & 273 \\
\hline
\end{tabular}


TABLE 32. GEOMETRY ANO LCAOMOIS FOR ISCPRENE

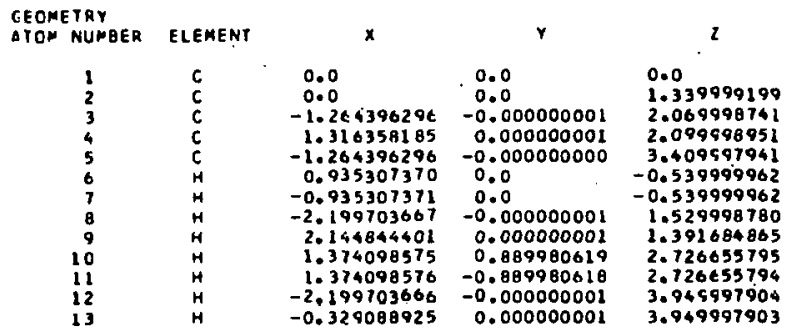

EXPANSICNS IN SLATER GASTS

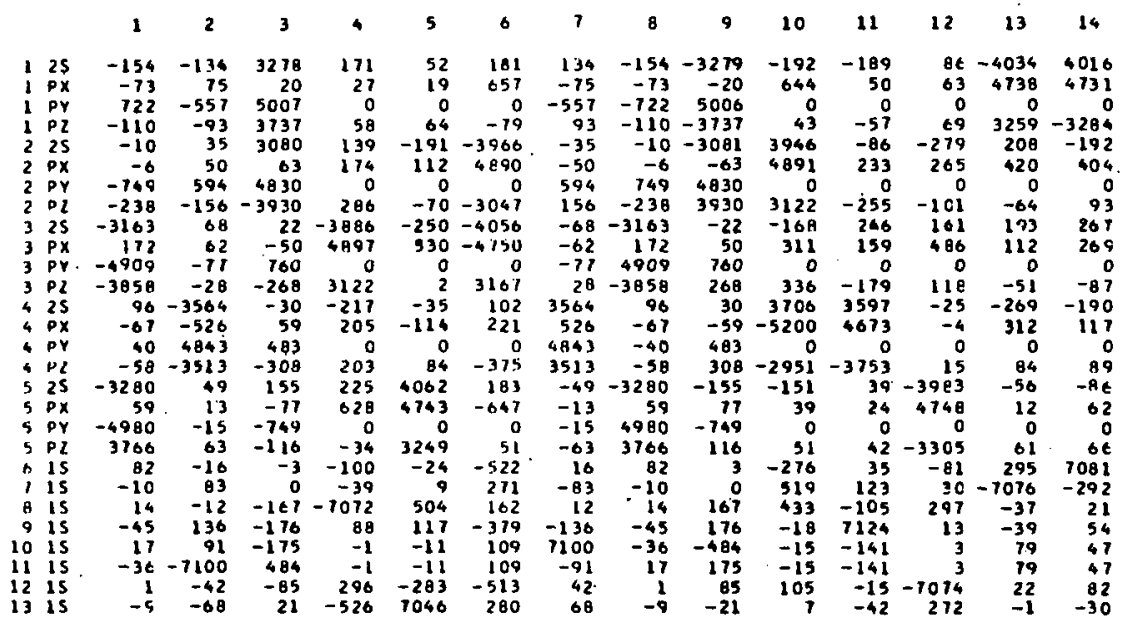

EXPANSICNS IN HYBRIO BASIS

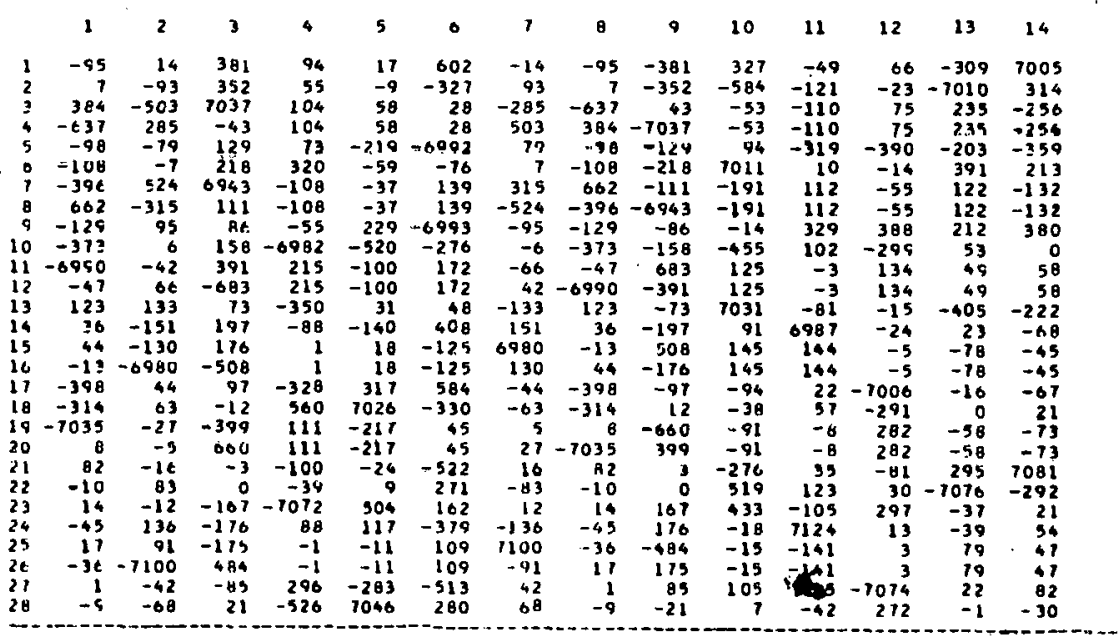


TABLE 33. GECMEITY DAC LCAO-MO'S FOR S-TRANS-ISOPROPYL ETHYLENE

$\begin{array}{ll}1 & C \\ 2 & C \\ 3 & C \\ 4 & C \\ 5 & C \\ 6 & H \\ 1 & H \\ 8 & H \\ 9 & H \\ 10 & H \\ 11 & H \\ 12 & H \\ 13 & H \\ 14 & H \\ 15 & H\end{array}$

$\begin{array}{ll} & \\ 0.0 & 0.0 \\ 0.0 & 0.0 \\ -1.316358184 & -0.000000001 \\ -1.397936410 & 1.257404704 \\ -1.397936407 & -1.257404703 \\ 0.935307370 & 0.0 \\ -0.935307371 & 0.0 \\ 0.935307371 & 0.000000000 \\ -2.144844400 & -0.000000001 \\ -1.340196021 & 2.147385323 \\ -0.565450195 & 1.257404706 \\ -2.341903406 & 1.257404703 \\ -2.341903404 & -1.257404704 \\ -0.569450192 & -1.257404702 \\ -1.340196015 & -2.147385323\end{array}$

0.0739599199

2.09998951

2.985367965

-0.539999962
-0.539999962

1.879999161

1.391684866
2.358711118

3.693682048

3.530307562

3.693682050

EXPANSICNS IN SLATER BASIS

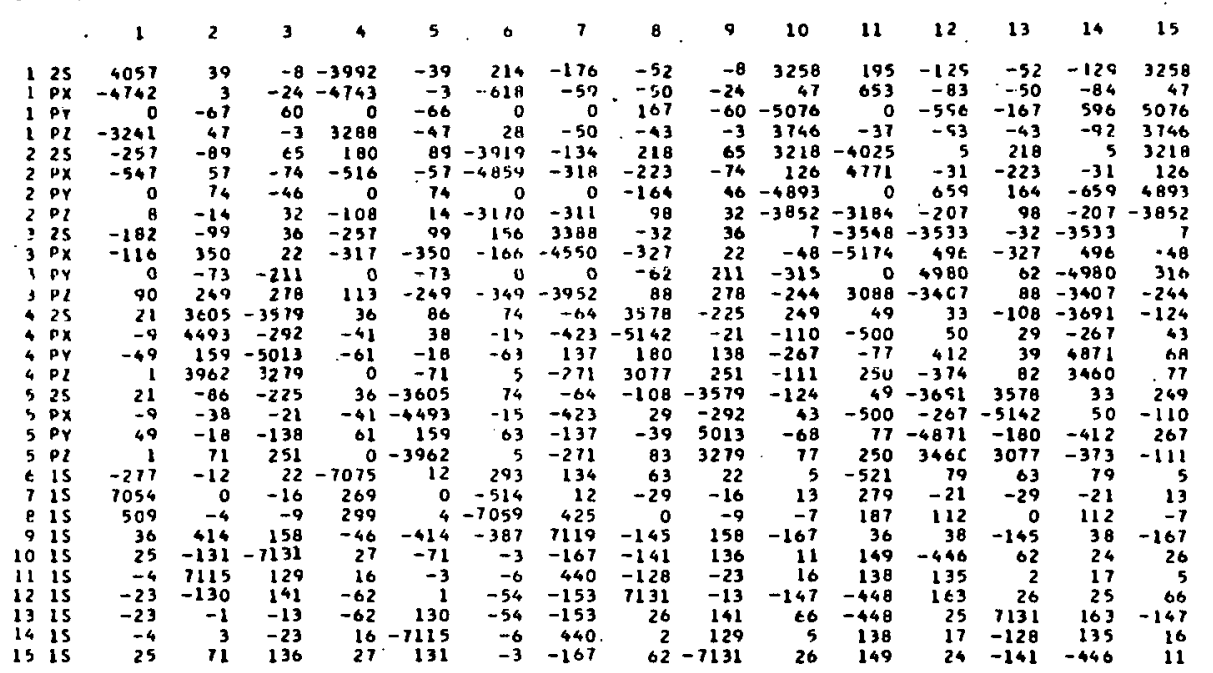

EXPANSICNS IN HYBRIO EASIS

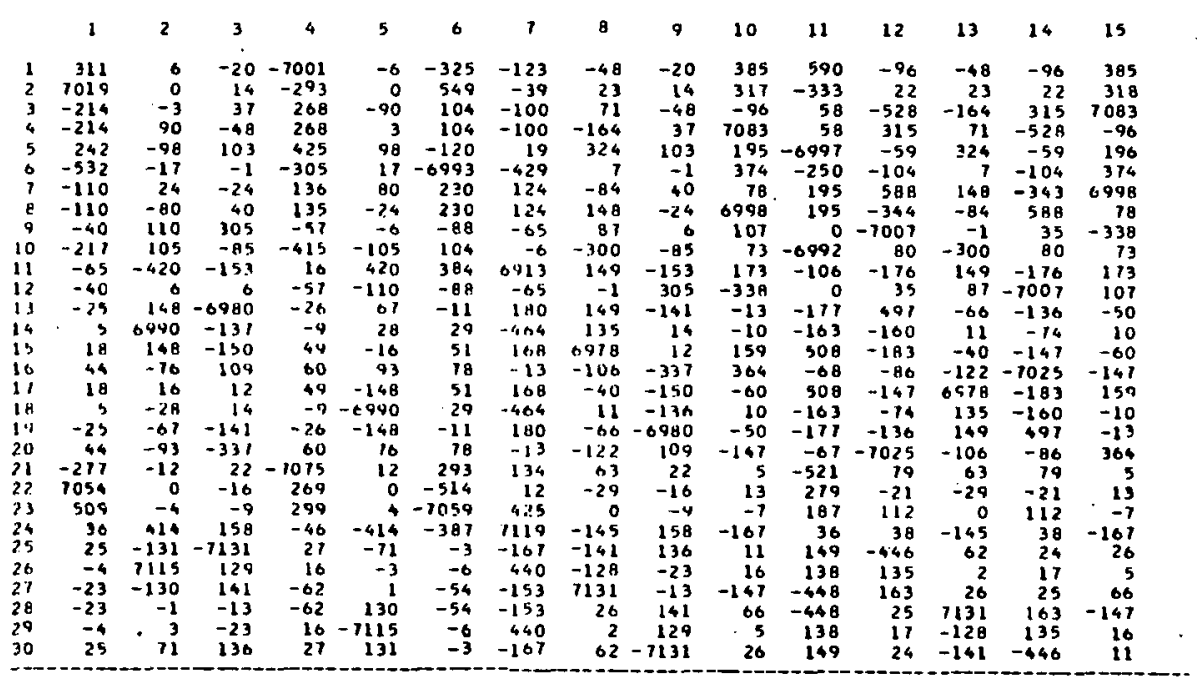


TAQLE 34. ANGLES IN DEgREES ANO BONDLENGTHS IN ANGSTROMS ASSIGNED BY THE MCOEL-BUILDER TO ACYCLIC PARAFFINS TABL 340 ANGLES IN OEGRES ANO BONDLENGTHS IN ANGSTRQMS ASSIGNED

$\begin{array}{llcc}\text { BOND } & \text { CH } & \text { SINGLE CC BONDS } & \text { DOUBLE TRIPLE } \\ \text { ANGLE BOND } & \text { TETRAHEDRAL TRIGONAL LINEAR BOND } & \text { BOND }\end{array}$

\begin{tabular}{|c|c|c|c|c|c|c|}
\hline $\begin{array}{l}\text { TETRAHEORAL } \\
\text { TRIGONAL } \\
\text { LINEAR }\end{array}$ & $\begin{array}{l}109.5 \\
120 \\
180\end{array}$ & $\begin{array}{l}1.09 \\
1.08 \\
1.06\end{array}$ & $\begin{array}{l}1.54 \\
1.52 \\
1.46\end{array}$ & $\begin{array}{l}1.52 \\
1.46 \\
1.45\end{array}$ & $\begin{array}{l}1.45 \\
1.45 \\
1.38\end{array} \quad 1.34$ & 1.20 \\
\hline
\end{tabular}


where (s), (x), etc. refer to the $2 s, 2 p x$, etc. AO's on carbon. Since there are four parameters, but only three constraints (normalization is automatic)

$$
\begin{gathered}
\langle g(+) \mid g(-)\rangle=0 \\
\langle h(+) \mid h(-)\rangle=0 \\
\langle g(t) \mid h(t)\rangle=0=\cos Q \cos R+\sin Q \operatorname{sinR} \cos A \cos B .
\end{gathered}
$$

There is one free parameter and one additional constraint may be imposed. The expicit relations that result from Equations 8-12 for the parameters Q, R, A, B are conveniently displayed as

$$
\begin{aligned}
& (\sin Q \sin A)^{2}=\frac{1}{2}, \\
& (\sin R \sin B)^{2}=\frac{1}{2}, \\
& \cos ^{2} Q+\cos ^{2} R=\frac{1}{2}
\end{aligned}
$$

and

$$
\cot ^{2} A+\cot ^{2} B=1
$$

Equations 13 and 14 relate the s-character to the angle in the same hybrid, Equation 15 connects their relative s-characters, and Equation 16 connects their opening angles. With these equations, it is straightforward to adjust the two hybrid pairs to various specific situations.

Tetrahedral carbon (four single bonds)

A first set of hybrids obtains by using the additional constraint to $\mathrm{fix}$ the angle between all centroids at $109.5^{\circ}$ (the tetrahedral value), i.e. 
by setting

$$
A=B \text {, }
$$

and from Equation 16

$$
\cos A=\sqrt{3} / 3
$$

The remaining parameters follow from Equations 13-14, so

$$
\begin{aligned}
& g( \pm)=.5(s)+.5(z) \pm .7071(x), \\
& h( \pm)=.5(s)-.5(z) \mp .7071(y) .
\end{aligned}
$$

These are the well-known $s p^{3}$ hybrids.

Irigonal carbon (two single bonds and one double bond)

Unlike the tetrahedral, the $g^{\prime} s$ and $h$ 's are inequivalent here.

Suppose the g's point in the direction of the single bonds and the $h$ 's are the bent banana hybrids pointing into the double bond. Thus

$$
A=60^{\circ}
$$

which implies that

$$
B=101^{\circ}
$$

giving

$$
g( \pm)=.5773(s)+.4082(z) \pm .7071(x)
$$

and

$$
h( \pm)=.4082(s)-.5773(z) \mp .7071(y) .
$$

The $g^{\prime}$ 's are the usual $s p^{2}$ hybrids, while the $h^{\prime} s$ are $s p^{5}$, with centroids $50^{\circ}$ above and below the bond skeleton. The g's, being single bond hybrids, are always coplanar with vicinal single bond neighbors. This forces the 
centroids of the h's to lie about five degrees away from exactly cis or trans positions.

Ethynyl Carbon (Single Bond and Triple Bond)

Construction of the hybrids

Let $p, p(i), i=1,2,3$ be four normalized linear combinations of $p$ orbitals satisfying

$$
\langle p \mid p(i)\rangle=0
$$

and

$$
\langle p(i) \mid p(j)\rangle=-\frac{1}{2}, i \neq j
$$

i.e., the $p(i)$ lie separated by $120^{\circ}$ in a plane orthogonal to $p$. From $p$ and the $2 \mathrm{~s}$ AO can be formed 'left' and 'right' hybrids which point directly at the neighbor atoms

$$
\begin{aligned}
& l=(s) \cos Q-(p) \sin Q \\
& r=(s) \sin Q-(p) \cos Q .
\end{aligned}
$$

The orthogonal, trigonal banana hybrids are formed from the $r$ and the $p(i)$

$$
t(i)=(r+p(i) \sqrt{2}) \sqrt{3} / 3
$$

They point into the triple bond and are orthogonal to the hybrid $l$ that points into the single bond. Since all of these hybrids are automatically orthogonal, $Q$ is a free parameter. It can therefore be adjusted such that the hybrid $\ell$ fits as closely as possible the atomic $s$ and $p$ contributions to the actual Inralized Mo's representing the single bonds next to triple 
bonds. The thirteen cases considered in this study gave

$$
\ell=.6736(s)-.7391(p)
$$

with deviations the same as those for the other single bonds $\left(s p^{3}\right.$ and $\left.s p^{2}\right)$. Note that $l$ is not an exact sp hybrid.

The expression for $\ell$ implies the expression

$$
t(i)=.4267(s)+.3889(p)+.5773 p(i)
$$

for the banana hybrids pointing into the triple bond. These are approximately $\mathrm{sp}^{5}$, and form an angle of about $63^{\circ}$ with the bond axis. It is striking that these hybrids form almost the same angle with each other as those pointing into the double bonds, namely $101^{\circ}$.

\section{Absolute orientation}

Since the ultimate goal is to recognize the similarities among localized orbitals, the free rotation of the $t(i)$ should be removed as consistently as possible. The method chosen was to maximize the overlap between a $t(i)$ and its contribution to one of the banana bond LMO's. Suppose $\ell$ and $r$ lie along the z-axis, and let the contributions from the perpendicular (xy) plane to the three LMO's in the triple bond be given by

$$
L(i)=a(i) \cdot(x)+b(i) \cdot(y), i=1,2,3 .
$$

The pair of Equations 25,26 can be expressed as

$$
p(i)=-(x) \sin (W+(i-1) \pi / 3)+(y) \cos (W+(i-1) \pi / 3)
$$


and the variation in $W$ describes the rotation of the hybrid triple around the bond axis. Optimal fit of $p(i)$ to $L(i)$ is therefore obtained from the condition

$$
d / d W[\langle L(i) \mid P(i)\rangle]=0
$$

which yields an angle $W(i)$ given by

$$
\tan (w(i))=-a(i) / b(i) .
$$

Three values of $W(i)$ are obtained in this way, from which the weighted mean is formed

$$
W=\sum_{i} W(i)\langle L(i) \mid p(1)\rangle \sum_{i}\langle L(i) \mid p(i)\rangle
$$

The absolute orientation of the $p(i)$, and hence the $t(i)$, about the bond axis is then obtained by inserting this value of $W$ into Equation 33 .

Even with this procedure, the triple bonds presented situations not encountered with the others. Because of very slow localization and/or slight molecular asymmetry, their centroids were sometimes not coplanar with those of vicinal single bonds. In the discussion of fragments that will be given later, we have therefore extrapolated these exceptions to coplanar orientations. 


\section{ENERGY LOCALIZED MO'S}

\section{Introduction}

Energy localized MO's (LMO's) exploit the invariance of the MO equations to unitary transformations, as was pointed out by Fock (15). The LMO's were themselves proposed later by Lennard-Jones and Pople (81), but remained largely conceptual, even after the advent of computers, unti $i$ the algorithm of Edmiston and Ruedenberg $(5,6)$ allowed their practical determination. In two papers including some diatomic and small polyatomic localizations $(6,7)$, the authors convincingly demonstrated that these were indeed rigorous quantum mechanical analogs to the bonds and lone pairs of chemical intuition, as had been hoped all along. Various interpretations of LMO's are reviewed in the Appendix.

\section{Localization Equations}

At first, the formal discussion of localized orbitals was confined to equivalent MO's, those which carry the regular representation (82) of the symmetry group, i.e., they are permuted among each other by the group operations. In passing, it was also mentioned that they presumably maxlmize the self-repulsion sum (localization sum)

$$
D=\Sigma_{u}\left[u^{2} \mid u^{2}\right]
$$

over all occupied MO's, where

$$
[u \mid v]=\iint d^{3} \underline{x} d^{3} \underline{z} u(\underline{x}) v(\underline{z}) /|\underline{x}-\underline{z}| \text {. }
$$

Edmiston and Ruedenberg (5) extended these ideas to general systems (i.e., 
the symmetry requirements necessary for equivalent orbitals were dropped) and adopted the maximization of $D$ and the resulting 'localization criteria'

$$
\left[u^{2}-v^{2} \mid u v\right]=0
$$

as the central features for the LMO determinations. This was done by series of successive $2 \times 2$ transformations $(5,6)$ rather like the jacobi diagonalization method (83). A remarkable feature of this method is that it has always converged as far as is known, even in those cases where it. has led to some ambiguities $(6,7)$. These are readily understood $(84)$.

\section{Semiempirical Energy Localization}

In principle, there are as many of these as there are semiempirical theories. Only several have appeared, however: a set of pi orbital localizations $(21,22)$ which used the Mulliken approximation (85) for electron repulsion integrals; and all valence electron localizations using CNDO (86) and INDO (63).

One might wonder what differences obtain between the last two approximations. It has been shown (63) that the retention of the one-center twoelectron integrals by INDO gives much better agreement with existing ab initio LMO's (INDO LMO's are much closer to the ab initio than to the CNDO: in particular, INDO reproduces the ab initio banana orbitals while CNDO does not mix the sigma and pi orbitals). Thus, semiempirical LMO's are sensitive to the method of approxlmating the electron repulsion integrals. This becomes especially important when topics such as hybridization are analyzed, and dictates the use of INDO for studying the paraffins. 
Starting Orbitals

The starting orbitals for the energy localization can be selected in. a way that is similar to that used for the Hückel orbitals (2I), and is possible for two reasons:

1. The canonical closed shell INDO MO's are readily available.

2. The LMO's in these paraffins are largely two-center 'bonds.' The procedure follows:

1. For each bond, form a perfectly homopolar two-center orbital $\left(x+x^{\prime}\right) \sqrt{2}$, where $x$ and $x^{\prime}$ are the two hybrids pointing into the bond.

2. Project each of these two-center orbitals into the space of the occupied canonical MO's.

3. Symmetrically orthogorialize these projections.

The details of this have been given. elsewhere (21). The starting localized MO's obtained this way turn out to be close approximations to the exact LMO's. This is shown in Table 35 for ethane, which lists the coefficients of the two ( $\mathrm{CH}$ and $\mathrm{CC}$ ) orbitals in the hybrid basis. The degree of approximation is typical.

First Order Localization

With starting orbitals as close to the LMO's as the SMO's, it is natural to use a first order scheme for each of the $2 \times 2$ localization steps discussed after Equation 39. Thus, if

$$
\begin{aligned}
& u_{i}=v_{i}+t_{i j} v_{j}, \\
& u_{j}=v_{j}+t_{j i} v_{i},
\end{aligned}
$$

with 
TABLE 35. CCMPARISON OF LOCALIZEO, LEAST MEAN SQUARE aNo PERTURBation mo's IN ETHANE

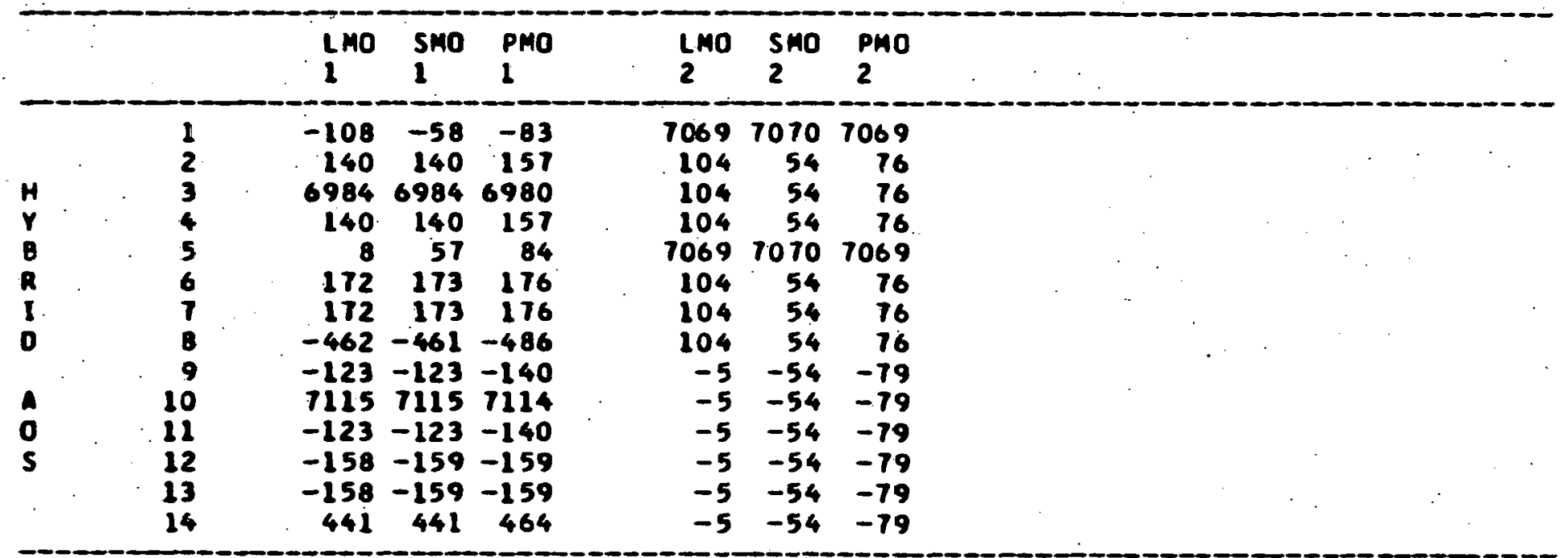




$$
t_{i j}=-t_{j i},
$$

then the u's are normalized to first order. Substitution of this expansion in Equation 39 yields for $t_{i j}$ the expression

$$
t_{i j}=[i i-j j \mid i j] /|4[i j \mid i j]-[i i-j j \mid i i-j j]|
$$

where all integrals are calculated with the MO's $v_{i}$. It leads to the following change in the localization sum $D$

$$
D^{\prime}=([i i-j j \mid i j])^{2} /[i j \mid i j]-[i i-j j \mid i i-j j] / 4 \mid \geq 0
$$

with each $i, j$ transformation. The procedure is applied to all distinct orbital pairs and iterated until the total change in $D$ is less than some predetermined criterion. Note that this method requires that only three kinds of repulsion integrals be calculated, which gives it a further advantage over the original algorithm (which requires the calculation of all types of repulsion integrals).

LMO's as Two-Center Orbitals Perturbed by Bond-Bond Interactions In the same context, it is of interest to compare a theory of Pople and Santry (87-89) with energy localization. These authors applied the perturbation theory of Coulson and Longuet-Higgins (90-93) to some simple paraffins by using a hybrid basis analogous to the present one and assuming that the unperturbed orbitals were two-center bonds. Only one-electron core energies and bond-bond interactions were included, and although the method strictly provides just the population matrix, coefficients can be extracted from it in several reasonable ways, all of which give about the 
same results. In order to make the comparison as favorable and simple as possible, the actual LMO's were truncated to two centers and used as the unperturbed functions with the appropriate INDO one-electron matrix elements.

The results (PMO's) for ethane are also shown in Table 35, and again are typical of the other molecules. The agreement with the LMO's is similar to that obtained for the SMO's and thus provides another interesting interpretation: LMO's can be thought of as arising from perturbed, perfectly two-center orbitals. This has two consequences:

1. It gives particular motivation to such concepts as 'delocalization' and 'overflow' which will be applied later.

2. The PMO's anticipate some of the dependences on relative bond orientations, types and distances that will play a great role in the LMO analysis.

Energy Localized Orbitals from Pseudo-Eigenvalue Equations

Ruedenberg (8) has shown that LMO's satisfy the pseudo-eigenvalue equations (see also the work of Gilbert (94), which is closely related)

$$
(F+L)|n\rangle=\gamma_{n n}|n\rangle, n=1, \ldots, N
$$

where $F$ is the Fock operator,

with

$$
L=\sum_{i \neq k}^{N} \sum_{i j}^{N}|i\rangle\left(c_{i k}-\gamma_{i k}\right)\langle k|
$$

$$
\gamma_{i k}=\langle i|F| k\rangle
$$

and

$$
c_{i k}=|\langle i i-k k \mid i k\rangle| \text {. }
$$


It was hoped that LMO's could be obtained with an SCF iteration process.

The SMO's provided an interesting chance to test this, being very close to the LMO's and thus presumably requiring few iterations for convergence. This was not the case however, for all attempts at iterative solution of Equation 46 failed: the $C_{i k}$, though small initially (for propane the largest were around .0025 a.u.)., did not become successively smaller.

The LCAO Expansions

The complete LCAO LMO's for each molecule are also presented in Tables 1-33. They are given in two bases, the INDO basis of Slater orbitals, and the previously described hybrid basis. The rows in the former are labelled by the AO type, preceded by the number of the atom centering it; and the rows in the latter are labelled by the numbers assigned the hybrids in Figure 1. The LMO's are also labelled by numbers and these form the column headings. The ordering of the molecules is the same as that in Figure 1. Further discussion of the LMO's will be in terms of the hybrid basis only and deferred until suitable moleculeindependent coefficients have been determined.

Localization was terminated when an iteration (each set of $N(N-1) / 2$ transformations) left D stable to ten decimals. It was found that even in the worst cases this gave coefficients stable to several parts in ten in the fourth decimal place. Due to the development of the first order method toward the end of the work, the usual Edmiston-Ruedenberg scheme was used, normally taking fifteen minutes or less on an IBM $360 / 50$, but needing more 
than thirty minutes in some of the larger or triply bonded molecules. The first order method is both faster and easier to program, but the impracticality of repeating calculations, machine changes, et.c., made a thorough comparison of relative times difficult. However, they improved by factors of two to five, even without taking full advantage of the fewer integral types required. Thus, the savings is certainly worthwhile. 


\section{MOLECULE INVARIANT LMO FRAGMENTS}

\section{Local Delocalization and LMO Fragments}

It is clear from the LCAO expansions in the hybrid basis (Tables 1-33) that the LMO's are not confined to two centers. This fact is related to the occurrence of long range bond orders, which was pointed out by Pople and Santry $(87,88)$, but seems not to have been widely appreciated, perhaps because such delocalization appears intuitively unappealing. However, recent studies from this laboratory have revealed the importance of such 'local delocalization' in many contexts: the origin of aromatic stability is due to geminal delocalization $(21,22)$, and the origins of rotation barriers $(18,19,69,70)$ as well as the systematic interpretation of bond dipole moments and energies $(20)$ is intimately connected with vicinal delocalization. There are therefore good reasons to devote an essential part of the present study to the delocalization of the LMO's.

Local delocalization means that each LMO contains contributions from various bond regions. In the present discussion, the term 'bond region' will be used to denote the space lying between two chemically bonded atoms. Each LMO is therefore a sum of several LMO fragments, each of which is associated with a specific bond region. The character of these LMO fragments will be the principal objective of the subsequent discussion because, in acyclic paraffins, transferability and regular behavior are most clearly revealed by them. Standard forms for the (LMO) fragments will be cataloged for various conditions in series of tables from which they can be retrieved to construct practically exact LMO's for almost any 
given acyclic hydrocarbon without solving any equations. For those few exceptions that are not covered by this work, close estimates (to within a few percent) can be obtained by sensible perusal of the discussion of related cases.

An LMO fragment is characterized (1) by the LMO to which it belongs and (2) by the bond region in which it is located. We shall discuss both in turn.

\section{LMO Characteristics of Fragments}

The LMO characteristics of a fragment are based on the fact that by far the largest part of each LMO lies in one particular bond region (the coefficients of the hybrids pointing into this region are always about 0.7), so that it is justifiable to say: a particular LMO 'essentially describes' the bond in which it is predominantly concentrated. Thus, LMO number three in pentane (Table 16) essentially describes the single bond between the atoms $C_{1}$ and $H_{6}$. This principal fragment will be called the bond fragment of that LMO, and it will be used to characterize the LMO itself. Thus the LMO number three will be called a ' $\mathrm{CH}$ bond LMO.'

All other fragments of an LMO will be characterized by their location relative to the bond fragment, denoted as geminal fragments, vicinal fragments and third and fourth neighbor fragments. Thus in LMO number three of pentane (Table 16), the fragment between atoms $c_{1}$ and $c_{2}$ is a geminal fragment, the one between $C_{2}$ and $C_{3}$ is a vicinal fragment, and those between $C_{3}$ and $C_{4}$ and $c_{4}$ and $c_{5}$ are third and fourth neighbor fragments, respectively. Higher fragments will not be considered because of their 
small coefficients.

We adopt the convention that the bond fragment is positive, which implies that, in each LMO, the coefficients of the hybrids in the bond fragment are positive.

Characterization of LMO Fragments by Bond Regions

The characterization of an LMO fragment with respect to the nature of the bond region In which it lies has to do with the location of that bond region within the total bond skeleton. The character of a bond region, in turn, is determined by the hybrid AO's pointing into it from the two constituent atoms: We start therefore by classifying the character of these hybrids. In the following the term 'hybrid' is always meant to include the hydrogen $A 0$, unless stated otherwise.

Hybrid AO's can be located on four types of atoms

(1) on hydrogen atoms denoted by $\mathrm{H}$;

(2) on alkyl type carbon atoms $(>c<)$;

(3) on vinyl type carbon atoms $(=C<)$ denoted by $C V$;

(4) on ethynyl type carbon atoms $\left(\mathrm{CC}^{-}\right)$denoted by CE.

The carbon atoms can be further distinguished as

(1) methanic (bonded to hydrogens only);

(2) primary (bonded to one other carbon), denoted by CP;

(3) secondary (bonded to two other carbons), denoted by CS;

(4) tertiary (bonded to three other carbons), denoted by CT;

(5) quaternary. (bonded to four other carbons), which will not occur in this investigation. 
Finally, the carbon hybrids will be characterized by what kind of bond lies in the coplanar positions(s) vicinal to them, cis or trans. These are denoted by

(1) $\mathrm{H}$, when a $\mathrm{CH}$ bond is vicinal;

(2) C, when a CC bond is vicinal;

(3) 2 , when a double bond is vicinal;

(4) 3, when a triple bond is vicinal.

The cis are distinguished from the trans by an asterisk, e.g. H* means the $\mathrm{CH}$ bond in the vicinal position lies cis.

Thus there exist a considerable number of different hybrid $A O$ 's and every bond region can be characterized by the hybrids pointing into it. Since these LMO fragment characteristics will occur again and again in the subsequent discussions, we shall give here a complete description for those that occur in the study, together with the abbreviations to be used in the sequel. Following this, we shall show how the cis and trans fragment abbreviations are used.

1. $\quad \mathrm{CPH}=$ single bond between primary alkyl carbon and hydrogen.

2. $\mathrm{CSH}=$ single bond between secondary alkyl carbon and hydrogen.

3. $C T H=$ single bond between tertiary alkyl carbon and hydrogen.

4. $\quad C P V H=$ single bond between primary vinyl carbon and hydrogen.

5. $\mathrm{CSVH}=$ single bond between secondary vinyl carbon and hydrogen.

6. $\mathrm{CEH}=$ single bond between primary ethynyl carbon and hydrogen.

7. $C P C P=$ single bond between two primary alkyl carbons.

8. CPCS $=$ single bond between primary and secondary alkyl carbons

9. CPCT $=$ single bond between primary and tertiary alkyl carbons. 
10. CPCSV = single bond between primary alkyl and secondary vinyl carbons.

11. CPCTV = single bond between primary alkyl and tertiary vinyl carbons.

12. $\quad$ CPCE = single bond between primary alkyl and secondary ethynyl carbons.

13. $\operatorname{CSCS}=$ single bond between two secondary alkyl carbons.

14. $\operatorname{CSCT}=$ single bond between secondary and tertiary alkyl carbons.

15. $\operatorname{cscs} v=$ single bond between secondary alkyl and secondary vinyl carbons.

16. CSCE = single bond between secondary alkyl and secondary ethynyl carbons.

17. $\operatorname{CTCSV}=$ single bond between tertiary alkyl and secondary vinyl carbons.

18. CTCE = single bond between tertiary alkyl and secondary ethynyl. carbons.

19. CSVCSV = single bond between two secondary vinyl carbons.

20. CSVCTV = single bond between secondary and tertiary vinyl carbons.

21. CSVCE $=$ single bond between secondary vinyl and secondary ethynyl carbons.

22. CECE = single bond between two secondary ethynyl carbons.

23. $B C P V C P V=$ one of the double banana bonds between two primary vinyl carbons.

24. $B C P V C S V=$ one of the double banana bonds between primary and secondary vinyl carbons. 
25. BCPVCTV = one of the double banana bonds between primary and tertiary vinyl carbons.

26. BCSVCSV = one of the double banana bonds between two secondary vinyl carbons.

27. $\mathrm{BCPECPE}=$ one of the triple banana bonds between two primary ethynyl carbons.

28. BCPECSE $=$ one of the triple banana bonds between primary and secondary ethynyl carbons.

29. $\mathrm{BCSECSE}=$ one of the triple banana bonds between two secondary ethynyl carbons.

We point out the symbols are 'symmetric,' i.e., CSCP means the same thing as CPCS. The trans fragments are described by adding symbols to these, usually preceded by a dash. For example, CPCT-H23 means that a $\mathrm{CH}$ bond, a double bond and a triple bond all lie trans to the CPCT bond. If the double bond lay cis, this would be described as CPCT-H2*3. Many times, the $\mathrm{CH}$ will be understood, e.g., the preceding will be written CPCT $-2 * 3$.

Finally, we distinguish in each LMO fragment the near hybrid and the far hybrid, according to their position relative to the bond fragment of. the LMO. 


\section{ORBITAL BOND FRAGMENTS}

\section{General Properties}

The molecule invariant (Mi) bond fragments are the only fragments for which the coefficients of both constituent hybrids are positive. Furthermore, all are fairly homopolar with coefficients near 0.7 , an order of magnitude greater than those in other fragments.

\section{Geminal influences}

The slight polarities of the single bonds follow a general pattern that can be summarized by stating that the coefficients on various types of atoms satisfy the following inequalities

$$
C E>H>C P>C S \approx C T \approx C S V \approx C T V .
$$

and

$$
\text { H > CPV. }
$$

Similarly, the coefficients on banana hybrids in double and triple banana bonds satisfy the inequalities

$$
\text { CPV > CSV > CTV }
$$

and

$$
\text { CPE > CSE, }
$$

respectively. In comparing fragments with different geminal character, e.g. $P, S$ and $T$ ) and identical vicinal fragments, we shall use the term corresponding fragments. 
Vicinal influences

Finer differences between bond fragments within the gross behavior just described are induced by the coplanar vicinal fragments of the same LMO. In particular:

1. If bond and coplanar vicinal fragments are separated by a single bond, the type and orientation of the vicinal fragments usually influences the bond coefficients (in cases where higher fragments come into proximity with the bond fragment, the geometry of the higher fragments has some effect, too).

2. If bond and coplanar vicinal fragments are separated by a double bond; the geometry of the vicinal and higher fragments affects the bond coefficients.

3. If bond and coplanar vicinal fragment are separated by a triple bond, the vicinal fragment has little influence.

The $\mathrm{CH}$ bonds are least sensitive to vicinal influences, followed, in order, by $C C$ and double and triple banana bonds. To a good approximation, the influences of several vicinal bonds are simply additive. When comparing bond fragments with the same geminal character, but different vicinal elements, we frequently call the vicinal fragments substituents.

\section{Alkyl CH Bonds}

The various types of alkyl $\mathrm{CH}$ bond fragments appear in the first two rows of Tables 36-39. The first row contains the carbon sp ${ }^{3}$ contributions and the second now lists the hydrogen is contributions. The E's and V's in parentheses refer to positions of ethynyl and vinyl carbons, respectively, 
TABLE 36. BOND AND GEMINAL FRAGMENTS IN METHANE AND PRIMARY CH LMD'S

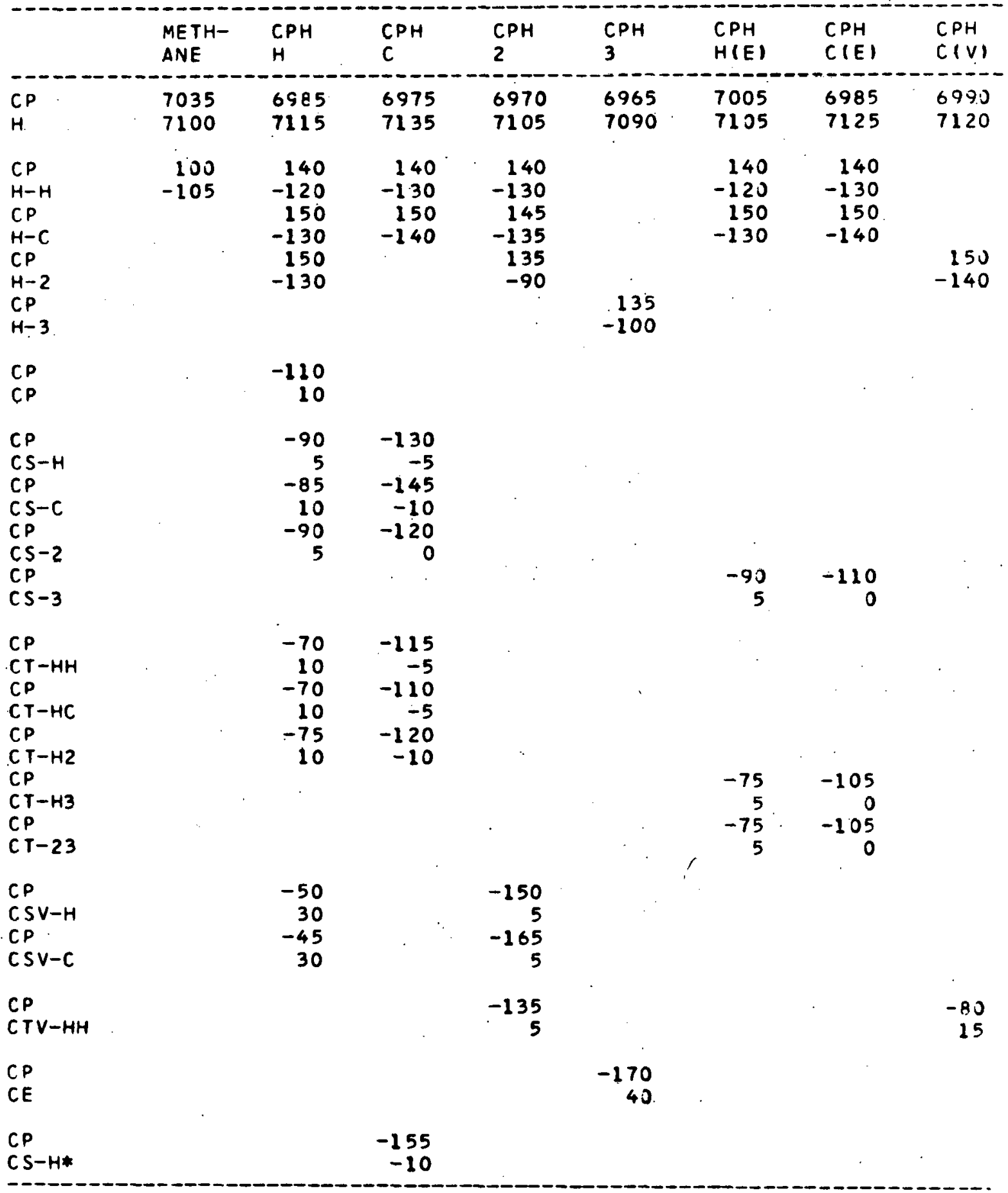


TABLE 37. BOND AND GEMINAL FRAGMENTS IN SECONDARY CH LMO'S

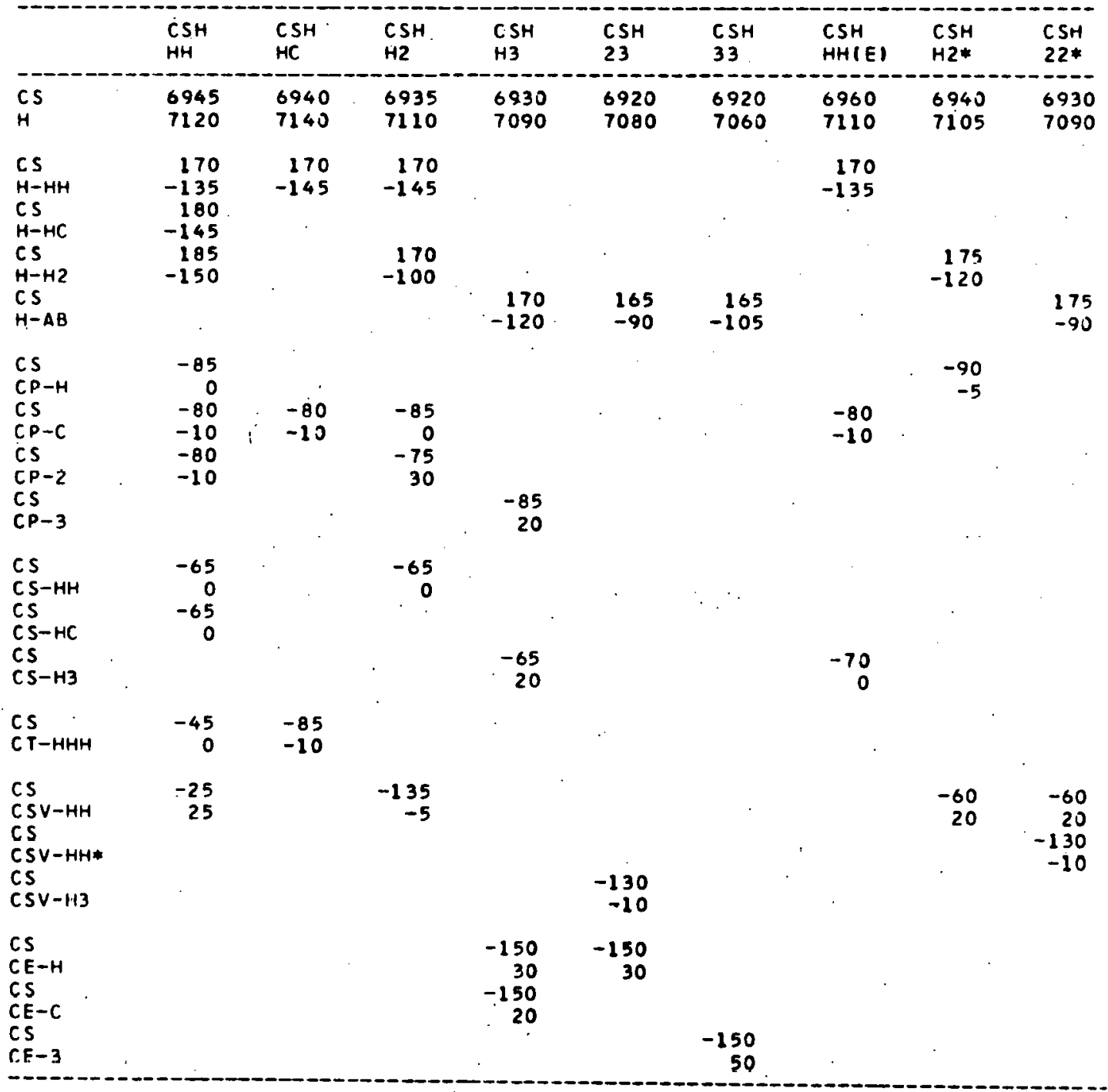


TABLE 38. BOND AND GEMINAL FRAGMENTS IN TERTIARY CH. LMO'S

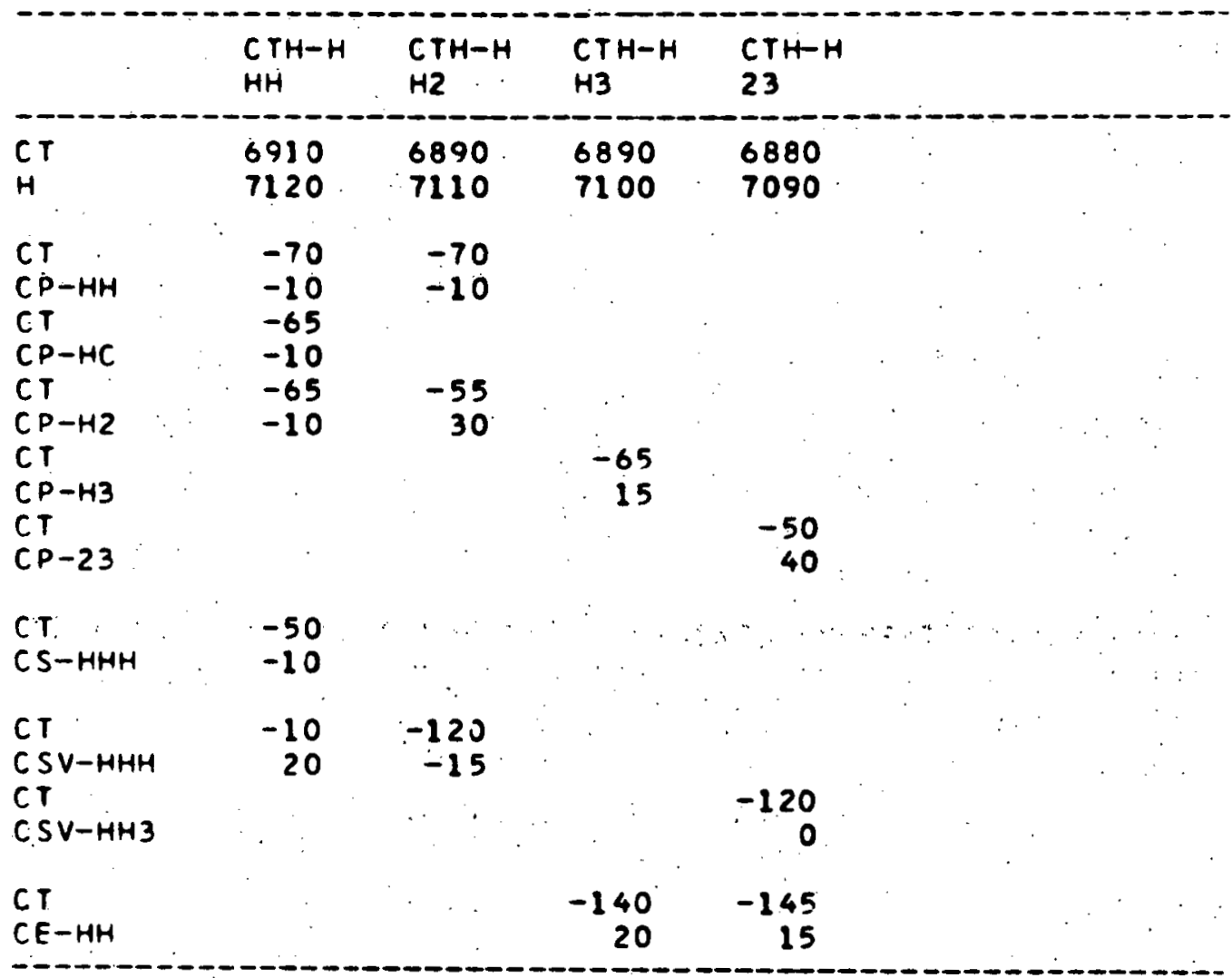


TABLE 39. BOND AND GEMINAL FRAGMENTS IN CIS PRIMARY CH LMO'S

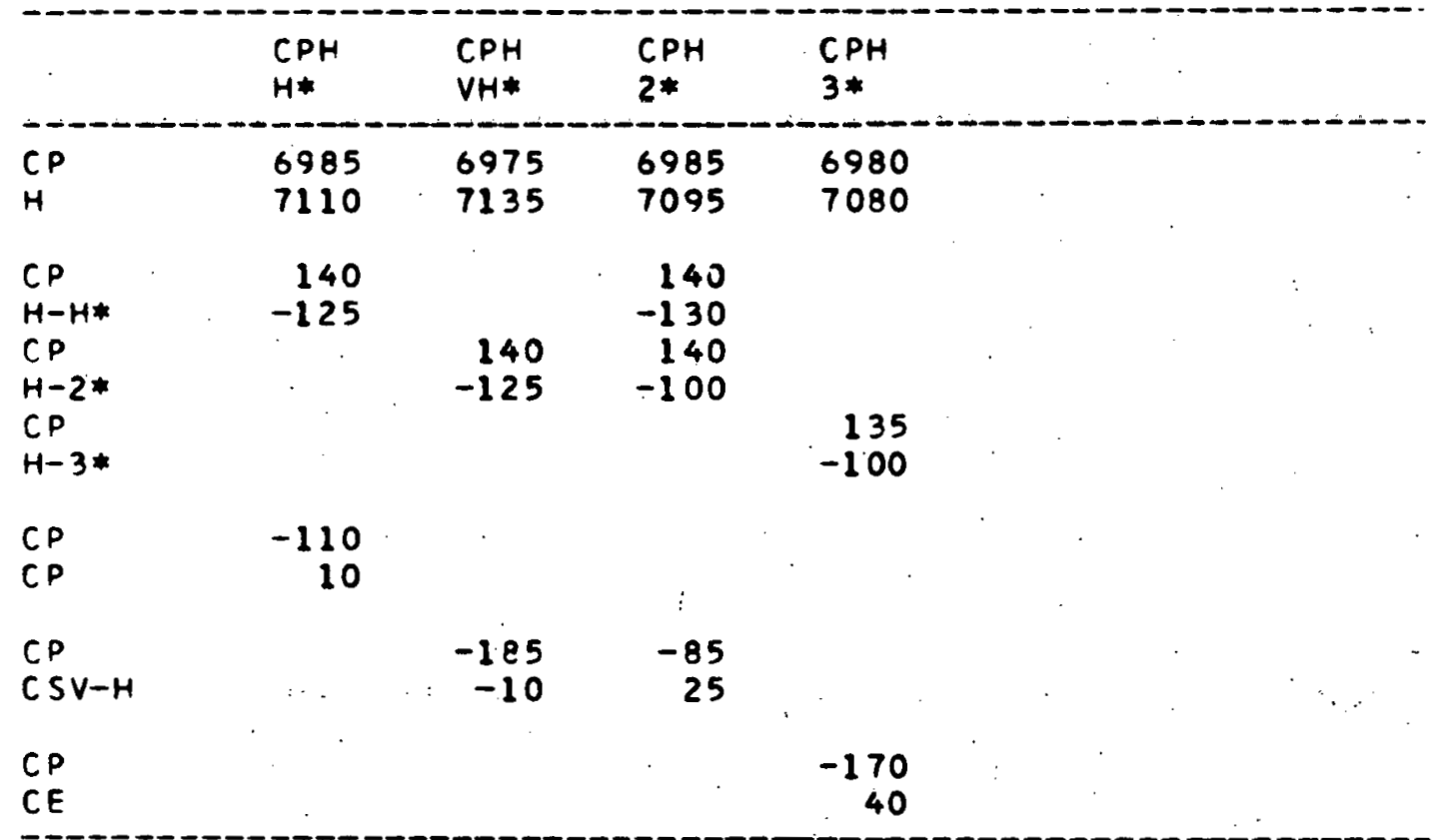


which are defined in Figure 2 . The VH* in Table 39 distinguishes the cases for which vinyl $\mathrm{CH}$ bonds occur cis to primary $\mathrm{CH}$ bond fragments...

\section{Polarity}

Overall, the fragments are very similar. Except for methane, all bond fragments display a is coefficient greater by about $2 \%$, and thus the Mulliken gross atomic populations on the hydrogens are always about $4 \%$ larger than those on the carbons.

\section{Geminal effects}

The geminal effects are seen in that the coefficients for the $s p^{3}$ in corresponding fragments, e.g. CPH-2, CSH-2 and CTH-2, decrease by about 40 with each step in the series $\mathrm{CPH}$ to $\mathrm{CSH}$ to $\mathrm{CTH}$, while the is coefficients are almost constant.

\section{Vicinal effects}

Vicinal effects can be assessed by using the CPH-H, CSH-HH and CTH-HHH as prototypes and considering the $\mathrm{CC}, 2,3$, etc. fragments as substituents. The effects of these 'substitutions' appear in Table 40. The numbers tabulated are those which must be added to the prototype fragment to obtain the substituted fragment, e.g. -10 and 20 must be added to the sp ${ }^{3}$ and is coefficients of $\mathrm{CPH}-\mathrm{H}$, respectively, to obtain those of $\mathrm{CPH}-\mathrm{C}$. Inspection of the table reveals that, to a very good approximation, the substituent effects are the same for each of the a.lkyl CH types. Moreover, according to the $\mathrm{CH}-23$ and $\mathrm{CH}-33$ rows, the effects of several vicinal substituents are simply additive. 
Figure 2. Bond skeleton and relative orientation nomenclatures 


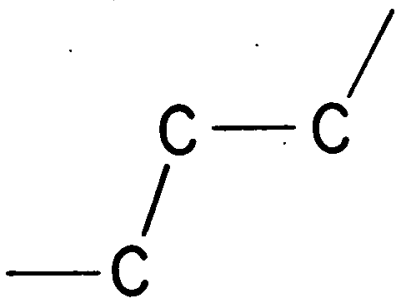

TT-BOND SKELETON

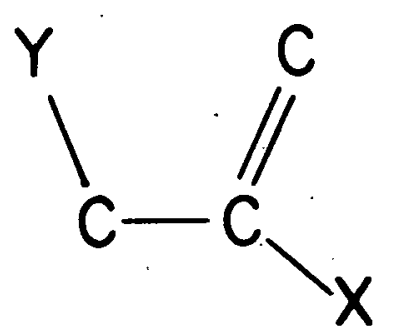

CY

$X(V)$

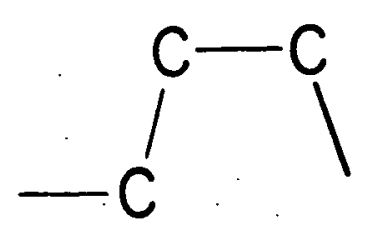

TC-BOND SKELETON

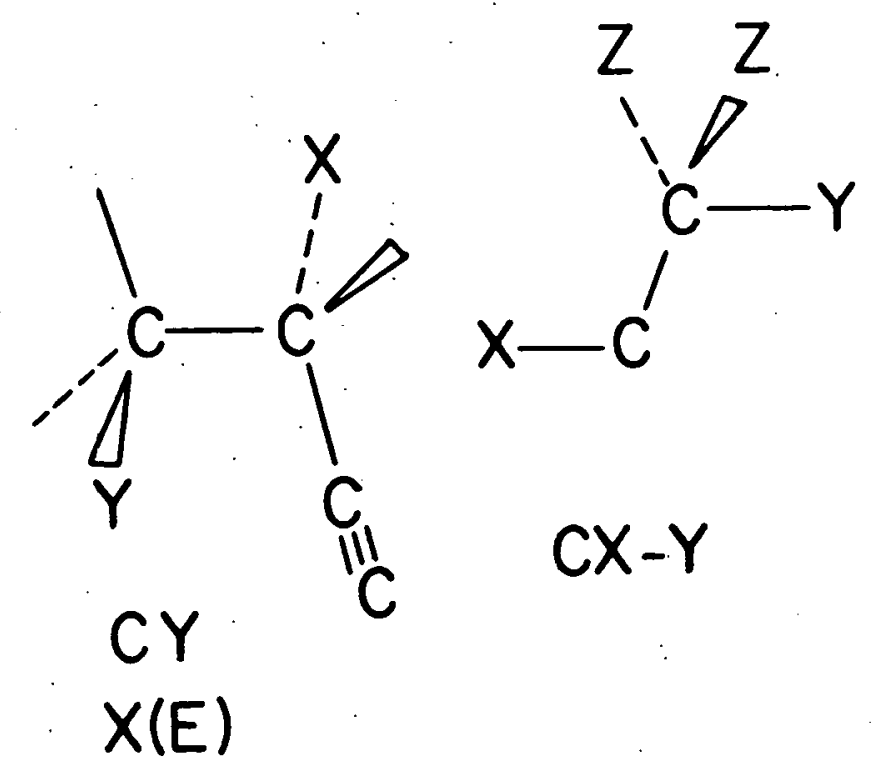

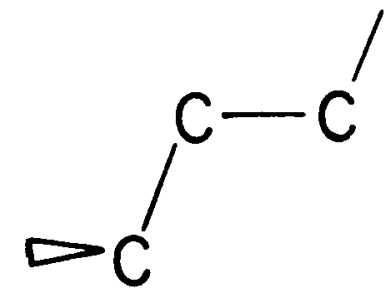

GT - BOND SKELETON SKELETON

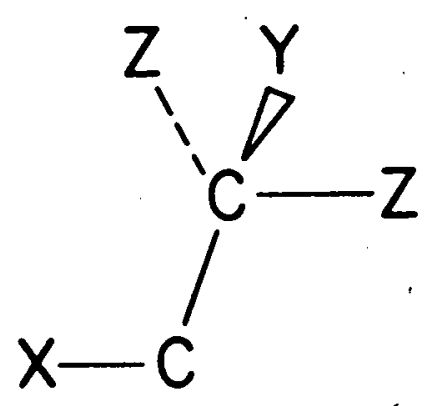

$C X-Z A$ 


\begin{tabular}{|c|c|c|c|c|c|}
\hline & $\mathrm{CPH}$ & $\mathrm{CSH}$ & CTH & CSVH & \\
\hline $\begin{array}{l}\mathrm{C} \\
\mathrm{H}-\mathrm{C}\end{array}$ & $\begin{array}{r}-10 \\
20\end{array}$ & $\begin{array}{l}-5 \\
20\end{array}$ & & $\begin{array}{r}-10 \\
20\end{array}$ & \\
\hline $\begin{array}{l}\mathrm{C} \\
\mathrm{H}-2\end{array}$ & $\begin{array}{l}-15 \\
-10\end{array}$ & $\begin{array}{l}-10 \\
-10\end{array}$ & $\begin{array}{l}-20 \\
-10\end{array}$ & & \\
\hline $\begin{array}{l}\mathrm{C} \\
\mathrm{H}-3\end{array}$ & $\begin{array}{l}-20 \\
-25\end{array}$ & $\begin{array}{l}-15 \\
-30\end{array}$ & $\begin{array}{l}-20 \\
-20\end{array}$ & . & : \\
\hline $\mathrm{C}-23$ & & $\begin{array}{l}-25 \\
-40\end{array}$ & $\begin{array}{l}-30 \\
-30\end{array}$ & . & \\
\hline $\begin{array}{l}\mathrm{C} \\
\mathrm{H}-33\end{array}$ & & $\begin{array}{l}-25 \\
-60\end{array}$ & & & . \\
\hline $\begin{array}{l}C \\
H-H(E)\end{array}$ & $\begin{array}{r}20 \\
-10\end{array}$ & $\begin{array}{r}15 \\
-10\end{array}$ & & & . \\
\hline $\begin{array}{l}C \\
H-C(E)\end{array}$ & $\begin{array}{r}0 \\
10\end{array}$ & . & & $\begin{array}{l}-5 \\
10\end{array}$ & 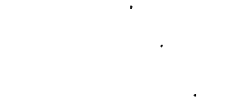 \\
\hline$C_{H-C(v)}^{C}$ & $\begin{array}{l}5 \\
5\end{array}$ & & & & \\
\hline $\begin{array}{l}\mathrm{C} \\
\mathrm{H}-\mathrm{H} *\end{array}$ & $\begin{array}{r}0 \\
-5\end{array}$ & & & $\begin{array}{r}-5 \\
0\end{array}$ & \\
\hline $\begin{array}{l}C \\
H-V H *\end{array}$ & $\begin{array}{r}-10 \\
20\end{array}$ & & & & \\
\hline $\begin{array}{l}\mathrm{C} \\
\mathrm{H}-\mathrm{C} *\end{array}$ & & . & & $\begin{array}{r}-5 \\
10\end{array}$ & \\
\hline $\begin{array}{l}\mathrm{C} \\
\mathrm{H}-2 *\end{array}$ & $\begin{array}{r}0 \\
-15\end{array}$ & $\begin{array}{r}-5 \\
-15\end{array}$ & & & \\
\hline $\begin{array}{l}\mathrm{C} \\
\mathrm{H}-3 \text { - }\end{array}$ & $\begin{array}{r}-5 \\
-30\end{array}$ & V & & $\begin{array}{l}-15 \\
-20\end{array}$ & \\
\hline $\begin{array}{l}\mathrm{C} \\
\mathrm{H}-22 \%\end{array}$ & & $\begin{array}{l}-15 \\
-30\end{array}$ & & & \\
\hline
\end{tabular}


Alkyl CC Bonds

Polarity

These are the. $\mathrm{CC}$ analogs to the alkyl $\mathrm{CH}$, the $\mathrm{H}$ being replaced by an alkyl, vinyl or ethynyl carbon. They appear in Tables 41-45 (in Table 41. they are listed in the first two columns, in the remaining tables in the first two rows). The coefficients for a particular fragment are in general more nearly equal than was the case for the alkyl $\mathrm{CH}$, and are slightly larger than the carbon $s p^{3}$ coefficients (in the $\mathrm{CH}$ ). Usually the magnitudes of the coefficients satisfy

$$
\mathrm{CE}>\mathrm{CP}>\mathrm{CSV} \approx \mathrm{CTV}>\mathrm{CS} \approx \mathrm{CT} \text {. }
$$

But when an ethynyl carbon neighbors the fragment, and if the fragment itself contains no ethynyl carbon, the coefficient on the carbon next to the ethynyl becomes largest.

\section{Geminal substitution}

There is a difference between the $\mathrm{CC}$ and $\mathrm{CH}$ alkyl bond fragments here, for if one passes, say, from the CPCP to the CSCP to the CTCP, all coefficients change, while only the $s P^{3}$ coefficients changed in the series.CPH, CSH and CTH. That is, both coefficients change with geminal substitution in the alkyl CC fragments.

vicinal substitution

These effects are given in Table 46. The prototype fragments are the $C X C P-H, C X C X-H H, C X C X V-H H$ and $C X C E-H$, where $X$ is $S$ or $T$. The regularity 
TABLE 41. BOND AND GEMINAL FRAGMENTS IN SATURATED PRIMARY CC LMO'S

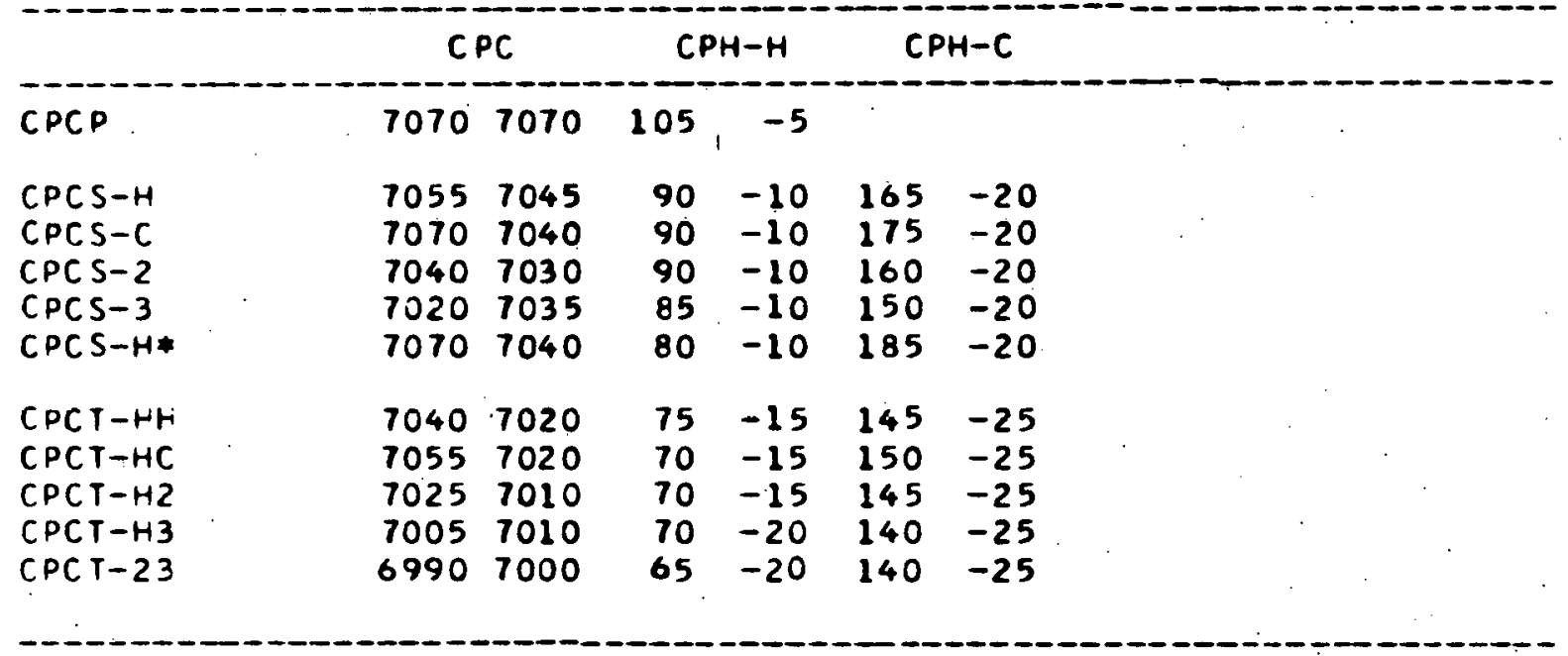




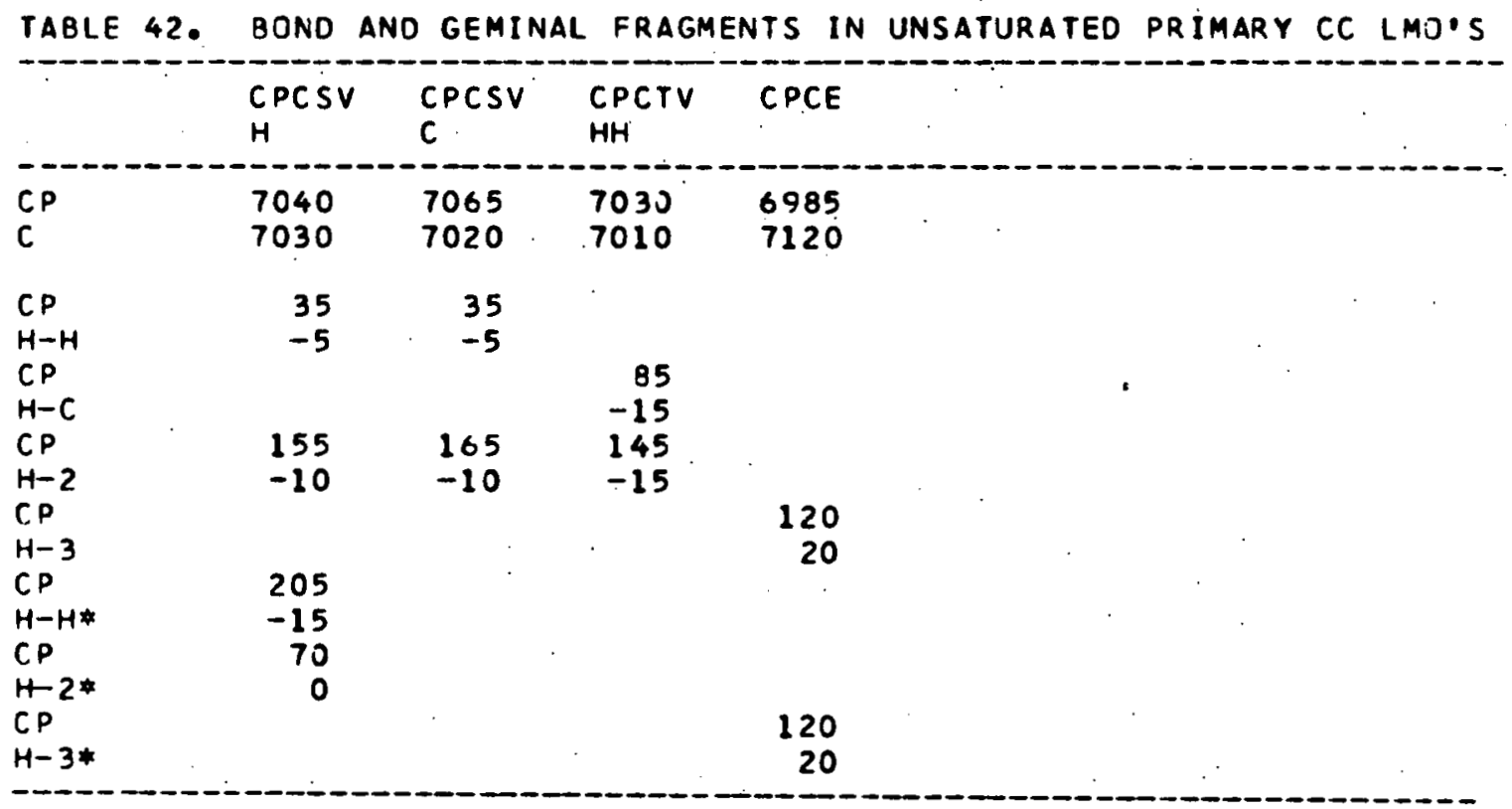


TABLE 43. BOND ANO GEMINAL FRAGMENTS IN SATURATED SECUNDARY CC LMO'S

\begin{tabular}{|c|c|c|c|c|c|c|c|c|c|}
\hline . & $\begin{array}{l}\text { CSCP } \\
H\end{array}$ & ${ }_{C}^{C S C P}$ & $\begin{array}{l}\operatorname{CSC} p \\
2\end{array}$ & $\begin{array}{l}\operatorname{CSC} P \\
3\end{array}$ & $\begin{array}{l}\operatorname{CsCP} \\
H^{*}\end{array}$ & $\begin{array}{l}\operatorname{cscs} \\
\mathrm{H}\end{array}$ & $\begin{array}{l}C S C S \\
H C\end{array}$ & $\begin{array}{l}\text { C.SCS } \\
\mathrm{H}_{3}\end{array}$ & $\begin{array}{l}\mathrm{CSCT} \\
\mathrm{HHH}\end{array}$ \\
\hline $\begin{array}{l}\text { CS } \\
c\end{array}$ & $\begin{array}{l}7045 \\
7055\end{array}$ & $\begin{array}{l}7040 \\
7.070\end{array}$ & $\begin{array}{l}7030 \\
7040\end{array}$ & $\begin{array}{l}7035 \\
7020\end{array}$ & $\begin{array}{l}7040 \\
7070\end{array}$ & $\begin{array}{l}7030 \\
7030\end{array}$ & $\begin{array}{l}7030 \\
7045\end{array}$ & $\begin{array}{l}7025 \\
6990\end{array}$ & $\begin{array}{l}7010 \\
70 ! J\end{array}$ \\
\hline $\begin{array}{l}\mathrm{CS} \\
\mathrm{H}-\mathrm{HH} \\
\mathrm{CS} \\
\mathrm{H}-\mathrm{HC}\end{array}$ & $\begin{array}{r}140 \\
-25\end{array}$ & $\begin{array}{l}140 \\
-30 \\
145 \\
-35\end{array}$ & $\begin{array}{l}145 \\
-25\end{array}$ & & & $\begin{array}{r}125 \\
-25\end{array}$ & $\begin{array}{l}125 \\
-30\end{array}$ & $\begin{array}{r}120 \\
-30\end{array}$ & $\begin{array}{l}110 \\
-35 \\
175 \\
-40\end{array}$ \\
\hline $\begin{array}{l}\mathrm{CS} \\
\mathrm{H}-\mathrm{H}_{2} \\
\mathrm{CS} \\
\mathrm{H}-\mathrm{H}_{3}\end{array}$ & $\begin{array}{r}145 \\
-35\end{array}$ & & $\begin{array}{r}130 \\
5\end{array}$ & $\begin{array}{r}130 \\
0\end{array}$ & $\begin{array}{l}140 \\
-20\end{array}$ & $\begin{array}{l}130 \\
-40\end{array}$ & . & $\begin{array}{l}120 \\
-10\end{array}$ & \\
\hline $\begin{array}{l}C S \\
C P-H\end{array}$ & $\begin{array}{r}-60 \\
65\end{array}$ & & & & & & & & \\
\hline $\begin{array}{l}C S \\
C P-C\end{array}$ & & . & & & & $\begin{array}{r}-15 \\
55\end{array}$ & $\begin{array}{l}-5 \\
60\end{array}$ & $\begin{array}{r}-20 \\
60\end{array}$ & $\begin{array}{r}-30 \\
55\end{array}$ \\
\hline $\begin{array}{l}\text { CS } \\
\text { CS }-H H \\
\text { CS } \\
\text { CS }-H C \\
\text { CS } \\
\text { CS }-H_{3}\end{array}$ & . & $\begin{array}{r}-85 \\
55 \\
-95 \\
50 \\
-65 \\
60\end{array}$ & & & & . & $\begin{array}{r}-40 \\
60\end{array}$ & & \\
\hline $\begin{array}{l}\text { CS } \\
\text { CSV-HH } \\
\text { CS } \\
\text { CSV-HC }\end{array}$ & $\begin{array}{r}-15 \\
90\end{array}$ & & $\begin{array}{r}-95 \\
70\end{array}$ & & $\begin{array}{r}-135 \\
50\end{array}$ & $\begin{array}{l}30 \\
80\end{array}$ & & & . \\
\hline $\begin{array}{l}C S \\
C E-H \\
C S \\
C E-C\end{array}$ & & & & $\begin{array}{r}-115 \\
115\end{array}$ & . & . & & $\begin{array}{l}-70 \\
110\end{array}$ & . \\
\hline
\end{tabular}


TABLE 44. BOND ANO GEMINAL FRAGMENTS IN UNSATURATEO SECONOARY CC LMO'S

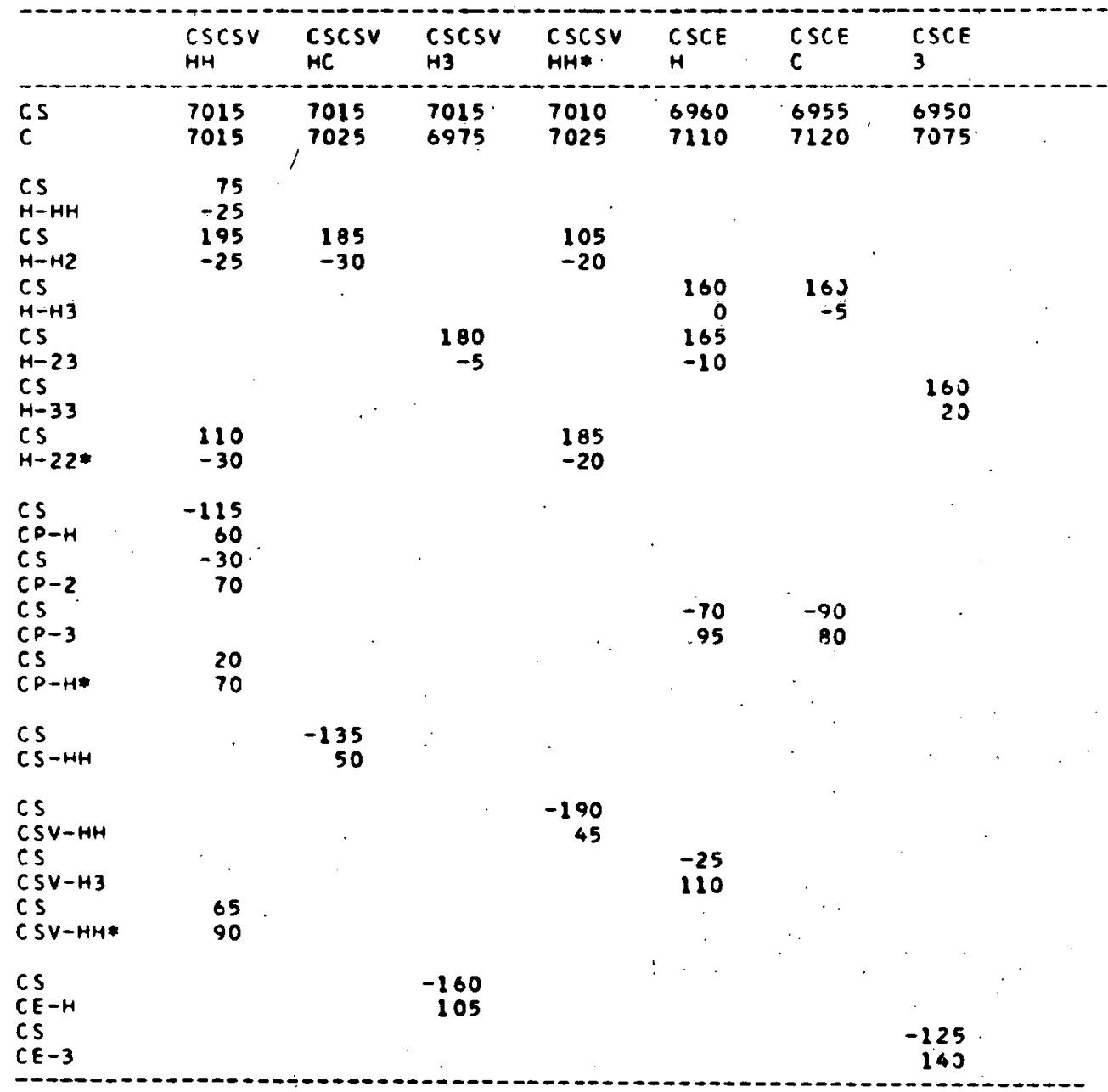




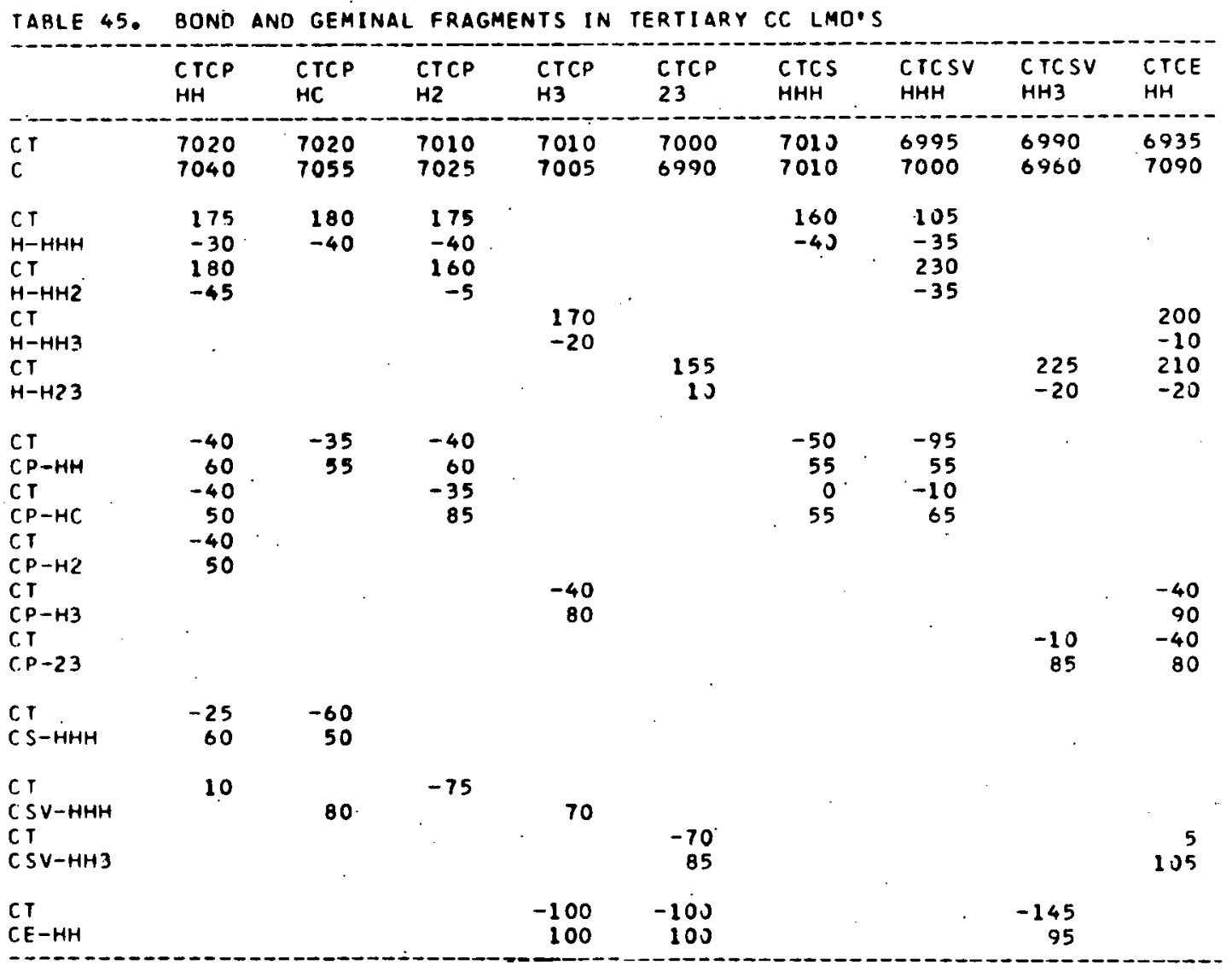


TABLE 46. SUBSTITUENT EFFECTS ON BOND FRAGMENTS IN CC ANC DOUBLE BANANA BONOS

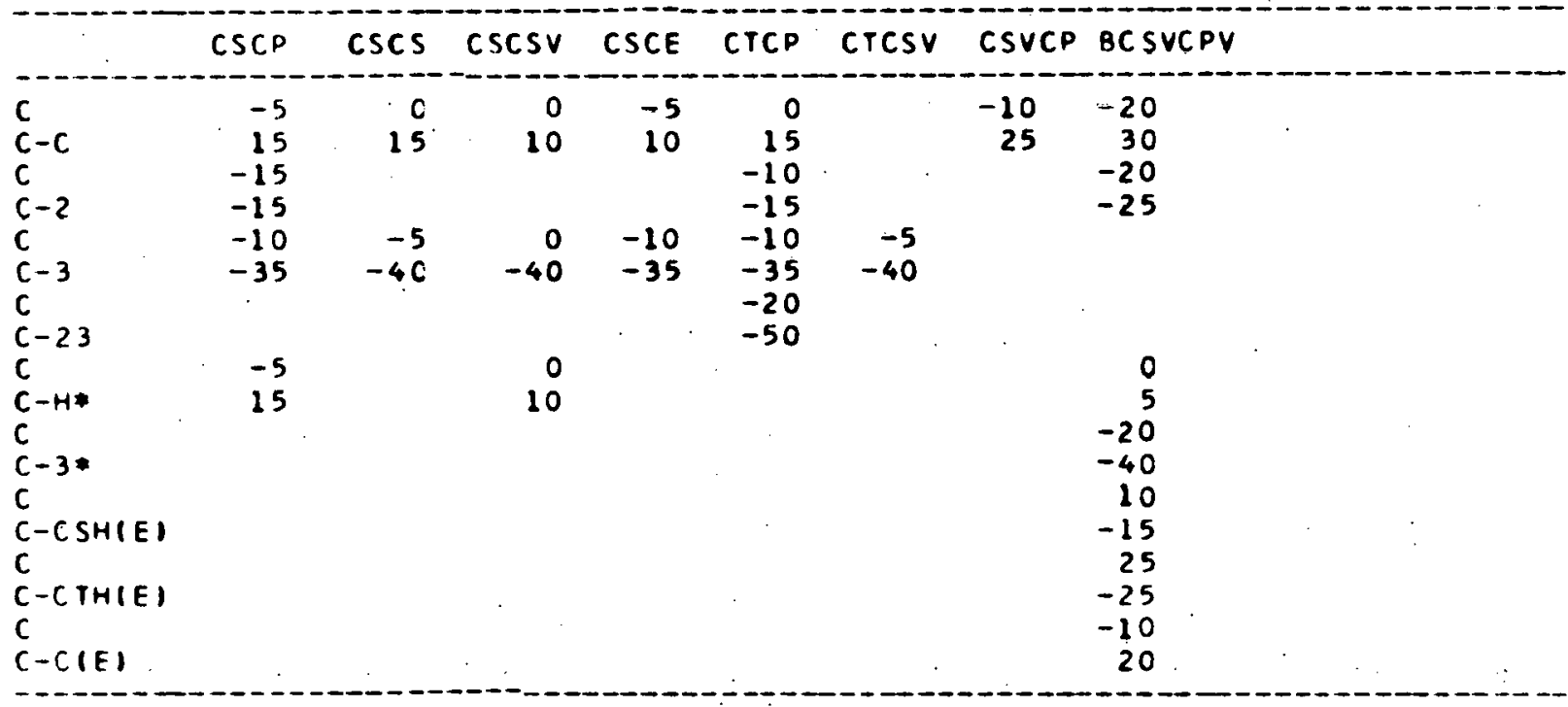


manifest is as observed for the $\mathrm{CH}$, and even the numbers are simi iar, i.e., the vicinal substituents affect the alkyl $\mathrm{CC}$ and $\mathrm{CH}$ in about the same ways.

\section{Primary Vinyl $\mathrm{CH}$ Bonds}

In mono- and disubstituted ethylenes, a primary $\mathrm{CH}$ bond can only have single bonds in vicinal positions. Thus there are three kinds of primary bond fragments depending on whether a carbon atom is in cis or in trans or in both vicinal positions. It is found that the bond fragment coefficients depend on bonds beyond the vicinal axes provided they are coplanar with the bond fragment. It is furthermore necessary to distinguish such coplanar bonds as regards their orientation with respect to the bond fragment. The possible cases are illustrated in Figures 3 and 4 and labelled by the abbreviations whose meanings are as follows: The letters $T, C, P$ and $L$ stand for 'trans', 'cis', 'plane' and 'line', respectively. In each three letter symbol, the first letter describes the orientation of the coplanar vicinal bond relative to the bond fragment, the second letter describes the orientation of the double bond relative to the coplanar third bond, and the last letter describes the orientation of the vicinal bond relative to the coplanar fourth bond. The symbol NFN means that there is no coplanar fourth bond. In this case the bond fragment turns out to be independent of the third bond as well, and only the orientation ( $T$ or $C$ ) of the vicinal bond is required. When no confusion is likely, we shall refer to these as simply NFN.

The bond fragment coefficients of the cis monosubstituted ethylenes differ substantially less from the ethylene coefficients than do those 
Figure 3. Bond skeletons for primary vinyl $\mathrm{CH}$ fragments cis to the substituent 


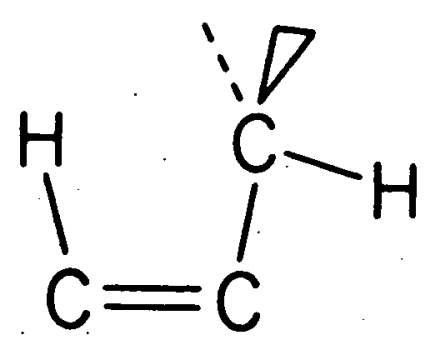

CNFN

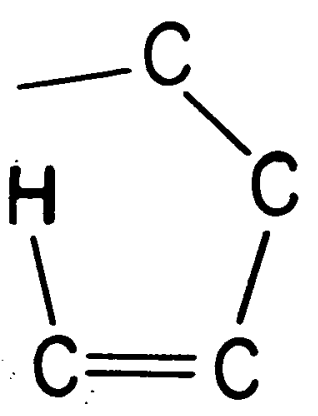

$\mathrm{CCC}$
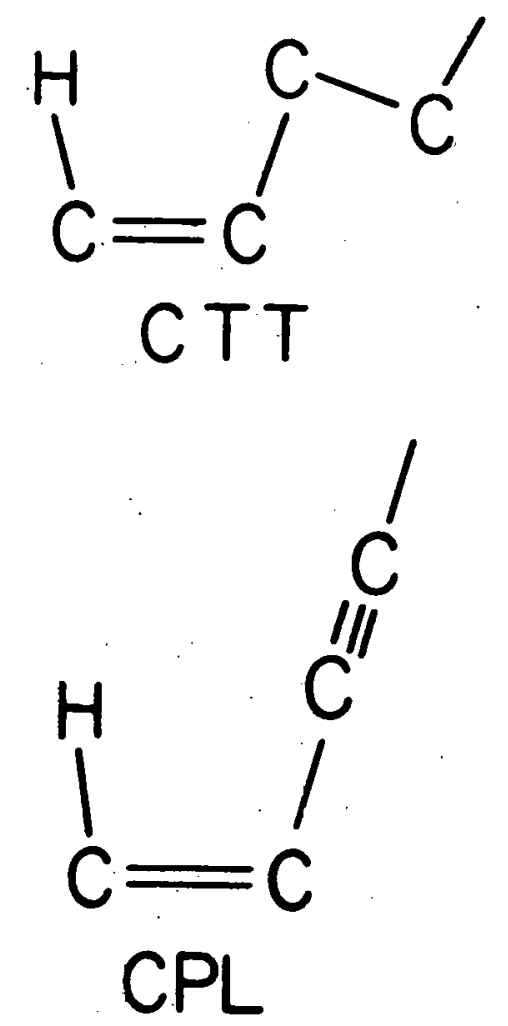
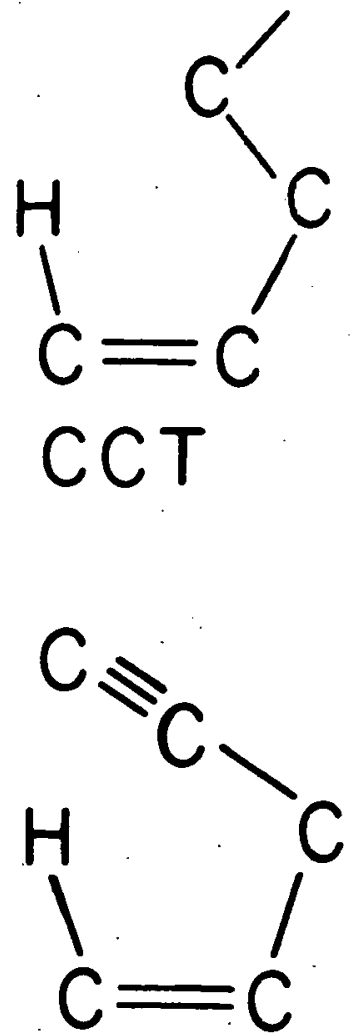

CCP 
Figure 4. Bond skeletons far primary vinyl $\mathrm{CH}$ fragments trans to the substituent 


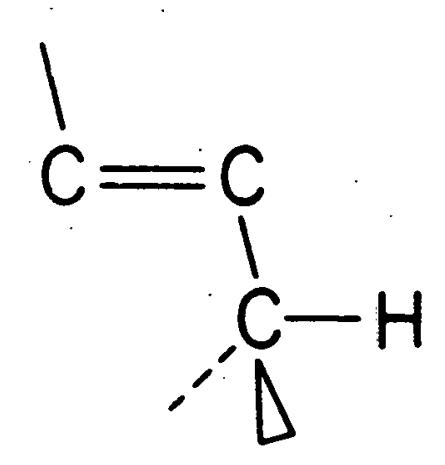

TNFN

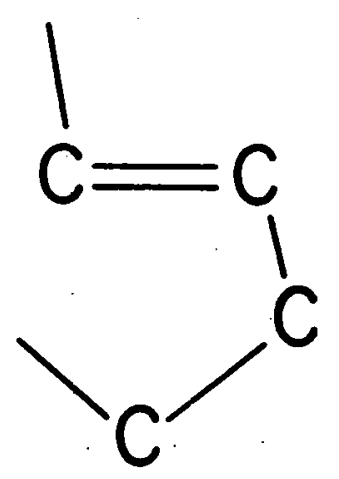

TCC

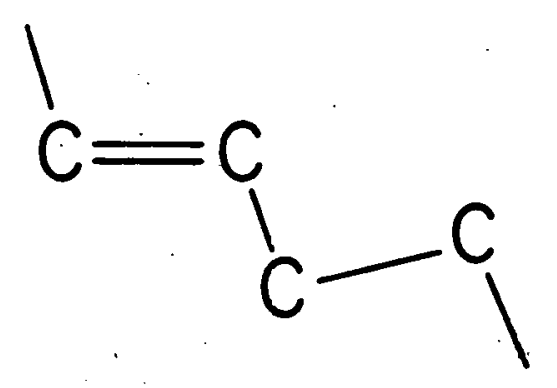

T T T

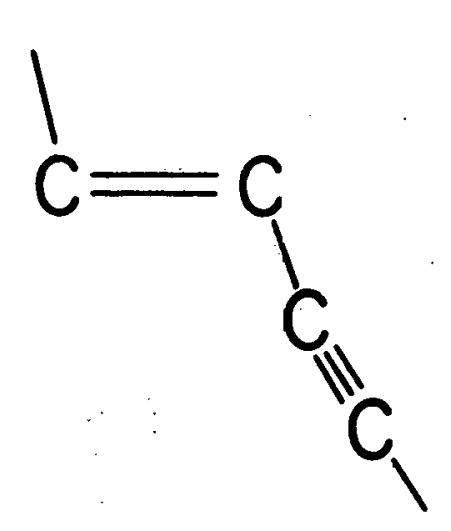

TPL

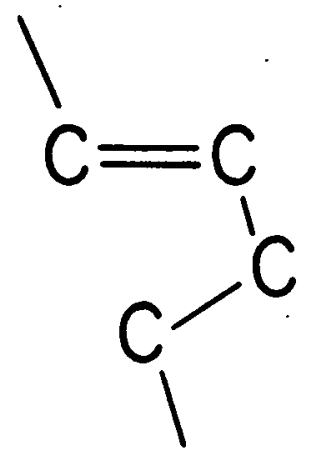

TCT

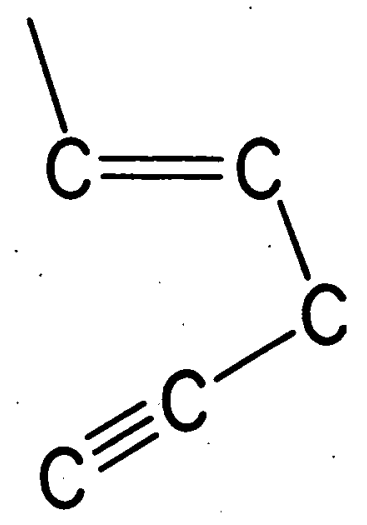

TCP 
of the trans monosubstituted ethylenes. The bond fragment coefficients in disubstituted ethylenes display the additive effects of both the cis and trans substituents. This means, in fact, that they behave very much like the trans monosubstituted ethylenes.

\section{Cis substituted ethylenes}

Ethylene and the $\mathrm{CH}$ fragments in cis monosubstituted ethylenes are listed in Table 47. The ones listed first are not much affected by the substituent, but the three listed last are appreciably changed from ethylene. Examination shows that these are configurations where the coplanar bonds in the molecule 'bend back' toward the bond fragment, so that the effect is partially steric (it is not wholly steric, because it occurs only when the bonds are coplanar). This is therefore a case where the geometric influence is as great as the influence of the type of the coplanar vicinal fragment that was found in the alkyl bonds. If this 'back bending' is absent, the Mulliken populations. on the Is are slightly greater than the $s p^{2}$.

\section{Trans substituted ethylenes}

For comparing with Table 47, the same bond categories are used in Table 48 to list the bond fragments for trans substituted ethylenes. However, in this case, the bond fragments are practically insensitive to the geometries of the substituents, except for a small change when the double bond is conjugate to a triple bond. The Mulliken populations again favor the is over the $S p^{2}$, and by amounts slightly larger than the cis cases. 
TABLE 47. BONO ANO GEMINAL FRAGMENTS IN ETHYLENE AND PRIMARY VINYL CH LMC'S CIS TO THE SUBSTITUEN

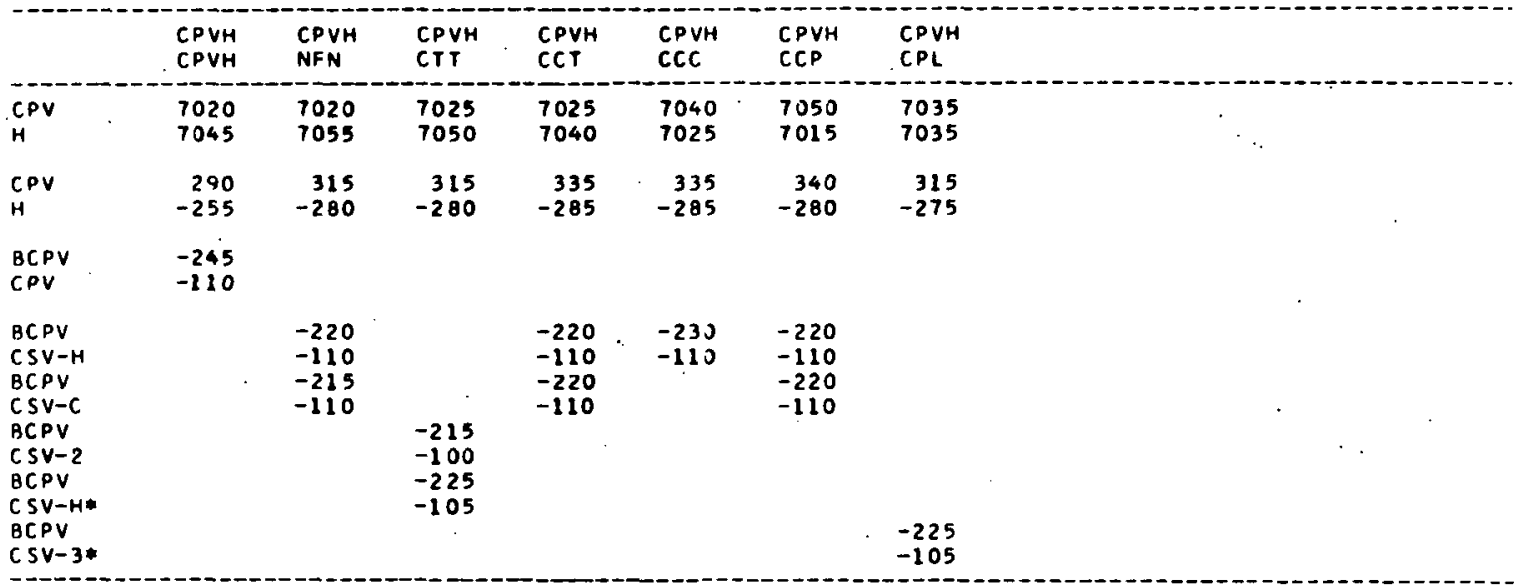


TABLE 48. ROND AND CEMINAL FRAGMENTS IN ETHYLENE ANO PRIMARY VINYL CH LMO'S TRANS TD THE SUBSTITUENT

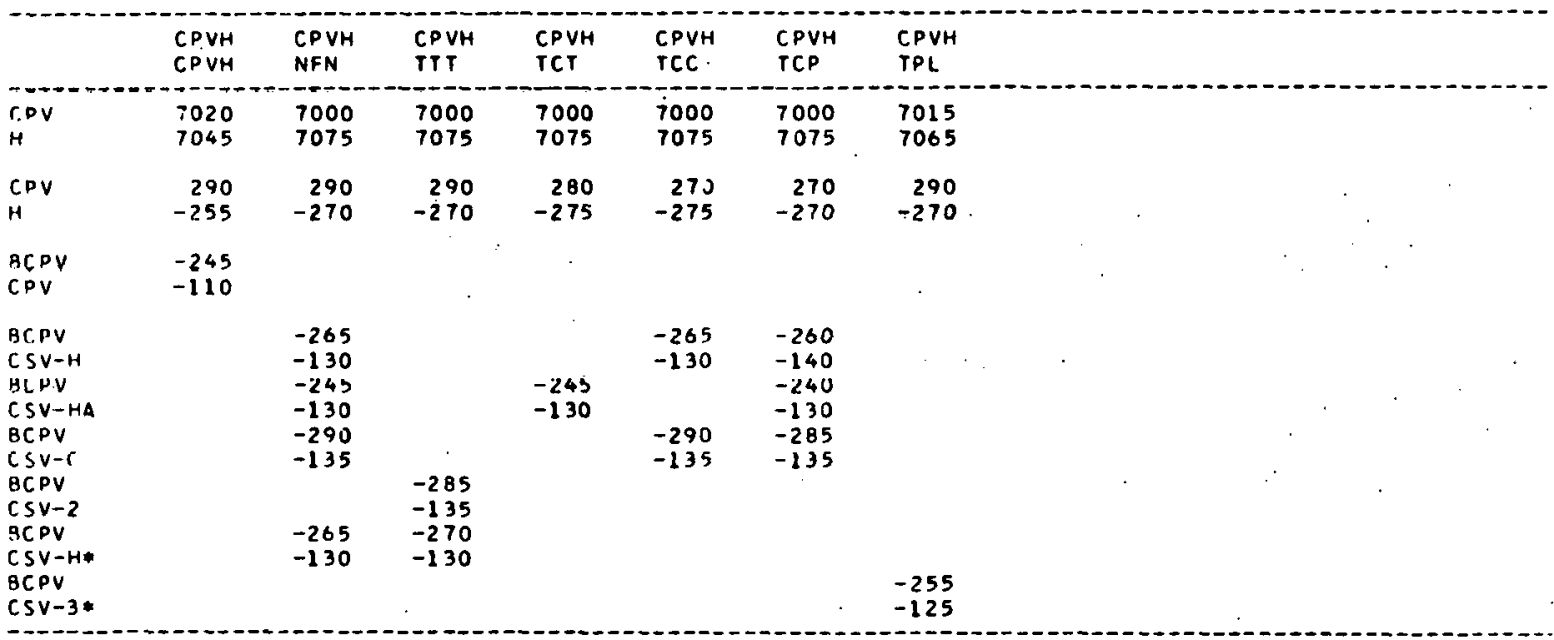




\section{Disubstituted ethylenes}

Only one substituent is labelled for the disubstituted ethylenes in Table 49: the other is necessarily the corresponding TNFN or CNFN. For example, the CTT in the second column refers to the cis position, the trans being TNFN (written as NFN in the table). The character of the trans substituent is the major determinant, and the bond fragment coefficients differ little from those of the trans monosubstituents (Table 48 ). If the trans substituent is TNFN, then the various cis substituents run parallel to those of the cis monosubstituted cases (Table 47).

\section{Secondary Vinyl $\mathrm{CH}$ Bonds}

The bond fragments of secondary vinyl $\mathrm{CH}$ bonds (Table 50) resemble more closely the alkyl $\mathrm{CH}$ bonds than the primary vinyl $\mathrm{CH}$ bonds, which implies that the geminal single bond has a greater influence than the geminal double bond. Similarly, we find that the vicinal substituents beyond the geminal single bond are more important than those beyond the geminal double bond. For example, there is little change in the bond fragment when a CNFN substituent is placed at the end of the double bond (see CSVH-CNFN column). Therefore the other columns deal with bond fragments having various possible vicinal substitutions beyond the single bond. The differences in bond fragment coefficients corresponding to these substitutions were also included in the previous Table 40 . The agreement with the alkyl $\mathrm{CH}$ bonds is perhaps closer than expected.

The column CSVH-CCC corresponds to the case that cis single bonds are in vicinal, third and fourth positions beyond the single bond. This 


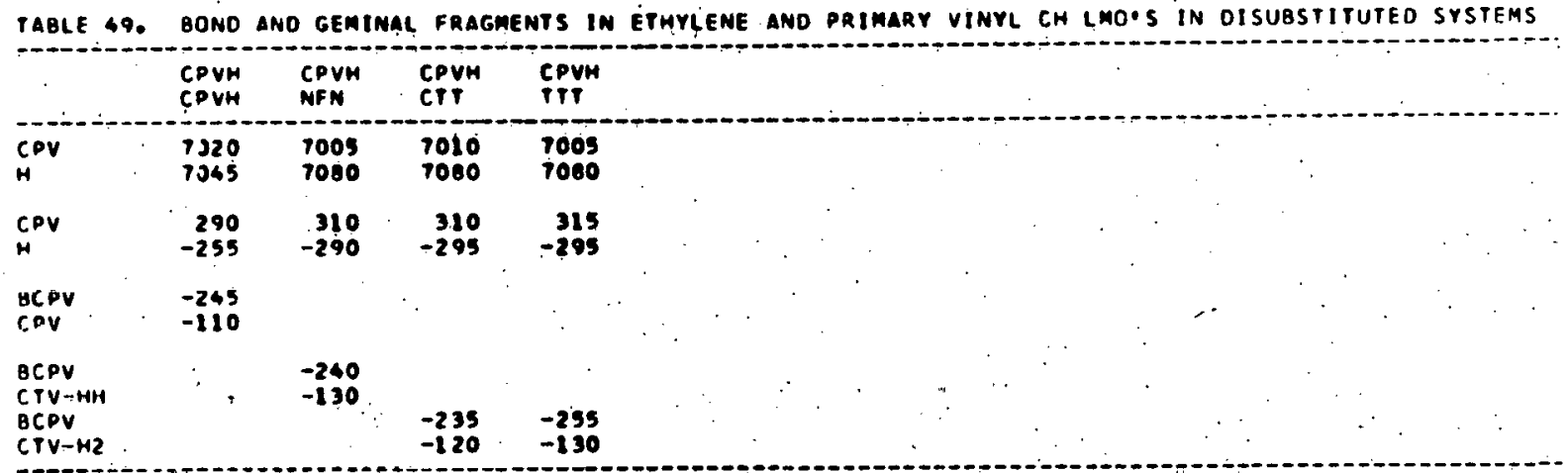


TABLE 50. BONO AND GEMINAL FRAGMENTS IN SECONDARY VINYL CH LMO'S

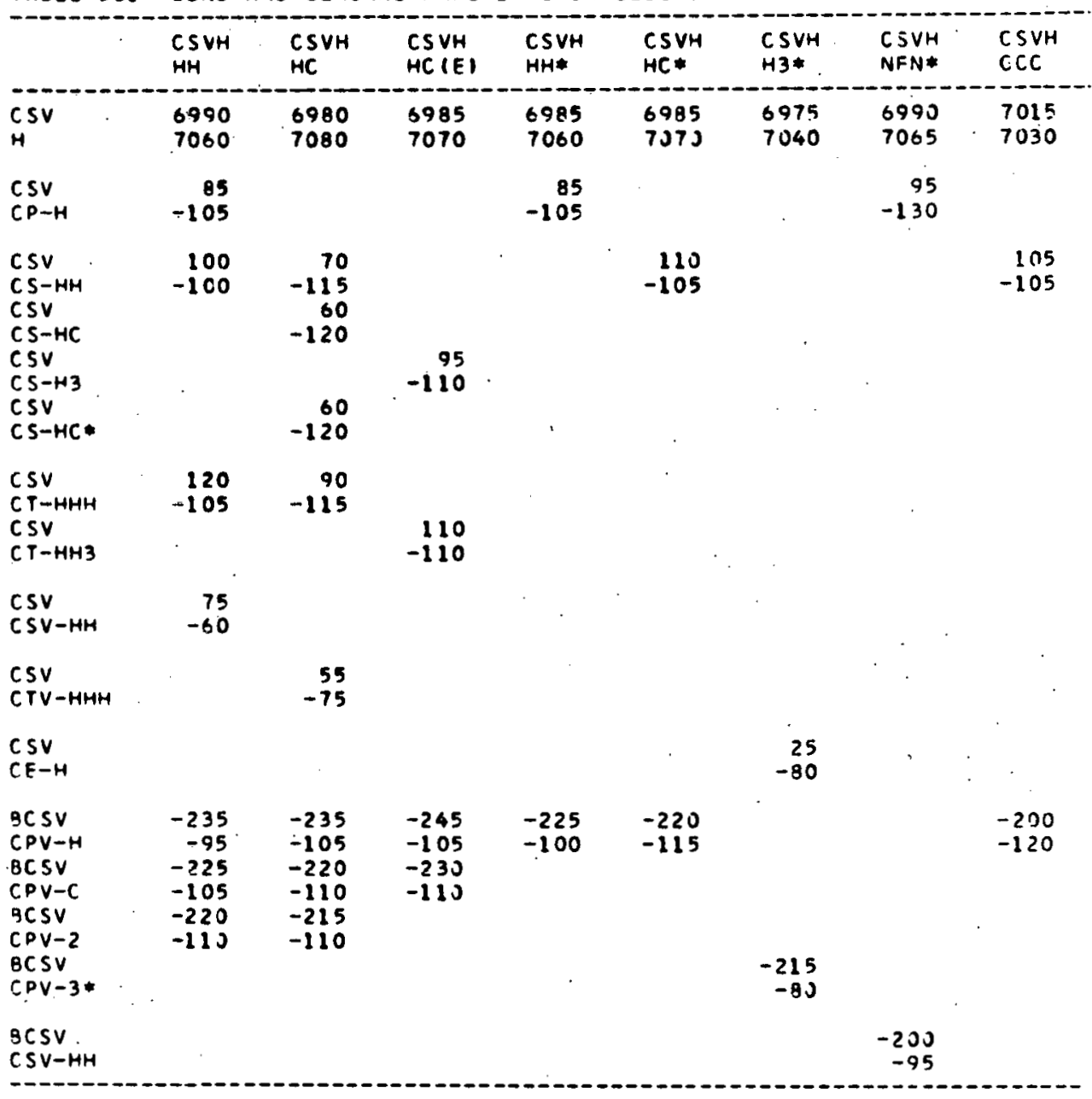


is an example for a partial steric effect occurring across single bonds. It may be noted that this substitution of the CCC tail beyond the geminal single bond affects the bond fragment in almost the same way as the substitution of the cccitail beyond the geminal double bond, shown previously in Table 47. The coplanar orientation seems to be more important than the geminal bond.

\section{Vinyl CC Single Bonds}

Many of the secondary vinyl CC single bonds were discussed in the previous section on alkyl CC bonds. This applies to all cases listed in Table 51, with the exception of the bonds having a TNFN vicinal substituent beyond the geminal double bond, given in the column CSVCP-NFN. By comparing with Table 48, one sees that this substitution has the same effect on the (CC) bond fragments CSVCP as it had in the (CH) bond fragments $\mathrm{CPVH}$.

Table 52 exhibits secondary and tertiary vinyl CC single bond fragments not including alkyl carbons. The equality of the coefficients in the CSVCTV fragment justify the polarity ranking given in Equation 53, but it should be pointed out that there is a difference between the coefficients in the CSVCSV and CSVCTV bond fragments. In these, and also in the CVCE, one expects geometric and type effects similar to those of the CSVH.

\section{Double Bonds}

A double bond contiains the bond fragments of two LMO's which essentially describe the bond. These bond fragments have 'banana character', i.e., they are bent above and below the nodal plane of the participating pi orbitals. 
TABLE SL. BONO ANO GEMINAL FRAGMENTS IN VINYL CC(ALKYL) LMOOS

\begin{tabular}{|c|c|c|c|c|c|c|c|c|c|}
\hline & ${ }_{H}^{C S V C P}$ & $\begin{array}{l}\text { CSVCP } \\
\text { NFN }\end{array}$ & $\begin{array}{l}\text { CSVES } \\
\text { HH }\end{array}$ & $\begin{array}{l}\text { csves } \\
\mathrm{HC}\end{array}$ & $\begin{array}{l}\text { Csves } \\
\mathrm{H3}\end{array}$ & $\begin{array}{l}\text { Csves } \\
\text { HH* }\end{array}$ & $\begin{array}{l}\text { CSVCT } \\
\text { HMH }\end{array}$ & $\begin{array}{l}\text { C SVCT } \\
\text { HH3 }\end{array}$ & $\begin{array}{l}\text { CTVCP } \\
\text { HH }\end{array}$ \\
\hline $\begin{array}{l}c V \\
c\end{array}$ & $\begin{array}{l}7030 \\
7040\end{array}$ & $\begin{array}{l}7020 \\
7065\end{array}$ & $\begin{array}{l}7015 \\
7015\end{array}$ & $\begin{array}{l}7025 \\
7015\end{array}$ & $\begin{array}{l}6975 \\
7015\end{array}$ & $\begin{array}{l}7025 \\
7010\end{array}$ & $\begin{array}{l}7000 \\
6995\end{array}$ & $\begin{array}{l}6760 \\
5990\end{array}$ & $\begin{array}{l}7010 \\
7030\end{array}$ \\
\hline $\begin{array}{l}\text { CSV } \\
H-H H\end{array}$ & $\begin{array}{r}275 \\
-175\end{array}$ & $\begin{array}{r}280 \\
-190\end{array}$ & $\begin{array}{r}265 \\
-185\end{array}$ & & 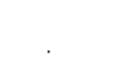 & & $\begin{array}{r}250 \\
-190\end{array}$ & & \\
\hline $\begin{array}{l}\text { CSV } \\
H \rightarrow H C \\
\text { CSV } \\
H-H H *\end{array}$ & $\begin{array}{r}280 \\
-175\end{array}$ & & $\begin{array}{r}315 \\
-190\end{array}$ & $\begin{array}{r}330 \\
-190\end{array}$ & $\begin{array}{r}305 \\
-190\end{array}$ & $\begin{array}{r}330 \\
-190\end{array}$ & $\begin{array}{r}305 \\
-195\end{array}$ & $\begin{array}{r}295 \\
-195\end{array}$ & \\
\hline $\begin{array}{l}C S V \\
H-H C\end{array}$ & & & $\begin{array}{r}260 \\
-185\end{array}$ & & & & & & \\
\hline $\begin{array}{l}C T V \\
C P-M H\end{array}$ & & & &. & & & : & & $\begin{array}{l}135 \\
-65\end{array}$ \\
\hline $\begin{array}{l}\text { CTV } \\
\text { CSV-HHH }\end{array}$ & . & & & & & & & & $\begin{array}{r}75 \\
-15\end{array}$ \\
\hline $\begin{array}{l}B C V \\
C P V-H \\
B C V \\
C P V-H A \\
B C V\end{array}$ & $\begin{array}{r}-220 \\
-55\end{array}$ & & $\begin{array}{r}-235 \\
-65 \\
-275 \\
-50\end{array}$ & $\begin{array}{r}-235 \\
-65\end{array}$ & $\begin{array}{r}-240 \\
-65\end{array}$ & $\begin{array}{r}-240 \\
-65\end{array}$ & $\begin{array}{r}-290 \\
-55\end{array}$ & $\begin{array}{r}-300 \\
-55\end{array}$ & $\begin{array}{r}-200 \\
-45\end{array}$ \\
\hline & & . & & & & V & & & $\begin{array}{r}-i 9 J \\
-55\end{array}$ \\
\hline $\begin{array}{l}B C V \\
C P V-C A \\
B C V \\
C P V-H\end{array}$ & . & v & $\begin{array}{r}-145 \\
-65\end{array}$ & & & & $\begin{array}{r}-155 \\
-75\end{array}$ & $\begin{array}{r}-165 \\
-75\end{array}$ & \\
\hline $\begin{array}{l}B C V \\
\text { CPV-H* }\end{array}$ & $\begin{array}{r}-225 \\
-55\end{array}$ & & $\begin{array}{r}-195 \\
-60\end{array}$ & & & & & . & \\
\hline $\begin{array}{l}\text { BCSS } \\
\text { CSV-HH }\end{array}$ & & $\begin{array}{r}-240 \\
-75\end{array}$ & & & . & & . & & \\
\hline
\end{tabular}


IABLE 52. BOND AND GEMINAL FRAGMENTS IN VINYL CCIVINYL OR ETHYNYLI LHO.S

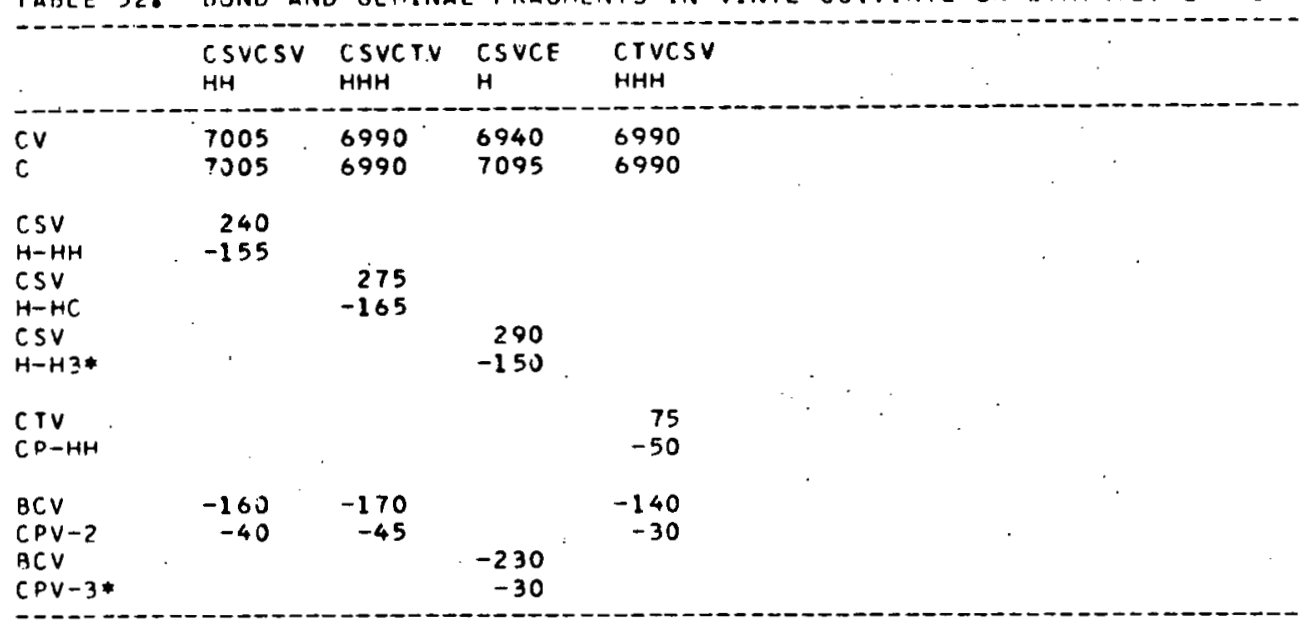


TABLE 53. BOND AND GEMINAL FRAGMENTS ON PRIMARY CARBONS IN DOUBLE Banana lMO'S

\begin{tabular}{|c|c|c|c|c|c|c|c|c|c|}
\hline & BCPVCPV & $\begin{array}{l}\text { BCPVCSV } \\
\mathbf{H}\end{array}$ & $\begin{array}{l}\text { BCPVCSV } \\
\text { C }\end{array}$ & $\begin{array}{l}\text { gCPVCSV } \\
2\end{array}$ & $\begin{array}{l}\text { BCPVCSV } \\
\text { CSH(E) }\end{array}$ & $\begin{array}{l}\text { BCPVCSV } \\
\text { CTHIEI }\end{array}$ & $\begin{array}{l}\text { BCPVCSV } \\
\text { CTCIEI }\end{array}$ & $\begin{array}{l}\text { BCPVCSV } \\
\mathrm{H}^{*}\end{array}$ & $\begin{array}{l}\text { SCPVCSV } \\
2 *\end{array}$ \\
\hline $\begin{array}{l}B C P V \\
C V\end{array}$ & $\begin{array}{l}7055 \\
7055\end{array}$ & $\begin{array}{l}7060 \\
7010\end{array}$ & $\begin{array}{l}7090 \\
6990\end{array}$ & $\begin{array}{l}7035 \\
6990\end{array}$ & $\begin{array}{l}7045 \\
7020\end{array}$ & $\begin{array}{l}7035 \\
7035\end{array}$ & $\begin{array}{l}7080 \\
7000\end{array}$ & $\begin{array}{l}7065 \\
7010\end{array}$ & $\begin{array}{l}7020 \\
6990\end{array}$ \\
\hline $\begin{array}{l}C P V \\
H-H \\
C P V \\
H-C\end{array}$ & $\begin{array}{r}340 \\
15\end{array}$ & $\begin{array}{r}320 \\
10 \\
375 \\
0\end{array}$ & $\begin{array}{r}320 \\
10 \\
400 \\
0\end{array}$ & $\begin{array}{r}320 \\
10 \\
430 \\
0\end{array}$ & $\begin{array}{r}320 \\
10 \\
385 \\
0\end{array}$ & $\begin{array}{r}320 \\
10 \\
365 \\
j\end{array}$ & $\begin{array}{r}320 \\
10 \\
400 \\
0\end{array}$ & $\begin{array}{r}320 \\
15 \\
375 \\
0\end{array}$ & $\begin{array}{r}320 \\
10 \\
380 \\
0\end{array}$ \\
\hline $\begin{array}{l}\text { BCPV } \\
\text { CPV }\end{array}$ & $\begin{array}{l}-15 \\
-15\end{array}$ & & · & & . & & & & \\
\hline $\begin{array}{l}\text { BCPV } \\
\text { CSV-H } \\
\text { BCPV } \\
\text { CSV-C } \\
\text { BCPV } \\
\text { CSV-2 }\end{array}$ & & $\begin{array}{r}-60 \\
50 \\
-85 \\
50\end{array}$ & $\begin{array}{r}-80 \\
80 \\
-95 \\
80\end{array}$ & $\begin{array}{r}-10 \\
50\end{array}$ & $\begin{array}{r}-45 \\
40\end{array}$ & $\begin{array}{r}-75 \\
35\end{array}$ & $\begin{array}{r}-60 \\
70\end{array}$ & $\begin{array}{r}-50 \\
40\end{array}$ & \\
\hline $\begin{array}{l}\text { BCPV } \\
\text { CSV } 3 *\end{array}$ & & & & & & & & & $\begin{array}{r}-20 \\
40\end{array}$ \\
\hline
\end{tabular}


Tables 53, 54, and 55 give the coefficients of the constituent hybrids for the cases that the bonded atoms are primary-primary, primary-secondary, primary-tertiary and secondary-secondary carbons.

It is seen that the coefficients do not differ greatly from those found for the single $C C$ bonds. This is so even in the cases of conjugated bonds, which may seem surprising in view of the greater delocalization. The banana bond coefficients are, however, more sensitive to vicinal substituents than are the single bonds. These substituent effects were also included in Table 46. But on the other hand, these substituent effects are quite independent of whether the carbon atoms forming the double bond are secondary or tertiàry. This latter result suggests that our observations are likely to hold generally, for double bonds although our sampling is limited.

\section{Ethynyl $\mathrm{CH}$ Bonds}

The ethynyl $\mathrm{CH}$ bond fragments (see Table 56) exhibit the strongest polarity encountered in any bond fragments examined. in this investigation. Moreover, they are the only $\mathrm{CH}$ bonds in which the electron(Mulliken) population on the carbon is greater than that on the hydrogen, in agreement with chemical ideas as regards the 'acidity' of acetylene.

Another characteristic is that the coefficients in the bond fragment are practically insensitive to the substituents on the other side of the triple bond, except if another triple bond is added conjugate with the first. Therefore, Table 56 lists only two classes of ethynyl $\mathrm{CH}$ bonds, those with and those without a second conjugated triple bond. Note that 
TARLE 53. BOND ANO GEMINAL FRAGMENTS ON PRIMARY CARBONS IN DOUBLE BANANA LMO'S

\begin{tabular}{|c|c|c|c|c|c|c|c|c|c|}
\hline & RCPVCDV & $\begin{array}{l}\text { BCPVCSV } \\
M\end{array}$ & $\begin{array}{l}\text { BCPVCSV } \\
\text { C }\end{array}$ & $\begin{array}{l}\text { BCPVCSV } \\
2\end{array}$ & $\begin{array}{l}\text { BCPVCSV } \\
\text { CSHIEI }\end{array}$ & $\begin{array}{l}\text { BCPVCSV } \\
\text { CTHIEI. }\end{array}$ & $\begin{array}{l}\text { BCPUCSV } \\
\text { CTCIEI }\end{array}$ & $\begin{array}{l}\text { BCPVCSV } \\
\text { * }\end{array}$ & $\begin{array}{l}\text { SCPVCSV } \\
? *\end{array}$ \\
\hline $\begin{array}{l}P C P V . \\
C V\end{array}$ & $\begin{array}{l}7.355 \\
705.5\end{array}$ & $\begin{array}{l}7060 \\
7010\end{array}$ & $\begin{array}{l}7090 \\
6990\end{array}$ & $\begin{array}{l}7035 \\
6990\end{array}$ & $\begin{array}{l}7045 \\
7020\end{array}$ & $\begin{array}{l}7035 \\
7035\end{array}$ & $\begin{array}{l}7080 \\
7000\end{array}$ & $\begin{array}{l}7065 \\
7010\end{array}$ & $\begin{array}{l}702.0 \\
6990\end{array}$ \\
\hline $\begin{array}{l}\text { CPV } \\
H-H \\
\text { COV } \\
H-C\end{array}$ & $\begin{array}{r}340 \\
15\end{array}$ & $\begin{array}{r}320 \\
10 \\
375 \\
0\end{array}$ & $\begin{array}{r}320 \\
10 \\
400 \\
0\end{array}$ & $\begin{array}{r}320 \\
10 \\
430 \\
0\end{array}$ & $\begin{array}{r}320 \\
10 \\
385 \\
0\end{array}$ & $\begin{array}{r}320 \\
13 \\
355 \\
3\end{array}$ & $\begin{array}{r}320 \\
10 \\
40 J \\
0\end{array}$ & $\begin{array}{r}320 \\
15 \\
375 \\
0\end{array}$ & $\begin{array}{r}320 \\
10 \\
380 \\
0\end{array}$ \\
\hline $\begin{array}{l}\text { PCPV } \\
\text { CPV }\end{array}$ & $\begin{array}{l}-15 \\
-15\end{array}$ & & & & & & & & \\
\hline $\begin{array}{l}\text { SCPV } \\
\text { CSV-H } \\
\text { BCPV } \\
\text { CSV-C. } \\
\text { BCPV } \\
\text { CSV-2 } \\
\text { SCPV } \\
\text { CSV-3* }\end{array}$ & $\cdot$ & $\begin{array}{r}-60 \\
50 \\
-85 \\
50\end{array}$ & $\begin{array}{r}-80 \\
80 \\
-95 \\
80\end{array}$ & $\begin{array}{r}-10 \\
50\end{array}$ & $\begin{array}{r}-45 \\
40\end{array}$ & $\begin{array}{r}-75 \\
35\end{array}$ & $\begin{array}{r}-60 \\
70\end{array}$ & $\begin{array}{r}-50 \\
40\end{array}$ & $\begin{array}{r}-2 J \\
4.3\end{array}$ \\
\hline
\end{tabular}


TABLE 54. BOND ANO GEMINAL FRAGMENTS ON PRIMARY AND TERTIARY CARBONS IN DJUBLE BANANA LMO'S

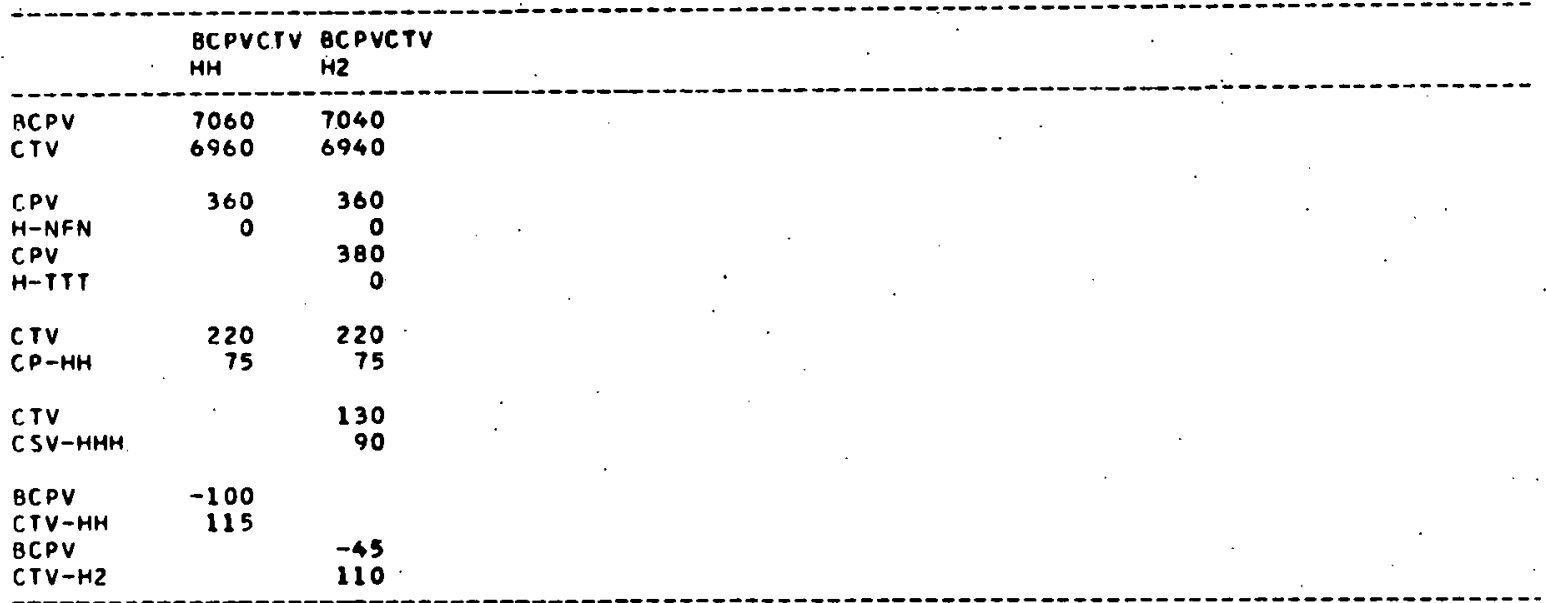


111

TARLE 55. BOND ANO GEMINAL FRAGMENTS ON SECONDARY CARBONS IN BANANA LMO'S

\begin{tabular}{|c|c|c|c|c|c|c|c|c|c|}
\hline & $\begin{array}{l}\text { BCSVCPV } \\
\mathrm{H}\end{array}$ & $\begin{array}{l}\text { BCSVCPV } \\
\mathrm{C}\end{array}$ & $\begin{array}{l}\text { BC SVCPV } \\
2\end{array}$ & $\begin{array}{l}\text { ACSVCPV } \\
\text { CSHIEI }\end{array}$ & $\begin{array}{l}\text { BCSVCPV } \\
\text { CTHIEI }\end{array}$ & $\begin{array}{l}\text { BCSVCPV } \\
\text { CTCIE) }\end{array}$ & $\begin{array}{l}\text { BCSVCPV } \\
\mathrm{H} \text {. }\end{array}$ & $\begin{array}{l}\text { BC SVCPV } \\
3 *\end{array}$ & $\begin{array}{l}\text { BCSVCSV } \\
\text { HH }\end{array}$ \\
\hline $\begin{array}{l}\text { ACSV } \\
C V\end{array}$ & $\begin{array}{l}7010 \\
7060\end{array}$ & $\begin{array}{l}6990 \\
7090\end{array}$ & $\begin{array}{l}6990 \\
7035\end{array}$ & $\begin{array}{l}7020 \\
7045\end{array}$ & $\begin{array}{l}7035 \\
7035\end{array}$ & $\begin{array}{l}7000 \\
7080\end{array}$ & $\begin{array}{l}7010 \\
7065\end{array}$ & $\begin{array}{l}6990 \\
7020\end{array}$ & $\begin{array}{l}7015 \\
7010\end{array}$ \\
\hline $\begin{array}{l}\text { CSV } \\
\text { H-HH }\end{array}$ & $\begin{array}{r}375 \\
0\end{array}$ & $\begin{array}{r}370 \\
-10\end{array}$ & $\begin{array}{r}370 \\
-10\end{array}$ & & & & $\begin{array}{l}369 \\
-10\end{array}$ & & $\begin{array}{r}350 \\
-10\end{array}$ \\
\hline $\begin{array}{l}\text { CSV } \\
\text { H-HC }\end{array}$ & $\begin{array}{r}380 \\
-10\end{array}$ & $\begin{array}{l}380 \\
-20\end{array}$ & $\begin{array}{r}375 \\
-15\end{array}$ & $\begin{array}{r}390 \\
-10\end{array}$ & $\begin{array}{r}390 \\
-10\end{array}$ & $\begin{array}{l}380 \\
-20\end{array}$ & & & . \\
\hline $\begin{array}{l}\mathrm{CSV} \\
\mathrm{H}-\mathrm{H3}\end{array}$ & & . &. & & & & & $\begin{array}{r}360 \\
10\end{array}$ & \\
\hline $\begin{array}{l}\text { CSV. } \\
\text { CP-H }\end{array}$ & $\begin{array}{r}200 \\
85\end{array}$ & & . & & & & $\begin{array}{r}200 \\
85\end{array}$ & & \\
\hline $\begin{array}{l}\text { CSV } \\
\text { CP } P-C\end{array}$ & & & & & & & & & $\begin{array}{r}235 \\
80\end{array}$ \\
\hline $\begin{array}{l}\text { CSV } \\
\text { CS-HH }\end{array}$ & $\begin{array}{r}225 \\
90\end{array}$ & $\begin{array}{r}170 \\
60\end{array}$ & & & - & & $\begin{array}{r}190 \\
80\end{array}$ & & \\
\hline $\begin{array}{l}\text { CSV } \\
\text { CS }-H C\end{array}$ & $\begin{array}{r}220 \\
80\end{array}$ & & & & & & & & \\
\hline $\begin{array}{l}\mathrm{CSV} \\
\mathrm{CS}-\mathrm{H}_{3}\end{array}$ & & & & $\begin{array}{r}215 \\
80\end{array}$ & & & & & \\
\hline $\begin{array}{l}\text { CSV } \\
\text { CT-HHH }\end{array}$ & $\begin{array}{l}250 \\
200\end{array}$ & $\begin{array}{r}185 \\
75\end{array}$ & & & & & & & \\
\hline $\begin{array}{l}\mathrm{CSV} \\
\mathrm{CT}-\mathrm{HH}_{3}\end{array}$ & & & . & & $\begin{array}{r}250 \\
95\end{array}$ & $\begin{array}{r}180 \\
60\end{array}$ & & & \\
\hline $\begin{array}{l}\text { CSV } \\
\text { C SV-HH }\end{array}$ & & & $\begin{array}{l}110 \\
100\end{array}$ & & & $\cdot$ & & & \\
\hline $\begin{array}{l}\text { CSV } \\
\text { CTV-HMH }\end{array}$ & . & & $\begin{array}{l}130 \\
100\end{array}$ & & & & & & \\
\hline $\begin{array}{l}\text { CSV } \\
\text { CE }-H\end{array}$ & & & & & & & & $\begin{array}{l}160 \\
120\end{array}$ & \\
\hline $\begin{array}{l}B C S V \\
C V-H\end{array}$ & $\begin{array}{r}50 \\
-60\end{array}$ & $\begin{array}{r}00 \\
-80\end{array}$ & & $\begin{array}{r}40 \\
-45\end{array}$ & & $\begin{array}{r}70 \\
-60\end{array}$ & $\begin{array}{r}60 \\
-50\end{array}$ & & $\begin{array}{l}10 \\
10\end{array}$ \\
\hline $\begin{array}{l}\text { BC SV } \\
\text { CPV -C }\end{array}$ & $\begin{array}{r}50 \\
-85\end{array}$ & $\begin{array}{r}80 \\
-95\end{array}$ & & & $\begin{array}{r}35 \\
-75\end{array}$ & & & & \\
\hline $\begin{array}{l}B C S V \\
C P V-2\end{array}$ & & & $\begin{array}{r}50 \\
-10\end{array}$ & & & & & & \\
\hline $\begin{array}{l}B C S V \\
C P V-3 *\end{array}$ & & & & & $\therefore$ & & & $\begin{array}{r}40 \\
-20\end{array}$ & \\
\hline
\end{tabular}


TABLE 56. BONO ANO GEMINAL FRAGMENTS ON ETHYNYL CARBONS IN CH ANO CC LMO'S

\begin{tabular}{|c|c|c|c|c|c|c|c|c|c|}
\hline & CEH & $\begin{array}{l}\text { CEH } \\
\text { (E) }\end{array}$ & CECP & ${ }_{H}^{\text {CECS }}$ & $\begin{array}{l}\text { CECS } \\
\text { C }\end{array}$ & $\begin{array}{l}\text { CECS } \\
3\end{array}$ & $\begin{array}{l}\text { CECT } \\
H H\end{array}$ & ${ }_{H}^{C E C S V}$ & CECE \\
\hline $\begin{array}{l}C E \\
H, C\end{array}$ & $\begin{array}{l}7230 \\
6875\end{array}$ & $\begin{array}{l}7245 \\
6860\end{array}$ & $\begin{array}{l}7120 \\
6985\end{array}$ & $\begin{array}{l}7110 \\
6950\end{array}$ & $\begin{array}{l}7120 \\
6955\end{array}$ & $\begin{array}{l}7075 \\
6950\end{array}$ & $\begin{array}{l}7090 \\
6935\end{array}$ & $\begin{array}{l}7095 \\
6940\end{array}$ & $\begin{array}{l}7.040 \\
7040\end{array}$ \\
\hline $\begin{array}{l}\text { BCPE } \\
\text { CPE }\end{array}$ & $\begin{array}{r}5 \\
-335\end{array}$ & & & & & & & . & \\
\hline $\begin{array}{l}\text { RCE } \\
C E-H \\
B C E \\
C E-C\end{array}$ & $\begin{array}{r}10 \\
-335 \\
-10 \\
-345\end{array}$ & & & $\begin{array}{r}-40 \\
-290 \\
95 \\
-290\end{array}$ & $\begin{array}{r}-40 \\
-290 \\
90 \\
-290\end{array}$ & $\begin{array}{r}-40 \\
-290 \\
80 \\
-290\end{array}$ & $\begin{array}{r}-75 \\
-290 \\
50 \\
-290\end{array}$ & & \\
\hline $\begin{array}{l}\text { BCE } \\
C E-3 \\
B C E \\
C E-H *\end{array}$ & $\begin{array}{r}0 \\
-335\end{array}$ & $\begin{array}{r}-5 \\
-335\end{array}$ & $\begin{array}{r}0 \\
-285\end{array}$ & & & & & & $\begin{array}{r}-5 \\
-250\end{array}$ \\
\hline $\begin{array}{l}\text { RCE } \\
\text { CE-VH* }\end{array}$ & $\begin{array}{r}-30 \\
-345\end{array}$ & & & & & & & $\begin{array}{r}140 \\
-285\end{array}$ & \\
\hline $\begin{array}{l}\text { BCE } \\
\text { CE-2* }\end{array}$ & $\begin{array}{r}15 \\
-330\end{array}$ & & & & & & & $\begin{array}{r}-70 \\
-285\end{array}$ & \\
\hline $\begin{array}{l}\text { BCSE } \\
\text { CSE-HH }\end{array}$ & & & $\begin{array}{r}-10 \\
-295\end{array}$ & & & & & & \\
\hline
\end{tabular}


a double bond in conjugation with the triple bond has no noticeable effect. Note also that such conjugation effects were found for the CPVH bonds (Tables 47,48$)$.

\section{Ethynyl CC Single Bonds}

Bond fragments for the $C C$ single bonds next to a triple bond are also listed in Table 56. They behave fairly similar to the analogous $\mathrm{CH}$ bonds. The bond fragments are polarized toward the ethynyl carbon, however considerably less so than the $\mathrm{CH}$ bonds. The fragments are very insensitive as regards vicinal substitution beyond the triple bond, with the exception of a second conjugate triple bond.

On the other hand, these single bonds can be part of a larger alkyl chain 'this side' of the triple bond. The vicinal substituents of such chains all change the bond fragment coefficients by the increments discussed in Table 46.

If the ethynyl CC bond has a double bond in a second geminal position, the effects discussed for the vinyl CC bonds are expected to add. A single bond fragment between two triple bonds has coefficients more nearly iike alkyl and vinyl single bond fragments and is expected to be extremely insensitive to any vicinal substitution.

\section{Triple Bonds}

A triple bond contains the bond fragments of three LMO's which essentially describe the bond. Like the bond fragments of the double bonds, these too have banana character and their coefficients (Tables 57,58 ) are close to those of the CC single bond fragments. Another similarity among 
114

TABI.E 57. BJND AND GEMINAL FRAGMENTS ON PRIMARY ETHYNYL CARBONS IN BANANA LMO'S

\begin{tabular}{|c|c|c|c|c|c|c|c|c|c|}
\hline & RCPECPE & $\begin{array}{l}\text { BCPECSE } \\
\text { CPH* }\end{array}$ & $\begin{array}{l}\text { BCPECSE } \\
\text { CSH }\end{array}$ & $\begin{array}{l}\text { BCPECSE } \\
\text { CTH }\end{array}$ & $\begin{array}{l}\text { BCPECSE } \\
\text { CSVH* }\end{array}$ & $\begin{array}{l}\text { BCPECSE } \\
\text { CSC }\end{array}$ & $\begin{array}{l}\text { BCPECSE } \\
\text { CTC }\end{array}$ & $\begin{array}{l}\text { BCPECSE. } \\
2 *\end{array}$ & $\begin{array}{l}B C P \subseteq C S E \\
3\end{array}$ \\
\hline $\begin{array}{l}\text { RCPE } \\
\text { CE }\end{array}$ & $\begin{array}{l}7060 \\
7060\end{array}$ & $\begin{array}{l}7080 \\
7000\end{array}$ & $\begin{array}{l}7065 \\
7020\end{array}$ & $\begin{array}{l}7055 \\
7035\end{array}$ & $\begin{array}{l}7140 \\
6960\end{array}$ & $\begin{array}{l}7130 \\
6970\end{array}$ & $\begin{array}{l}7110 \\
6985\end{array}$ & $\begin{array}{l}7135 \\
6760\end{array}$ & $\begin{array}{l}7045 \\
6985\end{array}$ \\
\hline $\begin{array}{l}C E \\
H\end{array}$ & $\begin{array}{l}360 \\
-30\end{array}$ & $\begin{array}{r}360 \\
-35\end{array}$ & $\begin{array}{l}360 \\
-35\end{array}$ & $\begin{array}{l}360 \\
-35\end{array}$ & $\begin{array}{l}400 \\
-25\end{array}$ & $\begin{array}{r}390 \\
-25\end{array}$ & $\begin{array}{r}38 J \\
-25\end{array}$ & $\begin{array}{r}350 \\
-35\end{array}$ & $\begin{array}{r}375 \\
-35\end{array}$ \\
\hline $\begin{array}{l}B C P E \\
C P F\end{array}$ & $\begin{array}{l}-10 \\
-10\end{array}$ & & & & & & & & \\
\hline $\begin{array}{l}\text { ACPE } \\
\text { CSE-H } \\
\text { RCPE } \\
C S E-C\end{array}$ & & $\begin{array}{r}-25 \\
30\end{array}$ & $\begin{array}{r}-5 \\
10 \\
-60 \\
45\end{array}$ & $\begin{array}{r}-40 \\
25\end{array}$ & & $\begin{array}{r}-40 \\
55\end{array}$ & $\begin{array}{r}-15 \\
35 \\
-65 \\
65\end{array}$ & $\begin{array}{r}-75 \\
10\end{array}$ & \\
\hline $\begin{array}{l}\text { RCPE } \\
\text { CSE }=2\end{array}$ & & & & & 70 & & $\cdot$ & $\begin{array}{r}-75 \\
2.7\end{array}$ & \\
\hline $\begin{array}{l}\text { SCPE } \\
\text { CSE-3 }\end{array}$ & & & & & & & . & & $\begin{array}{l}10 \\
25\end{array}$ \\
\hline
\end{tabular}


TABIE 58. RONO ANO GEMINAL FRAGMENTS ON SECONOARY ETHYNYL CARBONS IN GANANA LMO.S

\begin{tabular}{|c|c|c|c|c|c|c|c|c|c|}
\hline & $\begin{array}{l}\text { RCSECPE } \\
\text { CPH }\end{array}$ & $\begin{array}{l}\text { BC SECPE } \\
\text { CSH }\end{array}$ & $\begin{array}{l}\text { BC SECPE } \\
\text { C.TH }\end{array}$ & $\begin{array}{l}\text { RC SF.CPE } \\
\text { CSVH }\end{array}$ & $\begin{array}{l}\text { BCSECPE } \\
\text { CSC }\end{array}$ & $\begin{array}{l}\text { BCSECPE } \\
\text { CTC }\end{array}$ & $\begin{array}{l}\text { BCSECPE } \\
2 *\end{array}$ & $\begin{array}{l}\text { BCSECPF } \\
3\end{array}$ & $\begin{array}{l}\text { BCSECSE } \\
\mathrm{HH}\end{array}$ \\
\hline $\begin{array}{l}\text { BCSE } \\
\text { CE }\end{array}$ & $\begin{array}{l}7000 \\
7080\end{array}$ & $\begin{array}{l}7020 \\
7065\end{array}$ & $\begin{array}{l}7035 \\
.7055\end{array}$ & $\begin{array}{l}6960 \\
7140\end{array}$ & $\begin{array}{l}6970 \\
7130\end{array}$ & $\begin{array}{l}6985 \\
7110\end{array}$ & $\begin{array}{l}6900 \\
7135\end{array}$ & $\begin{array}{l}6985 \\
7045\end{array}$ & $\begin{array}{l}7020 \\
7020\end{array}$ \\
\hline $\begin{array}{l}\text { CSE } \\
\text { CP }\end{array}$ & $\begin{array}{r}265 \\
40\end{array}$ & & & & & . & & & $\begin{array}{r}270 \\
45\end{array}$ \\
\hline $\begin{array}{l}\text { CSE } \\
\text { CS-H } \\
\text { CSE } \\
\text { CS-C } \\
\text { CSE } \\
\text { CS }-3\end{array}$ & . & $\begin{array}{r}295 \\
55 \\
280 \\
50 \\
290 \\
50\end{array}$ & & & $\begin{array}{r}225 \\
10 \\
205 \\
10 \\
240 \\
20\end{array}$ & & . & & \\
\hline $\begin{array}{l}\text { CSE } \\
\text { CT-HH }\end{array}$ & & & $\begin{array}{r}310 \\
65\end{array}$ & & & $\begin{array}{r}245 \\
20\end{array}$ & & & \\
\hline $\begin{array}{l}\text { CSE } \\
\text { CSV-H }\end{array}$ & & & & $\begin{array}{r}170 \\
0\end{array}$ & & & $\begin{array}{r}310 \\
70\end{array}$ & & \\
\hline $\begin{array}{l}\text { CSE } \\
\text { CSE }\end{array}$ & & & & & & & . & $\begin{array}{r}210 \\
70\end{array}$ & \\
\hline $\begin{array}{l}B C S E \\
C P E-H\end{array}$ & $\begin{array}{r}30 \\
-25\end{array}$ & $\begin{array}{r}10 \\
-5\end{array}$ & & & $\begin{array}{r}55 \\
-40\end{array}$ & $\begin{array}{r}35 \\
-15\end{array}$ & $\begin{array}{r}10 \\
-75\end{array}$ & . & \\
\hline $\begin{array}{l}\text { BCSE } \\
\text { CPE-C } \\
\text { BCSE } \\
\text { CPE-2* }\end{array}$ & . & $\begin{array}{r}45 \\
-60\end{array}$ & $\begin{array}{r}25 \\
-40\end{array}$ & $\begin{array}{r}70 \\
0\end{array}$ & 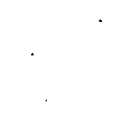 & $\begin{array}{r}65 \\
-35\end{array}$ & $\begin{array}{r}20 \\
-75\end{array}$ & & \\
\hline $\begin{array}{l}B C S F \\
C P E-3\end{array}$ & & & & & & & & $\begin{array}{l}25 \\
10\end{array}$ & \\
\hline $\begin{array}{l}\text { RC.SF } \\
\text { CSE-HH }\end{array}$ & & & & • & & & & & $\begin{array}{l}20 \\
20\end{array}$ \\
\hline
\end{tabular}


the three is that the coefficient changes occurring in the bond fragments when primary carbons are replaced by secondary carbons (and probably tertiary carbons also) are identical for $C-C, C V=C V$ and $C E \equiv C E$ bonds.

On the other hand, the banana bond fragments of triple bonds are very sensitive to vicinal substitution, inasmuch as it makes a difference whether the near carbon of the vicinal bond is primary, secondary or tertiary, which was not the case for the other bond fragments. This introduces an additive correction to the effects of vicinal $\mathrm{CH}$ or $\mathrm{CC}$ substitution discussed previously in Table 46 for the other CC bond fragments. It is presumably also true for triple bonds between secondary carbons. 


\section{ORBITAL GEMINAL FRAGMENTS}

\section{Introduction}

The coefficients for the geminal fragments were included with their adjacent bond fragment coefficients in Tables 36-58 of the preceding chapter. The geminal fragment coefficients are much smaller than the bond fragment coefficients and have widely varying polarities and nodal properties. Furthermore, the coefficients depend not only on the character of the geminal fragment, but also on that of the adjacent bond fragment. If the type of the far (relative to the geminal fragment) hybrid belonging to the adjacent bond fragment is fixed, and the near carbon hybrid varied over either the alkyl, vinyl, ethynyl or banana types, then concomitant geminal fragment coefficient changes are almost independent of the geminal fragment type. Also, geminal fragment coefficients are influenced by vicinal fragments coplanar with the adjacent bond fragment, and by vicinal fragments coplanar with the geminal fragment itself. Given the adjacent bond fragment type and the geminal fragment type, the effects on the coefficients of the geminal fragment from vicinal fragments lying coplanar with the bond fragment and from vicinal fragments lying coplanar with the geminal fragment are simply additive.

The geminal fragment coefficients are not well represented by SMO's and PMO's such as those given previously in Table 35. The latter types of orbitals tend to produce geminal fragment coefficients that have nodes near the center of the bond region. We shall return to these differences in the last chapter of this work. 


\section{Alkyl CH Fragments}

Geminal alkyl CH fragments can be characterized by (1) their associated bond fragment, (2) coplanar fragments vicinal to the geminal fragment, (3) fragment's lying coplanar and vicinal to the bond fragment. When the latter are changed from $\mathrm{CH}$ to $\mathrm{CC}$, systematic changes occur in the geminal fragment coefficients so that geminal fragments adjacent to bond fragments with coplanar vicinal $\mathrm{CH}$ fragments can be chosen as prototypes. They are displayed in Tables 59-61 which contain geminal $\mathrm{CH}$ fragments with primary, secondary and tertiary carbons, respectively. The columns correspond to various bond fragments with coplanar vicinal $\mathrm{CH}$, the rows correspond to geminal $\mathrm{CH}$ fragments characterized by particular vicinal coplanar bonds.

The variation of the coefficients is very similar in the three tables: the difference between two entries (defined by bond fragment and geminal fragment) in one of the tables is generally close to the corresponding differences in the other tables (provided both entries exist in the tables in question). Within one table characteristically distinct behavior is observed for the following groups of bond fragments: (1) $\mathrm{CH}$, (2) $c-c($ alkyl), (3) $c-c($ vinyl), (4) C-C(ethynyl). A notable distinction between fragments geminal to $\mathrm{CH}$ bonds (group (1)) and the others is that the former have a node near the middle of the fragment, whereas in the latter, the node is towards the atom farthest from the bond fragment.

Tables 62-65 contain the relative changes in the geminal $\mathrm{CH}$ due to substitution trans to the bond fragment, e.g. the first and second rows of column one in Table 65 display the numbers which, when added to the 


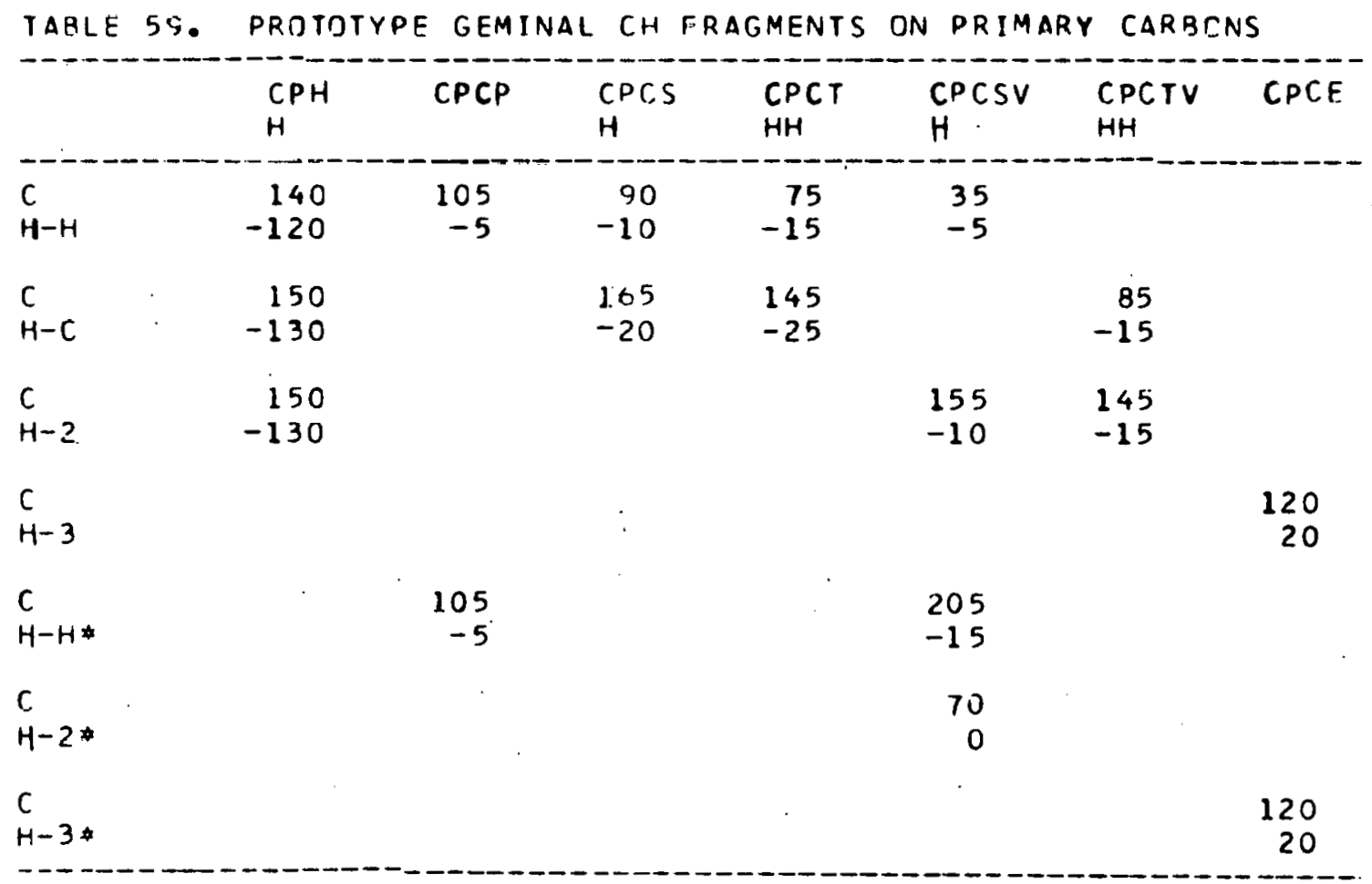




\begin{tabular}{|c|c|c|c|c|c|c|}
\hline & $\begin{array}{l}\mathrm{CSH} \\
\mathrm{HH}\end{array}$ & $\begin{array}{l}\text { CSCP } \\
H\end{array}$ & $\begin{array}{l}\text { CSCS } \\
\mathrm{HH}\end{array}$ & $\begin{array}{l}\text { CSCT } \\
\text { HHH }\end{array}$ & $\begin{array}{l}\text { CSCSV } \\
H H\end{array}$ & $\begin{array}{l}\text { CSCE } \\
H\end{array}$ \\
\hline $\begin{array}{l}\mathrm{C} \\
\mathrm{H}-\mathrm{H}\end{array}$ & $\begin{array}{r}170 \\
-135\end{array}$ & $\begin{array}{l}140 \\
-25\end{array}$ & $\begin{array}{l}125 \\
-25\end{array}$ & $\begin{array}{r}110 \\
-35\end{array}$ & $\begin{array}{r}75 \\
-25\end{array}$ & \\
\hline $\begin{array}{l}\mathrm{C} \\
\mathrm{H}-\mathrm{C}\end{array}$ & $\begin{array}{r}180 \\
-145\end{array}$ & & & $\begin{array}{r}175 \\
-40\end{array}$ & & 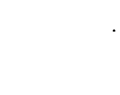 \\
\hline $\begin{array}{l}C \\
H-2\end{array}$ & $\begin{array}{r}195 \\
-150\end{array}$ & $\begin{array}{r}145 \\
-35\end{array}$ & $\begin{array}{l}130 \\
-40\end{array}$ & & $\begin{array}{r}195 \\
-25\end{array}$ & \\
\hline $\begin{array}{l}\mathrm{C} \\
\mathrm{H}-3\end{array}$ & & & . & & & $\begin{array}{r}1 \in 0 \\
0\end{array}$ \\
\hline$\stackrel{C}{H-23}$ & & & & . & : & $\begin{array}{r}165 \\
-10\end{array}$ \\
\hline $\begin{array}{l}\mathrm{C} \\
\mathrm{H}-2 \text { * }\end{array}$ & & & & & $\begin{array}{r}185 \\
-25\end{array}$ & \\
\hline $\begin{array}{l}\mathrm{C} \\
\mathrm{H}-22 \text { * }\end{array}$ & & & & & $\begin{array}{r}110 \\
-30\end{array}$ & \\
\hline
\end{tabular}


TABLE 61. PROTOTYPE GEMINAL CH FRAGNENTS ON TERTIARY CARBONS

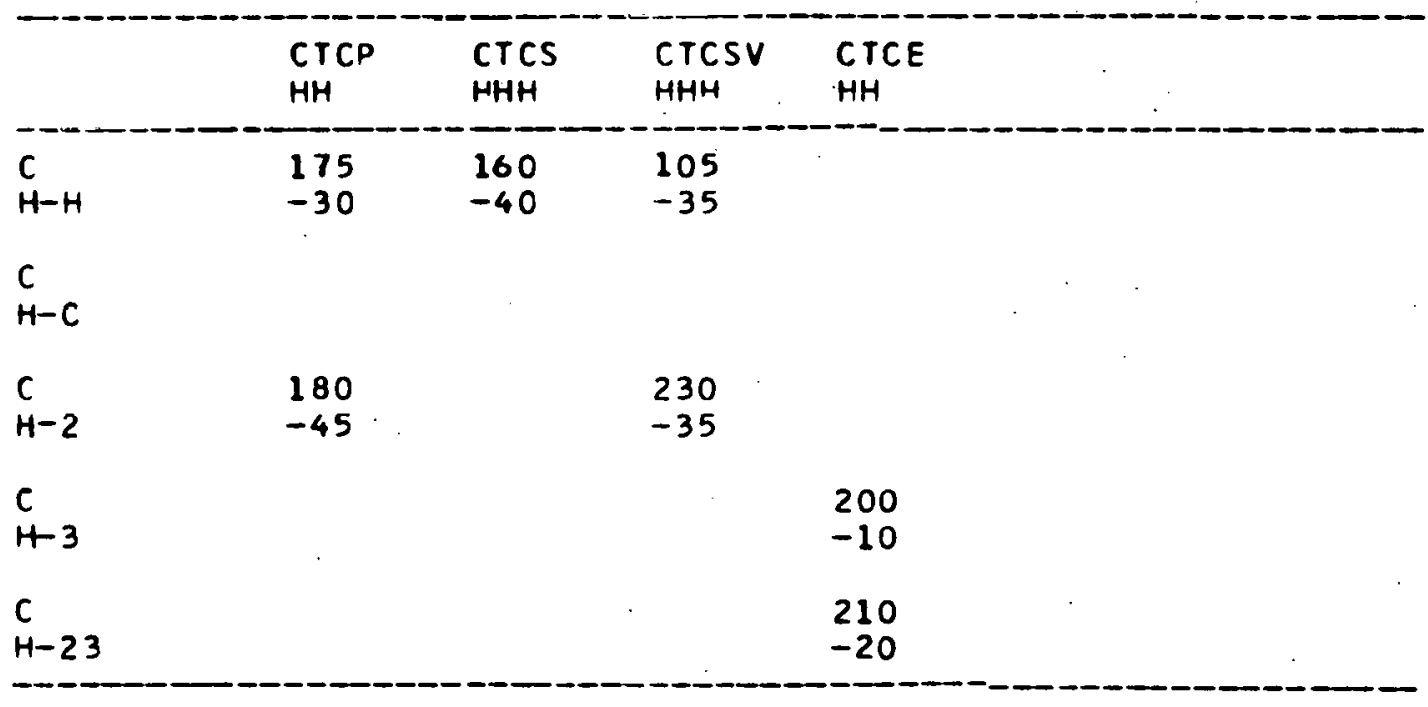




\begin{tabular}{|c|c|c|c|c|c|c|c|c|c|c|}
\hline & $\begin{array}{l}C P H \\
C\end{array}$ & $\mathrm{CSH}^{\mathrm{CSH}}$ & $\begin{array}{l}\text { CPCS } \\
C\end{array}$ & $\begin{array}{l}\mathrm{CPCT} \\
\mathrm{C}\end{array}$ & $\begin{array}{l}\text { CPCSV } \\
C\end{array}$ & $\begin{array}{l}\text { CSCP } \\
C\end{array}$ & ${ }_{r}^{\csc s}$ & ${ }_{c}^{\operatorname{cscs} v}$ & $\underset{C}{\text { CSCE }}$ & $\begin{array}{l}C T C P \\
C\end{array}$ \\
\hline$r$ & $\begin{array}{r}0 \\
-10\end{array}$ & $\begin{array}{r}0 \\
-10\end{array}$ & $\begin{array}{l}0 \\
0\end{array}$ & $\begin{array}{r}-5 \\
0\end{array}$ & $\begin{array}{l}0 \\
0\end{array}$ & $\begin{array}{r}0 \\
-5\end{array}$ & $\begin{array}{r}0 \\
-5\end{array}$ & & & $\begin{array}{r}5 \\
-10\end{array}$ \\
\hline $\begin{array}{l}\mathrm{C} \\
\mathrm{H}-\mathrm{C}\end{array}$ & $\begin{array}{r}0 \\
-10\end{array}$ & & $\begin{array}{r}10 \\
0\end{array}$ & $\begin{array}{l}5 \\
0\end{array}$ & & $\begin{array}{r}5 \\
-10\end{array}$ & & & & \\
\hline$\stackrel{f}{H-2}$ & & & & & . & & & $\begin{array}{l}10 \\
-5\end{array}$ & & \\
\hline$\stackrel{r}{H-3}$ & & & & & & & & & $\begin{array}{r}0 \\
-5\end{array}$ & \\
\hline
\end{tabular}


TABLE 63. SUSSTITUEAT EFFECTS IN GEMINAL CH FRAGMENTS WITH THE INTROOUCTION OF A DOUBLE BONO

\begin{tabular}{|c|c|c|c|c|c|c|}
\hline & $\begin{array}{l}C P H \\
2\end{array}$ & ${ }_{2}^{\mathrm{CSH}}$ & $\begin{array}{l}\text { CPCS } \\
2\end{array}$ & $\begin{array}{l}C P C T \\
2\end{array}$ & $\begin{array}{l}\operatorname{CSC} \theta \\
2\end{array}$ & $\begin{array}{l}\text { CICP } \\
2\end{array}$ \\
\hline $\begin{array}{l}\mathrm{C} \\
\mathrm{H}-\mathrm{H}\end{array}$ & $\begin{array}{r}0 \\
-10\end{array}$ & $\begin{array}{r}0 \\
-1 C\end{array}$ & $\begin{array}{l}0 \\
0\end{array}$ & $\begin{array}{r}-5 \\
0\end{array}$ & $\begin{array}{l}5 \\
0\end{array}$ & $\begin{array}{r}0 \\
-10\end{array}$ \\
\hline${ }_{1+C}^{r}$ & $\begin{array}{l}-5 \\
-5\end{array}$ & & $\begin{array}{r}-5 \\
0\end{array}$ & $\begin{array}{l}0 \\
0\end{array}$ & & \\
\hline $\begin{array}{l}C \\
4-2\end{array}$ & $\begin{array}{r}-15 \\
4 C\end{array}$ & $\begin{array}{r}-15 \\
50\end{array}$ & & & $\begin{array}{r}-15 \\
30\end{array}$ & $\begin{array}{r}-20 \\
40\end{array}$ \\
\hline
\end{tabular}


TARLE 64. SUBSTITUENT EFFECTS IN GEMINAL CH FRAGMENTS WITH THE INTRODUCTION OF A TRIPLF BOND

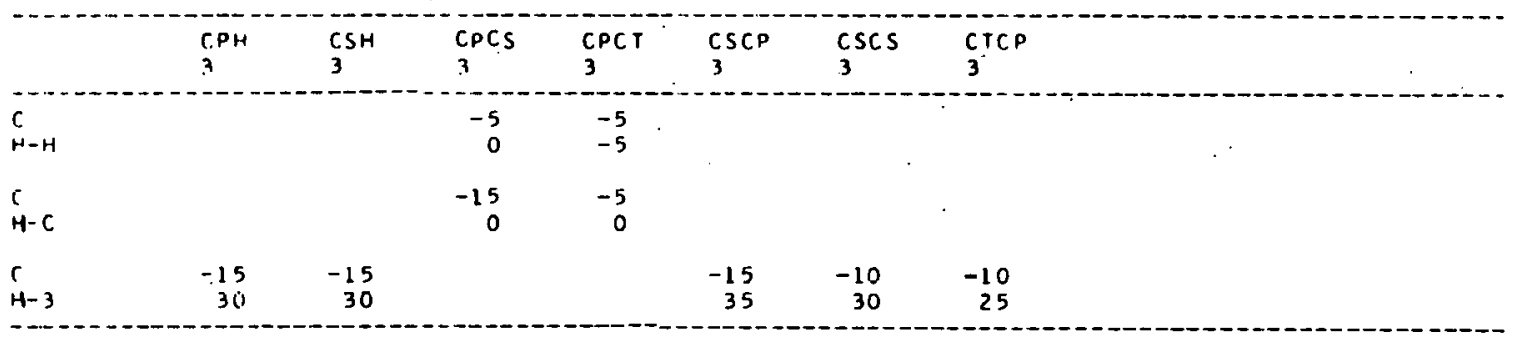


TAALE O5. SUASTITUEAT EFFECTS IN GEMINAL CH FRAGMENIS MITH THE INTRODUCTION OF DOUBLE AND TRIPLE BOACS

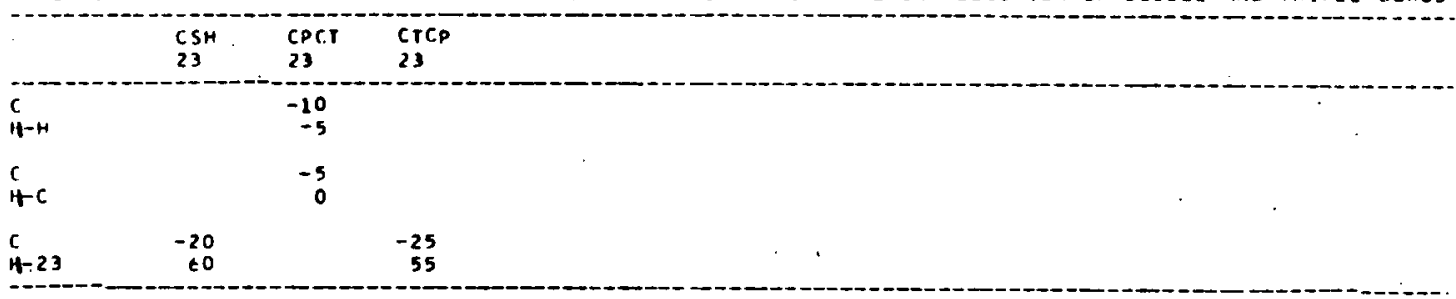


$\mathrm{CH}-\mathrm{H}$ geminal fragment in the $\mathrm{CPH}-\mathrm{H}$ prototype, give the $\mathrm{CH}-\mathrm{H}$ adjacent to the $\mathrm{CPH}-\mathrm{C}$. Exceptions are the $\mathrm{CH}-3$ and $\mathrm{CH}-23$ of Tables 64 and 65 , which are given relative to the $\mathrm{CH}-2$ fragments of the prototypes, there being no prototype $\mathrm{CH}-3$ or $\mathrm{CH}-23$. On the whole, the geminal fragments are only siightly affected by these substitutions, and what changes do occur are fairly regular. The geminal $\mathrm{CH}-2$ adjacent to the $\mathrm{CPH}-2, \mathrm{CSH}-2$, etc. in Table 63 are the only ones to change appreciably, and all by about the same amount. Thus, the deductions made for the prototypes also hold in general and, except for the $\mathrm{CH}-2$ just mentioned, the geminal fragments are little changed by substitution trans to the bond fragment.

The additivity of substituent effects follows, e.g. from Table 61. Calculating the differences between the CTH-HH2 (adjacent to the CTCP-H2) and $\mathrm{CTH}-\mathrm{HHH}$ (adjacent to the CTCP-HH), and similarly for the CTH-HH3 (adjacent to the $(T C P-H 3)$, one finds that addition of these two to the CTH-HHH approximately gives the CTH-23 (adjacent to the CTCP-23).

Some coefficient changes due to $H \rightarrow C$ substitution in the cis position are shown in the first six rows of Table 66, relative to the coefficients of the $\mathrm{CH}-\mathrm{H} \%$ fragment geminal to the $\mathrm{CPH}-\mathrm{H} *$ bond. ( $1 \mathrm{t}$ is interesting that the latter are the same as for the trans case). Although the values for the $\mathrm{CH}-\mathrm{H} *$ and $\mathrm{CH}-2 \%$ cases are approximately reversed, the changes from the corresponding trans cases are small. This also holds for CH geminal. fragments adjacent to CSH bonds. The changes are considerably larger for geminal fragments adjacent to $C C$ bond fragments along axes of hindered rotation (except for ethane). 


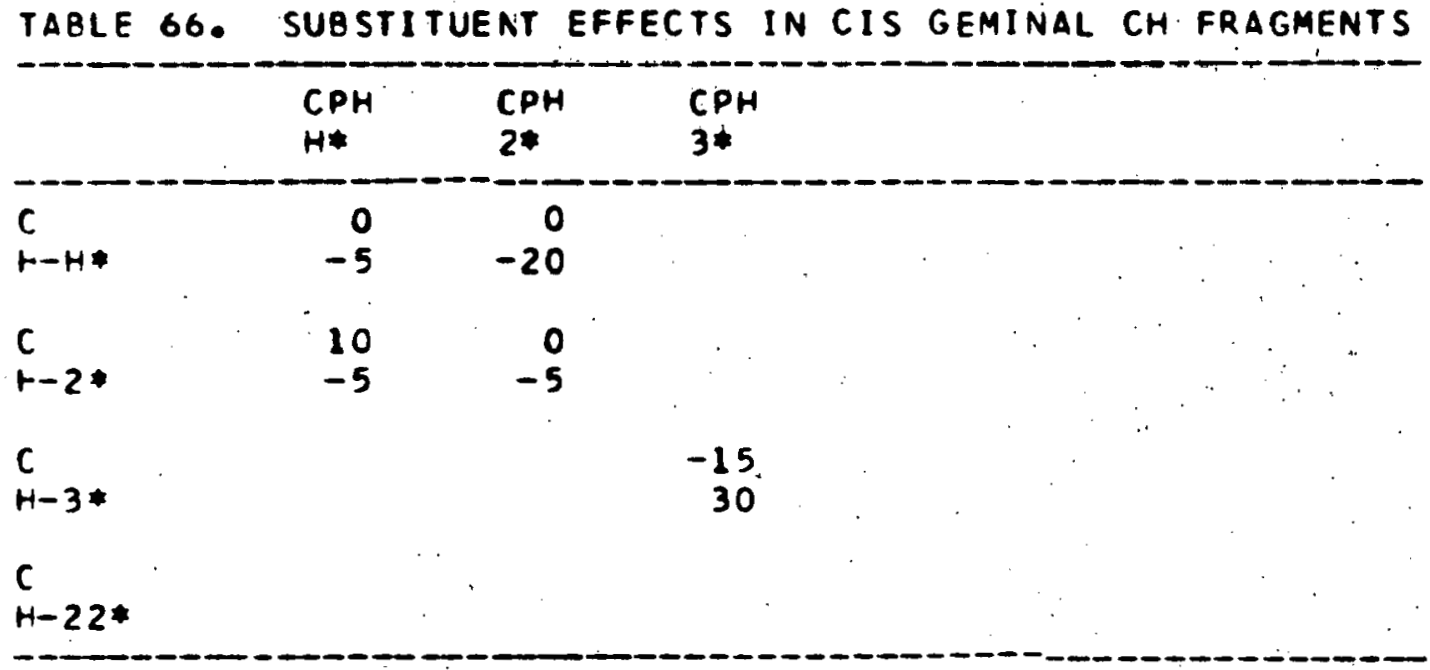




\section{Alkyl CC Fragments}

The CC prototypes (having CH fragments coplanar and vicinal to their bond fragments) are shown in Tables 67 and 68 . Again it can be seen that coefficient changes due to changes in the bond fragment are almost the same for geminal CC fragments centered on secondary and tertiary carbons (the primary carbon series has only one member). Regularities for substitutions trans to the geminal fragment are also observed among those adjacent to corresponding bond fragments (e.g. CPH, CSH, and CTH, or CSCP and CTCP, etc.). However, the geminal fragments CC differ from the (geminal) $\mathrm{CH}$ fragments in that no group regularities such as discussed with Tables 59-61 are observed. Also, the geminal CC coefficients are usually opposite in sign to the geminal $\mathrm{CH}$ and smaller by a factor of two or three.

The effects of substitution trans to the bond fragment are shown in Tables 69 and 70. Here, because of a smaller sampling, a better perspective is obtained by listing the primary, secondary and tertiary together, which is permissable since the corresponding values never differ by more than 0.0010 (a fact also true of the geminal $\mathrm{CH}$ ). The relative quantities have the same meaning as for the $\mathrm{CH}$ geminal fragments with two exceptions: the CCE-H are taken relative to the prototype's CCSV-H; and the geminal CCS of the CCP-C bond are taken relative to its geminal CSCS-H. Each group of rows labelling the same geminal fragment - e.g., the rows $C C P-H, C C P-C$, $C C P-2, C C P-3$ and $C C P-23$ - is as regular as was the case for the $C H$ geminal fragments. However, the magnitude of the effect is slightly larger for the CC geminal fragments. 


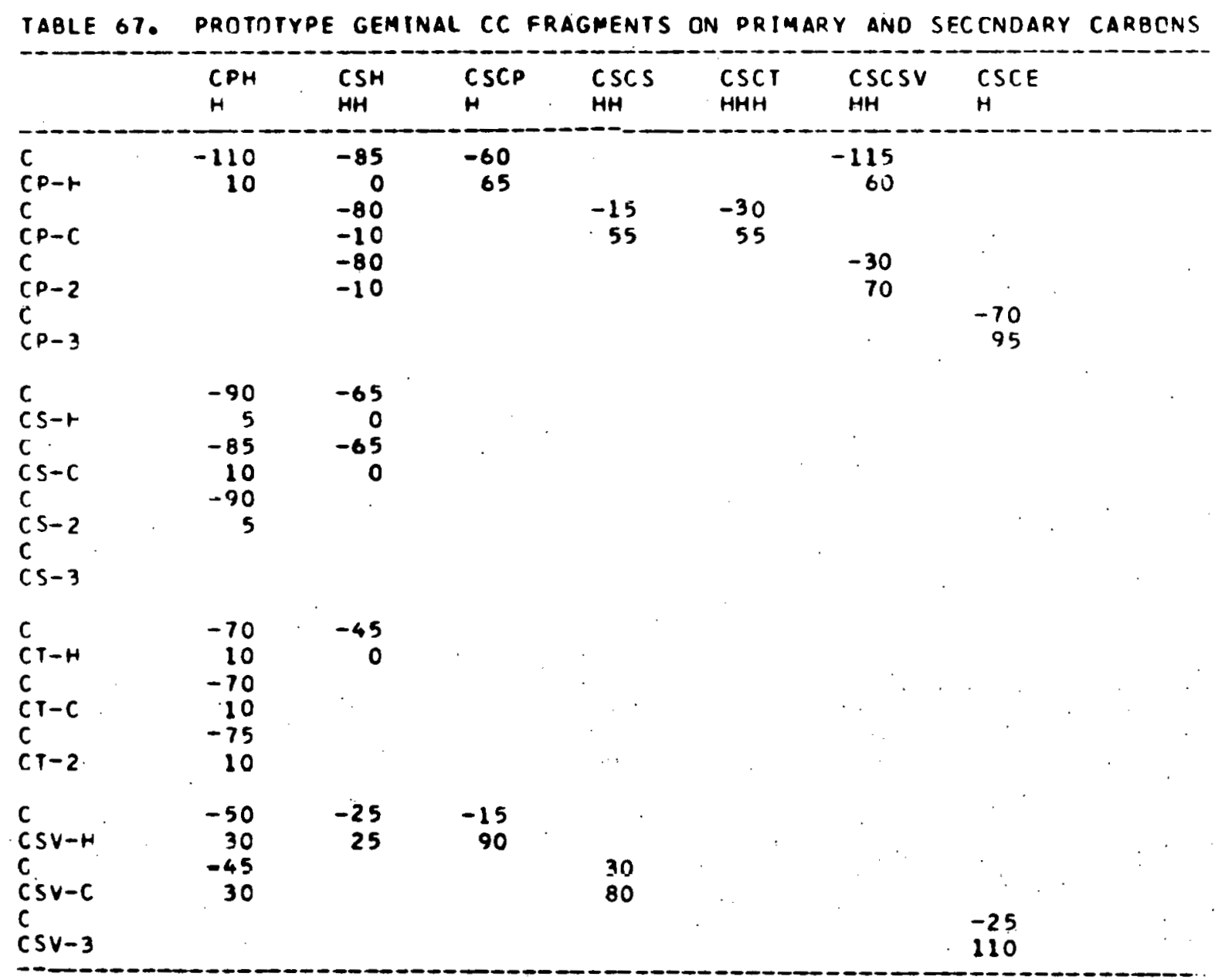


TABLE 68. PROTOTYPE TERTIARY GEMINAL CC FRAGMENTS

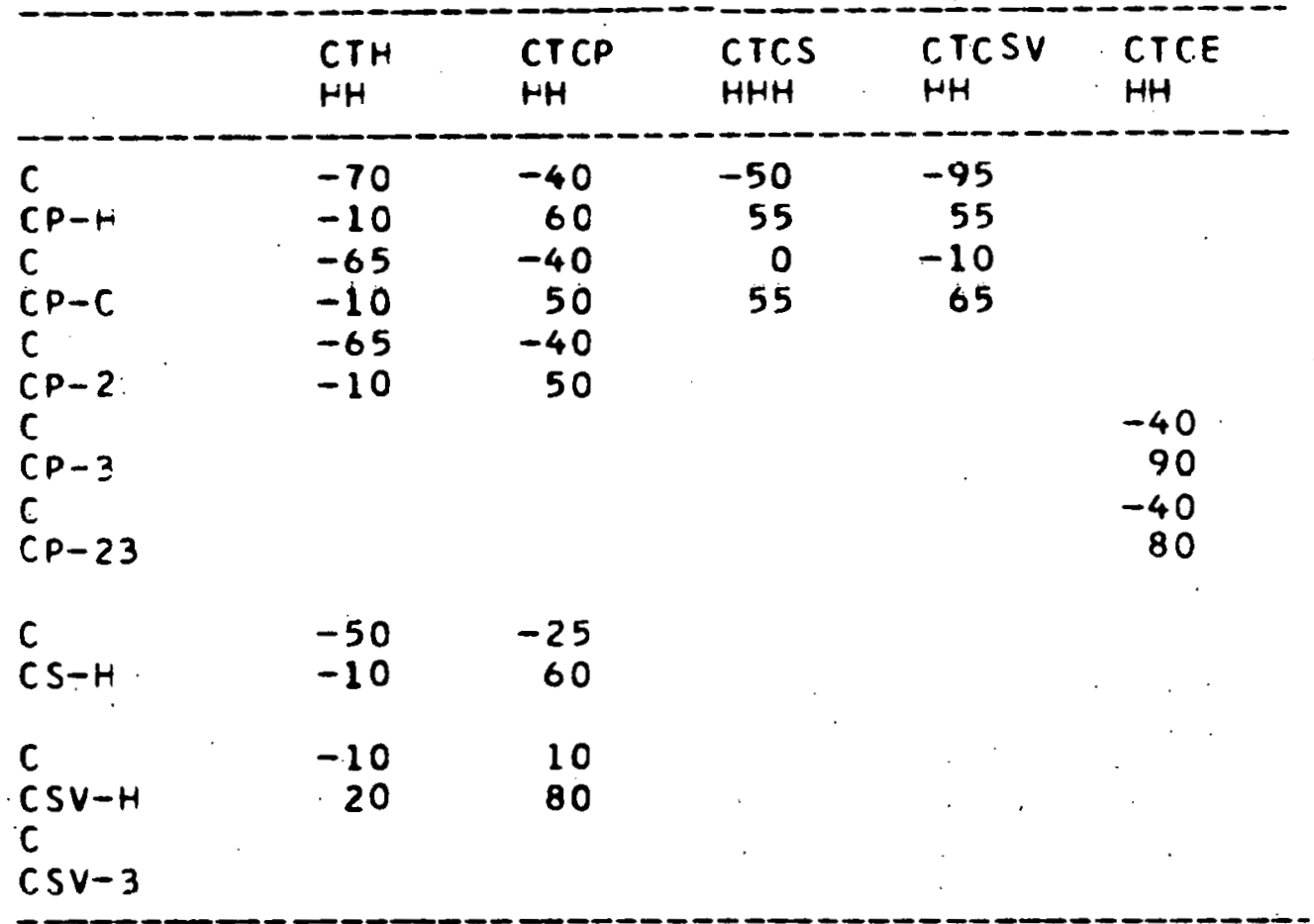


IABLE 69. SUBSTITUFAT. EFFECTS IN ALKYL GEMINAL CC FRAGMENTS HITH THE INTRODUCTION OF CC BONOS

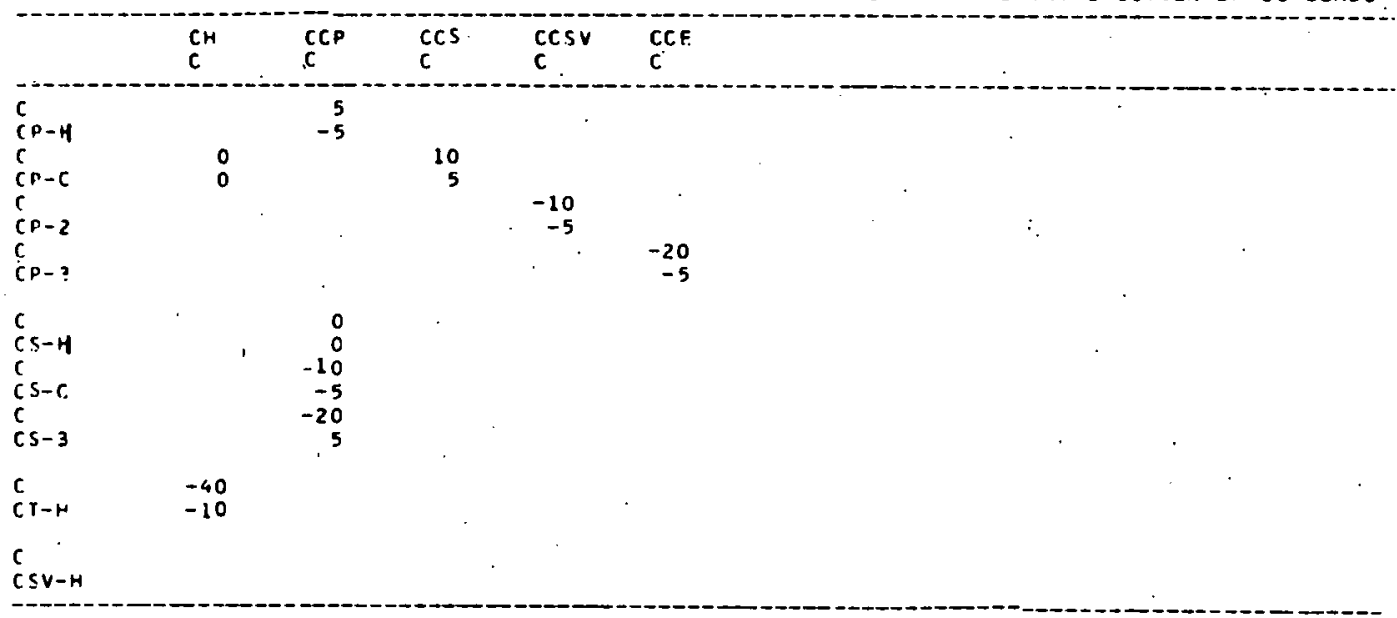


IAHLF 10. SUHSTITUENT EFFECTS IN ALKYL GEMINAL CC FRAGMENTS WITH THE INTRDOUCTION OF aANANA BCNDS

\begin{tabular}{|c|c|c|c|c|c|c|c|c|c|c|}
\hline & ${ }_{i}^{C H}$ & ${ }_{2}^{C C P}$ & ${ }_{3}^{C H}$ & ${ }_{3}^{C C . P}$ & $c_{3}^{\operatorname{css}}$ & $\begin{array}{l}\operatorname{crsv} v \\
3\end{array}$ & $\begin{array}{l}\text { CCE } \\
3\end{array}$ & $\begin{array}{l}\mathrm{CH} \\
23\end{array}$ & $\begin{array}{l}C C P \\
23\end{array}$ & $\begin{array}{l}\mathrm{CH} \\
33\end{array}$ \\
\hline $\begin{array}{l}C \\
C P-H \\
C \\
C P-C \\
C \\
C P-2 \\
C \\
C P-3 \\
C P \\
C P-23\end{array}$ & $\begin{array}{r}0 \\
0 \\
-5 \\
10 \\
10 \\
40\end{array}$ & $\begin{array}{r}0 \\
0 \\
5 \\
35\end{array}$ & $\begin{array}{l}-10 \\
-10\end{array}$ & 30 & $\begin{array}{r}-5 \\
5\end{array}$ & $\begin{array}{r}0 \\
20 \\
0 \\
25\end{array}$ & & $10^{5}$ & & . \\
\hline $\begin{array}{l}c \\
c s-n \\
c \\
c s-3\end{array}$ & $\begin{array}{l}0 \\
0\end{array}$ & & $\begin{array}{r}0 \\
20\end{array}$ & & & & & & & \\
\hline $\begin{array}{l}c \\
C S V-h \\
c \\
\operatorname{csv}-3\end{array}$ & $\begin{array}{r}-110 \\
-35\end{array}$ & & . & & & & & $\because \begin{array}{r}-110 \\
20\end{array}$ & $\begin{array}{r}-80 \\
5\end{array}$ & \\
\hline $\begin{array}{l}C \\
C E-H \\
C \\
C E-C \\
C \\
C, E-3\end{array}$ & & & $\begin{array}{r}-125 \\
5 \\
-125 \\
-5\end{array}$ & $\begin{array}{r}-110 \\
25\end{array}$ & $\begin{array}{r}-100 \\
30,\end{array}$ & & $\begin{array}{r}-100 \\
30\end{array}$ & $\begin{array}{r}-125 \\
5\end{array}$ & $\begin{array}{r}-110 \\
20\end{array}$ & $\begin{array}{r}- \\
-125 \\
25\end{array}$ \\
\hline
\end{tabular}


Again with the exception of ethane, the geminal CC fragments which lie along axes of hindered rotation (see Tables 36 and 44 ), or are adjacent to them (Table 44), change noticeably from the cis to the trans confirmations. In this respect they are directly analogous to the $\mathrm{CH}$ geminal fragments.

Finally, the additivity of the substituents also obtains for the $C C$, as can be seen from the CTCP in Table 68.

\section{Primary Vinyl $\mathrm{CH}$ Fragments}

The primary vinyl CH geminal fragments form two markediy different classes: (1) the CPVH fragments adjacent to CPVH bond fragments and (2) the CPVH fragments adjacent to banana bond fragments. In each case, the contributions from the carbon (near) hybrids are two to three times larger than was the case for the previously discussed alkyl geminal fragments.

The CPVH geminal fragments adjacent to CPVH bond fragments were included in Tables 47-9. Those whose CPVH bond fragments are cis to the vicinal monosubstituent (Table 47) are about 10\% larger than those adjacent to bond fragments trans to the vicinal. monosubstituent (Table 48). Both show two classes whose members have nearly equal coefficients: (1) XNFN, XTT and XPL, (2) XCT, XCC and XCP, with X $=$ C (Table 47) or T (Table 48)。 Ethylene differs from all of these in that the contribution from its hydrogen 15 is smaller, although its carbon hybrid coefficient is identical to those of class (2) in Table 48.

Only members of the first class (the XNFN, XTT and XPL) are represented for the CPVH geminal fragments adjacent to CPVH bond fragments of 
disubstituted ethylenes in Table 49. These are found to closely resemble the class ( 1 ) coefficients of Table 47 (bond fragment cis to the monosubstituent), although the latter have slightly smaller hydrogen orbital coefficients.

The CPVH geminal fragments adjacent to banana bond fragments were given in Tables 53 and 54. They are nodeless. In this way they differ from the CPVH geminal fragments adjacent to CPVH bond fragments, which have pronounced nodes towards the hydrogen atoms. They form basically two classes: (1) $\mathrm{CPVH}$ geminal fragments which have a $\mathrm{CH}$ fragment in the trans positions, and (2) the CPVH geminal fragments which have a CC fragment in the cis positions. Within each class the carbon and hydrogen hybrid coefficients separately have pretty much the same magnitude. Within the second class the vinyl carbon of the bond fragment that is farthest from the geminal fragment can be tertiary or secondary. In the latter, a finer distinction into two subclasses can be observed, corresponding to the two cases that the banana bond fragment has in trans positions (1) a $\mathrm{CC}$ or double bond, (2) a $\mathrm{CH}$ or triple bond.

\section{Secondary Vinyl CH Fragments}

The secondary vinyl $\mathrm{CH}$ geminal fragments adjacent to CSVC single bond fragments (Tables 51-2) noticeably resemble the CPVH geminal fragments adjacent to CPVH single bond fragments. Their coefficients are slight ly larger when the bond fragment's carbon atom farthest from the geminal fragment is alkyl (Table 51) then when it is vinyl or ethynyl (Table 52). The variations in these secondary vinyl $\mathrm{CH}$ geminal fragments show 
regularities similar to those previously observed for alkyl geminal fragments. Also, the difference between the geminal fragment coefficients of two enties in Table 51 is close to the difference between the corresponding entries in Table 52 (if such corresponding entries exist). However, the CSVH geminal fragments are insensitive to the orientation of their coplanar vicinal fragments.

The CSVH geminal fragments adjacent to banana bond fragments are shown in Table 54. The relations between them and the CSVH geminal fragments just discussed are very similar to the relations between the two analogous CPVH geminal fragments considered in the preceding section. As in the preceding paragraph, the regularities here are like those of alkyl fragments. There is also an insensitivity to the orientations of the vicinal fragments coplanar with the geminal fragments. Although data are not available for the BCSVCSV and BCSVCTV bond fragments, it is likely that they behave similarly.

\section{Single Bond Vinyl CC Fragments}

Like the vinyl CH geminal fragments just discussed, the single bond vinyl CC geminal fragments fall into two clearly distinct classes: the single bond $C C$ geminal fragments adjacent to single bond fragments and (2) the single bond CC geminal fragments adjacent to banana bond fragments.

The fragments in the first class have nodes near the center of the bond region, which is quite different from the alkyl. CC geminal fragments. A further distinction is possible in this case: the CC geminal fragments 


\section{6}

adjacent to $\mathrm{CH}$ bond fragments (Table 50) differ from those adjacent to CC bond fragments (Tables 51-2). The greatest variations in the coefficients of the former (Table 50) result from changes in the character of the far carbon atom of the geminal fragment and appear to be independent of the nature of vicinal fragments coplanar with the bond or vicinal fragments. Smaller regular coefficient variations are induced by changes in the vicinal fragments coplanar with the bond fragments. The smallest coefficient variations accompany changes in the vicinal fragments coplanar with the CC geminal fragment itself. Although the sampling of the vinyl CC geminal fragments is limited, the two entries in Table 51 differ from their counterparts adjacent to $\mathrm{CH}$ bond fragments (Table 50) by the same amount. This suggests that the same coefficient variations occur in all vinyl ce geminal fragments adjacent to single bond fragments.

The coefficients of the $C C$ single bond geminal fragments adjacent to banana bond fragments (Tables 54-5) are all positive, in sharp contrast with those adjacent to single bonds. In addition, the coefficients of the near hybrid are several times larger than those of the far hybrid. Both features parallel the differences recorded between the vinyl $\mathrm{CH}$ geminal fragments adjacent to single bond fragments and the vinyl $\mathrm{CH}$ geminal fragments adjacent to banana bond fragments. The observations made for the coefficient variations in the geminal fragments adjacent to CC single bond fragments also apply to the geminal fragments adjacent to banana bond fragments.

The banana bond geminal fragments adjacent to vinyl CC single bond fragments (Tables 51-2) have far hybrid coefficients that are about one 
half of the far hybrid coefficients found for those adjacent to $\mathrm{CH}$ bond fragments. As in the latter case, all of their coefficients are always negative. The highly regular coefficient trends in Tables 51 and 52 are very sensitive to the substituent which is vicinal and coplanar with the banana geminal fragment. There is also a large dependence on the symmetry or asymmetry of the noncoplanar vicinal fragments (see, e.g. BCVCPV-H and BCVCPV-HA in Table 51 and Figure 2). Superimposed on these effects are smaller coefficient dependences on the vicinal fragments coplanar with the adjacent vinyl CC bond fragment. These latter appear to be the same in Tables 51 and 52 .

\section{Double Bond Fragments}

The banana geminal fragments form three spectacularly different classes: (1) those adjacent to vinyl $\mathrm{CH}$ bond fragments, (2) those adjacent to vinyl CC single bond fragments, and (3) those adjacent to banana bond fragments. We shall discuss them in turn.

The coefficients for banana geminal fragments adjacent to vinyl $\mathrm{CH}$ bond fragments are always negative. Furthermore, the magnitudes of the near hybrid coefficients are always about twice those of the far hybrids. The adjacent bond fragment can be used to separate these banana geminal fragments into four classes, corresponding to the cases that the vinyl $\mathrm{CH}$ bond fragment is (1) primary and cis to its vicinal monosubstituent (Table 47); (2) primary and trans to its vicinal monosubstituent (Table 48); (3) primary in disubstituted ethylenes; and (4) secondary (Table 50). Ethylene (not included in this classification) is similar to some of those 
in cases (3) and (4). In class (1), all banana geminal fragments have virtually the same value. In class (2), they differ slightly, depending on their coplanar vicinal fragments (these differences are however independent of the monosubstituent trans to the bond fragment). In class (3), the trends are determined by the substituent lying trans to the adjacent CPVH bond fragments. In class (4), the geminal fragment coefficients show very regular variations which are induced by the vicinal fragments (belonging to the alkyl chain on the same side of the double bond as the $\mathrm{CH}$ bond fragment) that are coplanar with the adjacent bond fragments and the vicinal fragments coplanar with the banana geminal fragment itself. These variations are of similar magnitude and of the type previously discussed.

The coefficients of banana geminal fragments that are adjacent to banana bond fragments (Tables 53-5) are the smallest of all. Most of these coefficients are rather homopolar. Their nodal properties are completely characterized by the statements: (1) coefficients on primary vinyl carbons (Tables 53-4) are always negative, and (2) coefficients on secondary (Table 55) or on tertiary (Table 54) vinyl carbons are always positive. In each class, small regular coefficient variations occur that are induced by vicinal substitution coplanar with the adjacent bond fragment, and by vicinal substitution coplanar with the geminal fragment itself. The latter differ slightly from class to class.

\section{Ethynyl CH Fragments}

Ethynyl $\mathrm{CH}$ geminal fragments (Table 57) can only occur adjacent to primary ethynyl triple banana bond fragments. Their near hybrid 
coefficients are among the largest geminal fragment coefficients found. They all have siight nodes toward the atom farthest from the adjacent bond fragment. Ethynyl $\mathrm{CH}$ geminal fragments form five slightly different coefficient classes according to whether the vicinal fragment coplanar with their adjacent bond fragment is (1) an alky $\mathrm{CH}$ bond, (2) a CC bond, (3) a double bond, (4) a triple bond, and (5) a secondary vinyl $\mathrm{CH}$ bond. The $\mathrm{CH}$ geminal fragment coefficients for acetylene are the same as those of class (1).

\section{Single Bond Ethynyl CC Fragments}

The ethynyl CC single bond geminal fragments can only occur adjacent to secondary ethynyl triple bond fragments. Like the ethynyl $\mathrm{CH}$ geminal fragments, their coefficients are strongly polarized toward the near (relative to the bond fragment) carbon atom. These near hybrid coefficients are several times larger than any of the other CC single bond geminal fragment coefficients encountered. Variations among the single bond ethynyl geminal fragment coefficients are primarily characterized by (1) their far hybrid carbon atom, and (2) the vicinal fragment coplanar with the adjacent triple bond fragment. Generally smaller coefficient changes are induced by vicinal fragments on the same side of the triple bond as, and coplanar with, the geminal fragment itself.

\section{Triple Bond Fragments}

As was the case for the double bond geminal fragments, the triple bond geminal fragment coefficients form three classes: (1) those adjacent to ethynyl CH bond fragments, (2) those adjacent to ethynyl CC single bond 
fragments, and (3) those adjacent to banana bond fragments.

There is a characteristic of triple bond geminal fragment coefficients adjacent to single bond fragments (Table 56) which distinguishes them from all others: the far hybrid coefficient magnitudes are much larger than those of the near hybrid. The banana geminal fragment coefficients adjacent to $\mathrm{CH}$ bond fragments are virtually insensitive to any influences. There are, however, two prominent effects on the near hybrid coefficients of the banana geminal fragments adjacent to CC bond fragments: (1) the farthest carbon atom in the adjacent bond fragment, and (2) the vicinal fragment coplanar with, and on the same side of the triple bond as, the geminal fragment itself.

The coefficients for the triple bond geminal fragments adjacent to triple bond fragments (Tables 57-8) are the smallest of the ethynyl geminal fragments, just as their counterparts were the smallest of the vinyl fragments. Most have nodes near the center of the bond region. They can be characterized by (1) the vicinal fragment coplanar with the adjacent triple bond fragment, and (2) the vicinal fragment coplanar with the geminal. fragment itself. 


\section{ORBITAL VICINAL FRAGMENTS}

\section{Vicinal Groups}

The term 'vicinal group' refers, to the collection of vicinal fragments whose near (relative to the bond fragment) hybrids are located on the same carbon atom. Members of vicinal groups are classified as (1) overflow fragments, those vicinal and coplanar with the bond fragment, or (2) gauche fragments, those vicinal and noncoplanar with the bond fragment. The character of the overflow fragment will also be used to characterize the vicinal group itself.

Overflow and gauche fragment coefficients for a particular vicinal group will appear together in the tables, always listed in the order, overflow fragment first (usually in the first two rows of the table), and the several possible gauche fragments following. of the two coefficients for each fragment, the near (relative to the bond fragment) hybrid will be listed first.

\section{Nodal Properties and Coefficient Magnitudes}

The two coefficients of a particular vicinal fragment have similar magnitudes and opposite signs. If the overflow fragment is trans to the bond fragment, its near hybrid coefficient is always negative (and its far hybrid coefficient always positive). Its adjacent gauche fragment coefficients are several times smaller, with near hybrid coefficients that are always positive (and far hybrid coefficients always negative). If the overflow fragment is cis to the bond fragment, all coefficient signs are the reverse of the trans case. Also, the cis overflow fragment coefficient 
magnitudes are about twice those of its adjacent gauche fragments. These facts have proved important for understanding the origins of hindered rotation $(18,19,68,70)$.

Comparison with Geminal Fragments

As a class, vicinal fragment coefficients differ from those of geminal fragments in two interesting ways:

1. Their nodal properties and coefficient magnitudes are much less varied.

2. Their coefficient magnitudes are larger.

The latter seems all the more noteworthy since the vicinal fragments are farther removed from the bond fragment.

\section{Alkyl Vicinal Groups}

Alkyl vicinal groups are those whose near (relative to the bond fragment) carbon atom is an alkyl carbon. Their coefficients can be grouped according to whether their overflow fragment is $\mathrm{CH}$ or $\mathrm{CC}$. In either case, coefficients for their vicinal fragments form four classes, according to whether their associated bond fragment is (1) $\mathrm{CH}$, (2) $\mathrm{CC}$, (3) double banana or (4) triple banana. Coefficients for a particular vicinal fragment are primarily determined in each category by characterizing both atoms of the bond fragment as either (1) alkyl carbon, (2) vinyl carbon, (3) ethynyl carbon, or (4) hydrogen. Vicinal fragment coefficients whose near hybrids are located on primary carbon atoms differ from corresponding fragment coefficients whose near hybrids are located on secondary or tertiary carbon atoms by amounts: that are nearly independent of the character of the 
bond fragment.

Alkyl CH groups

The coefficients for the primary alkyl $\mathrm{CH}$ groups vicinal to $\mathrm{CH}$ bond fragments are shown in Table 71 (those of the overflow fragment appear in the first two columns). All overflow fragments lie coplanar with $\mathrm{CH}$ bond fragments, but the gauche fragment coefficients display variations that are induced by (1) their coplanar vicinal bonds, which lie adjacent to the bond fragment (the CPH-C columns in Table 71), and (2) coplanar bond chains beyond their coplanar vlcinal bonds (the TT-CPH-C columns in Table 71).

Coefficients for secondary alkyl groups vicinal to $\mathrm{CH}$ bond fragments are listed in Tables 72-3. Regular variations in the gauche fragments are induced as: for the primary $\mathrm{CH}$ vicinal fragments. Now, however, the fragments and their associated chains need not be adjacent to the bond fragment.

The tertiary $\mathrm{CH}$ vicinal fragment coefficients with $\mathrm{CH}$ bond fragments appear in Table 74. The observations made for the primary and secondary cases hold here too. There is now a small 'chain' effect (see Figure 2) that can be seen in the overflow fragment coefficients in the last column of Table 74 .

The coefficlents for alkyl $\mathrm{CH}$ groups vicinal to $\mathrm{CC}$ bond fragments are shown in Table 75. The overflow fragment coefficients usually appear in the first two columns, but may also occur in columns three and four (e.g. the CSCSV-H row). Their magnitudes are larger than those of the preceding 
TABLE 71. OVERFLOW ONTO PRIMARY CH FRAGMENTS IN CH LMO'S

\begin{tabular}{|c|c|c|c|c|c|c|c|}
\hline \multirow[b]{2}{*}{$\begin{array}{l}\text { CPH } \\
\text { CPH* }\end{array}$} & \multicolumn{2}{|c|}{ CPH } & \multicolumn{2}{|c|}{ CPH-H } & $T T-C P H-C$ & \multicolumn{2}{|c|}{$\mathrm{CPH}-\mathrm{C}$} \\
\hline & $\begin{array}{r}-460 \\
370\end{array}$ & $\begin{array}{r}440 \\
-345\end{array}$ & $\begin{array}{r}170 \\
-245\end{array}$ & $\begin{array}{r}-160 \\
240\end{array}$ & & & \\
\hline $\begin{array}{l}\mathrm{CSH}-\mathrm{H} \\
\mathrm{CSH}-\mathrm{C} \\
\mathrm{CSH}-2 \\
\mathrm{CSH}-3 \\
\mathrm{CSH}-2 *\end{array}$ & $\begin{array}{r}-460 \\
-465 \\
-465 \\
-455 \\
-455\end{array}$ & $\begin{array}{l}440 \\
440 \\
445 \\
440 \\
430\end{array}$ & $\begin{array}{l}165 \\
165 \\
165 \\
160 \\
165\end{array}$ & $\begin{array}{l}-150 \\
-150 \\
-150 \\
-150 \\
-155\end{array}$ & $\begin{array}{l}175-160 \\
175-160 \\
180-165 \\
175-160\end{array}$ & $\begin{array}{l}190 \\
185\end{array}$ & $\begin{array}{l}-180 \\
-175\end{array}$ \\
\hline $\begin{array}{l}\text { CTH-HH } \\
\text { CTH-H2 } \\
\text { CTH-H3 } \\
\text { CTH-23 }\end{array}$ & $\begin{array}{l}-465 \\
-465 \\
-460 \\
-460\end{array}$ & $\begin{array}{l}440 \\
440 \\
445 \\
450\end{array}$ & & & $\begin{array}{l}175-160 \\
175-160 \\
170-160\end{array}$ & $\begin{array}{l}185 \\
180 \\
180\end{array}$ & $\begin{array}{l}-175 \\
-170 \\
-170\end{array}$ \\
\hline$\cdots$ & & & 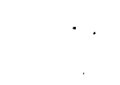 & & $\mathrm{CPH}-2$ & & \\
\hline $\begin{array}{l}\text { CSVH-H } \\
\text { CSVH }\end{array}$ & $\begin{array}{r}-415 \\
315\end{array}$ & $\begin{array}{r}410 \\
-295\end{array}$ & & & $\begin{array}{rr}140 & -130 \\
-225 & 225\end{array}$ & & \\
\hline
\end{tabular}




\begin{tabular}{|c|c|c|c|c|c|c|}
\hline & $\mathrm{CPH}$ & $\mathrm{CSH}$ & $\begin{array}{l}\mathrm{CSH} \\
2\end{array}$ & $\begin{array}{l}\mathrm{CSH} \\
3\end{array}$ & CTH & CSVH \\
\hline $\begin{array}{l}\text { CS } \\
H-H H\end{array}$ & $\begin{array}{r}-440 \\
425\end{array}$ & $\begin{array}{r}-440 \\
425\end{array}$ & $\begin{array}{r}-445 \\
425\end{array}$ & $\begin{array}{r}-435 \\
430\end{array}$ & $\begin{array}{r}-445 \\
425\end{array}$ & $\begin{array}{r}-400 \\
395\end{array}$ \\
\hline $\begin{array}{l}\mathrm{CS} \\
\mathrm{H}-\mathrm{HH}\end{array}$ & $\begin{array}{r}180 \\
-150\end{array}$ & $\begin{array}{r}175 \\
-145\end{array}$ & $\begin{array}{r}175 \\
-140\end{array}$ & $\begin{array}{r}165 \\
-140\end{array}$ & & \\
\hline $\begin{array}{l}\mathrm{CS} \\
\mathrm{H}-\mathrm{HC}\end{array}$ & & & & & $\begin{array}{r}180 \\
-150\end{array}$ & \\
\hline $\begin{array}{l}\mathrm{CS} \\
\mathrm{H}-\mathrm{H} 2\end{array}$ & $\begin{array}{r}180 \\
-150\end{array}$ & & & & & $\begin{array}{r}145 \\
-125\end{array}$ \\
\hline $\begin{array}{l}C S \\
C P-H\end{array}$ & $\begin{array}{r}105 \\
-105\end{array}$ & & & & & \\
\hline $\begin{array}{l}C S \\
C P-C\end{array}$ & $\begin{array}{r}105 \\
-105\end{array}$ & $\begin{array}{r}110 \\
-110\end{array}$ & $\begin{array}{r}105 \\
-105\end{array}$ & $\begin{array}{r}110 \\
-115\end{array}$ & $\begin{array}{r}105 \\
-105\end{array}$ & \\
\hline $\begin{array}{l}C S \\
C P-2\end{array}$ & & & & & & $\begin{array}{r}85 \\
-85\end{array}$ \\
\hline $\begin{array}{l}\text { CS } \\
\text { CS }-H H \\
\text { CS } \\
\text { CS-HC } \\
\text { CS } \\
\text { CS }-H 3\end{array}$ & $\begin{array}{r}105 \\
-105 \\
105 \\
-105 \\
105 \\
-105\end{array}$ & $\begin{array}{r}105 \\
-105\end{array}$ & & & & $:$ \\
\hline $\begin{array}{l}\text { CS } \\
\text { CT-HHH }\end{array}$ & $\begin{array}{r}105 \\
-105\end{array}$ & & & & & \\
\hline $\begin{array}{l}\text { CS } \\
\text { CSV }-H H\end{array}$ & $\begin{array}{r}100 \\
-105\end{array}$ & & & & & \\
\hline
\end{tabular}




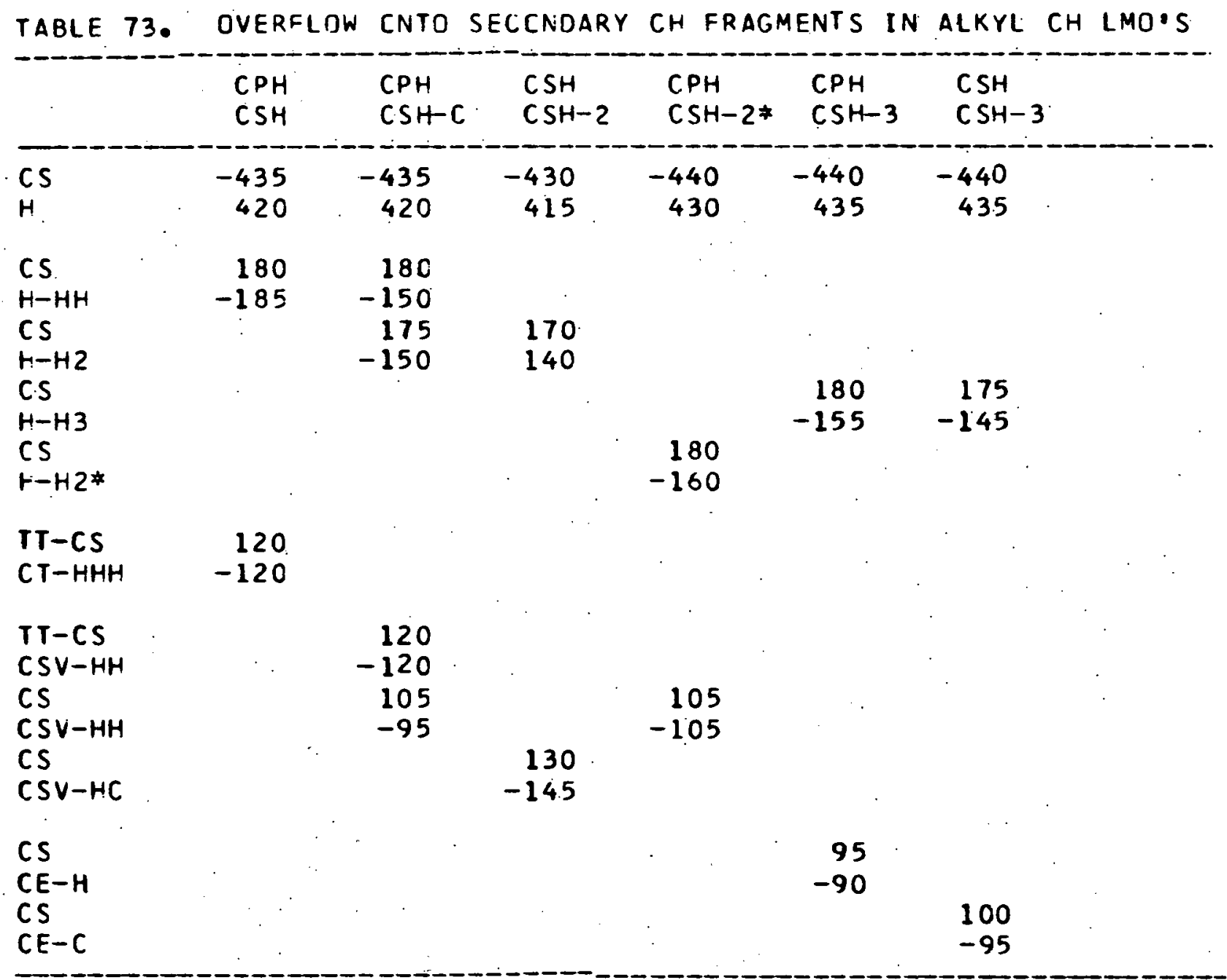


TABLE 74. OVERFLOW ONTO TERTIARY CH. FRAGMENTS IN ALKYL CH LYO'S

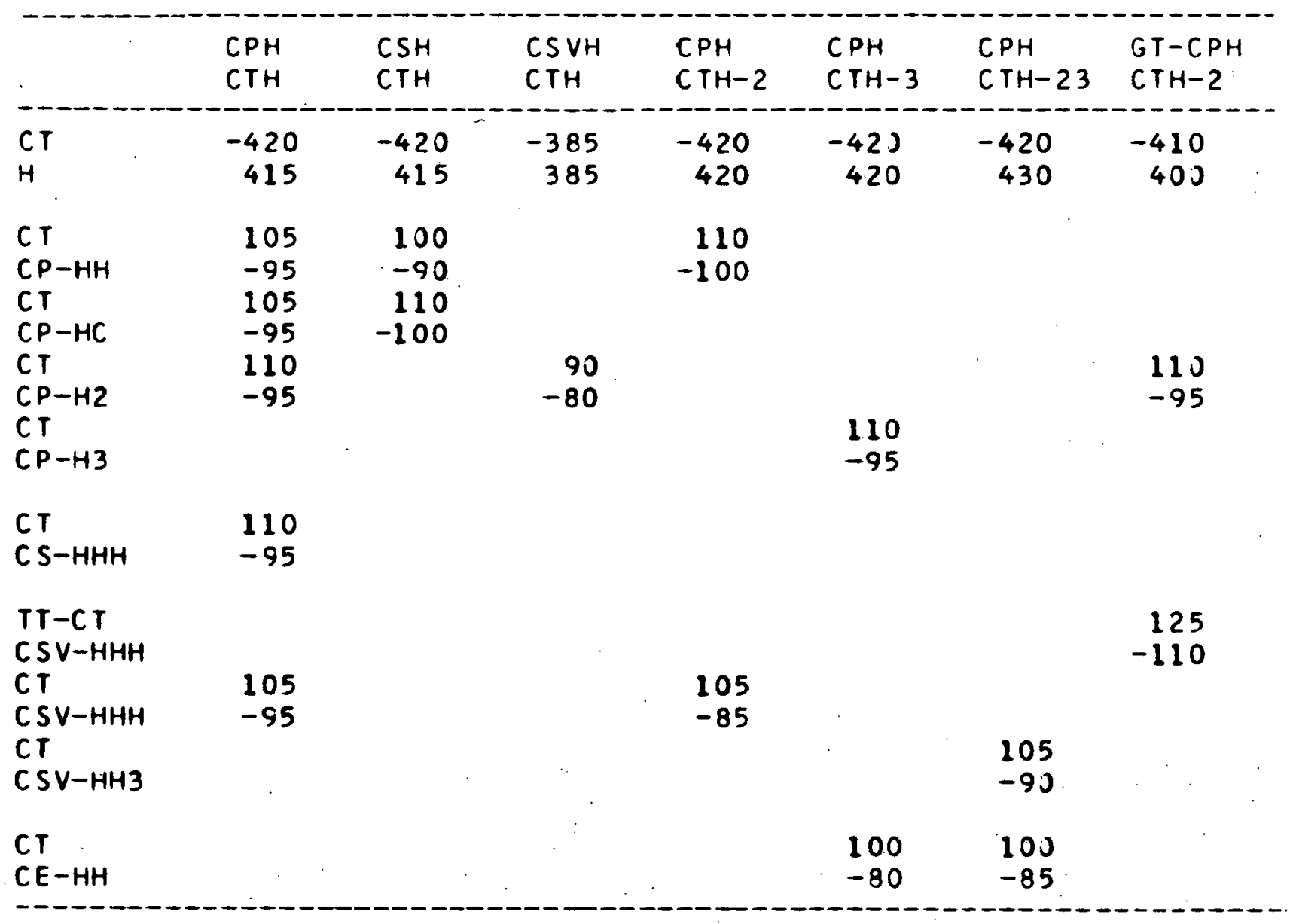


TABLE 75. OVERFLOW CNTO PRIMARY AND SECONDARY CH FRAGMENTS IN CC LMO'S

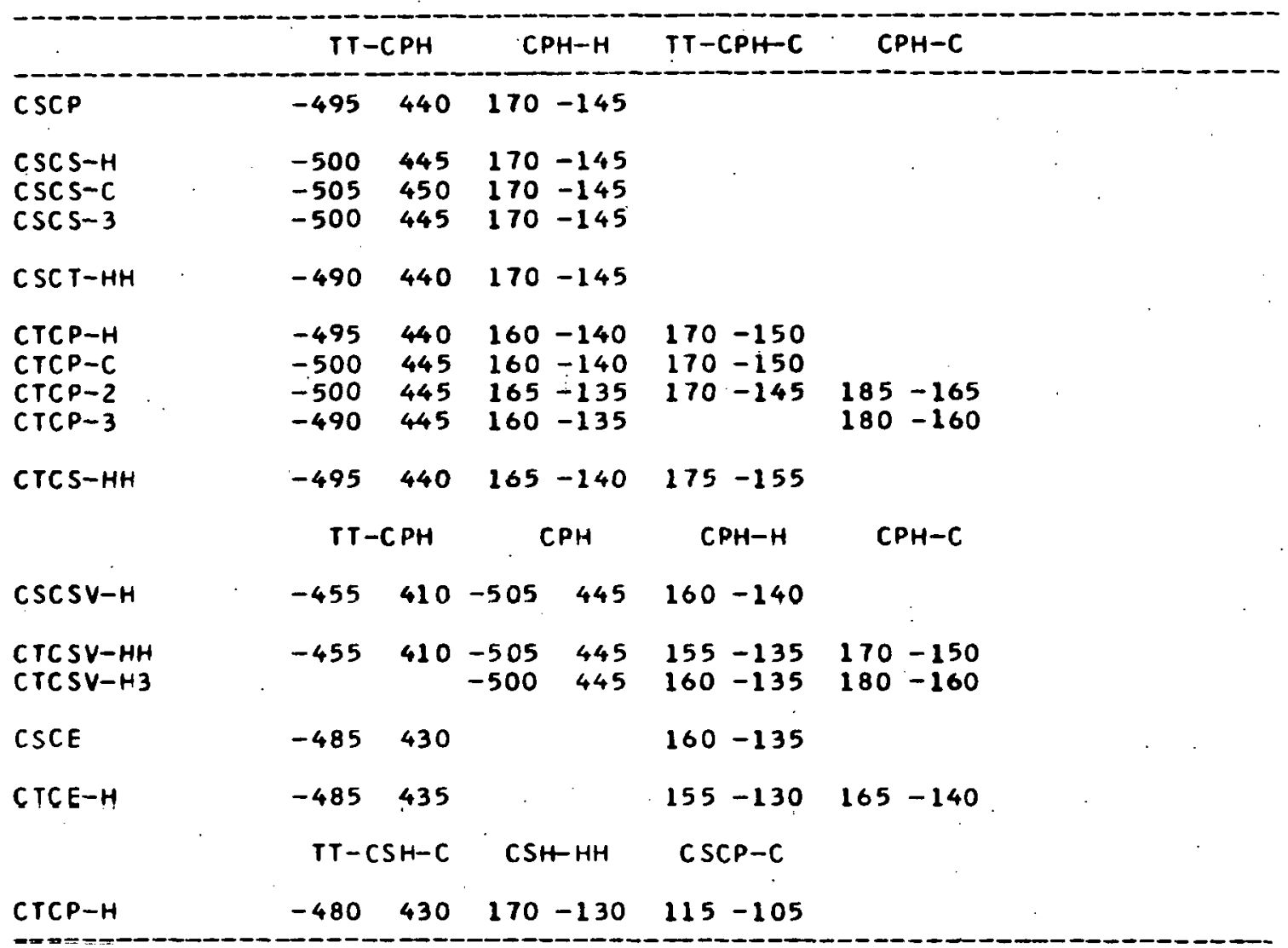


section, but those of the gauche fragments are little changed. The small coefficient variations among all fragments can be understood as in the previous section.

The coefficients for primary $\mathrm{CH}$ overflow fragments that are vicinal to double bond fragments appear in the first two columns of Table 76. Those of the secondary and tertiary overflow fragments are shown in the first two rows of Table 77. All have slightly larger magnitudes than their counter parts vicinal to $C C$ bond fragments. The gauche fragment coefficient magnitudes are, however, substantially larger than those vicinal to $C C$ bond fragments. The slightly larger coefficient variations found for ali of these vicinal fragments are due to the same factors discussed previously.

Vicinal $\mathrm{CH}$ group coefficients with triple bond fragments are shown in Table 78. Their overflow fragment coefficients are like those vicinal to $\mathrm{CH}$ bond fragments. However, their gauche fragment coefficients are like those vicinal to double bond fragments. All coefficient variations arise for reasons similar to those previously given.

\section{Alkyl CC groups}

As a whole, all alkyl CC group coefficients are smaller than those of corresponding alkyl $\mathrm{CH}$ group members. This is particularly evident for the overflow fragments. However, coefficients for a particular vicinal fragment in each of the four bond fragment classes $(\mathrm{CH}, \mathrm{CC}$, double and triple banana) stand in about the same relationship as corresponding fragment coefficients in the alkyl $\mathrm{CH}$ groups. Similarly, small coefficient 
TABLE 76. OVERFLOW CNTO PRIMARY CH FRAGMENTS IN DOUBLE BANANA LMO'S

\begin{tabular}{|c|c|c|c|c|c|c|}
\hline \multirow[b]{2}{*}{ BCSVCPV } & \multicolumn{2}{|c|}{ CPH } & \multicolumn{2}{|c|}{$\mathrm{CPH}-\mathrm{H}$} & \multirow[t]{2}{*}{$\mathrm{CPH} C$} & $\mathrm{CPH}-2$ \\
\hline & -510 & 490 & 190 & -170 & & $190-190$ \\
\hline eCSVCSV $-H$ & -525 & 495 & 195 & -170 & & $200-190$ \\
\hline $\begin{array}{l}\text { BCTVCPV-H } \\
\text { ECTVCPV-2 }\end{array}$ & $\begin{array}{l}-505 \\
-505\end{array}$ & $\begin{array}{l}485 \\
485\end{array}$ & & & $\begin{array}{l}195-175 \\
195-175\end{array}$ & $\begin{array}{l}180-180 \\
175-175\end{array}$ \\
\hline BCSVCPV* & 415 & -395 & -280 & 260 & · & -260 \\
\hline
\end{tabular}




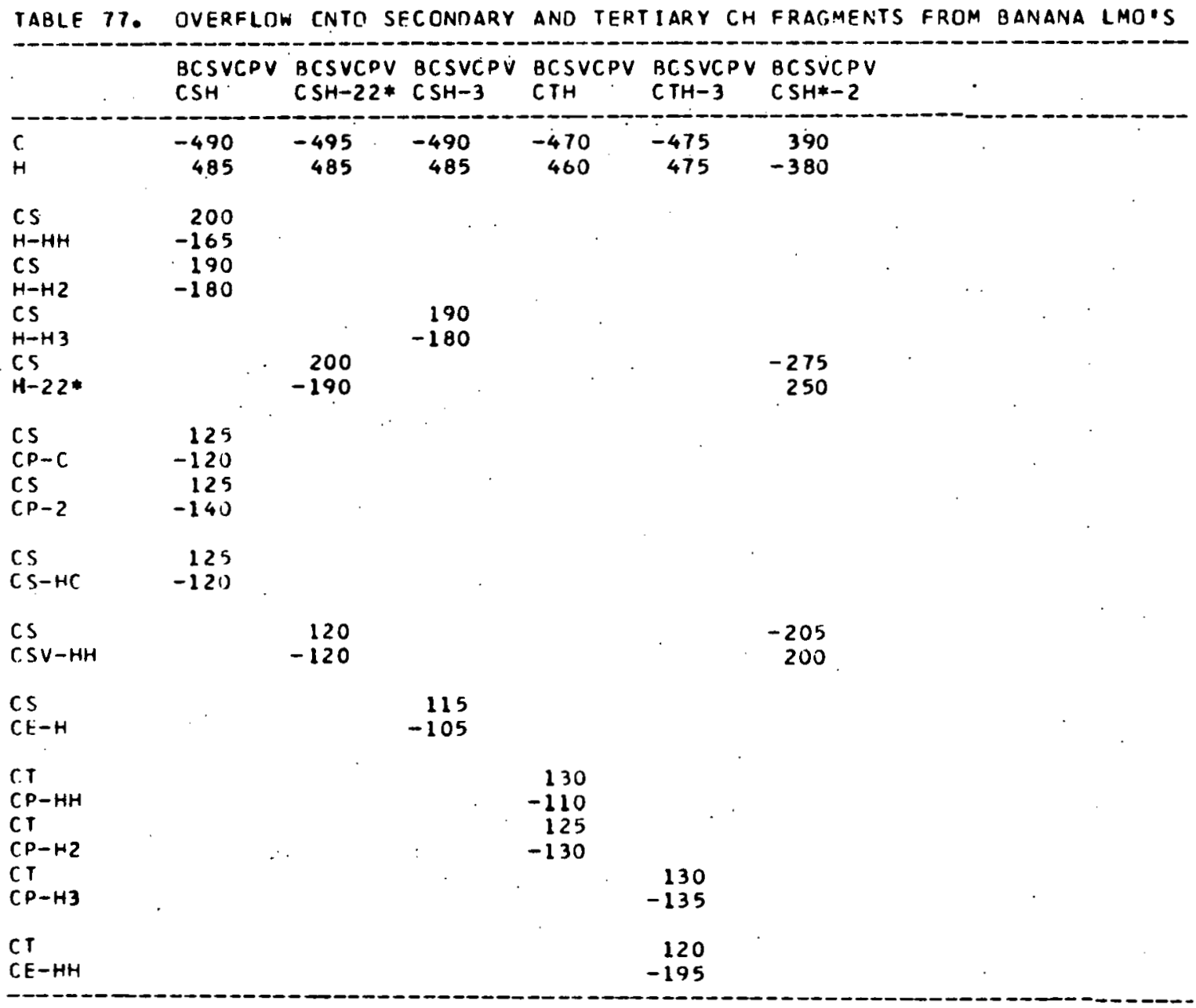


TABLE 78. OVERFLOW ONTO CH FRAGMENTS IN TRIPLE BANANA LMO'S

\begin{tabular}{|c|c|c|c|c|c|c|c|c|}
\hline & $\begin{array}{l}\text { BCSECSE } \\
\text { CPH }\end{array}$ & $\begin{array}{l}\text { BCSECPE } \\
\text { CSH }\end{array}$ & $\begin{array}{l}\text { BC SECPE } \\
\text { CSH-2 }\end{array}$ & $\begin{array}{l}\text { BCSECPE } \\
\text { CSH-3 }\end{array}$ & $\begin{array}{l}\text { BCSECPE } \\
\text { CTH }\end{array}$ & $\begin{array}{l}\text { BCSECPE } \\
\text { CTH-2 }\end{array}$ & $\begin{array}{l}\text { BCSECPE } \\
\text { CPH* }\end{array}$ & $\begin{array}{l}\text { QCSECPE } \\
\text { CSVH }\end{array}$ \\
\hline $\begin{array}{l}C \\
H\end{array}$ & $\begin{array}{r}-480 \\
495\end{array}$ & $\begin{array}{r}-465 \\
490\end{array}$ & $\begin{array}{r}-455 \\
480\end{array}$ & $\begin{array}{r}-465 \\
495\end{array}$ & $\begin{array}{r}-450 \\
480\end{array}$ & $\begin{array}{r}-440 \\
470\end{array}$ & $\begin{array}{r}420 \\
-425\end{array}$ & $\begin{array}{r}325 \\
-360\end{array}$ \\
\hline $\begin{array}{l}C P \\
\mathrm{H}-3\end{array}$ & $\begin{array}{r}190 \\
-160\end{array}$ & . & & & & & $\begin{array}{r}-255 \\
363\end{array}$ & \\
\hline . & & & & & & & & \\
\hline $\begin{array}{l}\mathrm{CS} \\
\mathrm{H}-\mathrm{H}_{3}\end{array}$ & & $\begin{array}{r}205 \\
-180\end{array}$ & & . & & & & \\
\hline $\begin{array}{l}\mathrm{CS} \\
\mathrm{H}-23\end{array}$ & & & $\begin{array}{r}205 \\
-180\end{array}$ & & & & . & \\
\hline $\begin{array}{l}C S \\
H-33\end{array}$ & & & & $\begin{array}{r}205 \\
-180\end{array}$ & & & & \\
\hline $\begin{array}{l}\mathrm{CS} \\
\mathrm{CP}-3\end{array}$ & . & $\begin{array}{r}125 \\
-140\end{array}$ & & & . & & & ' \\
\hline $\begin{array}{l}\mathrm{CS} \\
\mathrm{CS}-\mathrm{H}_{3}\end{array}$ & & $\begin{array}{r}125 \\
-140\end{array}$ & & & & . & & \\
\hline $\begin{array}{l}\mathrm{CS} \\
\mathrm{CSV}-\mathrm{H}_{3}\end{array}$ & & & $\begin{array}{r}120 \\
-120\end{array}$ & & & & & \\
\hline $\begin{array}{l}C S \\
C E-3\end{array}$ & & & & $\begin{array}{r}115 \\
-105\end{array}$ & & & & \\
\hline $\begin{array}{l}\mathrm{CT} \\
\mathrm{r} . \mathrm{P}-\mathrm{H}_{3}\end{array}$ & & & & & $\begin{array}{r}135 \\
-115\end{array}$ & & & \\
\hline $\begin{array}{l}C T \\
C P-23\end{array}$ & & & & & & $\begin{array}{r}135 \\
-135\end{array}$ & & \\
\hline $\begin{array}{l}C T \\
C S V-23\end{array}$ & $\ddots$ & & & & & $\begin{array}{r}120 \\
-140\end{array}$ & & . \\
\hline $\begin{array}{l}\text { BCSV } \\
\text { CPV } *-3 *\end{array}$ & & & r & & & & & $\begin{array}{r}-190 \\
205\end{array}$ \\
\hline
\end{tabular}


variations for both the gauche and overflow fragments are induced by ( 1 ) their coplanar vicinal fragments and (2) coplanar bond chains beyond their coplanar vicinal bonds. In addition, small coefficient changes are now also induced by cother vicinal fragments coplanar with the bond fragment.

Coefficients for alkyl CC groups vicinal to $\mathrm{CH}$ bond fragments are I isted in Tables 79-82. The overflow fragments having near secondary (tertiary) carbon atoms and far alkyl carbon atoms are shown in Table 79 (Table 80). Those whose near carbon atom is secondary (tertiary) and whose far carbon atom is vinyl or ethyriyl appear in Table 81 (Table 82). The coefficients for groups vicinal to $C C$ bond fragments are given in Table 83. The TG and TC prefixes characterize configurations that are analogous to the previously encountered $T$ T and GT configurations (see Figure 2).

The alkyl CC group coefficients vicinal to double (triple) bond fragments are listed in Table 84 (Table 85).

\section{Single Bond Vinyl Groups}

Single bond vinyl groups have overflow fragments that are vinyl single bonds. The bond region between the group and its bond fragment may be either a single or a double bond. The vicinal group coefficients for the two cases can be distinguished, and thus provide two characterizations for these vinyl groups. When the bond fragment and vicinal group are double bonded, we say that there is vicinal 'delocalization through the double bond,' and when they are singly bonded, we say that there is vicinal 
154

TABLE 79. OVERFLOW CNTO SECONDARY ALKYL CC FRAGMENTS IN CH LMO'S

\begin{tabular}{|c|c|c|c|c|c|c|c|c|}
\hline & $\begin{array}{l}\text { CPH } \\
\text { CSCP }\end{array}$ & $\begin{array}{l}\text { CPH } \\
\text { CSCS }\end{array}$ & $\begin{array}{l}\mathrm{CPH} \\
\mathrm{CSCS}-\mathrm{C}\end{array}$ & $\begin{array}{l}\mathrm{CPH} \\
\mathrm{CSCS}-3\end{array}$ & $\begin{array}{l}\text { CPH } \\
\text { CSCT }\end{array}$ & $\begin{array}{l}\text { CSVH } \\
\text { CSCP }\end{array}$ & $\begin{array}{l}\text { CSVH } \\
\text { CSCS }\end{array}$ & $\begin{array}{l}\text { CSVH } \\
\text { CSCP* }\end{array}$ \\
\hline $\begin{array}{l}c s \\
c\end{array}$ & $\begin{array}{r}-320 \\
340\end{array}$ & $\begin{array}{r}-320 \\
345\end{array}$ & $\begin{array}{r}-320 \\
345\end{array}$ & $\begin{array}{r}-335 \\
345\end{array}$ & $\begin{array}{r}-330 \\
345\end{array}$ & $\begin{array}{r}-285 \\
320\end{array}$ & $\begin{array}{r}-290 \\
320\end{array}$ & $\begin{array}{r}195 \\
-205\end{array}$ \\
\hline $\begin{array}{l}\mathrm{CS} \\
\mathrm{H}-\mathrm{HH} \\
\mathrm{CS} \\
\mathrm{H}-\mathrm{HC} \\
\mathrm{CS} \\
\mathrm{H}-\mathrm{H} 2\end{array}$ & $\begin{array}{r}145 \\
-160\end{array}$ & $\begin{array}{r}145 \\
-155\end{array}$ & $\begin{array}{r}145 \\
-155\end{array}$ & $\begin{array}{r}145 \\
-155\end{array}$ & $\begin{array}{r}145 \\
-155 \\
145 \\
-155\end{array}$ & $\begin{array}{r}115 \\
-130\end{array}$ & $\begin{array}{r}115 \\
-130\end{array}$ & $\begin{array}{r}-200 \\
220\end{array}$ \\
\hline
\end{tabular}




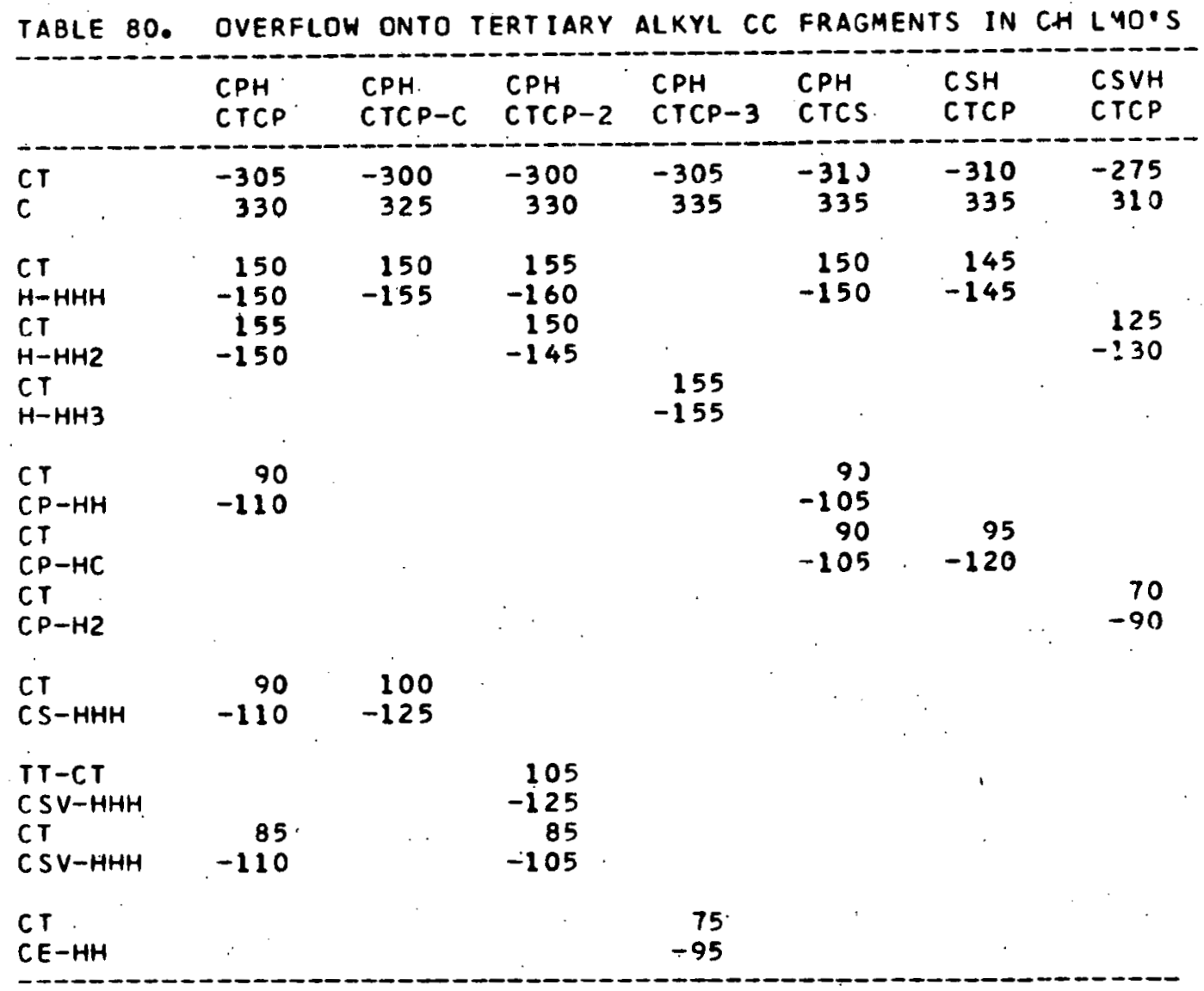


TABLE 81. OVERFLOW ONTO UNSATURATED SECONDARY ALKYL CC FRAGMENTS IN CH LMO'S

\begin{tabular}{|c|c|c|c|c|c|c|c|}
\hline & $\begin{array}{l}T T-C P H \\
\text { CSCSV }\end{array}$ & $\begin{array}{l}\text { TG-CPH } \\
\text { CSCSV }\end{array}$ & $\begin{array}{l}\text { TC }-C P H \\
\text { CSCSV }\end{array}$ & $\begin{array}{l}\text { CPH } \\
\text { CSCE }\end{array}$ & $\begin{array}{l}\text { CSVH } \\
\text { CSCSV }\end{array}$ & $\begin{array}{l}\text { CSVH } \\
\text { CSCE }\end{array}$ & $\begin{array}{l}\text { CSVH } \\
\text { CSCSV* }\end{array}$ \\
\hline $\begin{array}{l}\text { CS } \\
\text { C }\end{array}$ & $\begin{array}{r}-340 \\
355\end{array}$ & $\begin{array}{r}-315 \\
330\end{array}$ & $\begin{array}{r}-315 \\
335\end{array}$ & $\begin{array}{r}-305 \\
315\end{array}$ & $\begin{array}{r}-275 \\
315\end{array}$ & $\begin{array}{r}-280 \\
295\end{array}$ & $\begin{array}{r}235 \\
-245\end{array}$ \\
\hline $\begin{array}{l}\mathrm{CS} \\
\mathrm{H}-\mathrm{HH} \\
\mathrm{CS} \\
\mathrm{H}-\mathrm{H} 2\end{array}$ & $\begin{array}{r}140 \\
-155\end{array}$ & $\begin{array}{r}140 \\
-155 \\
150 \\
-175\end{array}$ & & & & & \\
\hline $\begin{array}{l}\mathrm{CS} \\
\mathrm{H}-\mathrm{H3} \\
\mathrm{CS} \\
\mathrm{H}-23\end{array}$ & & & $\begin{array}{r}145 \\
-170\end{array}$ & $\begin{array}{r}120 \\
-140\end{array}$ & & & . \\
\hline $\begin{array}{l}\mathrm{CS} \\
\mathrm{H}-\mathrm{H} 2 *\end{array}$ & & & & & $\begin{array}{r}145 \\
-155\end{array}$ & & \\
\hline $\begin{array}{l}\mathrm{CS} \\
\mathrm{H}-22 *\end{array}$ & & & & & $\begin{array}{r}120 \\
-130\end{array}$ & & $\begin{array}{r}-195 \\
215\end{array}$ \\
\hline
\end{tabular}




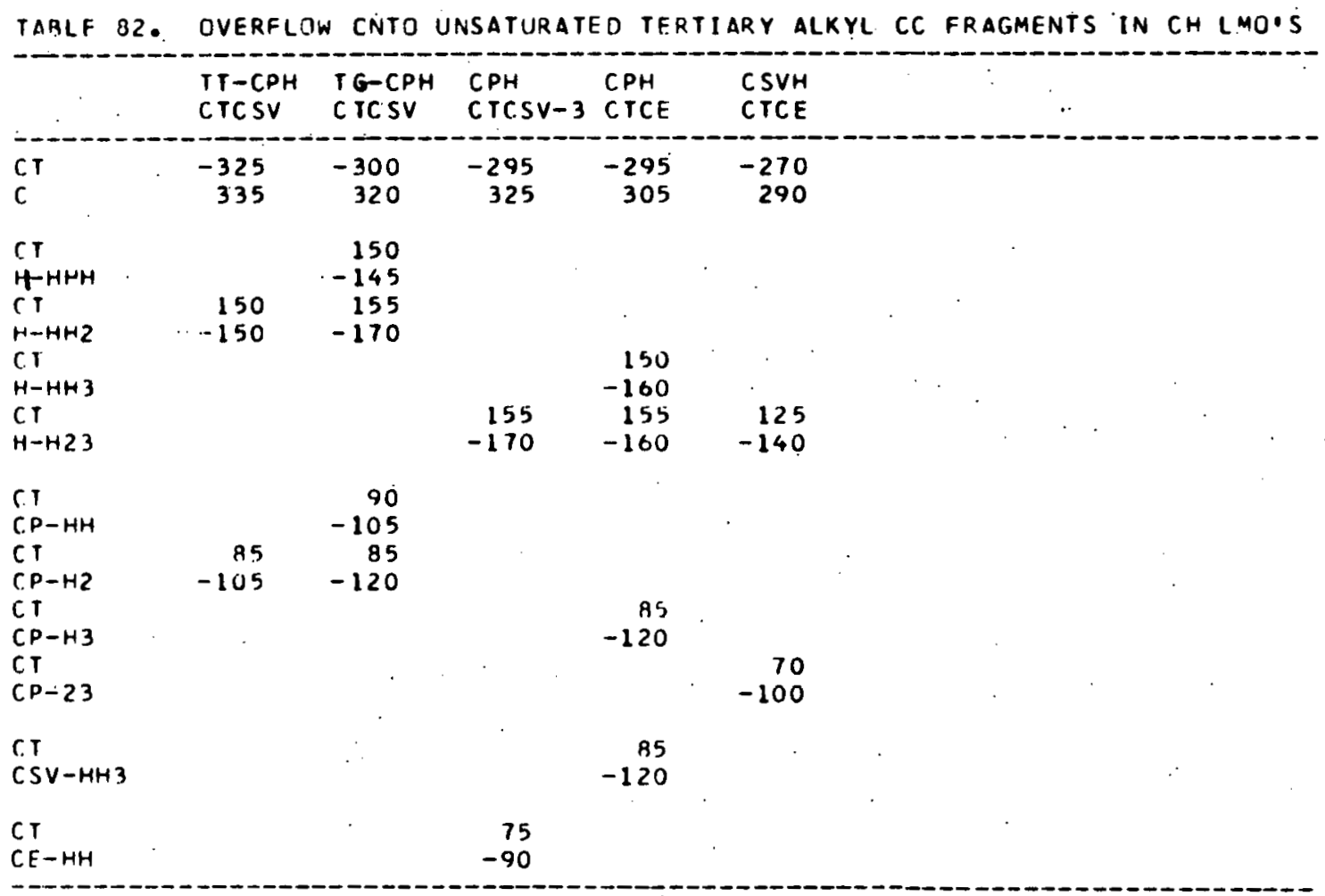


TABLE 83. OVERFLOW CNTO SECONDARY ALKYL CC FRAGMENTS IN CC LMO'S

\begin{tabular}{|c|c|c|c|c|c|c|c|c|c|}
\hline & $\begin{array}{l}\text { CSCP } \\
\operatorname{CSCP}\end{array}$ & $\begin{array}{l}\text { CSCS } \\
\text { CSCP }\end{array}$ & $\begin{array}{l}\text { CTCP } \\
\text { CSCP }\end{array}$ & $\begin{array}{l}\text { CSCSV } \\
\text { CSCP }\end{array}$ & $\begin{array}{l}\text { CSCE } \\
\text { CSCP }\end{array}$ & $\begin{array}{l}\text { C SCP } \\
\text { CTCP }\end{array}$ & $\begin{array}{l}\text { CSCP } \\
\operatorname{CSCS}\end{array}$ & $\begin{array}{l}\text { CSCP } \\
\text { CSCSV }\end{array}$ & $\begin{array}{l}\text { CSCP } \\
\text { CSCE }\end{array}$ \\
\hline $\begin{array}{l}c \\
c\end{array}$ & $\begin{array}{r}-345 \\
335\end{array}$ & $\begin{array}{r}-355 \\
340\end{array}$ & $\begin{array}{r}-350 \\
335\end{array}$ & $\begin{array}{r}-315 \\
310\end{array}$ & $\begin{array}{r}-340 \\
330\end{array}$ & $\begin{array}{r}-335 \\
330\end{array}$ & $\begin{array}{r}-350 \\
340\end{array}$ & $\begin{array}{r}-365 \\
340\end{array}$ & $\begin{array}{r}-330 \\
305\end{array}$ \\
\hline $\begin{array}{l}C \\
H-H \\
C \\
H-C\end{array}$ & $\begin{array}{r}140 \\
-145\end{array}$ & $\begin{array}{r}140 \\
-145\end{array}$ & $\begin{array}{r}135 \\
-140 \\
150 \\
-145\end{array}$ & $\begin{array}{r}130 \\
-140\end{array}$ & $\begin{array}{r}130 \\
-135\end{array}$ & $\begin{array}{r}150 \\
-140\end{array}$ & $\begin{array}{r}140 \\
-145\end{array}$ & & \\
\hline $\begin{array}{l}\mathrm{C} \\
\mathrm{H}-2\end{array}$ & & & & & & .. & & $\begin{array}{r}135 \\
-140\end{array}$ & \\
\hline $\begin{array}{l}\mathrm{C} \\
\mathrm{H}-3\end{array}$ & & & & & & & & & $\begin{array}{r}140 \\
-155\end{array}$ \\
\hline $\begin{array}{l}C T \\
C P-H\end{array}$ & & & & & & $\begin{array}{r}100 \\
-110\end{array}$ & & & \\
\hline
\end{tabular}


159

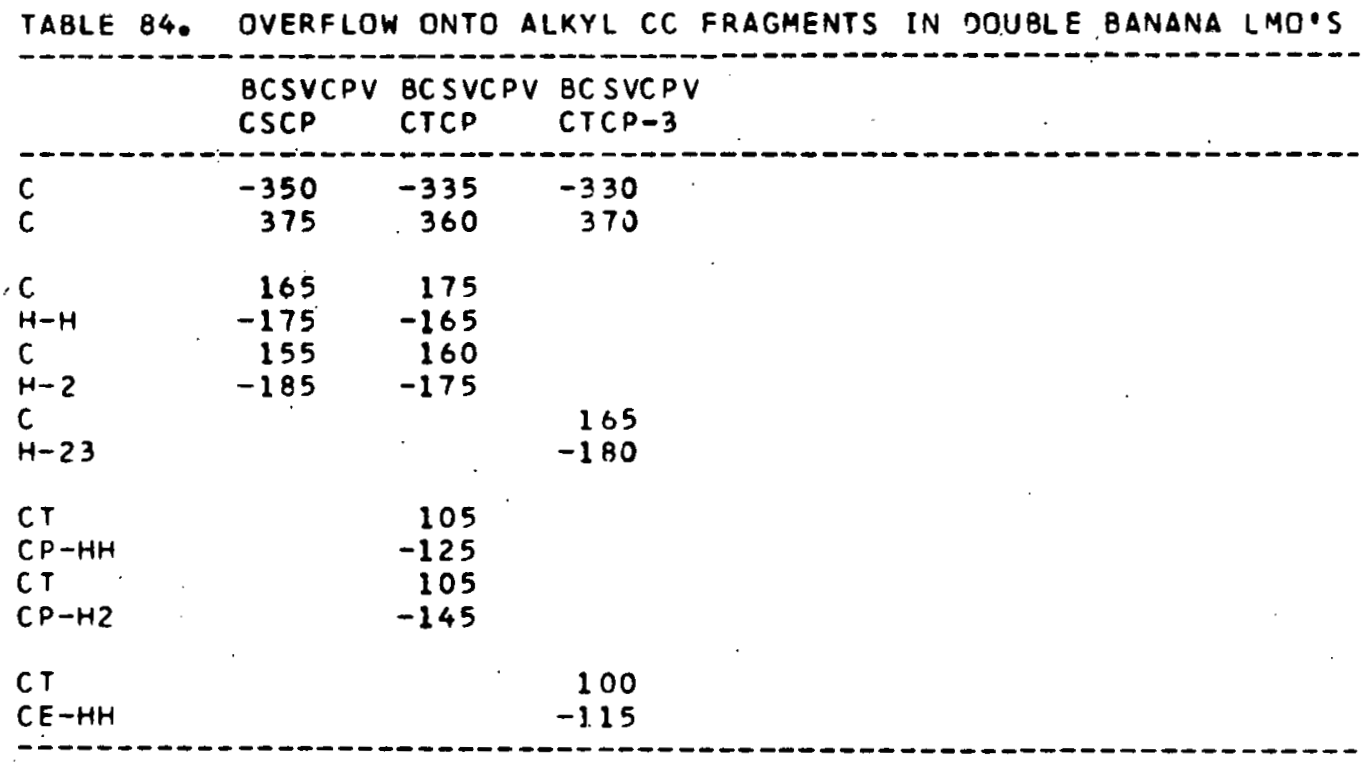




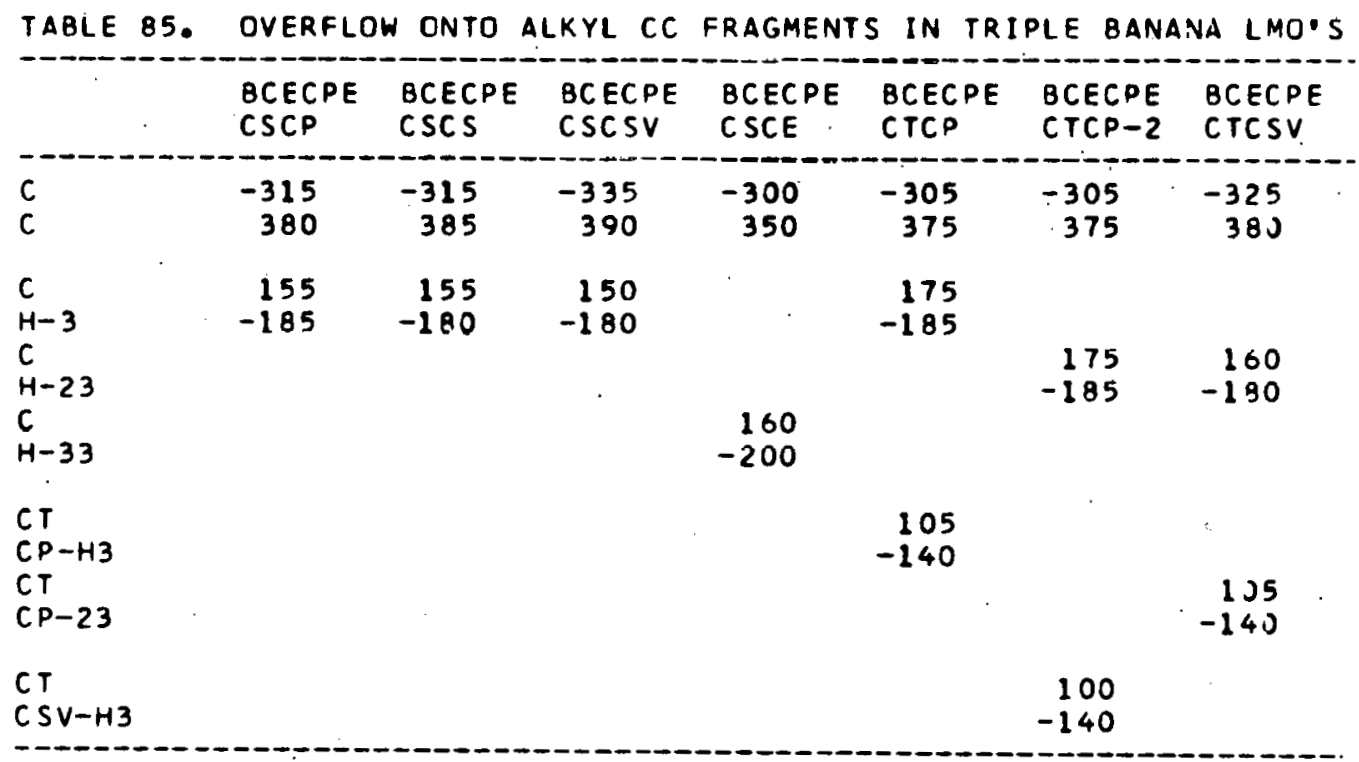


'delocalization through the single bond.' The coefficients occurring in both cases can be classified with the scheme used for the alkyl groups. Furthermore, the intra- and inter-class variations show values similar to those between the analogous alkyl group classes.

Delocalization through the double bond

When the delocalization occurs through the double bond, the vicinal group contains only two fragments. One is trans to the bond fragment, and the other is cis to the bond fragment. We shall call the trans fragment the overflow fragment. Coefficient magnitudes for both coplanar fragments are always substantially larger than those of the analogous cis or trans alkyl vicinal fragments.

The overflow fragment coefficients for delocalization through the double bond are shown in the first two columns of Table 86 for primary vinyl $\mathrm{CH}$ groups, and in the first two rows of Table 87 for secondary vinyl $\mathrm{CH}$ groups. The three letter symbols at the left end of Table 86 refer to the relative orientation of the overflow fragment and its coplanar chain beyond the bond fragment, whereas the three letter symbols in the body of Table 86, and those labelling the columns of Table 87, refer to the relative orientation of the bond fragment and its coplanar chain beyond the cis vicinal fragment. All of these were defined on the previous Figures 3 and 4. The sensitivity of the vinyl vicinal fragment coefficients to these is comparable to the sensitivity of the alkyl vicinal fragment coefficients to the TT, GT, etc. orientations.

The coefficients for the various cases of delocalization through the 
TABLE 86. IVERFLOW THRU THE DOUBLE BCND ONTO PRIMARY VINYL CH FRAGMENTS

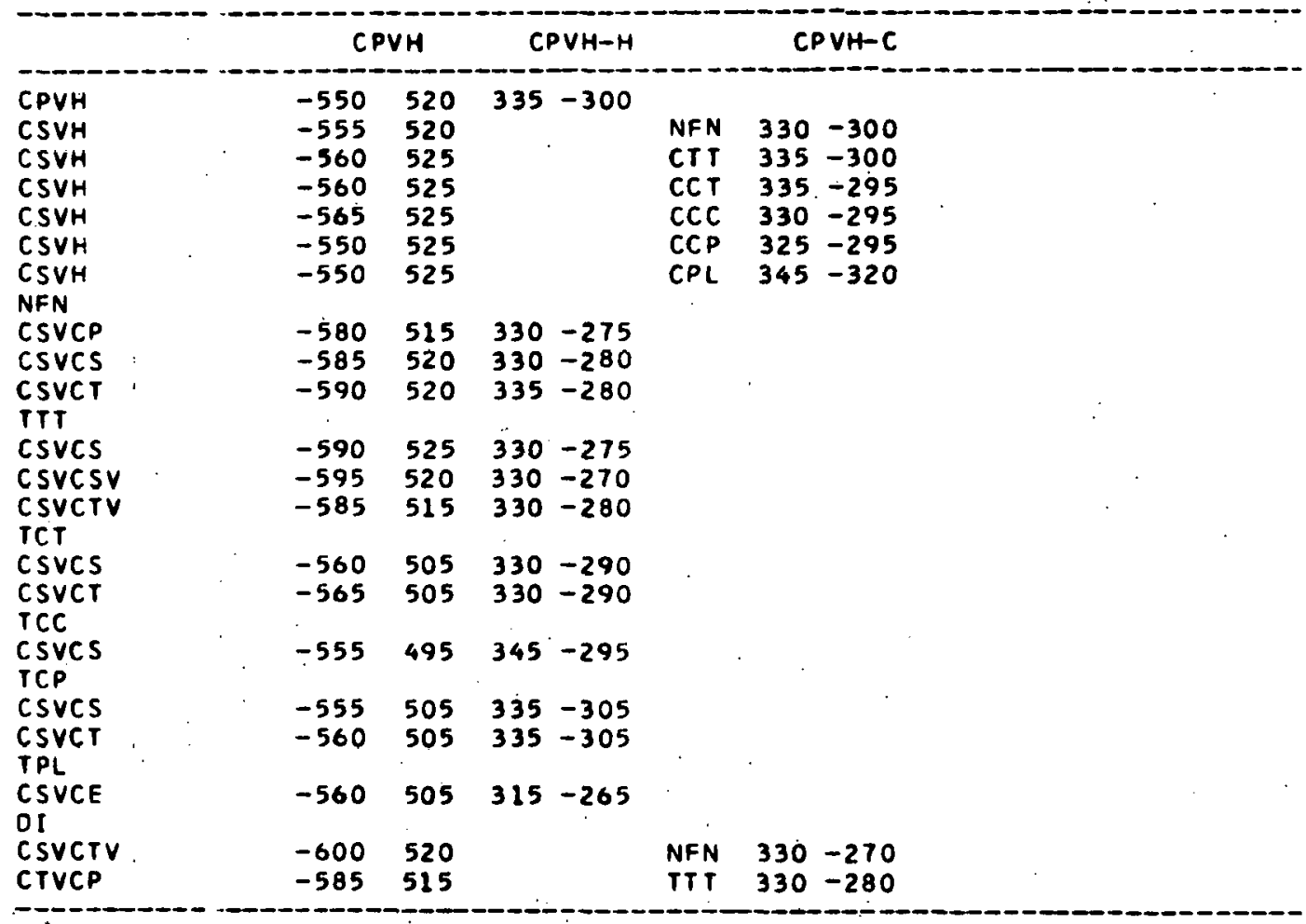


TABLE E7. DJERFLOW THROUGH THE OOUBLE BCNO ONTO SECONDARY CH FRAGMENTS IN PRIYARY ANO SECONDARY VIMYL CH LMO'S

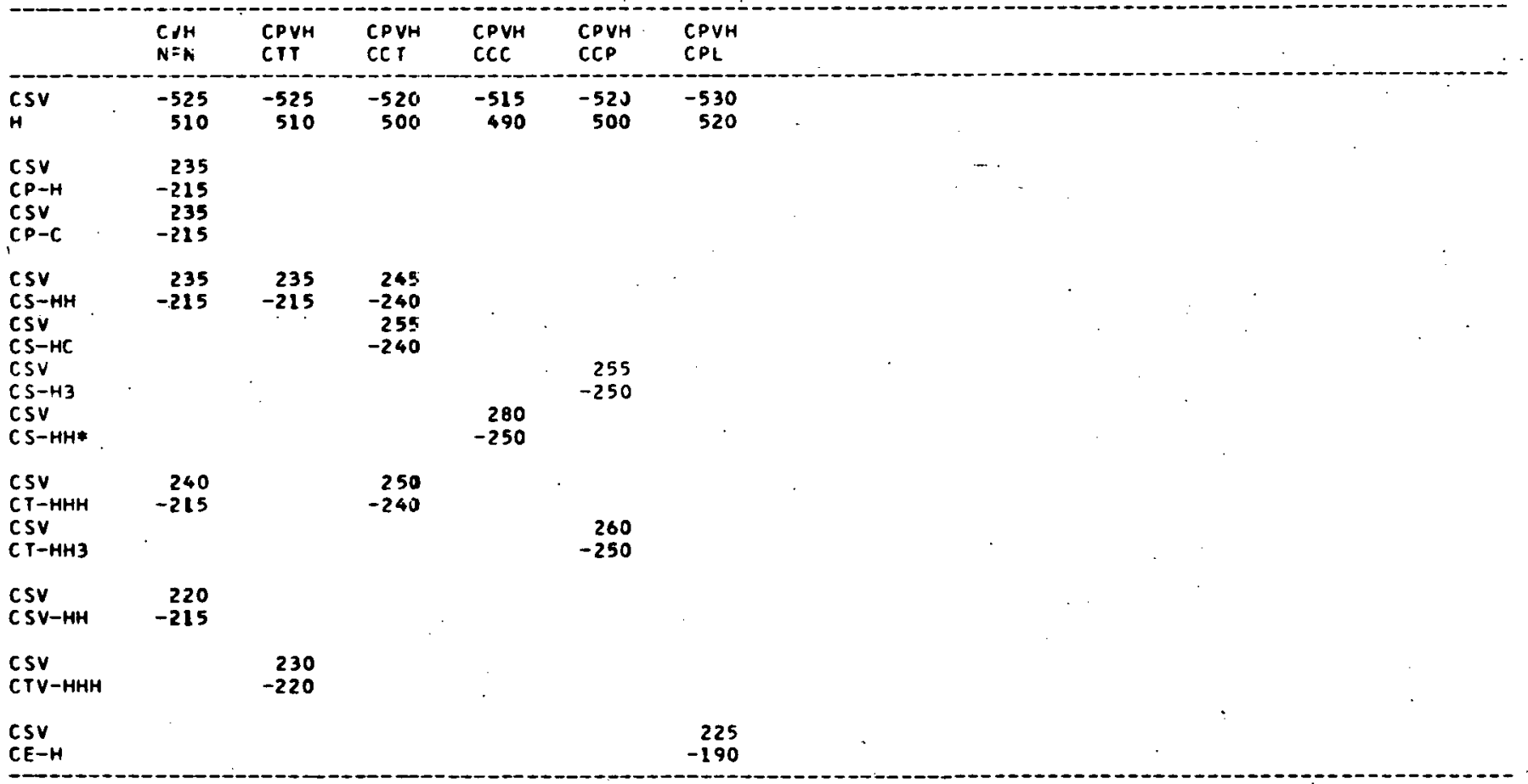


double bond onto CC groups are shown in Tables 88 and 89 . The first (second) of the column labels characterizes the bond fragment (overflow. fragment). The three letter symbols appearing as the last column labels describe the relative orientation of the bond fragment and its coplanar chains beyond the overflow fragment (see Figures 3 and 4 ).

Delocalization through the single bond

When the delocalization onto the single bond vinyl group occurs through a single bond, the vicinal group consists of a vinyl $\mathrm{CC}$ or $\mathrm{CH}$ overflow fragment and two banana gauche fragments. The overflow fragment coefficient magnitudes are nearly the same as their alkyl counterparts, but those of the banana gauche fragments are slightly larger than those of the alkyl CC gauche fragments.

The (secondary) vinyl $\mathrm{CH}$ group coefficients are listed in Tables 90 and 91 for $\mathrm{CH}$ and $\mathrm{CC}$ bond fragments, respectively. Those for the (tertiary) CC vinyl groups appear in Table 92.

Delocalization Through the Triple Bond

When the vicinal group and the bond fragment are separated by a triple bond region, the former can contain only one member. We shall call this ethynyl single bond fragment an overflow fragment since it lies along the same line as its bond fragment. Its coefficient magnitudes are about those of cis overflow fragments, with nodal properties like those of trans overfiow fragments.

The ethynyl single bond overflow fragment coefficients are listed in Table 93. The column labels characterize the bond fragment (which is 
TABLE 88, OVERFLOW THROUGH THE DOUBLE BONO ONTO VINYL UNSATURATED CC FRAGMENTS

\begin{tabular}{|c|c|c|c|c|c|c|c|c|c|c|}
\hline & $\begin{array}{l}\text { CPVH } \\
\text { CSVCP } \\
\text { NFN }\end{array}$ & $\begin{array}{l}\text { CSVCP } \\
\text { CSVCP } \\
\text { NFN }\end{array}$ & $\begin{array}{l}\text { CPVH } \\
\text { CSVCS } \\
\text { NFN }\end{array}$ & $\begin{array}{l}\text { CPVH } \\
\text { CSVES } \\
\text { TTT }\end{array}$ & $\begin{array}{l}\text { CPVH } \\
\text { CSVCS } \\
\text { TCT }\end{array}$ & $\begin{array}{l}\text { CPVH } \\
\text { CSVCS } \\
\text { TCC }\end{array}$ & $\begin{array}{l}\text { COVH } \\
\text { CSVCS } \\
\text { TCD }\end{array}$ & $\begin{array}{l}\text { CPVH } \\
\text { CSVCT } \\
\text { NFN }\end{array}$ & $\begin{array}{l}\text { CPVH } \\
\text { C SVCT } \\
\text { TCT }\end{array}$ & $\begin{array}{l}\text { CPYH } \\
\text { CSVCT } \\
\text { TCP }\end{array}$ \\
\hline $\begin{array}{l}\text { Csv } \\
\text { C }\end{array}$ & $\begin{array}{r}-415 \\
410\end{array}$ & $\begin{array}{r}-435 \\
405\end{array}$ & $\begin{array}{r}-420 \\
415\end{array}$ & $\begin{array}{r}-415 \\
415\end{array}$ & $\begin{array}{r}-425 \\
420\end{array}$ & $\begin{array}{r}-43 J \\
420\end{array}$ & $\begin{array}{r}-430 \\
425\end{array}$ & $\begin{array}{r}-425 \\
415\end{array}$ & $\begin{array}{r}-430 \\
420\end{array}$ & $\begin{array}{r}-435 \\
420\end{array}$ \\
\hline $\begin{array}{l}\text { CSV } \\
H-H H\end{array}$ & $\begin{array}{r}300 \\
-300\end{array}$ & $\begin{array}{r}300 \\
-285\end{array}$ & $\begin{array}{r}305 \\
-300\end{array}$ & & & & & $\begin{array}{r}305 \\
-300\end{array}$ & & \\
\hline $\begin{array}{l}\text { CSV } \\
H \rightarrow H C\end{array}$ & & & & & $\begin{array}{r}300 \\
-295\end{array}$ & $\begin{array}{r}300 \\
-295\end{array}$ & $\begin{array}{r}300 \\
-295\end{array}$ & & $\begin{array}{r}300 \\
-295\end{array}$ & $\begin{array}{r}30.3 \\
-295\end{array}$ \\
\hline $\begin{array}{l}\mathrm{CSV} \\
\mathrm{H}-\mathrm{HH}\end{array}$ & $\begin{array}{r}305 \\
-305\end{array}$ & & . & & & & & & & \\
\hline $\begin{array}{l}C S V \\
H-H C=\end{array}$ & & & & $\begin{array}{r}300 \\
-300\end{array}$ & & & & & & $\cdot$ \\
\hline
\end{tabular}


TABLE 89. OVERFLOW THROUGH THE DOUBLE BOND ONTO VINYL CC FRAGMENTS IN PRIMARY VINYL CH LMO'S

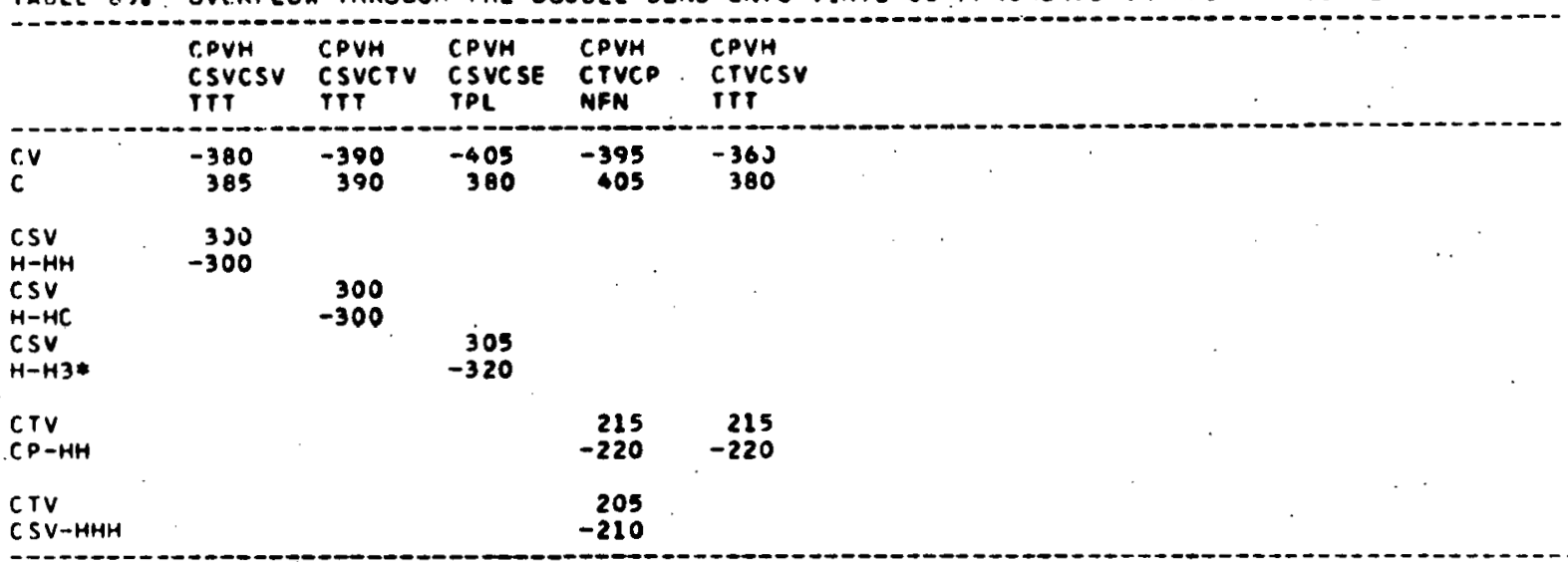


TABLE 90. OVERFLON CNTO SECENDARY VINYL CH FRAGMENTS IN CH LMO'S

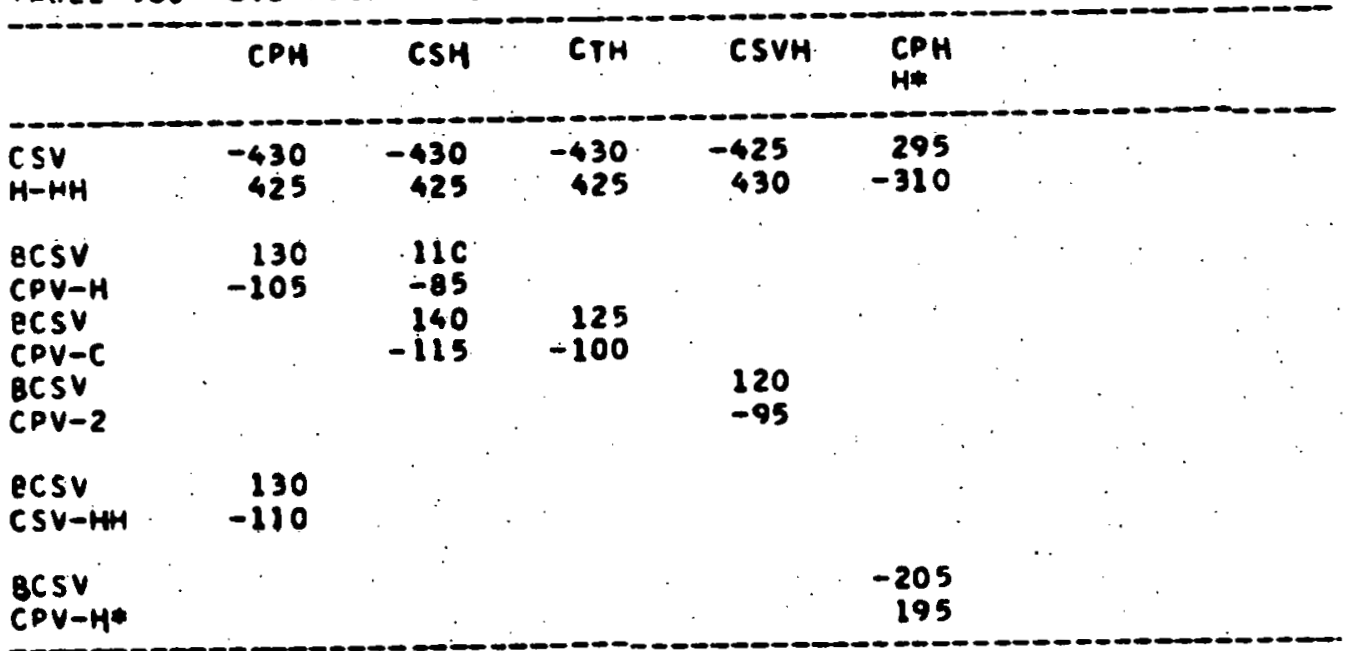




\begin{tabular}{|c|c|c|c|c|c|c|c|c|c|c|}
\hline & $i$ & $\operatorname{CSCP}$ & $\csc 5$ & CTCP & $\operatorname{cscs} v$ & CSCE & CTCE & CTUCP & $\begin{array}{l}\csc p \\
H *\end{array}$ & $\begin{array}{l}\operatorname{cscs} . \\
H\end{array}$ \\
\hline $\begin{array}{l}\text { CSV } \\
\text { H }\end{array}$ & $\vdots$ & $\begin{array}{r}-470 \\
435\end{array}$ & $\begin{array}{r}-480 \\
440\end{array}$ & $\begin{array}{r}-470 \\
435\end{array}$ & $\begin{array}{r}-485 \\
445\end{array}$ & $\begin{array}{r}-453 \\
420\end{array}$ & $\begin{array}{r}-455 \\
420\end{array}$ & $\begin{array}{r}-455 \\
435\end{array}$ & $\begin{array}{r}300 \\
-300\end{array}$ & $\begin{array}{r}325 \\
-310\end{array}$ \\
\hline $\begin{array}{l}\text { BCSV } \\
\text { CPV } \\
\text { BCSV } \\
\text { CPV-C }\end{array}$ & & $\begin{array}{r}135 \\
-100\end{array}$ & $\begin{array}{r}135 \\
-100\end{array}$ & $\begin{array}{r}120 \\
-85 \\
145 \\
-110\end{array}$ & $\begin{array}{l}130 \\
-95\end{array}$ & $\begin{array}{l}130 \\
-95\end{array}$ & $\begin{array}{r}115 \\
-80 \\
135 \\
-105\end{array}$ & & $\begin{array}{r}-220 \\
190\end{array}$ & $\begin{array}{r}-205 \\
180\end{array}$ \\
\hline $\begin{array}{l}B C T V \\
C P V-2\end{array}$ & & & : & . & & & & $\begin{array}{l}125 \\
-90\end{array}$ & & \\
\hline
\end{tabular}


TABLE 52. OVERFLOW CNTO TERTIARY VINYL CC FRAGMENTS IN CH LMO'S

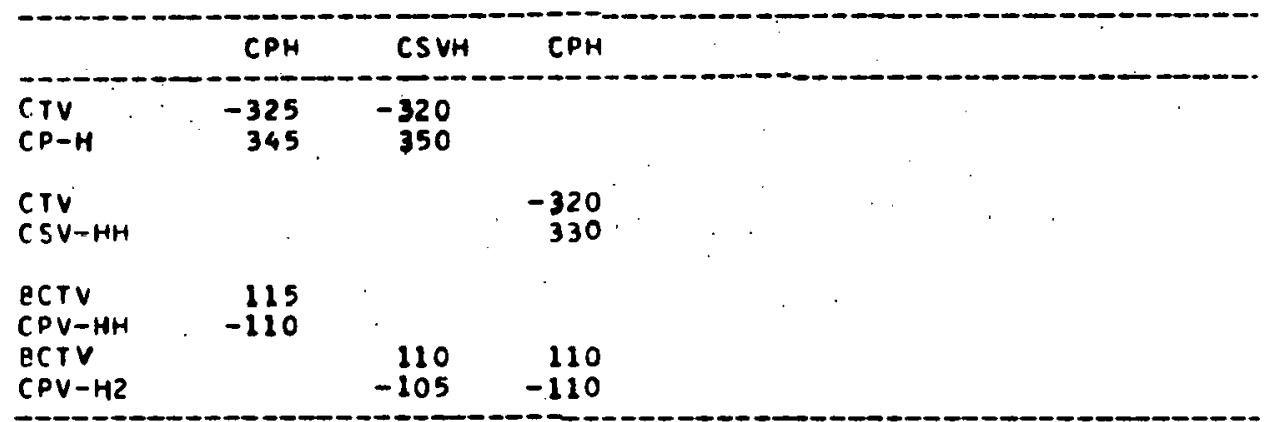




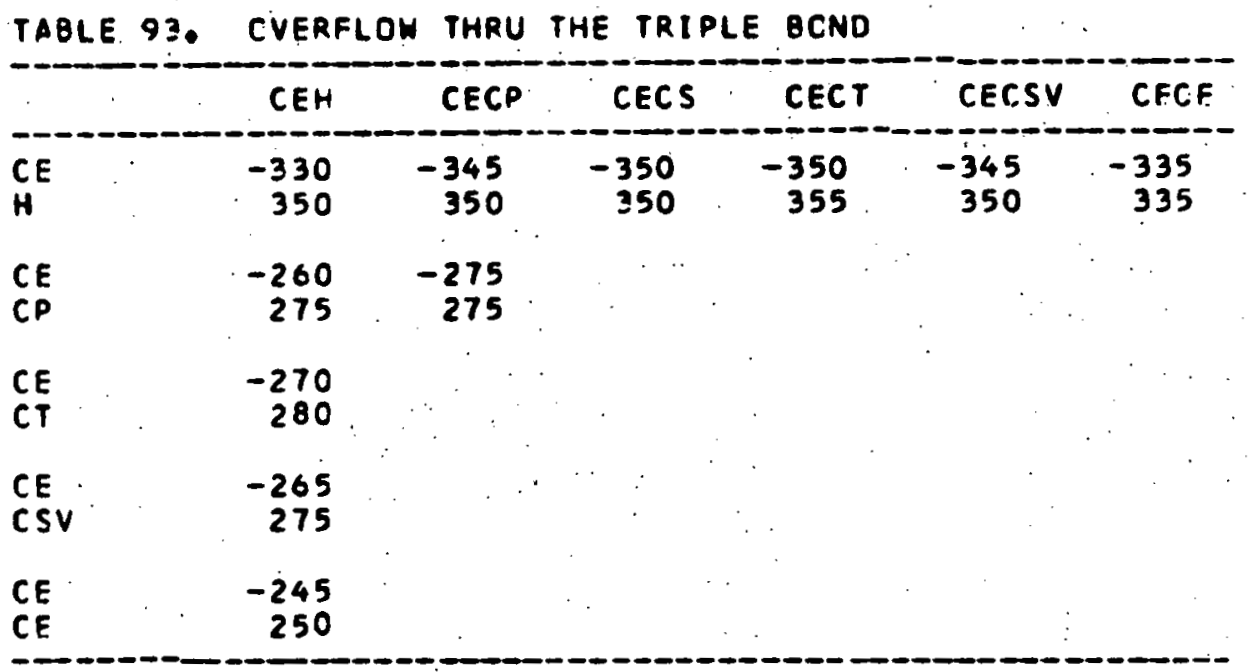




\begin{tabular}{|c|c|c|c|c|c|c|}
\hline & CEH & CECP & CECS & CECT & CECSV & CFCF \\
\hline $\begin{array}{l}C E \\
\text { CE }\end{array}$ & $\begin{array}{r}-330 \\
350\end{array}$ & $\begin{array}{r}-345 \\
350\end{array}$ & $\begin{array}{r}-350 \\
350\end{array}$ & $\begin{array}{r}-350 \\
355\end{array}$ & $\begin{array}{r}-345 \\
350\end{array}$ & $\begin{array}{r}-335 \\
335\end{array}$ \\
\hline $\begin{array}{l}C E \\
C P\end{array}$ & $\begin{array}{r}-260 \\
275\end{array}$ & $\begin{array}{r}-275 \\
275\end{array}$ & & & & \\
\hline $\begin{array}{l}\text { CE } \\
C T\end{array}$ & $\begin{array}{r}-270 \\
280\end{array}$ & & . & & & \\
\hline $\begin{array}{l}C E \\
C \subseteq V\end{array}$ & $\begin{array}{r}-265 \\
275\end{array}$ & & & & . & \\
\hline $\begin{array}{l}\text { CE } \\
\text { CE }\end{array}$ & $\begin{array}{r}-245 \\
250\end{array}$ & & & & & $\cdot$ \\
\hline
\end{tabular}


necessarily an ethynyl single bond also), and the row labels characterize the overflow fragment.

\section{Double Bond Groups}

Double bond vicinal groups are those whose overflow fragment near (banana) hybrids point into double bond regions. Their vicinal fragment coefficients can be classified with the scheme used for the single bonds and, among classes of coefficients, usually have values and variations similar to those found there.

The vicinal group coefficients having $\mathrm{CH}$ bond fragments are shown in Tables 94 and 95. The overflow fragment coefficient magnitudes are similar to those of the trans $\mathrm{CH}$ vicinal fragments observed for the delocalization through the double bond. Their other banana vicinal fragments, which lie gauche to the bond fragment, have coefficients that are larger than those of previous gauche fragments. (In fact, they are similar to the cis $\mathrm{CH}$ vicinal fragment coefficients found for the delocalization through the double bond.) However, the single bond gauche fragments have coefficient magnitudes that are slightly smaller than those observed previously.

Double bond vicinal group coefficients having C.C or banana bond fragments are given in Table 96. Those vicinal to CC or triple bond fragments are in the same relation to those vicinal to $\mathrm{CH}$ bond fragments (Tables 94 and 95) as previously observed. However, those vicinal to double bond fragments have decidedly larger magnitudes (relative to the double bond groups vicinal to $\mathrm{CH}$, $\mathrm{CC}$ or triple bond fragments). These 
TABLE 94: CVERFLOW ONTO DOU BLE BANANA FRAGMENTS IN CH LMO'S

\begin{tabular}{|c|c|c|c|c|c|c|c|c|c|}
\hline & $\begin{array}{l}\mathrm{CPH} \\
2\end{array}$ & $\begin{array}{l}\text { CSH } \\
2\end{array}$ & $\begin{array}{l}\text { CSH } \\
22 *\end{array}$ & $\begin{array}{l}\text { CSH } \\
23\end{array}$ & $\begin{array}{l}\text { CTH } \\
2\end{array}$ & $\begin{array}{l}\text { CTH } \\
23\end{array}$ & ${ }_{2 *}^{C P H}$ & $\begin{array}{l}\text { CSH } \\
2 *\end{array}$ & $\begin{array}{l}\text { CSH } \\
22 *\end{array}$ \\
\hline $\begin{array}{l}\text { BCSV } \\
\text { CPV }-H\end{array}$ & $\begin{array}{r}-560 \\
540\end{array}$ & $\begin{array}{r}-560 \\
540\end{array}$ & $\begin{array}{r}-550 \\
540\end{array}$ & $\begin{array}{r}-545 \\
540\end{array}$ & $\begin{array}{r}-560 \\
540\end{array}$ & $\begin{array}{r}-545 \\
535\end{array}$ & $\begin{array}{r}470 \\
-440\end{array}$ & $\begin{array}{r}470 \\
-440\end{array}$ & $\begin{array}{r}465 \\
-435\end{array}$ \\
\hline $\begin{array}{l}\text { CSV } \\
H+H H \\
\text { CSV } \\
H \rightarrow H C\end{array}$ & $\begin{array}{r}120 \\
-130\end{array}$ & $\begin{array}{r}110 \\
-125 \\
120 \\
-135\end{array}$ & $\begin{array}{r}125 \\
-140\end{array}$ & $\begin{array}{r}135 \\
-150\end{array}$ & $\begin{array}{r}120 \\
-135\end{array}$ & $\begin{array}{r}130 \\
-145\end{array}$ & 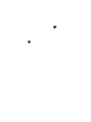 & & \\
\hline $\begin{array}{l}\text { CSV } \\
H \rightarrow H H\end{array}$ & $\therefore$ & & & & & & $\begin{array}{r}-250 \\
240\end{array}$ & $\begin{array}{r}-260 \\
250\end{array}$ & $\begin{array}{r}-260 \\
250\end{array}$ \\
\hline $\begin{array}{l}\text { CSV } \\
H \sim H C\end{array}$ & & & - & & & & & & \\
\hline $\begin{array}{l}\text { CCSV } \\
C P V-N \\
B C S V \\
C P V-C\end{array}$ & $\begin{array}{r}380 \\
-310\end{array}$ & $\begin{array}{r}325 \\
-305 \\
335 \\
-315\end{array}$ & $\begin{array}{r}315 \\
-305\end{array}$ & $\begin{array}{r}310 \\
-305\end{array}$ & $\begin{array}{r}335 \\
-315\end{array}$ & $\begin{array}{r}320 \\
-310\end{array}$ & & & . \\
\hline $\begin{array}{l}\text { eCsV } \\
\text { Cov-t. }\end{array}$ & & & & & & & $\begin{array}{r}-380 \\
375\end{array}$ & $\begin{array}{r}-360 \\
360\end{array}$ & $\begin{array}{r}-355 \\
355\end{array}$ \\
\hline
\end{tabular}


TABLE 95. CVERPLOM CNTO SECCNOARY-SECONOARY AND TERTIARY DOUBLE GANANA FRAGMENTS IN CH LMO'S

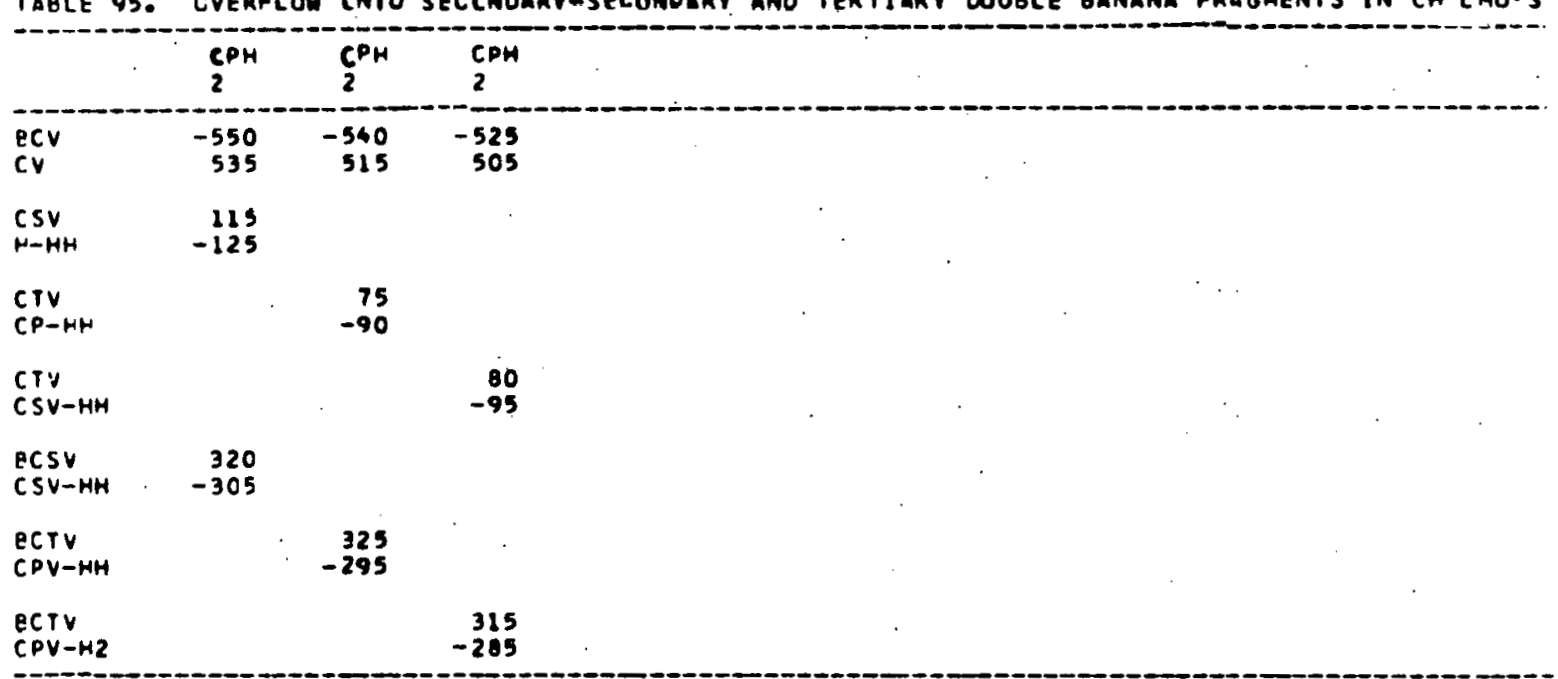


TABLE 96. OVERFLON ONTO DOUBLE BANANA FRAGMENTS IN CC AND BANANA IMO S

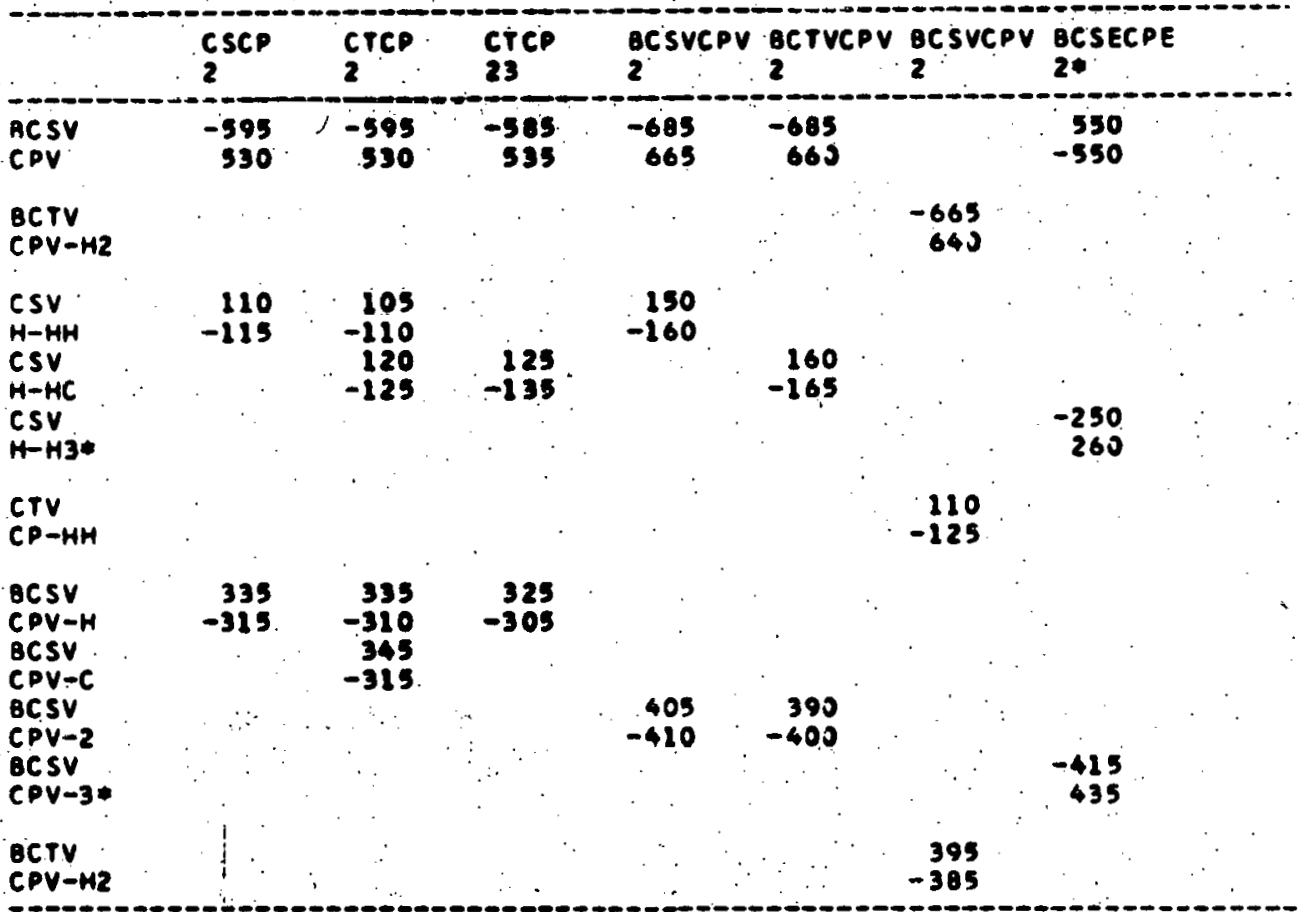


larger magnitudes are not acquired at the expense of any other particular fragment coefficients, and in fact are the only strong conjugation effects found in the present study.

\section{Triple Bond Groups}

Triple bond vicinal groups are those whose three members all have near hybrids that point into triple bond regions. Their coefficients which occur vicinal to $\mathrm{CH}$ (CC and banana) bond fragments are shown in Table. 97 (Table 98). The latter overflow fragment coefficients (Table 98) have the largest magnitudes encountered in this work. The triple bond gauche fragments have coefficient magnitudes intermediate to the double. bond and single bond gauche fragments of the preceding section. With the exception of the strong conjugation effects, which are not found here, coefficient classes and trends are similar to those of the double bond vicinal groups. 
TABLE 97. OVERFLOM CNTO TRIPLE BANANA FRAGMENTS IN CH LMO'S

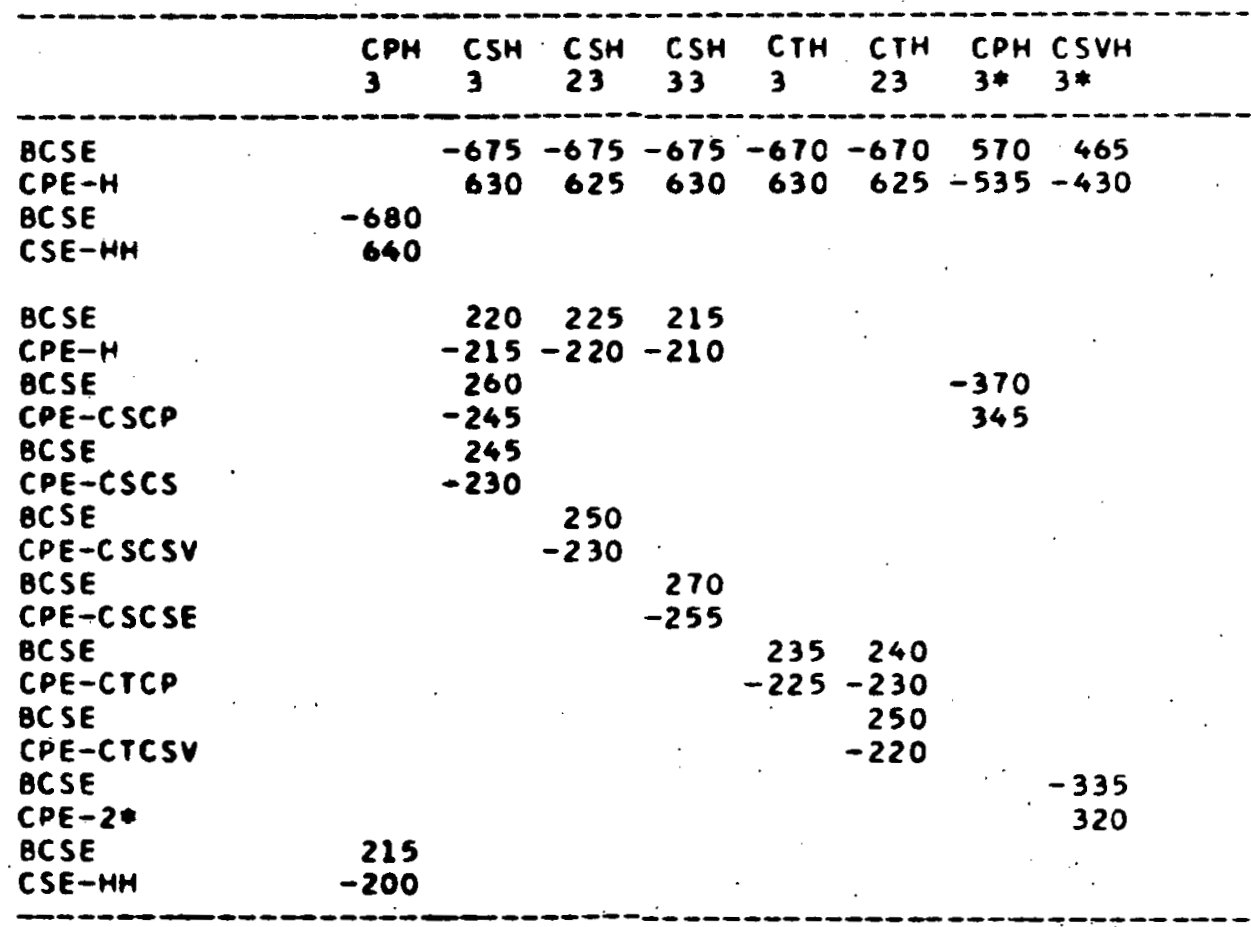


TABLE 98. OVERFLON ONTO TRIPLE BANANA FRAGMENTS IN CC ANO BANANA LMO'S

\begin{tabular}{|c|c|c|c|c|c|c|c|c|c|}
\hline & ${ }_{3}^{\csc \theta}$ & $\underset{3}{\operatorname{cscs}}$ & $\begin{array}{l}\operatorname{cscs} v \\
3\end{array}$ & $\begin{array}{l}\text { CSCE } \\
3\end{array}$ & $\begin{array}{l}\text { CTCP } \\
3\end{array}$ & $\begin{array}{l}\text { CTCP } \\
23\end{array}$ & $\begin{array}{l}\text { CTCSV } \\
3\end{array}$ & $\begin{array}{l}\text { BCECE } \\
3\end{array}$ & $\begin{array}{l}\text { 8Cver } \\
3:\end{array}$ \\
\hline $\begin{array}{l}\text { BCSE } \\
\text { CPE }\end{array}$ & $\begin{array}{r}-735 \\
630\end{array}$ & $\begin{array}{r}-745 \\
635\end{array}$ & $\begin{array}{r}-705 \\
605\end{array}$ & $\begin{array}{r}-715 \\
610\end{array}$ & $\begin{array}{r}-715 \\
625\end{array}$ & $\begin{array}{r}-715 \\
630\end{array}$ & $\begin{array}{r}-685 \\
600\end{array}$ & $\begin{array}{r}-750 \\
740\end{array}$ & $\begin{array}{r}615 \\
-595\end{array}$ \\
\hline $\begin{array}{l}\text { BCSE } \\
\text { CPE-H } \\
\text { BCSE } \\
\text { CPE-CTCP } \\
\text { BCSE } \\
\text { CPE-CTCSV } \\
\text { BCSE } \\
\text { CPE-2 }\end{array}$ & $\begin{array}{r}250 \\
-230\end{array}$ & $\begin{array}{r}250 \\
-230\end{array}$ & $\begin{array}{r}235 \\
-215\end{array}$ & $\begin{array}{r}240 \\
-220\end{array}$ & $\begin{array}{r}220 \\
-205 \\
270 \\
-245\end{array}$ & $\begin{array}{r}220 \\
-205 \\
\\
265 \\
-240\end{array}$ & $\begin{array}{r}205 \\
-190 \\
255 \\
-225\end{array}$ & & $\begin{array}{r}-420 \\
360\end{array}$ \\
\hline $\begin{array}{l}\text { BCSE } \\
\text { CPE-3 }\end{array}$ & & & & . & & & & $\begin{array}{r}250 \\
-250\end{array}$ & \\
\hline
\end{tabular}




\section{THIRD AND FOURTH NEIGHBOR FRAGMENTS}

Third Neighbors

Third neighbor fragments are those that are two bond regions removed from the bond fragment. Six types of so-called 'overfiow onto third neighbor fragments' are considered: (1) T, (2) C, (3) S, (4) S', (5) L, and (6) $P$. These symbols are defined on Figure 5 and refer to the relative orientation of the bond fragment (B) and a particular third neighbor fragment ( $F$, which is usually coplanar with the bond fragment). An asterisk following the symbol designates rotation of the fragment $F$ through $180^{\circ}$ for the type in question (e.g. T and $T *$ on Figure 5).

Distinct nodal properties, magnitudes and variations obtain for third neighbor fragment coefficients. However, partly because they are small, and partly because we have found no applications involving them, we shall content ourselves with listing them in the tables. The F fragment coefficients will always be given first, and for all third neighbor fragments we use the order (1) near (relative to the bond fragment) hybrids, (2) far hybrids.

The coefficients for $T$ and $C$ third neighbor $F$ fragments are listed in the first two columns of Tables 99 and 100 , respectively. The $\mathrm{CH}$ and $\mathrm{CC}$ column labels characterize the fragments adjacent to $F$. The row labels (e.g. $\mathrm{CH}-\mathrm{C}$ ) characterize the bond fragment ( $\mathrm{CH}$ in our example) and the $\mathrm{F}$ fragment (CC in our example), respectively. The $T *$ and $C *$ cases are denoted by the asterisk immediately following the $F$ fragment label (e.g. $\mathrm{CH}-\mathrm{C} *$ in Tables 99 and 100). 
Figure 5. Bond skeletons for third neighbor overflow 


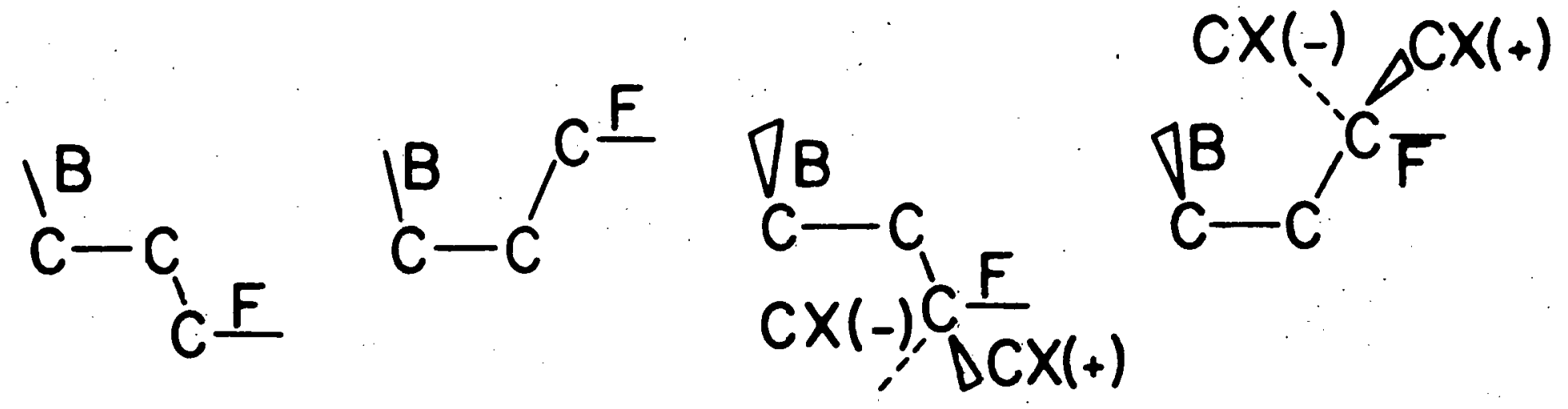

$$
\begin{aligned}
& \text { T }
\end{aligned}
$$

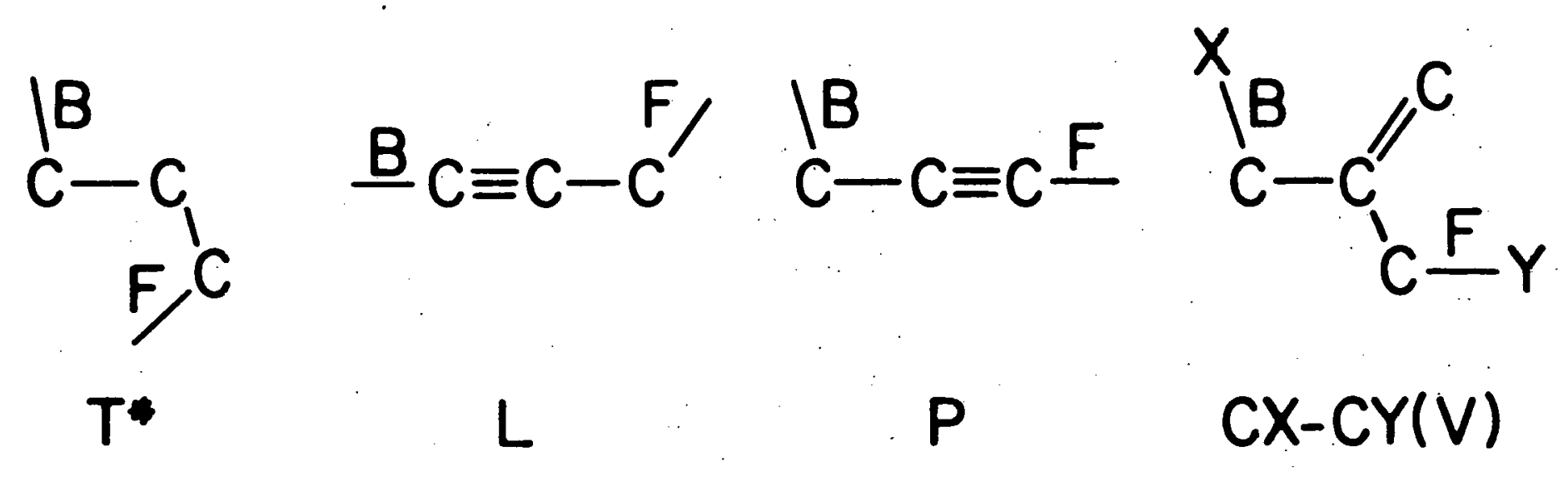


TABLE 99. T OVERFLOW ONTO THIRO NEIGHBOR FRAGMENTS

\begin{tabular}{|c|c|c|c|c|c|c|}
\hline & & $F$ & & & . & \\
\hline $\begin{array}{l}\text { CH-H } \\
\text { CSVH-H } \\
\text { CC-H } \\
\text { BCVCV-H } \\
\text { BCECE-H } \\
\text { CH-CPVH } \\
\text { CC-CPVH } \\
\text { ECECE-CPVH } \\
\text { CH-CSVH } \\
\text { CC-CSVH } \\
\text { BCECE-CSVH }\end{array}$ & $\begin{array}{l}140 \\
115 \\
145 \\
160 \\
160 \\
145 \\
150 \\
160 \\
125 \\
130 \\
135\end{array}$ & $\begin{array}{l}-135 \\
-115 \\
-130 \\
-150 \\
-150 \\
-145 \\
-140 \\
-160 \\
-135 \\
-130 \\
-140\end{array}$ & $\begin{array}{r}-15 \\
-15 \\
-20 \\
-15 \\
-15 \\
0 \\
-10 \\
5\end{array}$ & $\begin{array}{l}20 \\
15 \\
20 \\
15 \\
15 \\
25 \\
25 \\
20\end{array}$ & $\begin{array}{r}-5 \\
-10 \\
-10\end{array}$ & $\begin{array}{l}-10 \\
-10 \\
-10\end{array}$ \\
\hline $\begin{array}{l}\mathrm{CH}-\mathrm{C} \\
\mathrm{CC}-\mathrm{C} \\
\text { QCECE-C }\end{array}$ & $\begin{array}{l}100 \\
110 \\
120\end{array}$ & $\begin{array}{l}-105 \\
-105 \\
-125\end{array}$ & $\begin{array}{r}-5 \\
-10 \\
-5\end{array}$ & $\begin{array}{l}20 \\
20 \\
20\end{array}$ & 0 & 10 \\
\hline $\begin{array}{l}\mathrm{CH}-2 \\
\mathrm{CH}-3 \\
\mathrm{CC}-3 \\
\text { ECECE-3 }\end{array}$ & $\begin{array}{l}150 \\
180 \\
195 \\
210\end{array}$ & $\begin{array}{l}-165 \\
-195 \\
-195 \\
-220\end{array}$ & 10 & -5 & $\begin{array}{l}-85 \\
-30 \\
-40 \\
-35\end{array}$ & $\begin{array}{l}80 \\
25 \\
25 \\
25\end{array}$ \\
\hline $\begin{array}{l}C H-H(V) \\
C S V H-H(V) \\
C H-C S V H(V)\end{array}$ & $\begin{array}{l}100 \\
90 \\
100\end{array}$ & $\begin{array}{r}-100 \\
-90 \\
-105\end{array}$ & $\begin{array}{l}0 \\
0\end{array}$ & $\begin{array}{l}5 \\
0\end{array}$ & -5 & 10 \\
\hline $\begin{array}{l}\mathrm{CH}-\mathrm{H}^{*} \\
\mathrm{CH}-\mathrm{CSVH} \\
\mathrm{CSVH}-\mathrm{CSVH}\end{array}$ & $\begin{array}{l}-25 \\
-5 \\
-45\end{array}$ & $\begin{array}{l}45 \\
25 \\
45\end{array}$ & 80 & -80 & $\begin{array}{l}55 \\
60 \\
50\end{array}$ & $\begin{array}{l}-60 \\
-70 \\
-60\end{array}$ \\
\hline $\mathrm{CH}-\mathrm{C}$ & 5 & 25 & 70 & -75 & 50 & -60 \\
\hline
\end{tabular}


TABLE 100. C OVERFLGW ONTO THIRO NEIGHBCR FRAGMENTS

\begin{tabular}{|c|c|c|c|c|c|c|c|}
\hline \multirow[b]{2}{*}{$\begin{array}{l}\text { CH-H } \\
\text { CSVH-H } \\
\text { CH-CPVH } \\
\text { CC-CPVH } \\
\text { CSVH-CPVH } \\
\text { CSVH-CSVH }\end{array}$} & \multirow[b]{2}{*}{$\begin{array}{r}-90 \\
-140 \\
-120 \\
-125 \\
-95 \\
-55\end{array}$} & \multirow{2}{*}{$\begin{array}{l}F \\
100 \\
130 \\
125 \\
135 \\
105 \\
60\end{array}$} & \multicolumn{2}{|c|}{$\mathrm{CH}$} & \multicolumn{2}{|c|}{ CC } & \\
\hline & & & $\begin{array}{r}-10 \\
5 \\
-50 \\
-20 \\
-50\end{array}$ & $\begin{array}{r}-5 \\
-5 \\
20 \\
-10 \\
30\end{array}$ & -5 & 30 & \\
\hline $\begin{array}{l}\mathrm{CH}-\mathrm{C} \\
\mathrm{CH}-\mathrm{CS} \vee \mathrm{C}\end{array}$ & $\begin{array}{r}-80 \\
-110\end{array}$ & $\begin{array}{r}90 \\
125\end{array}$ & $\begin{array}{l}-10 \\
-50\end{array}$ & $\begin{array}{l}-5 \\
15\end{array}$ & & & \\
\hline $\mathrm{CH}-3$ & -100 & 120 & & & 10 & -15 & \\
\hline $\begin{array}{l}\mathrm{CH}-\mathrm{H}^{*} \\
\mathrm{CS} V \mathrm{H}-\mathrm{H}^{*}\end{array}$ & $\begin{array}{l}-65 \\
-60\end{array}$ & $\begin{array}{l}45 \\
35\end{array}$ & $\begin{array}{l}-45 \\
-50\end{array}$ & $\begin{array}{l}45 \\
55\end{array}$ & -40 & 45 & $\because$ \\
\hline $\begin{array}{l}\mathrm{CH}-\mathrm{C} * \\
\mathrm{CH}-\mathrm{CC} S \mathrm{~S}^{*} \\
\mathrm{CH}-\mathrm{CCE} *\end{array}$ & $\begin{array}{l}-65 \\
-55 \\
-85\end{array}$ & $\begin{array}{l}40 \\
25 \\
55\end{array}$ & $\begin{array}{l}-15 \\
-15 \\
-15\end{array}$ & $\begin{array}{l}30 \\
30 \\
30\end{array}$ & & & \\
\hline
\end{tabular}


The $S$ and $S^{\prime}$ third neighbor fragment coefficients are shown in Table 101 and the upper half of Table 102, respectively. Note that neither have F fragments coplanar with the bond fragment. The fragments $c x(+)$ and $C X(-)$ are defined in Figure, 5. The $X^{\prime} ' s$ in the tables indicate that the coefficients for the fragments in question are almost independent of type. Remaining labels have the same meaning as in Tables 99 and 100.

The remaining two third neighbor fragment coefficient categories, $L$ and $P$, are also given in Table 102. In the former, all third neighbor fragments lie on a coplanar chain with the bond fragment. In the latter, there is only one third neighbor fragment. The $X^{\prime}$ 's in (the lower half of) Table 102 stand for either $\mathrm{C}$ or $\mathrm{H}$, and the column labels characterize the third neighbor fragments.

\section{Fourth Neighbors}

Fourth neighbor fragments are three bond regions removed from the bond fragment. The nine classes are described in Figure 6: (1). TT, (2) TC, (3) $\mathrm{TP}$, (4) CT, (5) CC, (6) CP, (7) PL, (8) LP, and (9) LL. The symbols B, $F$, and the asterisk have meanings directly analogous to those used for the third neighbor fragments. Here, however, the bond fragment (B) and fourth neighbor fragment $F$ are always coplanar.

For the same reasons as were given for the third neighbor fragments, we shall content ourselves here with describing the coefficient tabulations. The $F$ fragment coefficients are given first, in the order (1) near hybrid, (2) far hybrid, and the latter order is also used for the fourth neighbor fragments adjacent to $F$. 
TABLE 101. S CVERFLCH ONTO THIRD NEIGHBCR FRAGMENTS

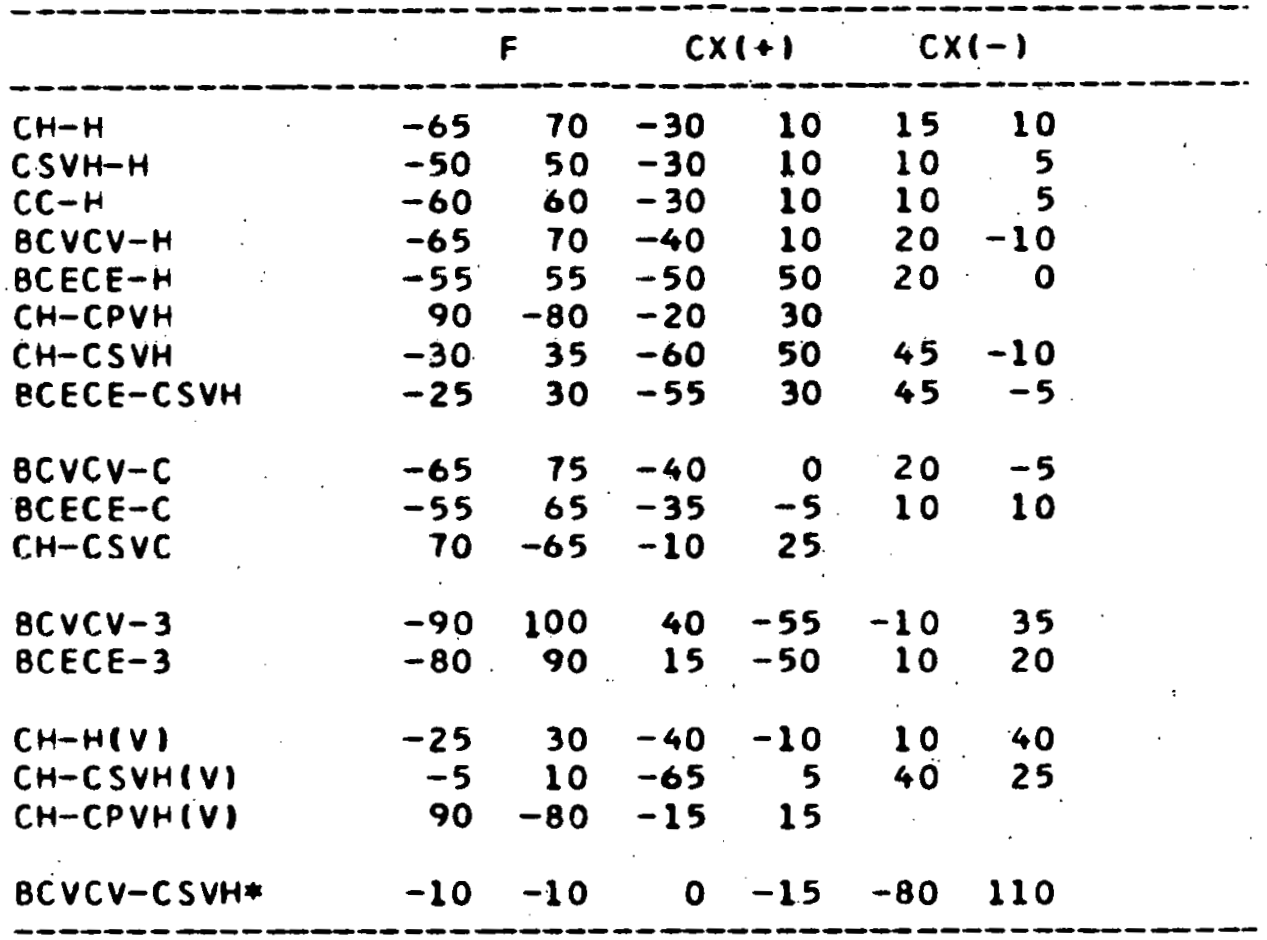


TABLE 102. 'S', L AND P OVERFLOW ONTO THIRD NEIGHBOR FRAGMENTS

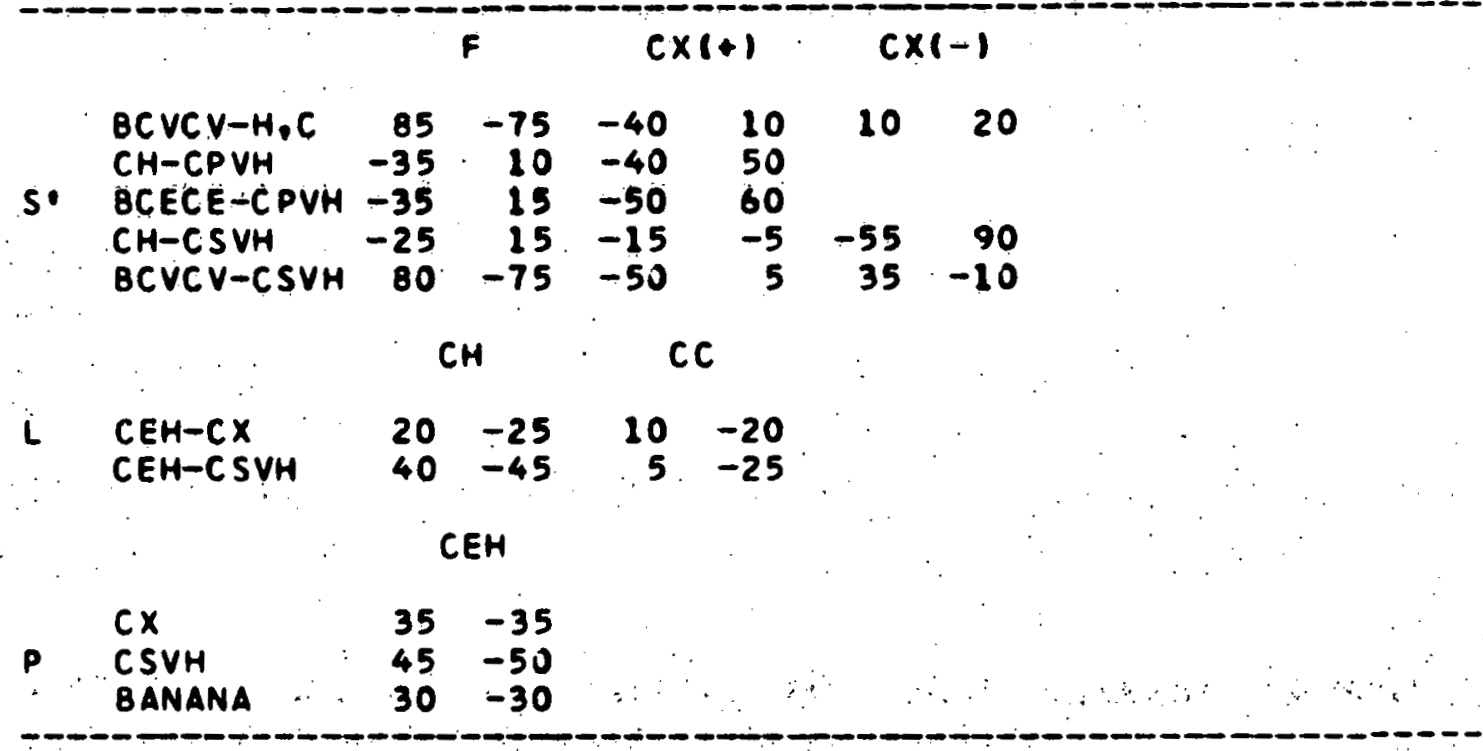


Figure 6. Bond skeletons for fourth neighbor overflow 


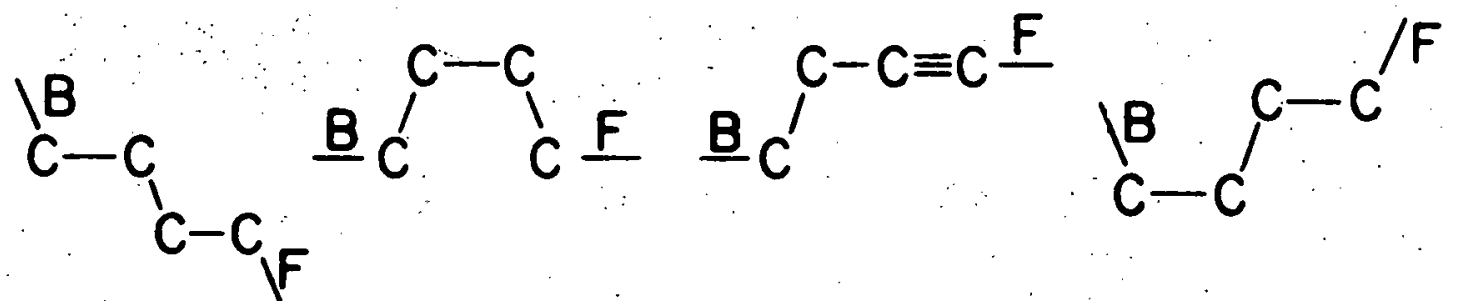

$$
\begin{aligned}
& \text { TT TC TP T T }
\end{aligned}
$$

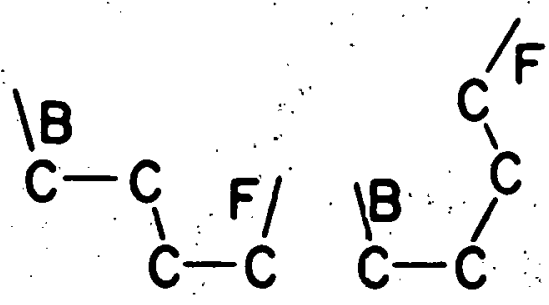

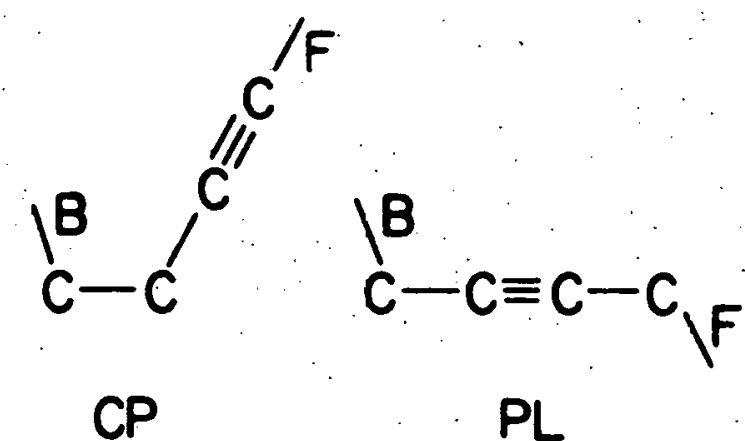

$$
\begin{aligned}
& \text { TT* } \\
& \text { CC }
\end{aligned}
$$

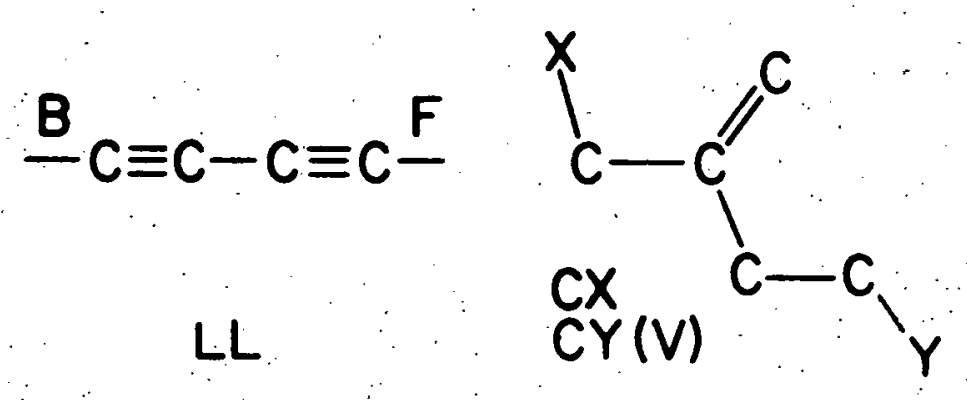


The TT, TC, and TP fourth neighbor fragment coefficients appear in Table 103. The upper column labels $C X$ indicate that the fragment occurring adjacent to the $F$ fragment have coefficients almost independent of their character. The first of the row labels (e.g. $\mathrm{CH}$ in $\mathrm{CH}-\mathrm{h}$ ) characterizes the bond fragment, and the second (e.g. $\mathrm{H}$ in $\mathrm{CH}-\mathrm{H}$ ) characterizes the $F$ fragment.

The CT, CC, and CP fourth neighbor fragment coefficients are listed in Table 104. All labels have the same meaning as in Table 103. This is also true of the PL, LP, and LL fragment coefficients given in Table 105. 
TABLE 103. TT. TC AND TP DVERFLOW ONTO FOURTH NEIGHBOR FRAGMENTS

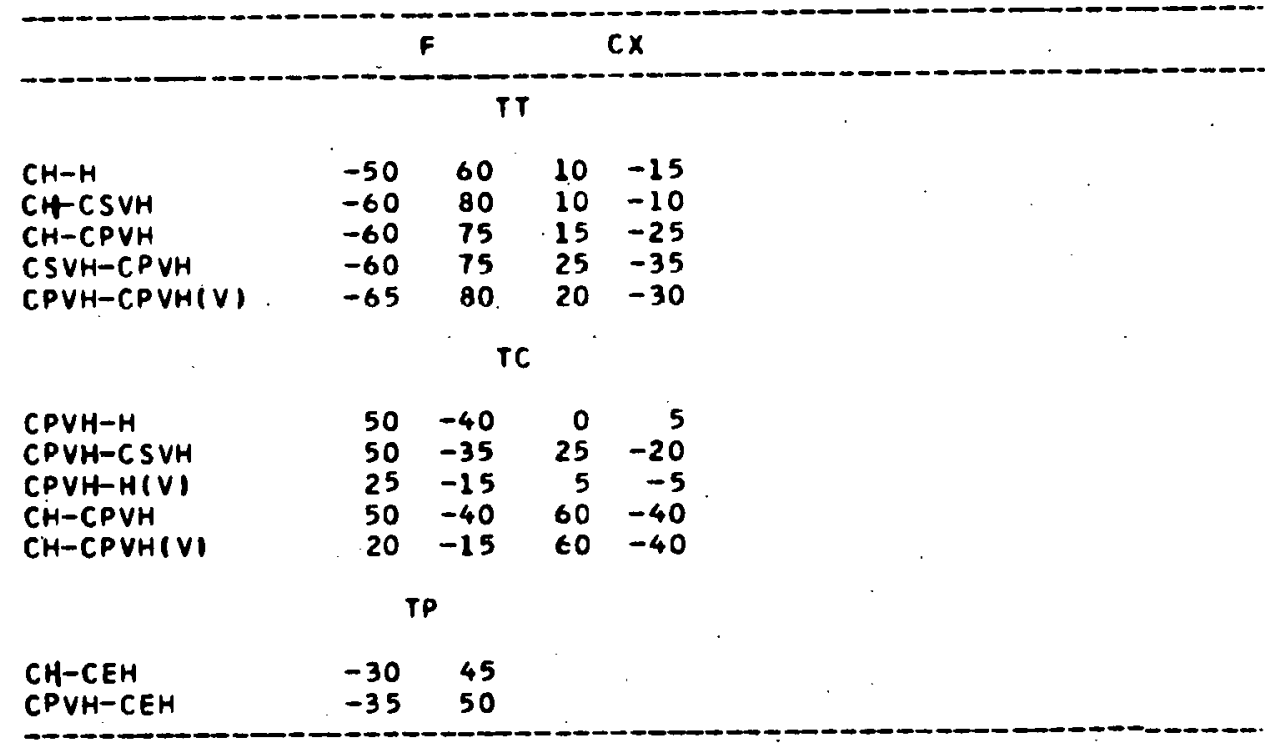


TABLE 104. CT. CC AND CP OVERFLOH CNTO FOURTH NEIGHBCR. FRAGMENTS

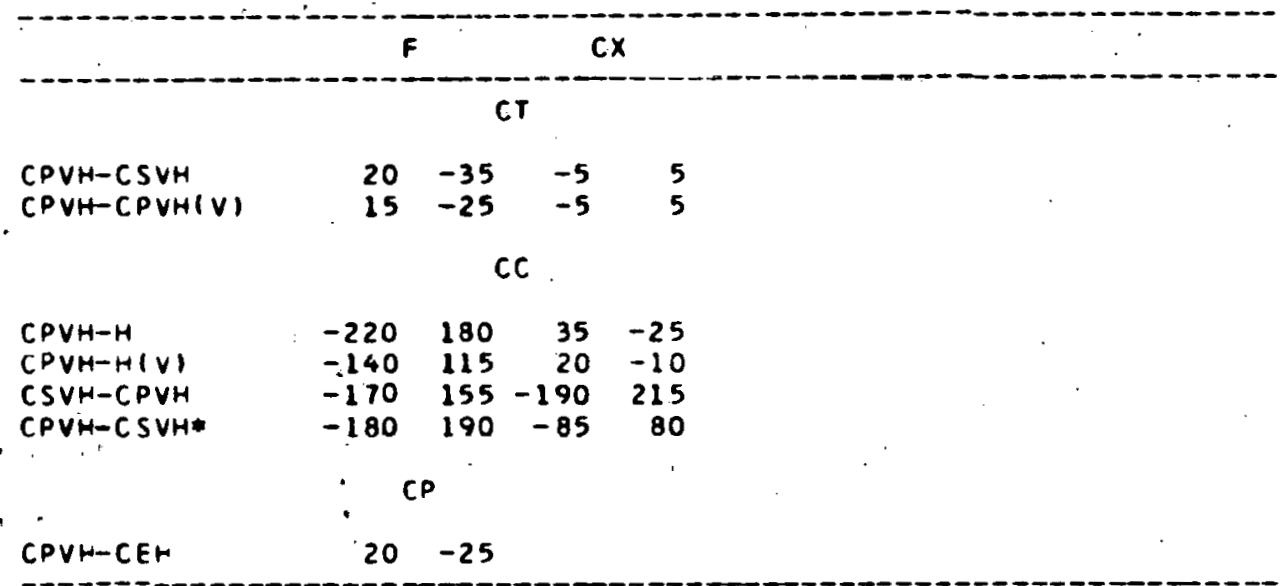


TABLE 105, PL, LP AND LL OVERFLOW ONTO FOURTH NEIGHBOR FRAGMENTS

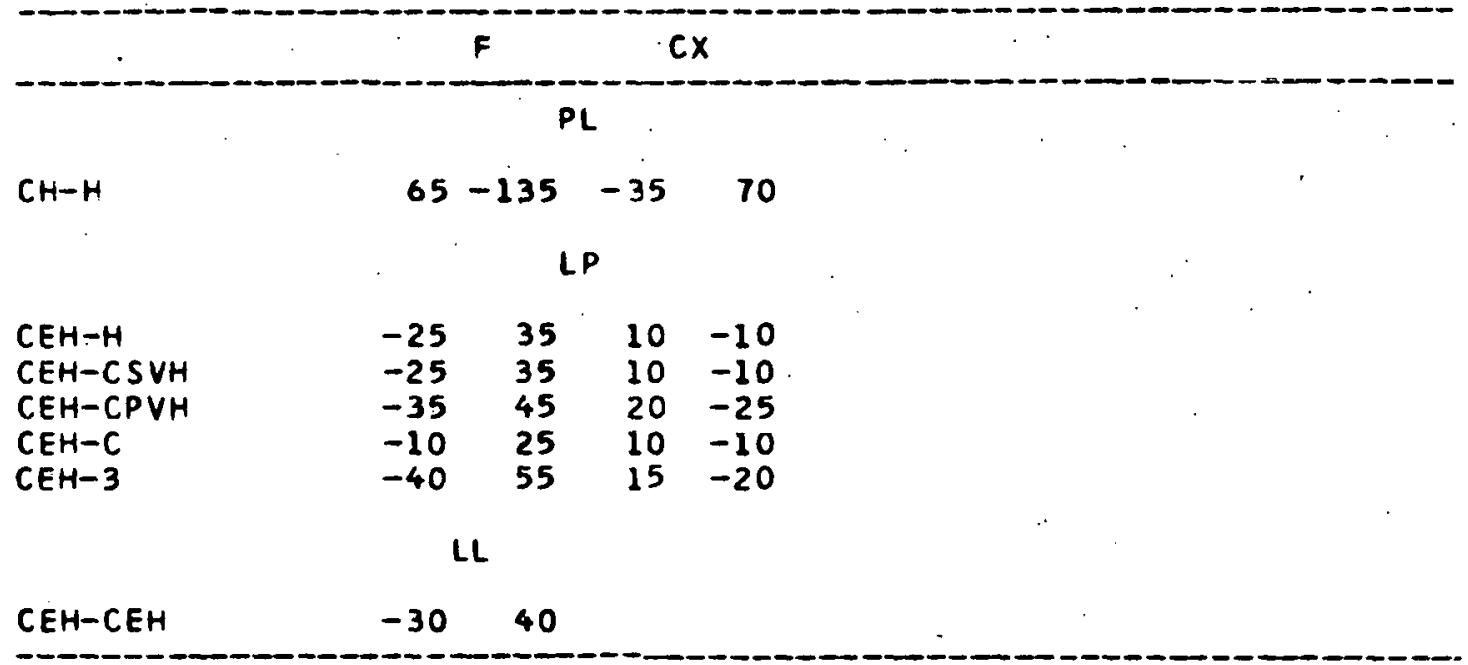


BOND FRAGMENTS AND BOND PROPERTIES

\section{Introduction}

Since more than $95 \%$ of an LMO's population is within the bond fragment, it is natural to wonder if bond properties can be predicted with them. Two such properties that come to mind immediately are bond moments and bond energies, which receive a great deal of attention in the textbooks $(35-37,79,95-101)$. Both have been studied previously in this laboratory $(20)$, where it was found that the former receive important contributions from outside the bond region in an LMO description, and therefore cannot be accurately reproduced with bond fragments alone. The latter, however, were found to stem from contributions within the bond region, and hence bond fragments may be useful for their study. Another property that appears interesting from a bond fragment viewpoint is the nuclear spin coupling constant between directly bonded atoms. Unlike the bond energy, it is possible to make a theoretical argument for this situation (102-105).

\section{Bond Energies}

The concept of 'bond energy' cannot be made non-arbitrary theoretically, so, were it not for its spectacular applications by chemists that have made it a topic for discussion in Introductory courses, there would probably be no urge to postulate its existence. This being the case, the bond energy for present purposes will be defined

$$
E=P(A, B) \cdot e(h y)
$$


where $P(A, B)$ is the bond fragment's bond order, i.e., twlce the product of its coefficients, and $e(h y)$ depends only on the hybridization within the fragment. The latter are determined by equating $E$ to empirical prototype bond energies, e.g. the $e^{\prime} s$ for $s p^{3}-H I s$ and $s p^{3}-s p^{3}$ hybridizations are determined from methane and ethane, respectively. The extensive set of empirical bond atomization energies recently published by Sanderson (106) is used for the present study. These are shown in Table 106 under column A (the remaining column labels designate the overflow fragments) and in Tables 107 and 108 as the first number in each of the row-wise groups. All other numbers in the tables are those obtained with Equation 54. The molecules used to determine the e's are methane $\left(s p^{3}-H\right)$, ethane $\left(s p^{3}-s p^{3}\right)$, ethylene $\left(s p^{2}-H\right.$ and double banana), propene $\left(s p^{2}-s p^{3}\right)$, acetylene $(s p-H)$, propyne $\left(s p-s p^{3}\right)$ and (diatomic) $c_{2}$ (triple banana). The overall agreement is good, especially for the $\mathrm{CH}$ fragments, and isn't particularly dependent on the overflow type. Each overflow fragment lowers the energy around 0.3 to $0.6 \mathrm{kilocalories}$ per mole by its presence. At this level; then, one can obtain reasonable bond energies by assuming that they depend on the bond fragment's hybridization and bond order, the latter being a function of both the bond and overflow fragnent types.

Nuclear Coupling Constants between Directly Bonded Atoms

The classical interaction between the magnetic moment, $m$, of an electron and the magnetic fieid, $B$, of a nucleus (at the origin) is (107)

$$
H=-\underline{m} \cdot \underline{B}
$$


TABLE 106. COMPARISCN OF ALKYL BONO ATOMIZATION ENERGIES AND BONO FRAGMENT ENERGIES IN KILOCALORIES PER MOLE

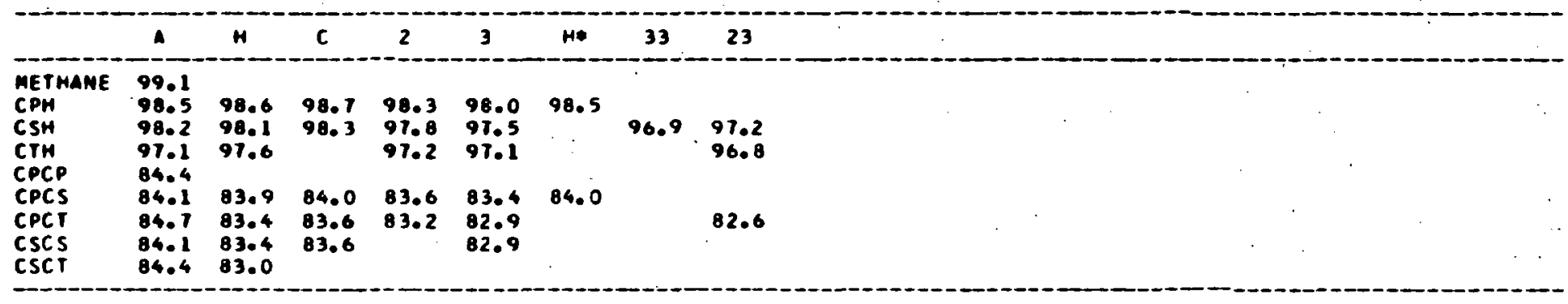


TABLE 107. COMPARISON OF VINYL BOND ATCMIZATION ENERGIES ANO BOND FRAGMENT ENERGIES IN KILOCALORIES PER MOLE

etHYLENE PaOPENE Butadiene 2-BUTENE

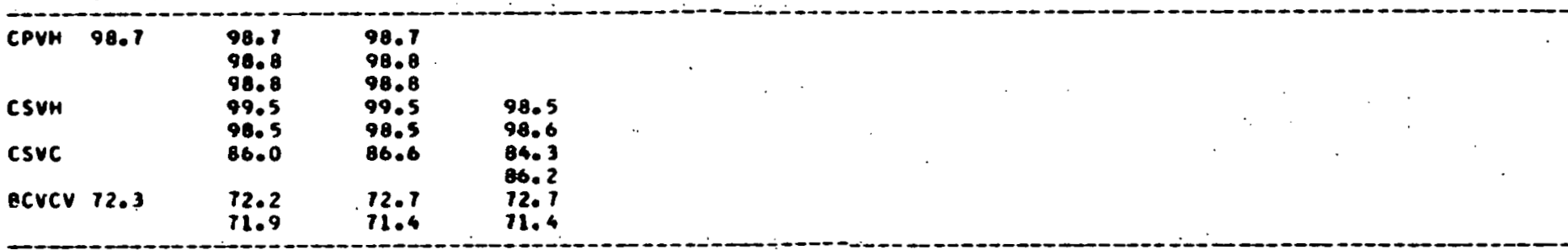


TABLE 108. COMPARISON OF ETHYNYL BOND ATOMIZATION ENERGIES MITH BOND FRAGMENT ENERGIES IN KILOCALORIES PER MOLE

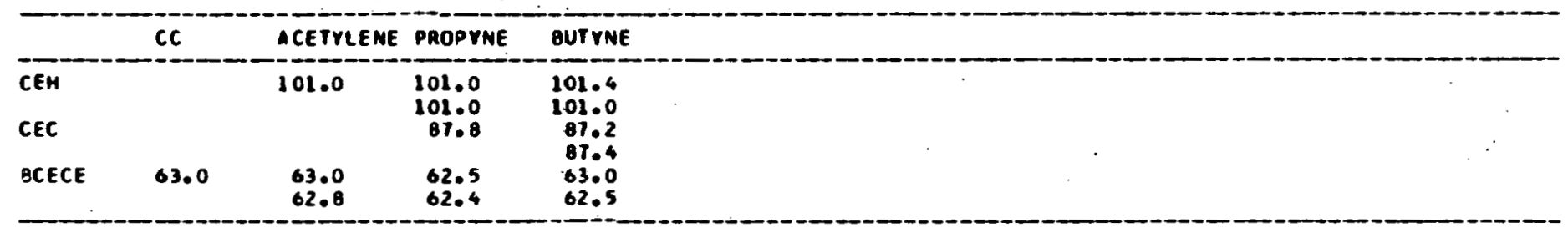


where

$$
B=\operatorname{curl} \underline{A}
$$

and the vector potential $\underline{A}$ is given by (108)

$$
\underline{A}=\underline{M} \times \underline{r} /|\underline{r}|^{3}
$$

with $\underline{M}$ the magnetic moment of the nucleus. This can also be written (108)

$$
\begin{aligned}
H= & -\underline{m} \cdot \nabla \times(\underline{M} \times \nabla(1 / r)) \\
= & (2 / 3)(\underline{m} \cdot \underline{M}) 4 \pi \delta(\underline{r})-[(\underline{m} \cdot \nabla)(\underline{M} \cdot \nabla) \\
& \left.\quad-(1 / 3)(\underline{m} \cdot \underline{M}) \nabla^{2}\right](1 / r) .
\end{aligned}
$$

where $8(r)$ is the Dirac delta function (39). The first term is the Fermi contact interaction $(105,108)$ and the second gives dipole-dipole interactions $(89,105,108)$ which are not important for the coupling between directly bonded atoms $(89,105)$. Thus, the interaction of interest is

$$
V=(2 / 3)(\underline{m} \cdot \underline{M}) \delta(\underline{r}) \text {. }
$$

When many nuclei and electrons are present, this becomes (105)

$$
V=(2 / 3)\left(m_{i} \cdot M_{A}\right) \delta\left(r_{i}-\underline{r}_{A}\right)
$$

with repeated indices summed.

Applying $V$ as a perturbation to a closed shell Born-Oppenheimer wave function gives no first order contribution since the total electronic spin is zero. Second order perturbation theory gives contributions like 
(105)

$$
\langle 0|V| N\rangle\langle N|V| 0\rangle /\left(E_{N}-E_{0}\right), N \neq 0
$$

where $E_{N}$ and $E_{0}$ are the $N$ - and ground-state energies, respectively. Making an average energy approximation (103)

$$
E_{N}-E_{0} \approx E
$$

closure (105) can be used to obtain

$$
\left\langle 0\left|v^{2}\right| 0\right\rangle / E
$$

for the second order contribution to the energy. Therefore

$$
\Delta E=-(4 / 9 E) \underline{M}_{A} \cdot\left\langle 0\left|\delta\left(r_{A}-r_{i}\right) \underline{m}_{i} \underline{m}_{j} \delta\left(r_{j}-r_{B}\right)\right| 0\right\rangle \cdot M_{B}
$$

or in tensor form

$$
\Delta E=\underline{M}_{A} \cdot \underline{K}_{A B} \cdot \underline{M}_{B}
$$

where $\underline{K}_{A B}$ is the second rank reduced coupling tensor (in dyadic form) between nuclei $A$ and $B$. If the molecules are rotating rapidly and randomly, $\underline{K}_{A B}$ may be replaced by its spherical average (105), the reduced coupling constant

$$
\begin{aligned}
K_{A B} & =(1 / 3) \operatorname{trace}\left(\underline{K}_{A B}\right) \\
& =-(4 / 27 E)\left\langle 0\left|\delta\left(\underline{r}_{A}-\underline{r}_{i}\right)\left(m_{i} \cdot \underline{m}_{j}\right) \delta\left(r_{B}-\underline{r}_{j}\right)\right| 0\right\rangle
\end{aligned}
$$

and the interaction energy becomes 


$$
\Delta E=K_{A B}\left(\underline{M}_{A} \cdot \underline{M}_{B}\right)
$$

Thus, in a closed shell molecule with the dipole terms neglected, the interaction between the magnetic moments of the electrons and nuclei leads to a coupling of the nuclear moments, i.e., the electrons couple the nuclear moments.

Introduction of the INDO LCAO-MO closed shell wave function with the approximation that all $\mathrm{AO}^{\prime}$ 's are zero at the nuclear positions except the local s functions gives $(89,105)$

$$
\begin{aligned}
K_{A B}=\left(64 \pi^{2} / 9 E\right) b^{2}\left\langle s_{A}\left|\delta\left(r_{A}\right)\right| s_{A}\right\rangle\left\langle s_{B}\left|\delta\left(r_{B}\right)\right| s_{B}\right\rangle \\
\times(P s(A, B))^{2}
\end{aligned}
$$

where $b$ is the Bohr magneton (109) and $P s(A, B)$ the $s$ bond order between atoms $A$ and $B$. This is the desired expression, showing that the coupling constant between two nuclei is proportional to the square of the $s$ bond order. By replacing Ps $(A, B)$ with $F s(A, B)$; the $s$ bond order of the bond fragment; one obtains

$$
J_{A B} \propto(F s(A, B))^{2} / E
$$

where $J_{A B}$ is the coupling constant which couples the spins directly (rather than the magnetic moments). It is related to the reduced constant by

$$
J_{A B}=\left(\hbar G_{A} G_{B} / 2 \pi\right) K_{A B}
$$


where the G's are nuclear gyromagnetic ratios that include the charge, mass and nuclear $g$ factors $(89,104)$.

In order to use Equation 69, there must be some way of determining the average energies $E$, which have magnitudes near those of the lowest excited states in the electronic spectra. The way of doing this presently will be to assume a value of 13.200 electron volts for ethane (89) and then use the experimental $\mathrm{CH}$ and $\mathrm{CC}$ coupling constants (89) with the theoretical bond orders for ethane, ethylene and acetylene to calculate the E's for the latter two via

$$
E_{2}=\left(F s_{2} / F s_{1}\right)^{2} \cdot\left(J_{1} / J_{2}\right) \cdot E_{1} \cdot
$$

Slightly different values are obtained with the $\mathrm{CH}$ and $\mathrm{CC}$ constants, and the number to be used is the weighted (according to the numbers of bonds) average of these. The results are shown in Table 109, where the calculated J's are those obtained with the weighted averages. (In all that follows, the J's will be in cycles per second (cPs) and the E's in electron volts (ev).) The differences between the calculated and experimental are at worst about $10 \%$

If it is assumed that the ethane, ethylene and acetylene values hold for all alkanes, alkenes, and alkynes, respectively, then it is possible to calculate other coupling constants. A check of this is given in Tabie 110, where the experimental (110) and calculated $\mathrm{CH}$ constants in isobutene, propyne and 2-butyne are compared. The agreement is the same as in Table 109 and shows that the assumption is reasonably valid. 
TABLE 109. AVERAge energies and COUPLing constants in ethane, ethylene and acetrlene

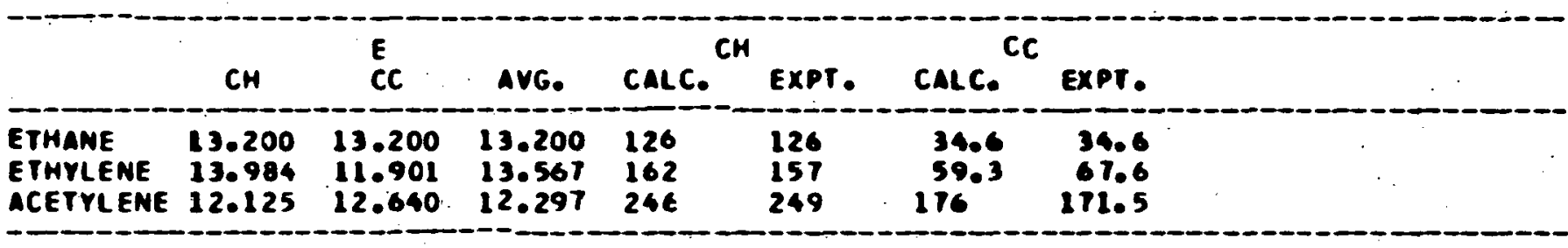


TABLE 110. COMPARISON OF EXPERIMENTAL AND CALCULATEO CH COUPLING CONSTANTS

\begin{tabular}{lllll}
\multicolumn{1}{c}{ CALC. } & EXPT. CALC. & CXPT. \\
\hline ISOBUTENE & 126 & 126 & 163 & 155 \\
PROPYNE & 134 & 132 & 246 & 248 \\
2-BUTYNE & 134 & 131 & &
\end{tabular}


Table III contains some calculated alkyl bond fragment coupling constants for which experimental values aren't known. The upper column labels (cx) characterize the bond fragment, with $x$ defined in the body of the table. The lower column labels characterize the overflow fragment. The row labels specify the type of the alkyl carbon of the bond fragment, e.g. $C X$ with $X=H$ and row label $C P$ means that the bond fragment is $C P H$. Each row label appears three times, according to whether the bond fragment coupling constants apply to alkanes, alkenes or alkynes, respectively. Overall, the behavior is similar to that in Tables 109 and 110: there isn't much sensitivity to the overflow fragment character.

Table 112 lists some vinyl and ethynyl coupling constants (in alkenes and alkynes, respectively) for which no experimental values have been reported. The first seven rows are single bond fragment labels and the last four label banana bond fragments. Note that the column labels have different meanings for single and banana bond fragments. In single bond fragments they characterize the bond fragment, e.g. CEX with $X=H$ means that the bond fragment is ethynyl. $\mathrm{CH}$. In banana bond fragments they characterize the overflow fragment, e.g. $X=H$ means the overflow fragment is $\mathrm{CH}$. The cis or trans and di in parentheses refer to primary vinyl $\mathrm{CH}$ bond fragments cis or trans to monosubstituents and in disubstituted ethylenes. Here, too the behavior is similar to Tables 109 and 110 ; there being little sensitivity to the overflow fragment type.

Some coupling constants for systems having conjugate banana bonds are shown in Table 113. The first column label is the average energy used for the molecule. These are the previous values except for vinyl acetylene, 
TABLE 111. CALCULATEO ALKYL COUPLING CONSTANTS

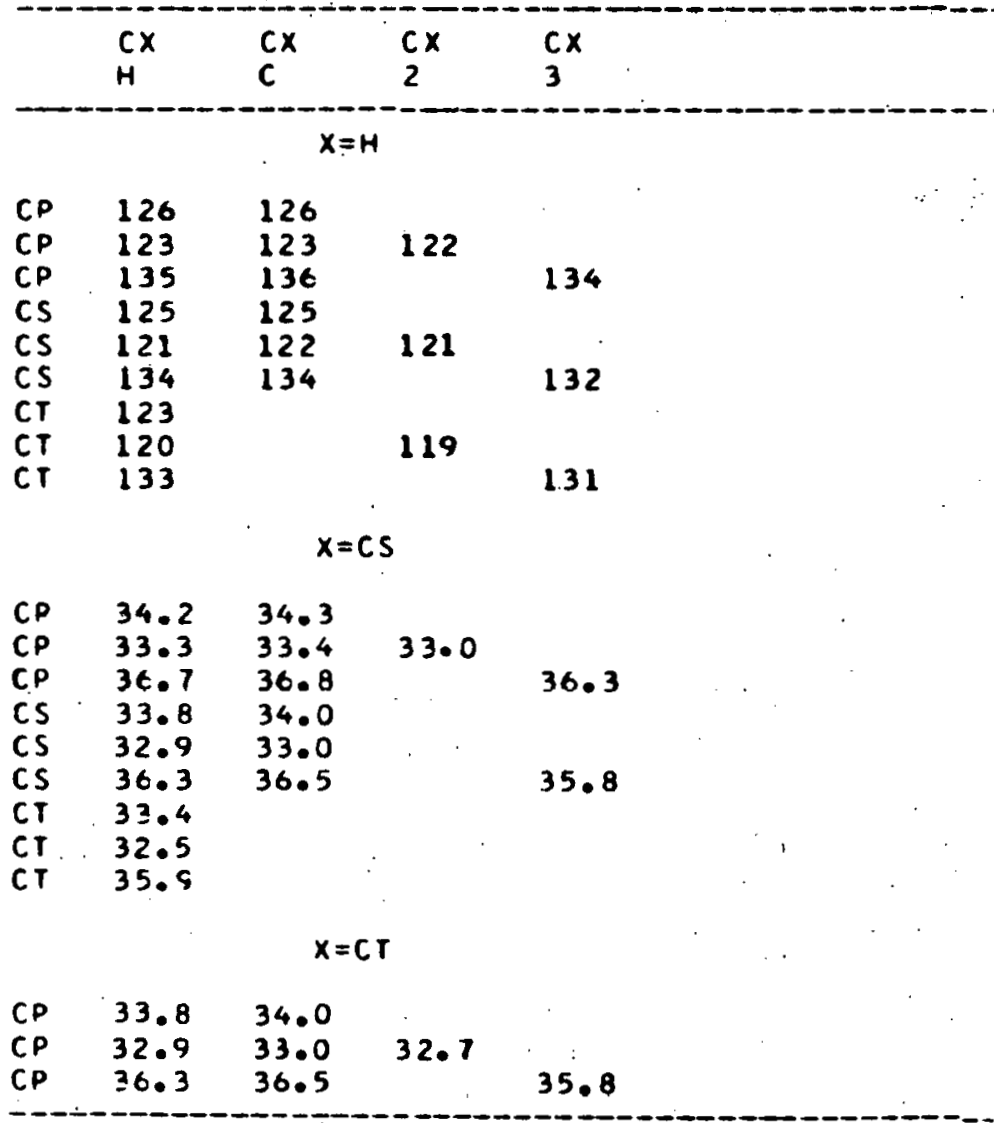


TABLE 112. CALCULATED VINYL AND ETHYNYL COUPLING CONSTANTS

\begin{tabular}{|c|c|c|c|c|}
\hline$x=$ & $\mathrm{H}$ & $C P$ & CS & $C T$ \\
\hline $\begin{array}{l}\text { CPVX(CIS) } \\
\text { CPVXITRANS) } \\
\text { CPVXIDII } \\
\text { CSVX-H } \\
\text { CSVX-C } \\
\text { CIVX-H } \\
\text { CEX } \\
\text { BCPVCSV-X } \\
\text { RCSVCSV-X } \\
\text { ECPECSE-X } \\
\text { BCSVCSV-X }\end{array}$ & $\begin{array}{l}162 \\
162 \\
163 \\
161 \\
162 \\
\\
246 . \\
58.6 \\
57.8 \\
175 \\
173\end{array}$ & $\begin{array}{l}44.5 \\
44.7 \\
44.1 \\
66.7 \\
58.8 \\
175\end{array}$ & $\begin{array}{l}44.0 \\
44.1 \\
66.1\end{array}$ & $\begin{array}{l}43.6 \\
65.2\end{array}$ \\
\hline
\end{tabular}


TABLE 113. VINYL ANC ETHYNYL COUPLING CONSTANTS IN SOAE CONJUGATED SYSTEMS

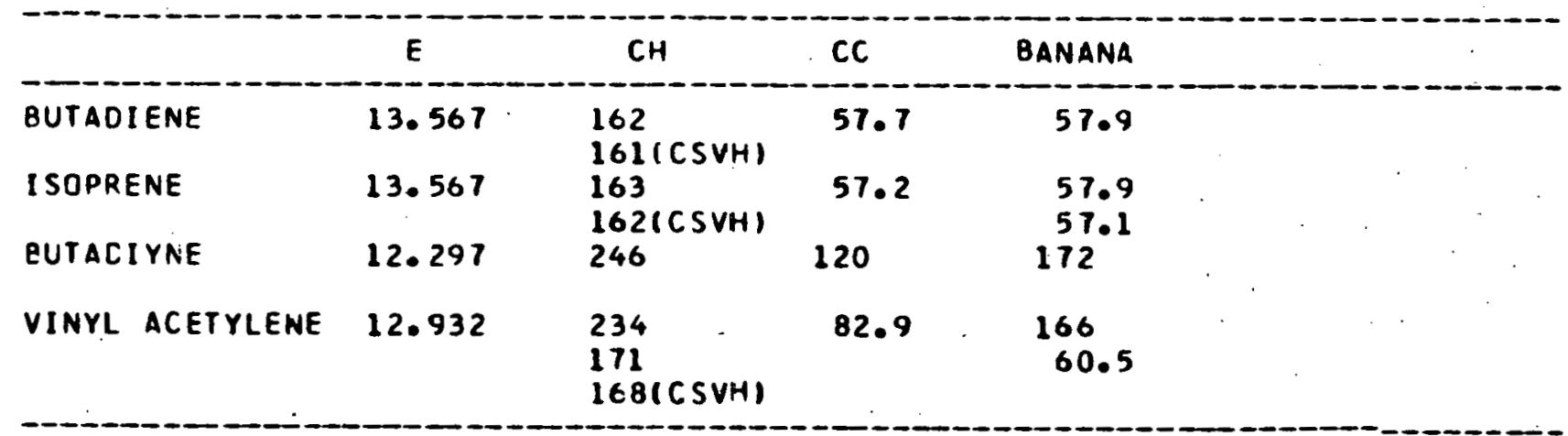


where an average of the ethylene and acetylene values was used. The remaining column labels characterize the bond fragment. The CSVH in parentheses distinguishes the secondary vinyl $\mathrm{CH}$ bond fragment from that of the primary vinyi $\mathrm{CH}$. In vinyl acetylene, the first of the three $\mathrm{CH}$ bond fragment entries is for the ethyny $\mathrm{CH}$ bond. The $\mathrm{CC}$ bond fragments in the first two rows (butadiene and isoprene) are those for vinyl carbonvinyl carbon single bonds. The CC bond fragments in the last two rows (butadiyne and vinyl acetylene) are for ethynyl carbon-ethynyl carbon and ethynyl carbon-vinyl carbon bond fragments, respectively. The banana bond fragment entries for isoprene are for the primary vinyl-secondary vinyi and primary vinyl-tertiary vinyl cases, respectively, whi le those for vinyl acetylene are for triple and double bonds, respectively.

Even in conjugated systems, we see that the greatest sensitivities are to the average energy and bond fragment character: the effects of conjugation are small. 


\section{BENT BONDING}

\section{Introduction}

Early LMO $(16,17)$ and related $(30,31)$ works have shown that orbital centroids do not point directly along the bond skeleton, i.e., that bonds are 'bent.' The concept is normally applied to only slightly bent bonds, and not to banana bonds, where the bending is largely due to the availability of several electron pairs for bonding between the same two atoms. It will be shown here that this slight bending occurs because of the LMO delocalization onto the geminal fragment near (relative to the bond fragment) hybrids and hence is an inherent property of the solutions to the LCAO-MO-SCF equations (87).

\section{Bent Bonds and Geminal Fragments.}

All non-banana carbon hybrids have centroids lying directly along the bond skeketon. We consider these to be unit vectors along the bond skeleton that are centered on their associated carbon atoms and point away from them. Consider a LMO whose bond fragment hybrid is associated with rarbon atom $Q$. Let $h$, and $a, b, c$, be the coefficients of its bond fragment and three geminal fragment near hybrids, respectively, on atom Q. We define vectors $\underline{h}$, and $\underline{a}, \underline{b}, \underline{c}$ as being proportional to their associated hybrid centroid unit vectors with proportionality constants $h$ and $a, b, c$, respectively. Thus $\underline{h}$ and $\underline{a}, \underline{b}, \underline{c}$ have magnitudes equal to the magnitudes of the coefficients $h$ and $a, b, c$ of the LMO bond and geminal fragments on atom $Q$. The vectors $\underline{a}, \underline{b}, \underline{c}$ may have the same or opposite directions as their associated centroid vectors (according as $a, b, c$, are positive or 
negative).

The bending angle $A$ of the bond fragment on atom 0 is defined to be the angle between $\underline{h}$ and $\underline{k}$, where

$$
\underline{k}=\underline{h}+\underline{a}+\underline{b}+\underline{c} \text {. }
$$

Thus

$$
A=\arccos (\underline{k} \cdot \underline{h} / k h)
$$

Equations 72 and 73 show the connection between bent bonding and geminal near hybrid delocalization. The larger and more unsymmetric the latter (relative to the bond fragment), the greater is the deviation from the bond skeleton.

Table 114 lists some values of $A$ for the bond fragments having $C H$ overflow fragments that were encountered is this work. All angles have been rounded to the nearest half degree. It is sufficient to specify the character of carbon atom $Q$ (row labels) and the character of the other atom of the bond fragment (column labels). Table ils lists the sensitivity of the bending angle to overflow fragment (column labels) changes in primary $\mathrm{CH}$ bond fragments. Deviations from the angle observed for the $\mathrm{CH}$ overflow fragment case are typical of all bond fragments. 


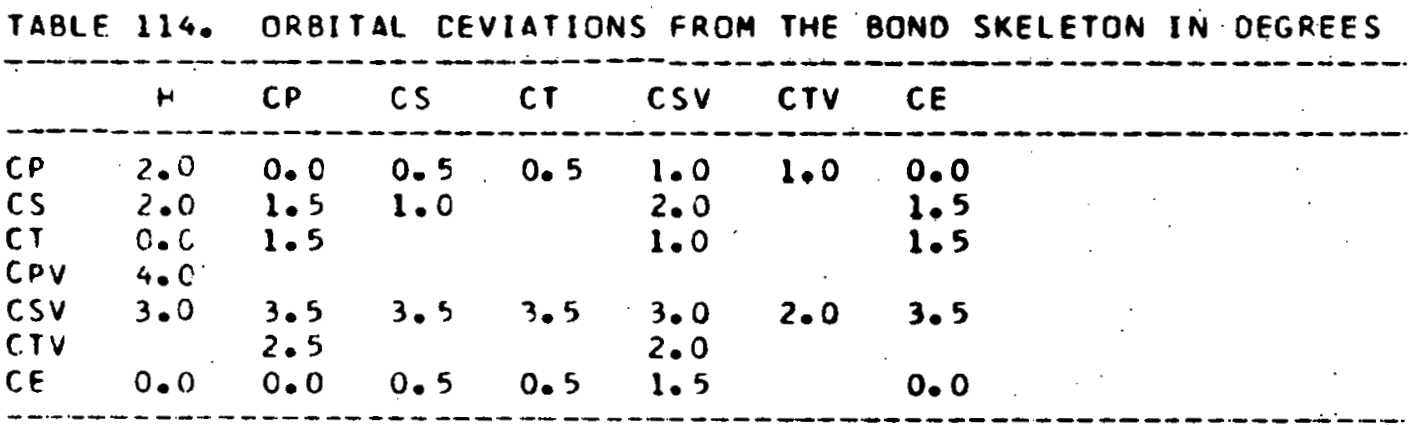


TABLE 115. PRIMARY CH ORBITAL DEVIATIONS FROM THE BOND SKELETON IN OEGREES

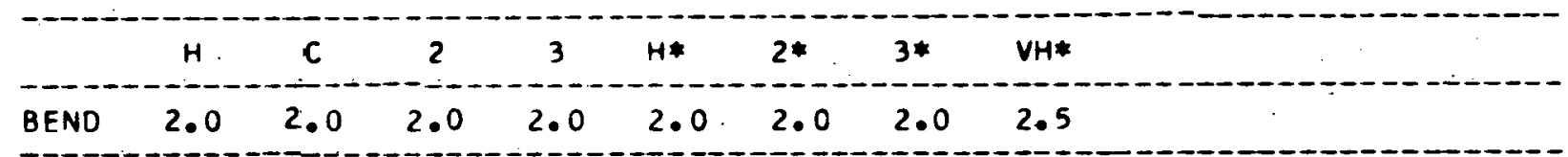


CONJUGATION AND HYPERCONJUGATION

Traditionally, conjugation has been reserved for molecules having alternating double bonds $(56,57,59,111,112)$, these being common and having unique, often spectacular properties which are readily accessible to theoretical and experimental analysis. Presumably recognizing that the phenomenon involved the interaction of bonds separated by one bond region (next neighbor bonds), Mulliken $(113,114)$ generalized the concept to other systems, calling it hyperconjugation. Since then, it has been widely appreciated in organic chemistry (79) that hyperconjugation and conjugation arise from the same fundamental factor: delocalization of electrons.

Recalling the perturbation theory results given earlier in Table 35 , it follows that interactions among the bond fragments (considered as twocenter localized orbitals) lead to vicinal fragment coefficients in close agreement with the vicinal fragment coefficients of the actual LMO's. If one makes the reasonable assumption that these interactions are a measure of hyperconjugation, then one expects hyperconjugation effects to be manifest in the following two ways: (1) by variations in the bond fragment coefficients that are induced by changing the vicinal group, and (2) by variations in the vicinal group coefficients that are induced by changing the bond fragment. The former hyperconjugation effect amounts to changing the overflow fragment in most cases. The latter has already been shown to account for hindered rotation origins $(18,19,69,70)$.

Since a given overflow fragment type was found to introduce simi lar coefficient variations in most bond fragments (see Tables 40 and 46 ), it is reasonable to think that it will also produce similar variations in 
bond (fragment) properties, other things being equal. This can be seen in Tables 106-8, where each hybridization type displays a characteristic bond energy, around which similar deviations occur, depending on the overflow fragments. It is interesting that in this context the hybridization effects are typically two to three times larger than the hyperconjugation effects, since this is a controversial topic $(46,79,115)$. 


\section{EMPIRICAL OBSERVATION OF LOCALIZED BONDS}

The November 22, 1971, issue of Physical Review Letters reported the results of some conceptually simple and fundamentally important experiments (116): The Compton X-ray scattering technique was used to directly observe localized bonding in some hydrocarbons. This 'direct' observation is the outstanding contribution of these experiments; for previous experimental evidence for 'observables' in the localized representation has been indirect, e.g. the usual notions of bond energies, moments, etc.

It can be shown (117) that the cross section for compton scattering by atoms or molecules (in atomic units) is

$$
\sigma=2 \pi\left(\underline{\epsilon} \cdot \underline{\epsilon}^{\prime}\right)^{2} \omega^{\prime} / \omega\left(\mathrm{d} l / c^{4}\right) J(l)
$$

where the primes refer to the outgoing rays, the $\underline{\epsilon}^{\prime} s$ are polarization directions, $\ell$ is the reduced wavelength, or distance from the Compton peak $(118,119)$, and

$$
J(l)=\frac{1}{2} \Sigma \int I_{L}(p) d p / p
$$

is the 'Compton profile.' The quantity $I_{L}(p)$ is the radial momentum density for orbital L, and the sum in Equation 75 is over the occupied MO's and the integration from $|\ell|$ to infinity.

Generally, it is impossible to measure the individual 1; however, by subtracting out the contributions of inner shell electrons and judiciously choosing pilot systems, Eisenbarger and Marra (116) were able to observe them. Methane gave the $\mathrm{CH}$ bond compton profile, and values for single 
and double CC bonds (no triple bonds were studied) followed from their experimental ethane and ethylene profiles, respectively, by assuming a constant $\mathrm{CH}$ value. Ușing these numbers, they were able to calculate values agreeing with experiment within experimental accuracy (1-5\%) for several other hydrocarbons, thus providing strong proof both for the validity of Equation 75 and the existence and transferability of 'bond' $\underline{J}$, and hence localized bonding itself.

Prior to this experiment, but still quite recently, a comparative study of some hydrocarbon LMO's in the momentum representation was reported which used ab initio minimal basis LCAO-SCF-MO theory $(24,118)$. There, too, a good deal of transferability was observed, not only for compton profiles, but for momentum density contours and expectation values also. In fact, the theoretical results concluded that hydrocarbon compton profiles can be well represented by using only three values, one for inner shells, another for all CH bonds, and still. another for all CC bonds. It should be pointed out, however, that their building block total profile calculation for benzene, which presumably tests the last assumption, gave generally poorer agreement with the SCF results than did their similar cyclopropane. calculation. Especially in view of the fact that Compton profiles are not one of the more sensitive properties to errors in the wave function (119), it may be that too much transferability is being assigned. For example, the highly bent ethylene banana bonds $(50$ degrees above and below the bond axis) would be expected to increase the Compton profiles at $l=0$ (which is the inverse momentum expectation value) from its ethane $C C$ bond value (0.412). In fact, division of the ethane value by the sine of $50^{\circ}$ does 
give a value (about 0.54 ) rather close to the ethylene double bond value $(0.516)$. 


\section{CANONICAL AND VIRTUAL ORBITALS}

\section{Introduction}

These orbitals have several properties interesting enough to call for some way of determining them from the localized orbitals. Chief among these are that they approximate the selection rules for electronic transitions (44) and provide estimates of the lower excited states of the electronic spectrum (34). To determine them, all that is actually needed is the knowledge that the CMO's diagonalize the fock matrix (14); but this implies that they are symmetry orbitals (14), l.e., carrier functions (82) for the irreducible representations of the molecule's point group. This can be exploited in many cases to shorten their computation.

\section{Virtual Orbitals}

The virtual, or unoccupied, MO's (VMO's) are those produced in any closed-shell LCAO-SCF-MO calculation having more basis functions than electron pairs. They can be used, in canonical form, to approximately describe the electronic spectrum and, in any form, to calculate the so-called polarizabilities useful in the applications of perturbation theory to Mo wave functions (87-93). It is therefore desirable to compute them as simply as possible as well as having some way of casting them in canonical form. The latter will be given in the next section.

The simplest way to calculate VMO's from LMO's (87) makes use of the notion of antibonding (100). Let $c$ and $c^{\prime}$ be bond fragment coefficients for hybrids $h$ and $h^{\prime}$, respectively, in some LMO $\lambda$. The INDO antibonding virtual localized orbital is defined 


$$
\lambda^{\prime}=\left(c^{\prime} h-c h^{\prime}\right) /\left(c^{2}+c^{\prime 2}\right)^{1 / 2}
$$

and, in the paraffins, are in one-to-one correspondence with the occupied LMO's. The $\lambda^{\prime}$ are mutually or thogonal, but only approximately orthogonal to the occupied space. To make them so orthogonal, some process, e.g. Gram-Schmidt orthogonalization (120), should be used which doesn't change the occupied space, as this is the one accurately modeled. When this has been done, one has a set of virtual LMO's (VLMO's).

\section{Canonical Orbitals}

In the present context, one should also determine CMO's for the occupied and virtual spaces without altering the former. Thus, let the $\lambda$ 's be LMO's or VUMO's and let

$$
\lambda_{i}=\Sigma T_{\mu} \mathbf{f}_{\mu}
$$

be their expansions in symmetry orbitals. Using standard projection operators (82) $P_{\mu}$ for each irreducible representation, one can project the set

$$
\bar{\mu}_{\mathbf{i}}=\mathbf{P}_{\mu} \lambda_{\mathbf{i}}=\mathbf{T}_{\mathbf{i}_{\mu}} \mathbf{f}_{\mu}
$$

from each $\lambda$, and by Gram-Schmidt orthogonalization (120), obtain a set of $\mathrm{N}_{\mu}$ linearly independent symmetry MO's $\mu$ within the space in question. Since the Fock operator depends only on the occupied space (6), it is known and its matrix elements, $F(\mu ; i j)$ in the $\mu$ basis can be calculated. The CMO's of symetry $\mu$ are the eigenfunctions of this $N_{\mu}$ by $N_{\mu}$ matrix, 
and repeating the process for all symmetries produces all of the CMO's. When a given symmetry occurs only once, $N_{\mu}$ is unity and the $\mu_{i}$ is the CM0. At the other extreme, when there is no symmetry, there are no short cuts and the full fock matrix in the occupied and/or virtual spaces must be diagonalized. In this situation, one may consider another option, especially for smaller molecules, namely, diagonalizing the Fock matrix in the atomic basis and simultaneously obtaining the occupied and virtual CMO's. 
MODEL MOLECULES

Perhaps the most important consequence of this work is that it specifically describes what must be done to obtain truly transferable localized molecular orbitals when one has a set of transferable bond lengths and bond angles. Thus, one is provided with a prescription (as opposed to a set of equations) for assembling model molecules. Ultimately, this would be done with a computer. Starting from minimal input data, the entire molecule could be assembled from internal access information and tables. Options could be provided to furnish the user with canonical molecular orbitais, virtual molecular orbitals, and any number of molecular properties. This latter has already been done for diatomic molecules by A. C. Wahl et al. (121).

There are less accurate ways to calculate model localized orbitals beginning from SMO's or PMO's such as were given in Table 35 . They are based upon the fact that both sets of orbitals have coefficients in close agreement with the LMO's, except for the geminal fragments. One could therefore replace the SMO and PMO geminal fragment coefficients wi th model values to obtain representations of the LMO's.

However, to obtain the SMO's one must have the canonical MO's, and to obtain the PMO's one must essentially model the bond fragment coefficients (to serve as unperturbed functions). As a result, neither method offers a technical advantage to the one which would use the computer catalog. 
APPENDIX: INTERPRETATIONS OF LMO'S

For any single determinant, closed-shell, MO wave function, the electron repulsion energy is (6)

$$
R=D+C-X,
$$

where $D$ is the 'self-energy'

$$
D=\Sigma\left[i^{2} \mid i^{2}\right]
$$

C is the Coulombic repulsion energy

$$
C=2 \Sigma \Sigma^{\prime}\left[i^{2} \mid j^{2}\right]
$$

and $X$ is the exchange repulsion energy

$$
x=\Sigma \Sigma^{\prime}[i j \mid i j]
$$

All sums are over the occupied.MO's and the primes mean that the $i=j$ terms are omitted from the sum. The LMO's are those which simultaneously maximize $D$ and minimize $C$ and $X$, of course leaving $R$ invariant. Maximization of $D$ means that the LMO's are those which, on the average, are the most highly concentrated MO's possible energy-wise. Minimization of C means that they are also the MO's whose average long-range, or coulombic, interactions are as small as possible. Finally, minimization of $X$ means that their short-range, or exchange interactions are likewise as small as possible (on the average). Succinctly, energy localization accomplishes three things: concentration of the MO's, long-range separation of different MO's, and short-range separation of different $\mathrm{MO}^{\prime} \mathrm{s}$ (22). 
If it were possible to reduce $x$ to zero, one would have instead of Equation 79

$$
R=D+C,
$$

which is the repulsion energy for a Hartree product wave function (122). The Hartree product for a closed-shell takes the Pauli principle into account by doubly occupying each MO, but does not include that part of the principle which requires total antisymmetry of the wave function. Thus, each electron can be identified with a specific Mo and is not exchanged. Since it is general iy impossibie to reduce $x$ to zero, one doesn't realize this situation, but the LMO's are those MO's for which $R$ most nearly approaches the Hartree form, i.e., they are proper quantum mechanical orbitals which identify with specific electrons as closely as possible.

Another interpretation of localized bonding follows from some works done more than twenty years ago which dealt with electron probability distributions and their maxima in atoms (123-125). Single-determinant orbital wave functions were made and the analytical form of their square determined after performing the spin integrations. This was then maximized with respect to all spatial coordinates and the resulting centroids determined. By considering the

$$
1 s^{2} 2 s \quad 2 p x 2 p y \quad p z
$$

atomic state of carbon, tetrahedral valence hybrids and inner shells were predicted when all AO's were allowed to mix, and trigonal hybrids obtained when one of the PAO's was witheld. Thus, the basis functions which, when 
perturbed, may be regarded as giving rise to the LMO's, are those whose centroids maximize the atomic spatial electron probability distribution, and it is very likely that LMO centroids similarly maximize the molecular spatial electron probability distribution. 


\section{LITERATURE CITED}

1. Kekulé, A. Untersuchungen ïber aromatische Verbindungen. Annalen der Chemie 137, 129 (1866).

2. Japp, F. R. Kekulé memorial lecture. Trans. Chem. Soc. (London) 73 , 97 (1898).

3. Lewis, G. N. Valence and the structure of atoms and molecules. New York, N.Y., The Chemical Catalog Co. 1923.

4. Langmuir, 1. The constitution and fundamental properties of solids and liquids. Part 1. Solids. J. Am. Chem. Soc. 38, 2221 (1916).

5. Edmiston, C. and Ruedenberg, K. Localized atomic and molecular orbitals. Rev. Mod. Phys. 35, 457 (1963).

6. Edmiston, $C$. and Ruedenberg, $K$. Localized atomic and molecular orbitals. 11. J. Chem. Phys. 43, 597 (1965).

7. Edmiston, C. and Ruedenberg, K. Localized atomic and molecular orbitals. IIl. In Löwdin, P. 0., ed. Quantum theory of atoms, molecules and the solid state. New York, N.Y., Academic Press. 1966.

8. Ruedenberg, K. Localized self-consistent-field orbitals in atoms and molecules. In Sinanoglu, 0. , ed. Modern quantum chemistry. Vol: 1. New York, N.Y., Academic Press. 1965.

9. Pople, J. A. and Segal, G. A. Approximate self-consistent molecular orbital theory. 1. Invariant procedures. J. Chem. Phys. 43, S 129 (1965).

10. Pople, J. A. and Segal, G. A. Approximate self-consistent molecular orbital theory. II. Calculations with complete neglect of differential overlap. J. Chem. Phys. 43, S136 (1965).

11. Pople, J. A. and Segal, G. A. Approximate self-consistent molecular orbital theory. 111 : $C N D O$ results for $A B_{2}$ and $A B_{3}$ systems. J. Chem. Phys. 44, 3289 (1966).

12. Pople, J. A., Beveridge, D. L., and Dobosh, P. A. Approximate selfconsistent molecular orbital theory. $V$. Intermediate neglect of differential overlap. J. Chem. Phys. 47, 2026 (1967).

13. Pople, J. A. and Beveridge, D. L. Approximate molecular orbital theory. New York, N.Y., McGraw-Hill Book Co., Inc. 1970. 
14. Roothaan, C. C. J. New developments in molecular orbital theory. Rev. Mod. Phys. 23, 69 (1951).

15. Fock, V. Näherungsmethode zur Lösung des quantenmechanischen Mehrkörperproblems. Z. Physik 61, 126 (1930).

16. Rothenberg, S. Localized orbitals for polyatomic molecules. 1. The transferability of the $\mathrm{C}-\mathrm{H}$ bond in saturated molecules. J. Chem. Phys. 51, 3389 (1969).

17. Rothenberg, S. Localized orbitals for polyatomic molecules. II. The $\mathrm{C}-\mathrm{H}$ bond transferability in unsaturated systems. J. Amer. Chem. Soc. 93,68 (1971).

18. England, W. and Gordon, M. S. Localized charge distributions 1 . General theory, energy partitioning, and the internal rotation barrier in ethane. J. Am. Chem. Soc. 93, 4649 (1971).

19. England, W. and Gordon, M. S. Localized charge distributions. 11 . An interpretation of the barriers to internal rotation in $\mathrm{H}_{2} \mathrm{O}_{2}$. J. Am. Chem. Soc. 94, 4818 (1972).

20. England, W. and Gordon, M. S. Localized charge distributions. III. Transferability and trends of carbon-hydrogen moments and energies in acyclic hydrocarbons. J. Am. Chem. Soc. 94, 5168 (1972).

21. England, W. and Ruedenberg, K. Localized $\pi$-orbitals, Pauling bond orders, and the origin of aromatic stability. Theor. Chim. Acta, 22, $196(1971)$.

22. England, W., Salmon, L. S., and Ruedenberg, K. Localized molecular orbitals: a bridge between chemical intuition and molecular quantum mechanics. Fortschr. Chem. Forsch. 23, 31 (1971).

23. Ruedenberg, K. Hindered rotation, Hellmann-Feynmann theorem, and localized molecular orhitals. J. Chem. Phys. 4l, 588 (1964).

24. Epstein, 1. R. Molecular momentum distributions and Compton profiles. 11. Localized orbital transferability and hydrocarbons. J. Chem. Phys. 53, 4425 (1970).

25. Kaldor, U. Localized orbitals for $\mathrm{NH}_{3}, \mathrm{C}_{2} \mathrm{H}_{4}$, and $\mathrm{C}_{2} \mathrm{H}_{2}$, J. Chem. Phys. 46, 1971 (1967).

26. Kaldor, U. and Shavitt, I. LCAO SCF computations for ammonia. J. Chem. Phys. 45, 888 (1966).

27. Stevens, R. M. ACcurate SCF calculation for ammonia and its inversion motion. J. Chem. Phys. 55, 1725 (1971). 
28. Rauk, A., Allen, L. C., and Clementi, E. Electronic structure and inversion barrier of ammonia. J. Chem. Phys. 52, 4133 (1970).

29. England, W. and Gordon, M. S. The ammonia geometry. Unpublished paper. Ames, lowa, Department of Chemistry, lowa State UnIversity. 1972.

30. Veillard, A. and Del Re, G. Hybridization in cyclopropane, cyclobutane and cubane. Theor. Chim. Acta 2, 55 (1964).

31. Del Re, G., Esposito, U., and Carpentieri, M. Bent bonds, hybridization, and the maximum localization criterion. Theor. Chim. Acta 6, 36 (1966).

32. McWeeny, R. and Del Re, G. Criteria for bond orbitals and optimum hybrids. Theor. Chim. Acta 10, 13 (1968).

33. Magnasco, V. and Musso, G. F. On factors contributing to rotational barriers. Chem. Phys. Lett. 9, 433 (1971).

34. Daude 1, R., Lefebvre, R., and Moser, C. Quantum chemi.stry. New York, N.Y., Interscience Pub., Inc. 1959.

35. Pauling, L. The nature of the chemical bond. 3rd ed. I thaca, N.Y., Cornell University Press. 1960.

36. Syrkin, Y. K. and Dyatkina, M. E. Structure of molecules and the chemical bond. New York, N.Y., Dover Publications, Inc. 1964.

37. Kondratyev, V. The structure of atoms and molecules. New York, N.Y., Dover Publications, Inc. 1965.

38. Platt, J. R. The chemical bond and the distribution of electrons in molecules. In Flügge, S. ed. Encyclopedia of physics. Vol. XXXVII/2. Molecules 2. Berlin, Springer-Verlag. 1961.

39. Dirac, P. A. M. The principles of quantum mechanics. 4th ed. London, Oxford University Press. 1958.

40. Pullman, A. and Pullman, B. Les theories electronique de la chimie organique. Paris, Masson et Cie. 1952.

41. Parr, R. G. Quantum theory of molecular electronic structure. New York, N.Y., W. A. Benjamin, Inc. 1963.

42. Streitweiser, A. Molecular orbital theory for organic chemists. New York, N.Y., John Wiley $\&$ Sons, Inc. 1961.

43. Roberts, J. D. Notes on molecular orbital calculations. New York, N.Y., W. A. Benjamin, I nc. 1962. 
44. Ballhausen, C. J. and Gray, H. B. Molecular orbital theory. New York, N.Y., W. A. Benjamin, Inc. 1965.

45. Pilar, F. Elementary quantum chemistry. New York, N.Y., McGrawHi 11 Book Co. 1968.

46. Dewar, M. J. S. The molecular orbital theory of organic chemistry. New York, N.Y., McGraw-Hill Book Co. 1969.

47. Gordon, M. S. and Pople, J. A. MBLD: Standard geometric models and cartesian coordinates of molecules. Quantum Chemistry Program Exchange News letter 24, 28 (1969).

48. Hinze, J. Heteropolar bonds. In Eyring, H., ed. Physical chemistry. Vol. V. Valency. New York, N.Y., Academic Press. 1970.

49. Hund, F. Zur Deutung der Molekelspektren. IV. Z. Physik 5l, 759 (1928).

50. Hund, F. Zur Frage der chemischen Bindung. Z. Physik 73, I (1931).

51. Hund, F. Zur Frage der chemischen Bindung. 11. Zum Verständis der organischen Chemie. Z. Physik 73, 565 (1931).

52. Mulliken, R. S. The assignment of quantum numbers for electrons in molecules. 1. Phys. Rev. 32, 186 (1928).

53. Mulliken, R. S. The assignment of quantum numbers for electrons in molecules. II. Correlation of molecular and atomic states. Phys. Rev. 32, 761 (1928).

54. Löwdin, P. 0. and Pullman, B., eds. Molecular orbital theory in chemistry, physics, and biology. New York, N.Y., Academic Press. 1964.

55. Hückel, E. Zur Quantentheorie der Doppelbindung. Z. Physik 60, 423 (1930).

56. Platt, J. R. Systematics of the electronic spectra of conjugated molecules: a source book. New York, N.Y., John Wi ley \& Sons, Inc. 1964.

57. Platt, J.R. and Ruedenberg, K. Free-electron theory of conjugated molecules: a source book. New York, N.Y., John Wiley $\varepsilon$ Sons, Inc. 1964.

58. McGlynn, S. P., Vanquickenborne, L. G., Kinoshita, M., and Carroll, D. G. Introduction to applied quantum chemistry. New York, N.Y., Holt, Rinehart and Winston, Inc. 1972. 
59. Salem, L. The molecular orbital theory of conjugated systems. New York, N.Y., W. A. Benjamin, Inc. 1966.

60. Gordon, M. S. A molecular orbital theory of internal rotation. J. Am. Chem. Soc. 91, 3122 (1969).

61. Ruedenberg, K. The physical nature of the chemical bond. Rev. Mod. Phys. 34, 326 (1962).

62. Slater, J. C. Quantum theory of atomic structure. Vols. I and 11 . New York; N.Y., McGraw-Hill Book Co., Inc. 1960.

63. England, W. and Gordon, M. S. On energy localization of approximate molecular orbitals. J. Am. Chem. Soc. 9l, 6864 (1969).

64. Mulliken, R. S. Electronic population analysis on LCAO-MO molecular wave functions. 1. J. Chem. Phys. 23, 1833 (1955).

65. Muliliken, R. S. Electronic population analys is on LCAO-MO molecular wave functions. 11. Overlap populations, bond orders, and covalent bond energies. J. Chem. Phys. 23, 1841 (1955).

66. Pople, J. A. and Gordon, M. S. Molecular orbitai theory of the electronic structure of organic compounds. 1. Substituent effects and dipole moments. J. Am. Chem. Soc. 89,4253 (1967).

67. Meyer, H. and Schweig, A. An application of the CNDO/2 and INDO. theories to the calculation of molecular quadrupole moments. Chem. Phys. Lett. 9, 451 (1971).

68. Beveridge, D. L. and MClver, J.W. INDO molecular orbital study of hyperfine tensors: theory, methodology, and applications to $\mathrm{CH}$, $\mathrm{CH}_{3}$, and radacaloid derivatives of malonic acid. J. Chem. Phys. 54, .4681 (1971).

69. Gordon, M. S. and England, W. Localized charge distributions. IV. The internal rotation barrier in borazane. Chem. Phys. Lett., in the press, ca. 1972.

70. Gordon, M. S. and England, W. Localized charge distributions: V. The internal rotation barriers in methylamine, methyl alcohol, propene, and acetaldehyde. J. Am. Chem. Soc., in the press, ca. 1972.

71. Gordon, M. S. and Pople, J. A. Approximate self-consistent molecular orbital theory. VI. INDO calculated equilibrium geometries. J. Chem. Phys. 49, 4643 (1968).

72. Gordon, M. S. and Fischer, H. A molecular orbital study of the isomerization mechanism of diazacumulenes. J. Am. Chem. Soc. 90, 2471 (1968). 
73. Fischer, H. and Kollmar, H. Energy partioning with the CNDO method. Theor. Chim. Acta 16, 163 (1970).

74. Purcell, K. F. and Collins, J. M. Semiempirical SCF-LCAO-MO calculations of carbonyl donor basicities and carbonyl adduct structures. Protonated formaldehyde and acetaldehyde. J. Am. Chem. Soc. $92,465(1970)$.

75. Pullman, A. and Berthod, H. On the electronic structure of the hydrogen bond: formamide and its dimers. Theor. Chim. Acta 10, 461 (1968).

76. Del Bene, J. and Jaffé, H. H. Use of the CNDO method in spectroscopy. I. Benzene, pyridine, and the diazines. J. Chem. Phys. 48, 1807 (1968).

77. Pauling, L. The nature of the chemical bond. Application of results obtained from the quantum mechanics and from a theory of paramagnetic susceptibility to the structure of molecules. J. Am. Chem. Soc. 53, 1367 (1931).

78. Slater, J. C. Directed valence in polyatomic molecules. Phys. Rev. 37, 481 (1931).

79. Morrison, R. T. and Boyd, R. N. Organic chemistry. 2nd ed. Boston, Mass., Allyn and Bacon, Inc. 1966.

80. Coulson, C. A. Valence. 2nd ed. London, Oxford University Press. 1961.

81. Lennard-Jones, J. E. and Pople, J. A. The molecular orbital theory of chemical valency. IV. The significance of equivalent orbitals. Proc. Roy. Soc. (London) Al98, 166 (1950).

82. Hamermesh, M. Group theory. Reading, Mass., Addison-Wesley Publishing Co., inc. 1962.

83. Kuo, S. S. Numerical methods and computers. Reading, Mass., Addison-Wesley Publishing Co., Inc. 1965.

84. England, $W$. Continuous degeneracy and energy localization of molecular orbitals. Int. Journ. Quant. Chem. $v, 683$ (1971).

85. Mulliken, R. S. Quelques aspects de la théorie des orbitales moléculaires. J. Chim. Phys. 46,497 (1949).

86. Trindle, C. and Sinanoglu, 0 . Semi-empirical method for the determination of localized orbitals in molecules. J. Chem. Phys: 49, 65 (1968). 
87. Pople, J. A. and Santry, D. P. A molecular orbital theory of hydrocarbons 1. Bond delocalization in paraffins. Mol. Phys. 7, 269 (1963).

88. Pople, J. A. and Santry, D. P. A molecular orbital theory of hydrocarbons 11. Ethane, ethylene and acetylene. Mol. Phys. 9, 301 (1965).

89. Pople, J. A. and Santry, D. P. A molecular orbital theory of hydrocarbons 11l. Nuclear spin coupling constants. Mol. Phys. 2,311

(1965).

90. Coulson, C. A. and Longuet-Higgins, H. C. The electronic structure of conjugated systems 1. General theory. Proc. Roy. Soc. (London) A191, 39 (1947).

91. Coulson, C. A. and Longuet-Higgins, H. C. The electronic structure of conjugated systems 11 . Unsaturated hydrocarbons and their heteroderivatives. Proc. Roy. Soc. (London) Al92, 16 (1947).

92. Coulson, C. A. and Longuet-Higgins, H. C. The electronic structure of conjugated systems. Parts $I I I$ and IV. III. Bond orders in unsaturated molecules. IV. Force constants and interaction constants in unsaturated hydrocarbons. Proc. Roy. Soc. (London) A193, 447 (1948).

93. Coulson, C. A. and Longuet-Higgins, H. C. The electronic structure of conjugated systems $V$. The interaction of two conjugated systems. Proc. Roy. Soc. (London) Al95, 188 (1948).

94. Gilbert, T. L. Self-consistent equatlons for localized orbitals, in polyatomic systems. In Löwdin, P. O. and Pullman, B., eds. Molecular orbitals in chemistry, physics, and biology. New York, N.Y., Academic Press. 1964.

95. Day, M. C. and Selbin, J. Theoreticai inorganic chemistry. New York, N.Y., Reinhold Publishing Corp. 1962.

96. Daniels, F. and Alberty, R. A. Physical chemistry. 3rd ed. New York, N.Y., John Wiley \& Sons, Inc. 1966.

97. Moore, W. J. Physical chemistry. 3rd ed. Englewood Cliffs, N.Y., Prentice-Hall, Inc. 1962.

98. Glasstone, S. Textbook of physical chemistry. 2nd ed. New York, N.Y., D. van Nostrand Co., Inc. 1946.

99. Barrow, G. M. Physical Chemistry. New York, N.Y., McGraw-Hill Book Co., Inc. 1961. 
100. Gray, H. B. Electrons and chemical bonding. New York, N.Y., W. A. Benjamin, Inc. 1964.

101. Brand, J. C. D. and Speakman, J. C. Molecular structure. London, Edward Arnold (Publishers) Ltd. 1960.

102. Ramsey, N. F. Electron coupled interactions between nuclear spins in molecules. Phys. Rev. 91, 303 (1953).

103. McConnell, H. M. Molecular orbital approximation to electron coupled interaction between nuclear spins. J. Chem. Phys. 24, 460 (1956).

104. Pople, J. A. and Santry, D. P. Molecular orbital theory of nuclear spin coupling constants. Mol. Phys. 8, I (1964).

105. Memory, J. D. Quantum theory of magnetic resonance parameters. New York, N.Y., McGraw-Hill Book Co. 1968.

106. Sanderson, R. T. Chemical bonds and bond energy. New York, N.Y., Academic Press. 1971.

107. Jackson, J. D. Classical Electrodynamics. New York, N.Y., John Wiley \& Sons, Inc. 1962.

108. Sakurai, J. J. Advanced quantum mechanics. Reading, Mass., Addison-Wesley Publishing Co.,. Inc. 1967.

109. Weidner, R. T. and Sells, R. L. Elementary modern physics: Boșton, Mass., Allyn and Bacon, Inc. 1960.

110. Emsley, J. W., Feeney, J., and Sutcliffe, L. H. High resolution nuclear magnetic resonance spectroscopy. Vol. 2. New York, N.Y., Pergamon Press. 1966.

11l. Pauncz, R. Alternant molecular orbital method. Philadelphia, Pa., W. B. Saunders Co. 1967.

112. Gould, E. S. Mechanism and structure in organic chemistry. New York, N.Y., Holt, Rinehart and Winston. 1959.

113. Mulliken, R. S., Rieke, C. A., and Brown, W. G. Hyperconjugation. J. Am. Chem. Soc. 63,41 (1941).

114. Mulliken, R. S. Intensities of electronic transitions in molecular spectra. IV. Cyclic dienes and hyperconjugation. J. Chem. Phys. 7. 339 (1939).

115. Dewar, M. J. S. Hyperconjugation. New York, N.Y., Ronald Press. 1962. 
116. Eisenbarger, P. and Marra, W. C. I dentification of localized bonds in the hydrocarbons. Phys. Rev. Lett. 27, 1413.(1971).

117. Kilby, G. E. A wave-mechanical derivation of the intensity distribution of the Compton Iine. Proc. Phys. Soc. (London) 86, 1037 (1965).

118. Eps tein, 1. R. and Lipscomb, W. N. Molecular momentum distributions and Compton profiles. 1. General theory and boron hydrides. J. Chem. Phys. 53, 4418 (1970).

119. Weiss, R. J. X-ray determination of electron distributions. New York, N.Y.,. John Wi ley \& Sons, Inc. 1966.

120. Kaplan, W. Advanced calculus. Reading, Mass., Addison-Wesley Publishing Co., Inc. 1952.

121. Wahl, A. C., Bertoncini, P. J., Kaiser, K., and Land, R. BISON a FORTRAN computer system for the calculation of analytic selfconsistent-field wavefunctions, properties, and charge densities for diatomic molecules. Argonne National Laboratory Technical Report ANL-7271 and AEC Research and Development Report. TID-4500 (Argonne, Iil.). 1968.

122. Hartree, D. R. The calculation of atomic structures. New York, N.Y., John Wiley \& Sons, Inc. 1957.

123. Artmann, K. Zur Quantentheorie der gewinkelten Vạlenz, 1. Mitteilung: Eigenfunktion und Valenzbetatigung des Zentralatoms. Zeits. f. Naturfor. 1, 426 (1946).

124. Zimmerman; H. K. and Van Rysselberghe, P. Directed valence as a property of determinant wave functions. J. Chem. Phys. 17, 598 (1949).

125. Linnett, J. W. and Poë, A. J. Directed valency in elements of the first short period.' Trans. Faraday Soc. 47, 1033 (1951). 


\section{ACKNOWLEDGEMENTS}

Dr: James W. Richardson, Purdue University, who first introduced me to theoretical chemistry.

Or. Mark S. Gordon, North Dakota State University, who first introduced me to semiempirical LCAO-MO-SCF theory, and whose enthusiasm is contagious.

Dr. Klaus Ruedenberg, major professor, whose profundity, technical competence, and humanity did much to make graduate school worthwhile. 